Network Working Group

Request for Comments: 3530

Obsoletes: 3010

Category: Standards Track
S. Shepler

B. Callaghan

D. Robinson

R. Thurlow

Sun Microsystems, Inc.

C. Beame

Hummingbird Ltd.

M. Eisler

D. Noveck

Network Appliance, Inc.

April 2003

Network File System (NFS) version 4 Protocol

Status of this Memo

This document specifies an Internet standards track protocol for the Internet community, and requests discussion and suggestions for improvements. Please refer to the current edition of the "Internet Official Protocol Standards" (STD 1) for the standardization state and status of this protocol. Distribution of this memo is unlimited.

Copyright Notice

Copyright (C) The Internet Society (2003). All Rights Reserved.

Abstract

The Network File System (NFS) version 4 is a distributed filesystem protocol which owes heritage to NFS protocol version 2, RFC 1094, and version 3, RFC 1813. Unlike earlier versions, the NFS version 4 protocol supports traditional file access while integrating support for file locking and the mount protocol. In addition, support for strong security (and its negotiation), compound operations, client caching, and internationalization have been added. Of course, attention has been applied to making NFS version 4 operate well in an Internet environment.

This document replaces RFC 3010 as the definition of the NFS version 4 protocol.

Key Words

The key words "MUST", "MUST NOT", "REQUIRED", "SHALL", "SHALL NOT", "SHOULD", "SHOULD NOT", "RECOMMENDED", "MAY", and "OPTIONAL" in this document are to be interpreted as described in [RFC2119]. 
Table of Contents

1. Introduction . . . . . . . . . . . . . . . . . . . . . . . 8

1.1. Changes since RFC 3010 . . . . . . . . . . . . . 8

1.2. NFS version 4 Goals. . . . . . . . . . . . . . . 9

1.3. Inconsistencies of this Document with Section 18 . . 9

1.4. Overview of NFS version 4 features . . . . . . . . 10

1.4.1. RPC and Security . . . . . . . . . . . . . 10

1.4.2. Procedure and Operation Structure. . . . . . 10

1.4.3. Filesystem Mode. . . . . . . . . . . . 11

1.4.3.1. Filehandle Types . . . . . . . 11

1.4.3.2. Attribute Types. . . . . . . . 12

1.4.3.3. Filesystem Replication and

Migration. . . . . . . . . 13

1.4.4. OPEN and CLOSE . . . . . . . . . . . . . . . 13

1.4.5. File locking . . . . . . . . . . . . . 13

1.4.6. Client Caching and Delegation. . . . . . . 13

1.5. General Definitions. . . . . . . . . . . . . 14

2. Protocol Data Types. . . . . . . . . . . . . . . 16

2.1. Basic Data Types . . . . . . . . . . . . . 16

2.2. Structured Data Types. • . . . . . . . . . • . . 18

3. RPC and Security Flavor. . . . . . . . . . . . . . . . 23

3.1. Ports and Transports . . . . . . . . . . . . 23

3.1.1. Client Retransmission Behavior . . . . . . 24

3.2. Security Flavors . . . . . . . . . . . . . . 25

3.2.1. Security mechanisms for NFS version 4. . . 25

3.2.1.1. Kerberos V5 as a security triple. 25

3.2.1.2. LIPKEY as a security triple. . . 26

3.2.1.3. SPKM-3 as a security triple. . . 27

3.3. Security Negotiation . . . . . . . . . . . . 27

3.3.1. SECINFO. . . . . . . . . . . . . . 28

3.3.2. Security Error................ . 28

3.4. Callback RPC Authentication. . . . . . . . . . . 28

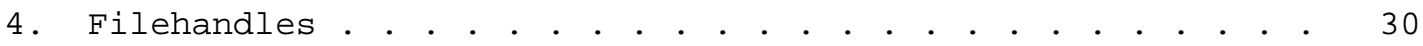

4.1. Obtaining the First Filehandle . . . . . . . . . . 30

4.1.1. Root Filehandle. . . . . . . . . . . . . 31

4.1.2. Public Filehandle. . . . . . . . . . . . 31

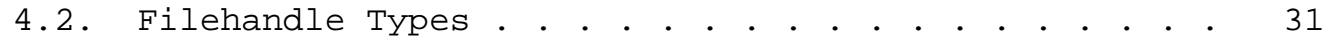

4.2.1. General Properties of a Filehandle . . . . . 32

4.2.2. Persistent Filehandle. . . . . . . . . . . 32

4.2.3. Volatile Filehandle. . . . . . . . . . . 33

4.2.4. One Method of Constructing a

Volatile Filehandle. . . . . . . . . . 34

4.3. Client Recovery from Filehandle Expiration . . . . . 35

5. File Attributes. . . . . . . . . . . . . . . . . . 35

5.1. Mandatory Attributes . . . . . . . . . . . . . 37

5.2. Recommended Attributes . . . . . . . . . . . 37

5.3. Named Attributes . . . . . . . . . . . . 37 
5.4. Classification of Attributes . . . . . . . . . . . 38

5.5. Mandatory Attributes - Definitions . . . . . . . . . 39

5.6. Recommended Attributes - Definitions . . . . . . . . 41

5.7. Time Access. . . . . . . . . . . . . . . . . . 46

5.8. Interpreting owner and owner_group . . . . . . . . 47

5.9. Character Case Attributes. . . . . . . . . . . . 49

5.10. Quota Attributes . . . . . . . . . . . . . . . 49

5.11. Access Control Lists . . . . . . . . . . . . . 50

5.11.1. ACE type... . . . . . . . . . . . 51

5.11.2. ACE ACCess Mask. . . . . . . . . . 52

5.11.3. ACE flag. . . . . . . . . . . . . 54

5.11.4. $\mathrm{ACE}$ who . . . . . . . . . . . . . 55

5.11.5. Mode Attribute . . . . . . . . . . 56

5.11.6. Mode and ACL Attribute. . . . . . . 57

5.11.7. mounted_on_fileid. . . . . . . . . 57

6. Filesystem Migration and Replication . . . . . . . . . 58

6.1. Replication. • . • . . . . . . . . . . . . 58

6.2. Migration. . . . . . . . . . . . . . . . 59

6.3. Interpretation of the fs_locations Attribute. . . . 60

6.4. Filehandle Recovery for Migration or Replication . . 61

7. NFS Server Name Space . . . . . . . . . . . . . . . . . 61

7.1. Server Exports . . . . . . . . . . . . . . . 61

7.2. Browsing Exports . . . . . . . . . . . . . 62

7.3. Server Pseudo Filesystem . . . . . . . . . . . . 62

7.4. Multiple Roots . . . . . . . . . . . . . . 63

7.5. Filehandle Volatility. . . . . . . . . . . . 63

7.6. Exported Root. . . . . . . . . . . . . 63

7.7. Mount Point Crossing . . . . . . . . . . . . 63

7.8. Security Policy and Name space Presentation. . . . . 64

8. File Locking and Share Reservations. . . . . . . . . . . 65

8.1. Locking. . . . . . . . . . . . . . . . 65

8.1.1. Client ID. . . . . . . . . . . . . . 66

8.1.2. Server Release of Clientid . . . . . . . 69

8.1.3. lock_owner and stateid Definition. . . . 69

8.1.4. Use of the stateid and Locking . . . . . . 71

8.1.5. Sequencing of Lock Requests. . . . . . . 73

8.1.6. Recovery from Replayed Requests. . . . . 74

8.1.7. Releasing lock_owner State . . . . . . . 74

8.1.8. Use of Open Confirmation . . . . . . . . 75

8.2. Lock Ranges. . . . . . . . . . . . . . . . . 76

8.3. Upgrading and Downgrading Locks. . . . . . . . . 76

8.4. Blocking Locks . . . . . . . . . . . . . . . 77

8.5. Lease Renewal. . . . . . . . . . . . . . . 77

8.6. Crash Recovery . . . . . . . . . . . . . . . 78

8.6.1. Client Failure and Recovery. . . . . . . 79

8.6.2. Server Failure and Recovery. . . . . . . 79

8.6.3. Network Partitions and Recovery. . . . . . 81

8.7. Recovery from a Lock Request Timeout or Abort. . . 85 
8.8. Server Revocation of Locks. . . . . . . . . . . 85

8.9. Share Reservations. . . . . . . . . . . . . 86

8.10. OPEN/CLOSE Operations . . . . . . . . . . . . 87

8.10.1. Close and Retention of State

Information. . . . . . . . . . . . 88

8.11. Open Upgrade and Downgrade. . . . . . . . . . . . 88

8.12. Short and Long Leases. . . . . . . . . . . . . . 89

8.13. Clocks, Propagation Delay, and Calculating Lease

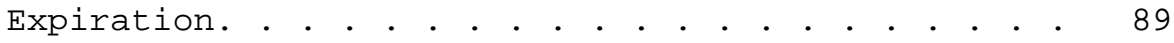

8.14. Migration, Replication and State. . . . . . . . 90

8.14.1. Migration and State. . . . . . . . . 90

8.14.2. Replication and State. . . . . . . . . 91

8.14.3. Notification of Migrated Lease . . . . . 92

8.14.4. Migration and the Lease_time Attribute. . 92

9. Client-Side Caching . . . . . . . . . . . . . . . . 93

9.1. Performance Challenges for Client-Side Caching. . . 93

9.2. Delegation and Callbacks. . . . . . . . . . . . . 94

9.2.1. Delegation Recovery . . . . . . . . . . . 96

9.3. Data Caching. . . . . . . . . . . . . . 98

9.3.1. Data Caching and OPENs . . . . . . . . 98

9.3.2. Data Caching and File Locking. . . . . . . 99

9.3.3. Data Caching and Mandatory File Locking. . 101

9.3.4. Data Caching and File Identity . . . . . 101

9.4. Open Delegation . . . . . . . . . . . . . . 102

9.4.1. Open Delegation and Data Caching . . . . 104

9.4.2. Open Delegation and File Locks . . . . . 106

9.4.3. Handling of CB_GETATTR . . . . . . . . 106

9.4.4. Recall of Open Delegation. . . . . . . 109

9.4.5. Clients that Fail to Honor

Delegation Recalls . • . . • • • . . 111

9.4.6. Delegation Revocation. . . . . . . . . 112

9.5. Data Caching and Revocation . . . . . . . . . 112

9.5.1. Revocation Recovery for Write Open

Delegation . • • • • . . . . . . 113

9.6. Attribute Caching . . . . . . . . . . . . . . . 113

9.7. Data and Metadata Caching and Memory Mapped Files . 115

9.8. Name Caching . . . . . . . . . . . . . 118

9.9. Directory Caching • . . . . . . . . . . . . . . . . 119

10. Minor Versioning . . . . . . . . . . . . . . . . . 120

11. Internationalization . . . . . . . . . . . . . . . . 122

11.1. Stringprep profile for the utf8str_cs type. . . . 123

11.1.1. Intended applicability of the

nfs4_cs_prep profile. • . . . . . . . 123

11.1.2. Character repertoire of nfs4_cs_prep. . . 124

11.1.3. Mapping used by nfs4_cs_prep . . . . . . 124

11.1.4. Normalization used by nfs4_cs_prep . . . 124

11.1.5. Prohibited output for nfs4_cs_prep . . . 125

11.1.6. Bidirectional output for nfs4_cs_prep. . . 125 
11.2. Stringprep profile for the utf8str_cis type . . . . 125

11.2.1. Intended applicability of the

nfs4_cis_prep profile. . . . . . . . . 125

11.2.2. Character repertoire of nfs4_cis_prep . . 125

11.2.3. Mapping used by nfs4_cis_prep . . . . . 125

11.2.4. Normalization used by nfs4_cis_prep . . . 125

11.2.5. Prohibited output for nfs4_cis_prep . . . 126

11.2.6. Bidirectional output for nfs4_cis_prep . . 126

11.3. Stringprep profile for the utf8str_mixed type . . . 126

11.3.1. Intended applicability of the

nfs4_mixed_prep profile. . . . . . . 126

11.3.2. Character repertoire of nfs4_mixed_prep . 126

11.3.3. Mapping used by nfs4_cis_prep . . . . . 126

11.3.4. Normalization used by nfs4_mixed_prep . . 127

11.3.5. Prohibited output for nfs4_mixed_prep . . 127

11.3.6. Bidirectional output for nfs4_mixed_prep . 127

11.4. UTF-8 Related Errors. . . . . . . . . . . . . 127

12. Error Definitions . . . . . . . . . . . . . . . . 128

13. NFS version 4 Requests . . . . . . . . . . . . . . . 134

13.1. Compound Procedure. . . . . . . . . . . . . . 134

13.2. Evaluation of a Compound Request. . . . . . . . . 135

13.3. Synchronous Modifying Operations. . . . . . . . . 136

13.4. Operation Values. . . . . . . . . . . . . 136

14. NFS version 4 Procedures . . . . . . . . . . . . . . . . 136

14.1. Procedure 0: NULL - No Operation. . . . . . . . . 136

14.2. Procedure 1: COMPOUND - Compound Operations . . . 137

14.2.1. Operation 3: ACCESS - Check AcCess

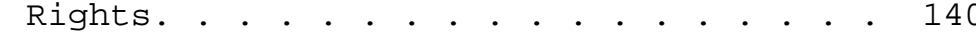

14.2.3. Operation 5: COMMIT - Commit

Cached Data.............. . 144

14.2.4. Operation 6: CREATE - Create a

Non-Regular File Object . . . . . . . 147

14.2.5. Operation 7: DELEGPURGE -

Purge Delegations Awaiting Recovery . . 150

14.2.6. Operation 8: DELEGRETURN - Return

Delegation. . . . . • • • • . . . . 151

14.2.7. Operation 9: GETATTR - Get Attributes . . 152

14.2.8. Operation 10: GETFH - Get Current

Filehandle. . . . . . . . . . . . 153

14.2.9. Operation 11: LINK - Create Link to a

File. . . . . . . . . . . . . . . 154

14.2.10. Operation 12: LOCK - Create Lock . . . 156

14.2.11. Operation 13: LOCKT - Test For Lock . . 160

14.2.12. Operation 14: LOCKU - Unlock File • . . 162

14.2.13. Operation 15: LOOKUP - Lookup Filename. . 163

14.2.14. Operation 16: LOOKUPP - Lookup

Parent Directory. . . . . . . . . 165 


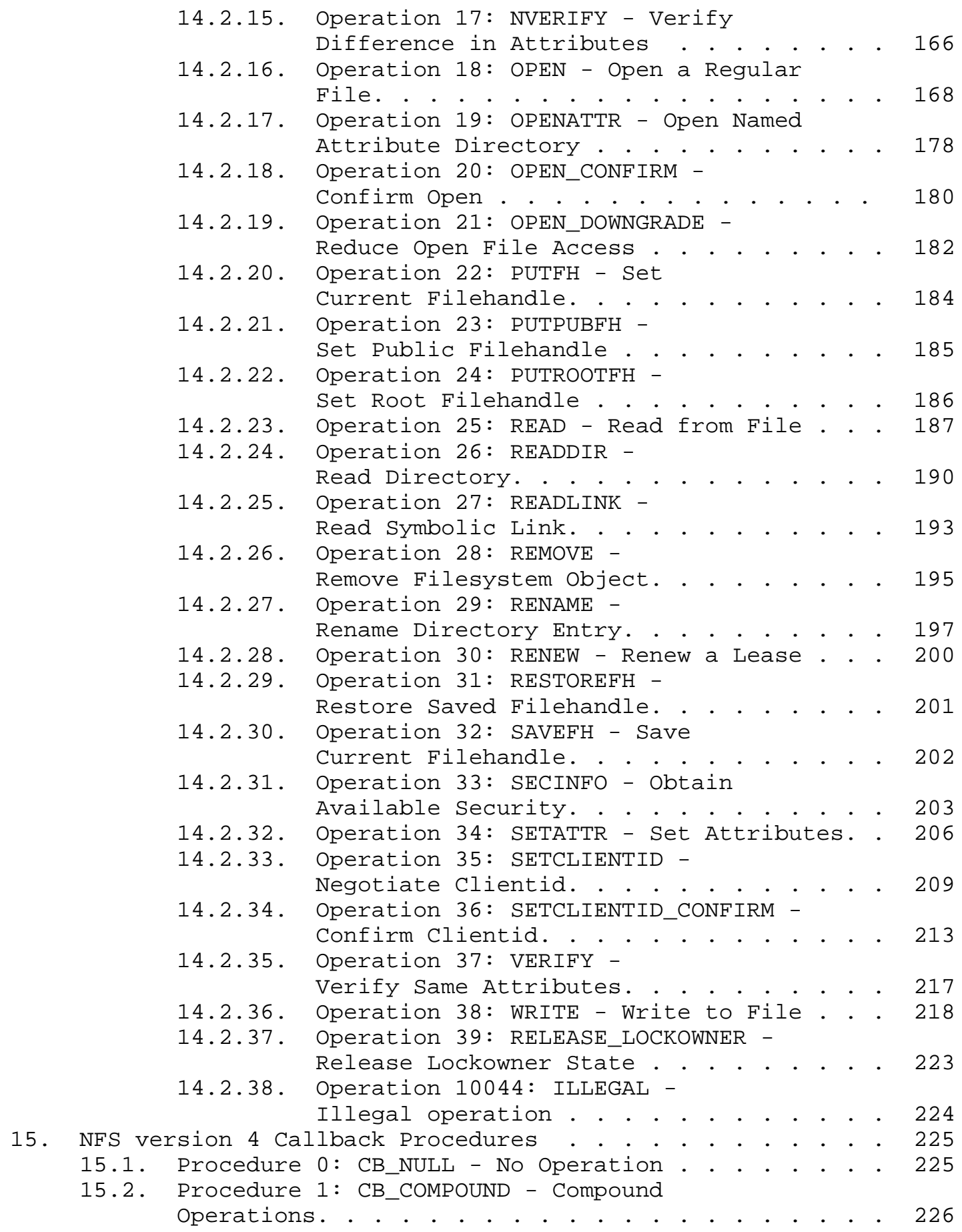




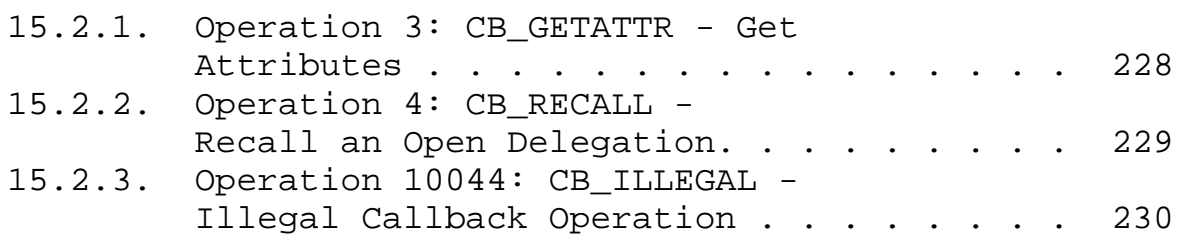

23. Full Copyright Statement . . . . . . . . . . . . . . 275 


\section{Introduction}

\subsection{Changes since RFC 3010}

This definition of the NFS version 4 protocol replaces or obsoletes the definition present in [RFC3010]. While portions of the two documents have remained the same, there have been substantive changes in others. The changes made between [RFC3010] and this document represent implementation experience and further review of the protocol. While some modifications were made for ease of implementation or clarification, most updates represent errors or situations where the [RFC3010] definition were untenable.

The following list is not all inclusive of all changes but presents some of the most notable changes or additions made:

- The state model has added an open_owner4 identifier. This was done to accommodate Posix based clients and the model they use for file locking. For Posix clients, an open_owner4 would correspond to a file descriptor potentially shared amongst a set of processes and the lock_owner4 identifier would correspond to a process that is locking a file.

- Clarifications and error conditions were added for the handling of the owner and group attributes. Since these attributes are string based (as opposed to the numeric uid/gid of previous versions of NFS), translations may not be available and hence the changes made.

- Clarifications for the ACL and mode attributes to address evaluation and partial support.

- For identifiers that are defined as XDR opaque, limits were set on their size.

- Added the mounted_on_filed attribute to allow Posix clients to correctly construct local mounts.

- Modified the SETCLIENTID/SETCLIENTID_CONFIRM operations to deal correctly with confirmation details along with adding the ability to specify new client callback information. Also added clarification of the callback information itself.

- Added a new operation LOCKOWNER_RELEASE to enable notifying the server that a lock_owner4 will no longer be used by the client.

- RENEW operation changes to identify the client correctly and allow for additional error returns. 
- Verify error return possibilities for all operations.

- Remove use of the pathname4 data type from LOOKUP and OPEN in favor of having the client construct a sequence of LOOKUP operations to achieive the same effect.

- Clarification of the internationalization issues and adoption of the new stringprep profile framework.

1.2. NFS Version 4 Goals

The NFS version 4 protocol is a further revision of the NFS protocol defined already by versions 2 [RFC1094] and 3 [RFC1813]. It retains the essential characteristics of previous versions: design for easy recovery, independent of transport protocols, operating systems and filesystems, simplicity, and good performance. The NFS version 4 revision has the following goals:

- Improved access and good performance on the Internet.

The protocol is designed to transit firewalls easily, perform well where latency is high and bandwidth is low, and scale to very large numbers of clients per server.

o Strong security with negotiation built into the protocol.

The protocol builds on the work of the ONCRPC working group in supporting the RPCSEC_GSS protocol. Additionally, the NFS version 4 protocol provides a mechanism to allow clients and servers the ability to negotiate security and require clients and servers to support a minimal set of security schemes.

o Good cross-platform interoperability.

The protocol features a filesystem model that provides a useful, common set of features that does not unduly favor one filesystem or operating system over another.

- Designed for protocol extensions.

The protocol is designed to accept standard extensions that do not compromise backward compatibility.

1.3. Inconsistencies of this Document with Section 18

Section 18, RPC Definition File, contains the definitions in XDR description language of the constructs used by the protocol. Prior to section 18, several of the constructs are reproduced for purposes 
of explanation. The reader is warned of the possibility of errors in the reproduced constructs outside of Section 18. For any part of the document that is inconsistent with section 18, section 18 is to be considered authoritative.

\subsection{Overview of NFS version 4 Features}

To provide a reasonable context for the reader, the major features of NFS version 4 protocol will be reviewed in brief. This will be done to provide an appropriate context for both the reader who is familiar with the previous versions of the NFS protocol and the reader that is new to the NFS protocols. For the reader new to the NFS protocols, there is still a fundamental knowledge that is expected. The reader should be familiar with the XDR and RPC protocols as described in [RFC1831] and [RFC1832]. A basic knowledge of filesystems and distributed filesystems is expected as well.

\subsubsection{RPC and Security}

As with previous versions of NFS, the External Data Representation (XDR) and Remote Procedure Call (RPC) mechanisms used for the NFS version 4 protocol are those defined in [RFC1831] and [RFC1832]. To meet end to end security requirements, the RPCSEC_GSS framework [RFC2203] will be used to extend the basic RPC security. With the use of RPCSEC_GSS, various mechanisms can be provided to offer authentication, integrity, and privacy to the NFS version 4 protocol. Kerberos V5 will be used as described in [RFC1964] to provide one security framework. The LIPKEY GSS-API mechanism described in [RFC2847] will be used to provide for the use of user password and server public key by the NFS version 4 protocol. With the use of RPCSEC_GSS, other mechanisms may also be specified and used for NFS version 4 security.

To enable in-band security negotiation, the NFS version 4 protocol has added a new operation which provides the client a method of querying the server about its policies regarding which security mechanisms must be used for access to the server's filesystem resources. With this, the client can securely match the security mechanism that meets the policies specified at both the client and server.

\subsubsection{Procedure and Operation Structure}

A significant departure from the previous versions of the NFS protocol is the introduction of the COMPOUND procedure. For the NFS version 4 protocol, there are two RPC procedures, NULL and COMPOUND. The COMPOUND procedure is defined in terms of operations and these operations correspond more closely to the traditional NFS procedures. 
With the use of the COMPOUND procedure, the client is able to build simple or complex requests. These COMPOUND requests allow for a reduction in the number of RPCs needed for logical filesystem operations. For example, without previous contact with a server a client will be able to read data from a file in one request by combining LOOKUP, OPEN, and READ operations in a single COMPOUND RPC. With previous versions of the NFS protocol, this type of single request was not possible.

The model used for COMPOUND is very simple. There is no logical OR or ANDing of operations. The operations combined within a COMPOUND request are evaluated in order by the server. Once an operation returns a failing result, the evaluation ends and the results of all evaluated operations are returned to the client.

The NFS version 4 protocol continues to have the client refer to a file or directory at the server by a "filehandle". The COMPOUND procedure has a method of passing a filehandle from one operation to another within the sequence of operations. There is a concept of a "current filehandle" and "saved filehandle". Most operations use the "current filehandle" as the filesystem object to operate upon. The "saved filehandle" is used as temporary filehandle storage within a COMPOUND procedure as well as an additional operand for certain operations.

\subsubsection{Filesystem Model}

The general filesystem model used for the NFS version 4 protocol is the same as previous versions. The server filesystem is hierarchical with the regular files contained within being treated as opaque byte streams. In a slight departure, file and directory names are encoded with UTF-8 to deal with the basics of internationalization.

The NFS version 4 protocol does not require a separate protocol to provide for the initial mapping between path name and filehandle. Instead of using the older MOUNT protocol for this mapping, the server provides a ROOT filehandle that represents the logical root or top of the filesystem tree provided by the server. The server provides multiple filesystems by gluing them together with pseudo filesystems. These pseudo filesystems provide for potential gaps in the path names between real filesystems.

\subsubsection{Filehandle Types}

In previous versions of the NFS protocol, the filehandle provided by the server was guaranteed to be valid or persistent for the lifetime of the filesystem object to which it referred. For some server implementations, this persistence requirement has been difficult to 
meet. For the NFS version 4 protocol, this requirement has been relaxed by introducing another type of filehandle, volatile. With persistent and volatile filehandle types, the server implementation can match the abilities of the filesystem at the server along with the operating environment. The client will have knowledge of the type of filehandle being provided by the server and can be prepared to deal with the semantics of each.

\subsubsection{Attribute Types}

The NFS version 4 protocol introduces three classes of filesystem or file attributes. Like the additional filehandle type, the classification of file attributes has been done to ease server implementations along with extending the overall functionality of the NFS protocol. This attribute model is structured to be extensible such that new attributes can be introduced in minor revisions of the protocol without requiring significant rework.

The three classifications are: mandatory, recommended and named attributes. This is a significant departure from the previous attribute model used in the NFS protocol. Previously, the attributes for the filesystem and file objects were a fixed set of mainly UNIX attributes. If the server or client did not support a particular attribute, it would have to simulate the attribute the best it could.

Mandatory attributes are the minimal set of file or filesystem attributes that must be provided by the server and must be properly represented by the server. Recommended attributes represent different filesystem types and operating environments. The recommended attributes will allow for better interoperability and the inclusion of more operating environments. The mandatory and recommended attribute sets are traditional file or filesystem attributes. The third type of attribute is the named attribute. A named attribute is an opaque byte stream that is associated with a directory or file and referred to by a string name. Named attributes are meant to be used by client applications as a method to associate application specific data with a regular file or directory.

One significant addition to the recommended set of file attributes is the Access Control List (ACL) attribute. This attribute provides for directory and file access control beyond the model used in previous versions of the NFS protocol. The ACL definition allows for specification of user and group level access control. 


\subsubsection{Filesystem Replication and Migration}

With the use of a special file attribute, the ability to migrate or replicate server filesystems is enabled within the protocol. The filesystem locations attribute provides a method for the client to probe the server about the location of a filesystem. In the event of a migration of a filesystem, the client will receive an error when operating on the filesystem and it can then query as to the new file system location. Similar steps are used for replication, the client is able to query the server for the multiple available locations of a particular filesystem. From this information, the client can use its own policies to access the appropriate filesystem location.

\subsubsection{OPEN and CLOSE}

The NFS version 4 protocol introduces OPEN and CLOSE operations. The OPEN operation provides a single point where file lookup, creation, and share semantics can be combined. The CLOSE operation also provides for the release of state accumulated by OPEN.

\subsubsection{File locking}

With the NFS version 4 protocol, the support for byte range file locking is part of the NFS protocol. The file locking support is structured so that an RPC callback mechanism is not required. This is a departure from the previous versions of the NFS file locking protocol, Network Lock Manager (NLM). The state associated with file locks is maintained at the server under a lease-based model. The server defines a single lease period for all state held by a NFS client. If the client does not renew its lease within the defined period, all state associated with the client's lease may be released by the server. The client may renew its lease with use of the RENEW operation or implicitly by use of other operations (primarily READ).

\subsubsection{Client Caching and Delegation}

The file, attribute, and directory caching for the NFS version 4 protocol is similar to previous versions. Attributes and directory information are cached for a duration determined by the client. At the end of a predefined timeout, the client will query the server to see if the related filesystem object has been updated.

For file data, the client checks its cache validity when the file is opened. A query is sent to the server to determine if the file has been changed. Based on this information, the client determines if the data cache for the file should kept or released. Also, when the file is closed, any modified data is written to the server. 
If an application wants to serialize access to file data, file locking of the file data ranges in question should be used.

The major addition to NFS version 4 in the area of caching is the ability of the server to delegate certain responsibilities to the client. When the server grants a delegation for a file to a client, the client is guaranteed certain semantics with respect to the sharing of that file with other clients. At OPEN, the server may provide the client either a read or write delegation for the file. If the client is granted a read delegation, it is assured that no other client has the ability to write to the file for the duration of the delegation. If the client is granted a write delegation, the client is assured that no other client has read or write access to the file.

Delegations can be recalled by the server. If another client requests access to the file in such a way that the access conflicts with the granted delegation, the server is able to notify the initial client and recall the delegation. This requires that a callback path exist between the server and client. If this callback path does not exist, then delegations can not be granted. The essence of a delegation is that it allows the client to locally service operations such as OPEN, CLOSE, LOCK, LOCKU, READ, WRITE without immediate interaction with the server.

\subsection{General Definitions}

The following definitions are provided for the purpose of providing an appropriate context for the reader.

Client The "client" is the entity that accesses the NFS server's resources. The client may be an application which contains the logic to access the NFS server directly. The client may also be the traditional operating system client remote filesystem services for a set of applications.

In the case of file locking the client is the entity that maintains a set of locks on behalf of one or more applications. This client is responsible for crash or failure recovery for those locks it manages.

Note that multiple clients may share the same transport and multiple clients may exist on the same network node.

Clientid A 64-bit quantity used as a unique, short-hand reference to a client supplied Verifier and ID. The server is responsible for supplying the Clientid. 
Lease An interval of time defined by the server for which the client is irrevocably granted a lock. At the end of a lease period the lock may be revoked if the lease has not been extended. The lock must be revoked if a conflicting lock has been granted after the lease interval.

All leases granted by a server have the same fixed interval. Note that the fixed interval was chosen to alleviate the expense a server would have in maintaining state about variable length leases across server failures.

Lock The term "lock" is used to refer to both record (byterange) locks as well as share reservations unless specifically stated otherwise.

Server The "Server" is the entity responsible for coordinating client access to a set of filesystems.

Stable Storage

NFS version 4 servers must be able to recover without data loss from multiple power failures (including cascading power failures, that is, several power failures in quick succession), operating system failures, and hardware failure of components other than the storage medium itself (for example, disk, nonvolatile RAM).

Some examples of stable storage that are allowable for an NFS server include:

1. Media commit of data, that is, the modified data has been successfully written to the disk media, for example, the disk platter.

2. An immediate reply disk drive with battery-backed ondrive intermediate storage or uninterruptible power system (UPS).

3. Server commit of data with battery-backed intermediate storage and recovery software.

4. Cache commit with uninterruptible power system (UPS) and recovery software.

Stateid A 128-bit quantity returned by a server that uniquely defines the open and locking state provided by the server for a specific open or lock owner for a specific file. 
Stateids composed of all bits 0 or all bits 1 have special meaning and are reserved values.

Verifier A 64-bit quantity generated by the client that the server can use to determine if the client has restarted and lost all previous lock state.

2. Protocol Data Types

The syntax and semantics to describe the data types of the NFS version 4 protocol are defined in the XDR [RFC1832] and RPC [RFC1831] documents. The next sections build upon the XDR data types to define types and structures specific to this protocol.

2.1. Basic Data Types

\begin{tabular}{|c|c|}
\hline Data Type & Definition \\
\hline int32_t & typedef int \\
\hline uint32_t & typedef unsigned int \\
\hline int 64_t & typedef hyper \\
\hline uint64_t & typedef unsigned hyper uint64_t; \\
\hline attrlist 4 & $\begin{array}{l}\text { typedef opaque attrlist } 4<>; \\
\text { Used for file/directory attributes }\end{array}$ \\
\hline bitmap 4 & $\begin{array}{ll}\text { typedef uint32_t } & \text { bitmap } 4<>\text {; } \\
\text { Used in attribute array encoding. }\end{array}$ \\
\hline changeid4 & $\begin{array}{l}\text { typedef } \quad \text { uint64_t changeid4; } \\
\text { Used in definition of change_info }\end{array}$ \\
\hline clientid4 & $\begin{array}{l}\text { typedef uint64_t clientid4; } \\
\text { Shorthand reference to client identification }\end{array}$ \\
\hline component 4 & $\begin{array}{l}\text { typedef utf8str_cs component } 4 ; \\
\text { Represents path name components }\end{array}$ \\
\hline count 4 & $\begin{array}{l}\text { typedef uint32_t count } 4 ; \\
\text { Various count parameters (READ, WRITE, COMMIT) }\end{array}$ \\
\hline length 4 & $\begin{array}{l}\text { typedef uint } 64 \text { _t } \\
\text { Describes LOCK lengths }\end{array}$ \\
\hline
\end{tabular}




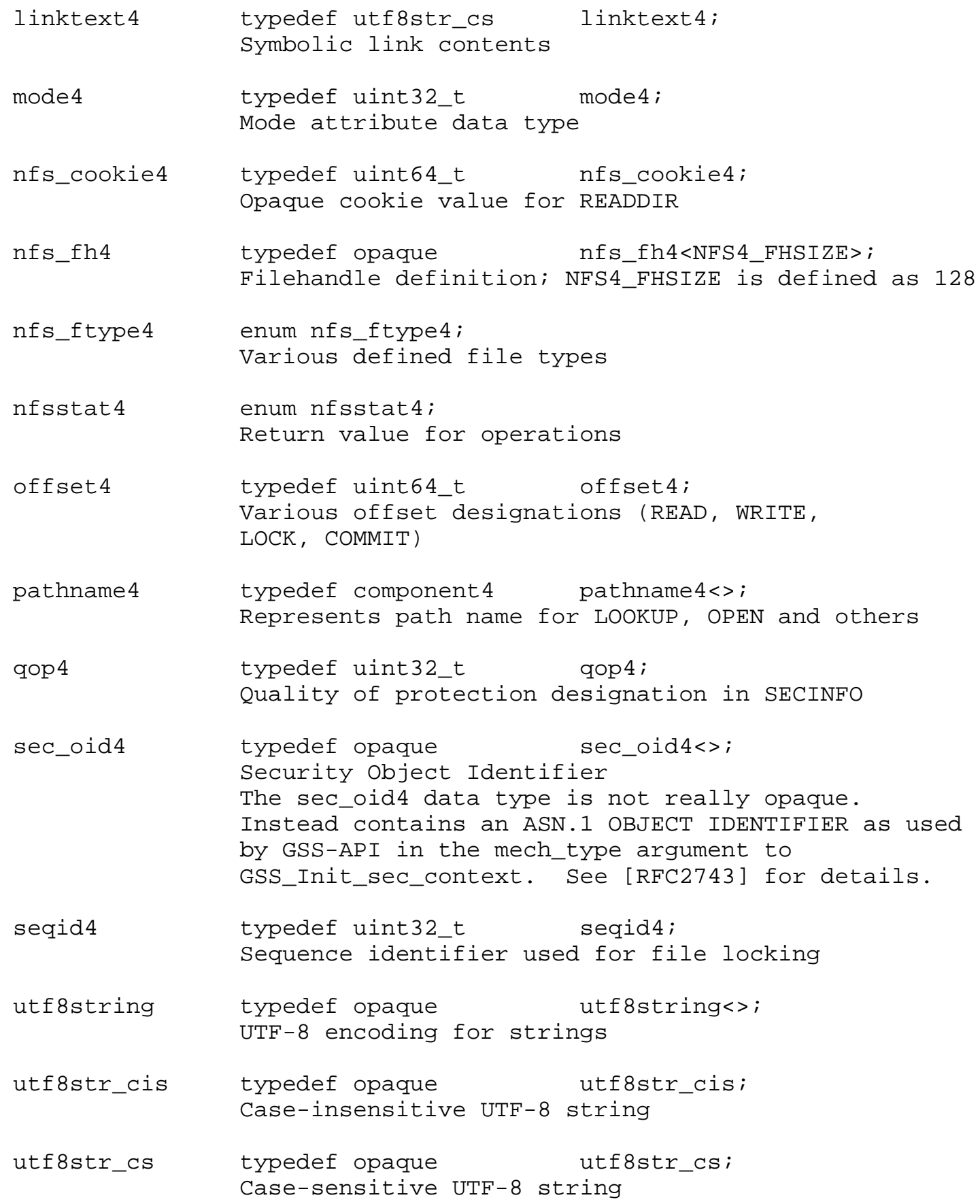


utf8str_mixed typedef opaque utf8str_mixed;

UTF-8 strings with a case sensitive prefix and a case insensitive suffix.

verifier4

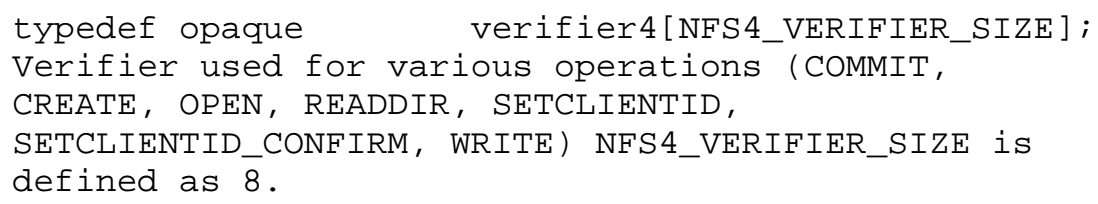

2.2. Structured Data Types

nfstime 4

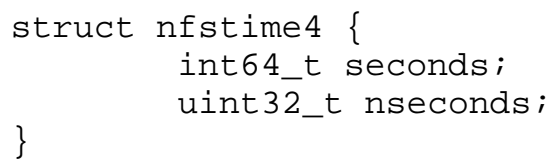

The nfstime4 structure gives the number of seconds and nanoseconds since midnight or 0 hour January 1, 1970 Coordinated Universal Time (UTC). Values greater than zero for the seconds field denote dates after the 0 hour January 1, 1970. Values less than zero for the seconds field denote dates before the 0 hour January 1, 1970. In both cases, the nseconds field is to be added to the seconds field for the final time representation. For example, if the time to be represented is one-half second before 0 hour January 1, 1970, the seconds field would have a value of negative one (-1) and the nseconds fields would have a value of one-half second (500000000). Values greater than 999,999,999 for nseconds are considered invalid.

This data type is used to pass time and date information. A server converts to and from its local representation of time when processing time values, preserving as much accuracy as possible. If the precision of timestamps stored for a filesystem object is less than defined, loss of precision can occur. An adjunct time maintenance protocol is recommended to reduce client and server time skew.

time_how 4

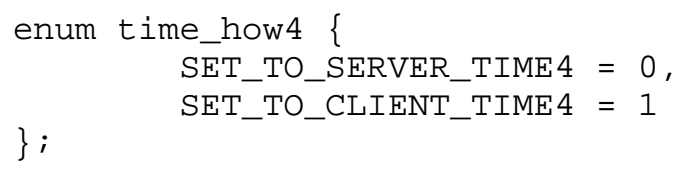


The fs_location4 and fs_locations4 data types are used for the fs_locations recommended attribute which is used for migration and replication support.

fattr 4

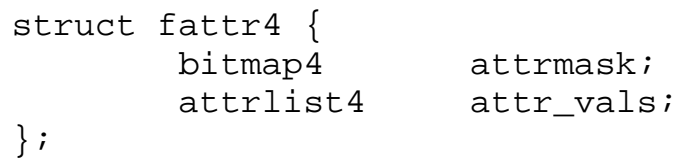

The bitmap is a counted array of 32 bit integers used to contain bit values. The position of the integer in the array that contains bit $n$ can be computed from the expression ( $\mathrm{n} / 32$ ) and its bit within that integer is $(n \bmod 32)$.

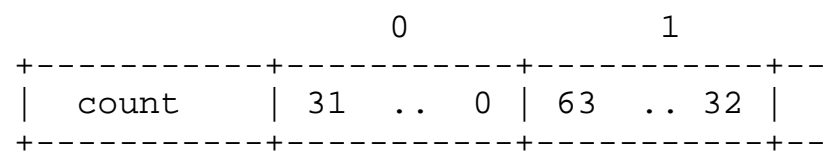

change_info4

struct change_info4
$\begin{aligned} & \text { bool } \\ & \text { changeid4 } \\ & \text { changeid4 }\end{aligned}$ $\begin{aligned} & \text { atomic; } \\ & \text { before; } \\ & \text { after; }\end{aligned}$

This structure is used with the CREATE, LINK, REMOVE, RENAME operations to let the client know the value of the change attribute for the directory in which the target filesystem object resides.

clientaddr 4

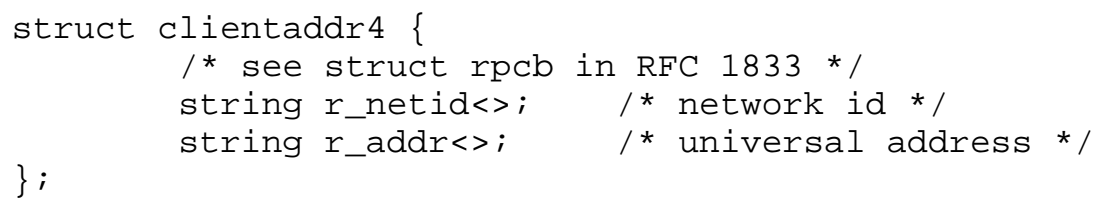

The clientaddr4 structure is used as part of the SETCLIENTID operation to either specify the address of the client that is using a clientid or as part of the callback registration. The 
r_netid and r_addr fields are specified in [RFC1833], but they are underspecified in [RFC1833] as far as what they should look like for specific protocols.

For TCP over IPV4 and for UDP over IPV4, the format of r_addr is the US-ASCII string:

$$
\text { h1.h2.h3.h4.p1.p2 }
$$

The prefix, "h1.h2.h3.h4", is the standard textual form for representing an IPv4 address, which is always four octets long. Assuming big-endian ordering, h1, h2, h3, and h4, are respectively, the first through fourth octets each converted to ASCII-decimal. Assuming big-endian ordering, p1 and p2 are, respectively, the first and second octets each converted to ASCII-decimal. For example, if a host, in big-endian order, has an address of 0x0A010307 and there is a service listening on, in big endian order, port 0x020F (decimal 527), then the complete universal address is "10.1.3.7.2.15".

For TCP over IPv4 the value of r_netid is the string "tcp". For UDP over IPv4 the value of r_netid is the string "udp".

For TCP over IPV6 and for UDP over IPV6, the format of r_addr is the US-ASCII string:

$$
\mathrm{x} 1: \mathrm{x} 2: \mathrm{x} 3: \mathrm{x} 4: \mathrm{x} 5: \mathrm{x} 6: \mathrm{x} 7: \mathrm{x} 8 \cdot \mathrm{p} 1 \cdot \mathrm{p} 2
$$

The suffix "p1.p2" is the service port, and is computed the same way as with universal addresses for TCP and UDP over IPv4. The prefix, "x1:x2:x3:x4:x5:x6:x7:x8", is the standard textual form for representing an IPv6 address as defined in section 2.2 of [RFC2373]. Additionally, the two alternative forms specified in section 2.2 of [RFC2373] are also acceptable.

For TCP over IPv6 the value of r_netid is the string "tcp6". For UDP over IPv6 the value of r_netid is the string "udp6".

cb_client 4

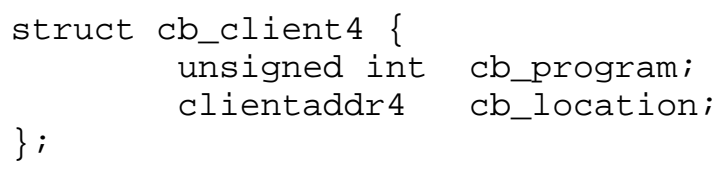

This structure is used by the client to inform the server of its call back address; includes the program number and client address. 
nfs_client_id4

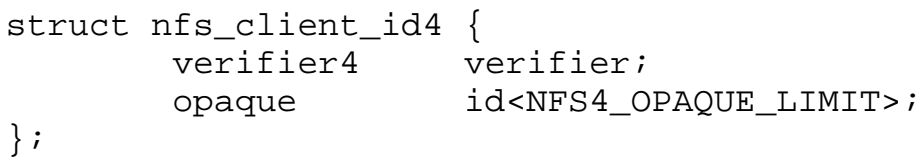

This structure is used for the first LOCK operation done for an open_owner4. It provides both the open_stateid and lock_owner such that the transition is made from a valid open_stateid sequence to that of the new lock_stateid sequence. Using this mechanism avoids the confirmation of the lock_owner/lock_seqid pair since it is tied to established state in the form of the open_stateid/open_seqid. 
stateid4

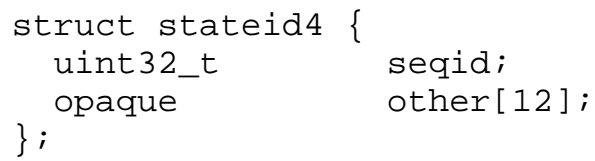

This structure is used for the various state sharing mechanisms between the client and server. For the client, this data structure is read-only. The starting value of the seqid field is undefined. The server is required to increment the seqid field monotonically at each transition of the stateid. This is important since the client will inspect the seqid in OPEN stateids to determine the order of OPEN processing done by the server.

3. RPC and Security Flavor

The NFS version 4 protocol is a Remote Procedure Call (RPC) application that uses RPC version 2 and the corresponding external Data Representation (XDR) as defined in [RFC1831] and [RFC1832]. The RPCSEC_GSS security flavor as defined in [RFC2203] MUST be used as the mechanism to deliver stronger security for the NFS version 4 protocol.

\subsection{Ports and Transports}

Historically, NFS version 2 and version 3 servers have resided on port 2049. The registered port 2049 [RFC3232] for the NFS protocol should be the default configuration. Using the registered port for NFS services means the NFS client will not need to use the RPC binding protocols as described in [RFC1833]; this will allow NFS to transit firewalls.

Where an NFS version 4 implementation supports operation over the IP network protocol, the supported transports between NFS and IP MUST be among the IETF-approved congestion control transport protocols, which include TCP and SCTP. To enhance the possibilities for interoperability, an NFS version 4 implementation MUST support operation over the TCP transport protocol, at least until such time as a standards track RFC revises this requirement to use a different IETF-approved congestion control transport protocol.

If TCP is used as the transport, the client and server SHOULD use persistent connections. This will prevent the weakening of $\mathrm{TCP}^{\prime} \mathrm{S}$ congestion control via short lived connections and will improve performance for the WAN environment by eliminating the need for SYN handshakes. 
As noted in the Security Considerations section, the authentication model for NFS version 4 has moved from machine-based to principalbased. However, this modification of the authentication model does not imply a technical requirement to move the TCP connection management model from whole machine-based to one based on a per user model. In particular, NFS over TCP client implementations have traditionally multiplexed traffic for multiple users over a common TCP connection between an NFS client and server. This has been true, regardless whether the NFS client is using AUTH_SYS, AUTH_DH, RPCSEC_GSS or any other flavor. Similarly, NFS over TCP server implementations have assumed such a model and thus scale the implementation of TCP connection management in proportion to the number of expected client machines. It is intended that NFS version 4 will not modify this connection management model. NFS version 4 clients that violate this assumption can expect scaling issues on the server and hence reduced service.

Note that for various timers, the client and server should avoid inadvertent synchronization of those timers. For further discussion of the general issue refer to [Floyd].

\subsubsection{Client Retransmission Behavior}

When processing a request received over a reliable transport such as TCP, the NFS version 4 server MUST NOT silently drop the request, except if the transport connection has been broken. Given such a contract between NFS version 4 clients and servers, clients MUST NOT retry a request unless one or both of the following are true:

- The transport connection has been broken

- The procedure being retried is the NULL procedure

Since reliable transports, such as TCP, do not always synchronously inform a peer when the other peer has broken the connection (for example, when an NFS server reboots), the NFS version 4 client may want to actively "probe" the connection to see if has been broken. Use of the NULL procedure is one recommended way to do so. So, when a client experiences a remote procedure call timeout (of some arbitrary implementation specific amount), rather than retrying the remote procedure call, it could instead issue a NULL procedure call to the server. If the server has died, the transport connection break will eventually be indicated to the NFS version 4 client. The client can then reconnect, and then retry the original request. If the NULL procedure call gets a response, the connection has not broken. The client can decide to wait longer for the original request's response, or it can break the transport connection and reconnect before re-sending the original request. 
For callbacks from the server to the client, the same rules apply, but the server doing the callback becomes the client, and the client receiving the callback becomes the server.

\subsection{Security Flavors}

Traditional RPC implementations have included AUTH_NONE, AUTH_SYS, AUTH_DH, and AUTH_KRB4 as security flavors. With [RFC2203] an additional security flavor of RPCSEC_GSS has been introduced which uses the functionality of GSS-API [RFC2743]. This allows for the use of various security mechanisms by the RPC layer without the additional implementation overhead of adding RPC security flavors. For NFS version 4, the RPCSEC_GSS security flavor MUST be used to enable the mandatory security mechanism. Other flavors, such as, AUTH_NONE, AUTH_SYS, and AUTH_DH MAY be implemented as well.

\subsubsection{Security mechanisms for NFS version 4}

The use of RPCSEC_GSS requires selection of: mechanism, quality of protection, and service (authentication, integrity, privacy). The remainder of this document will refer to these three parameters of the RPCSEC_GSS security as the security triple.

\subsubsection{Kerberos V5 as a security triple}

The Kerberos V5 GSS-API mechanism as described in [RFC1964] MUST be implemented and provide the following security triples.

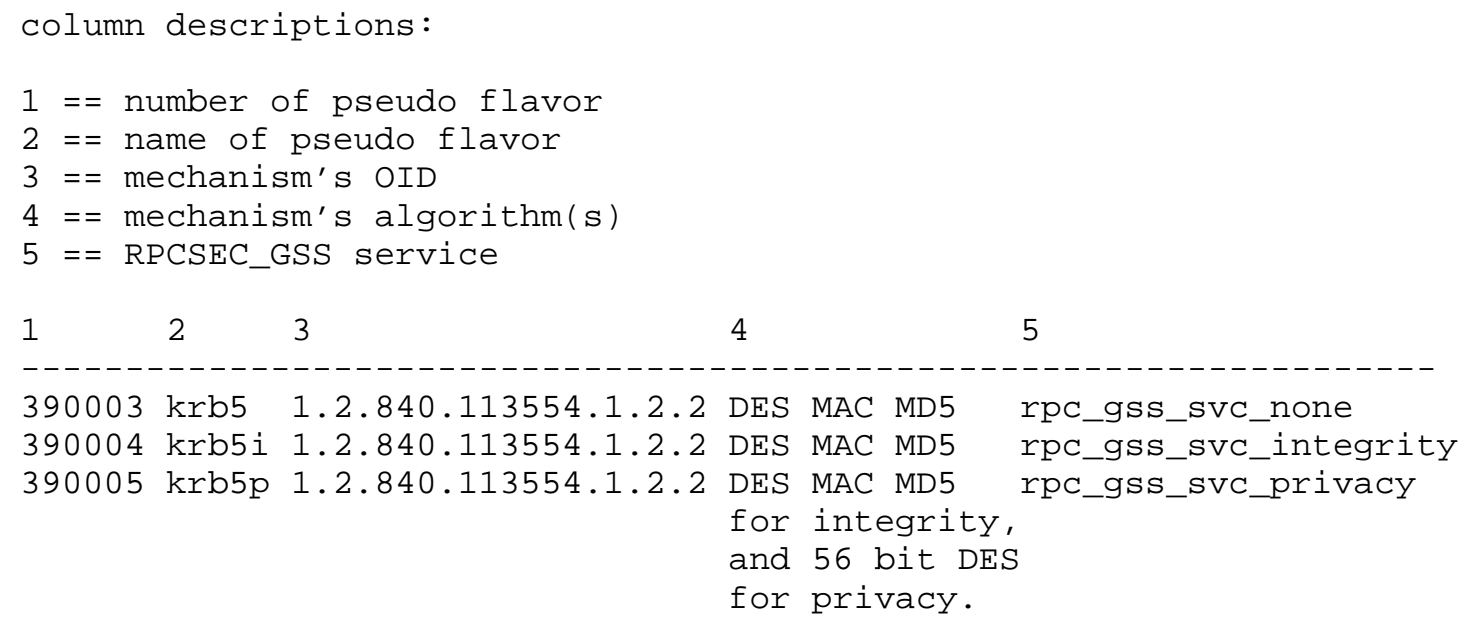

Note that the pseudo flavor is presented here as a mapping aid to the implementor. Because this NFS protocol includes a method to negotiate security and it understands the GSS-API mechanism, the 
pseudo flavor is not needed. The pseudo flavor is needed for NFS version 3 since the security negotiation is done via the MOUNT protocol.

For a discussion of NFS' use of RPCSEC_GSS and Kerberos V5, please see [RFC2623].

Users and implementors are warned that 56 bit DES is no longer considered state of the art in terms of resistance to brute force attacks. Once a revision to [RFC1964] is available that adds support for AES, implementors are urged to incorporate AES into their NFSV4 over Kerberos V5 protocol stacks, and users are similarly urged to migrate to the use of AES.

\subsubsection{LIPKEY as a security triple}

The LIPKEY GSS-API mechanism as described in [RFC2847] MUST be implemented and provide the following security triples. The definition of the columns matches the previous subsection "Kerberos v5 as security triple"

\begin{tabular}{|c|c|c|c|c|}
\hline 1 & 2 & 3 & 4 & 5 \\
\hline 390006 & lipkey & $1.3 \cdot 6 \cdot 1 \cdot 5 \cdot 5 \cdot 9$ & negotiated & rpc_gss_svc_n \\
\hline 390007 & lipkey-i & $1.3 \cdot 6 \cdot 1 \cdot 5 \cdot 5 \cdot 9$ & negotiated & rpc_gss_svc_integrity \\
\hline 390008 & lipkey-p & $1.3 \cdot 6 \cdot 1 \cdot 5 \cdot 5 \cdot 9$ & negotiated & rpc_gss_svc_privacy \\
\hline
\end{tabular}

The mechanism algorithm is listed as "negotiated". This is because LIPKEY is layered on SPKM-3 and in SPKM-3 [RFC2847] the confidentiality and integrity algorithms are negotiated. Since SPKM-3 specifies HMAC-MD5 for integrity as MANDATORY, 128 bit cast5CBC for confidentiality for privacy as MANDATORY, and further specifies that HMAC-MD5 and cast5CBC MUST be listed first before weaker algorithms, specifying "negotiated" in column 4 does not impair interoperability. In the event an SPKM-3 peer does not support the mandatory algorithms, the other peer is free to accept or reject the GSS-API context creation.

Because SPKM-3 negotiates the algorithms, subsequent calls to LIPKEY's GSS_Wrap() and GSS_GetMIC() by RPCSEC_GSS will use a quality of protection value of 0 (zero). See section 5.2 of [RFC2025] for an explanation.

LIPKEY uses SPKM-3 to create a secure channel in which to pass a user name and password from the client to the server. Once the user name and password have been accepted by the server, calls to the LIPKEY context are redirected to the SPKM-3 context. See [RFC2847] for more details. 


\subsubsection{SPKM-3 as a security triple}

The SPKM-3 GSS-API mechanism as described in [RFC2847] MUST be implemented and provide the following security triples. The definition of the columns matches the previous subsection "Kerberos v5 as security triple".

\begin{tabular}{|c|c|c|c|c|}
\hline 1 & 2 & 3 & 4 & 5 \\
\hline 390009 & spkm3 & 1.3 .6 .1 .5 .5 .1 .3 & negotiated & rpc_gss_svc_none \\
\hline 390010 & spkm3i & $1.3 .6 .1 .5 \cdot 5 \cdot 1.3$ & negotiated & rpc_gss_svc_integrity \\
\hline 390011 & spkm3p & 1.3 .6 .1 .5 .5 .1 .3 & negotiated & rpc_gss_svc_privacy \\
\hline
\end{tabular}

For a discussion as to why the mechanism algorithm is listed as "negotiated", see the previous section "LIPKEY as a security triple."

Because SPKM-3 negotiates the algorithms, subsequent calls to SPKM3's GSS_Wrap() and GSS_GetMIC() by RPCSEC_GSS will use a quality of protection value of 0 (zero). See section 5.2 of [RFC2025] for an explanation.

Even though LIPKEY is layered over SPKM-3, SPKM-3 is specified as a mandatory set of triples to handle the situations where the initiator (the client) is anonymous or where the initiator has its own certificate. If the initiator is anonymous, there will not be a user name and password to send to the target (the server). If the initiator has its own certificate, then using passwords is superfluous.

\subsection{Security Negotiation}

With the NFS version 4 server potentially offering multiple security mechanisms, the client needs a method to determine or negotiate which mechanism is to be used for its communication with the server. The NFS server may have multiple points within its filesystem name space that are available for use by NFS clients. In turn the NFS server may be configured such that each of these entry points may have different or multiple security mechanisms in use.

The security negotiation between client and server must be done with a secure channel to eliminate the possibility of a third party intercepting the negotiation sequence and forcing the client and server to choose a lower level of security than required or desired. See the section "Security Considerations" for further discussion. 


\subsubsection{SECINFO}

The new SECINFO operation will allow the client to determine, on a per filehandle basis, what security triple is to be used for server access. In general, the client will not have to use the SECINFO operation except during initial communication with the server or when the client crosses policy boundaries at the server. It is possible that the server's policies change during the client's interaction therefore forcing the client to negotiate a new security triple.

\subsubsection{Security Error}

Based on the assumption that each NFS version 4 client and server must support a minimum set of security (i.e., LIPKEY, SPKM-3, and Kerberos-V5 all under RPCSEC_GSS), the NFS client will start its communication with the server with one of the minimal security triples. During communication with the server, the client may receive an NFS error of NFS4ERR_WRONGSEC. This error allows the server to notify the client that the security triple currently being used is not appropriate for access to the server's filesystem resources. The client is then responsible for determining what security triples are available at the server and choose one which is appropriate for the client. See the section for the "SECINFO" operation for further discussion of how the client will respond to the NFS4ERR_WRONGSEC error and use SECINFO.

\subsection{Callback RPC Authentication}

Except as noted elsewhere in this section, the callback RPC (described later) MUST mutually authenticate the NFS server to the principal that acquired the clientid (also described later), using the security flavor the original SETCLIENTID operation used.

For AUTH_NONE, there are no principals, so this is a non-issue.

AUTH_SYS has no notions of mutual authentication or a server principal, so the callback from the server simply uses the AUTH_SYS credential that the user used when he set up the delegation.

For AUTH_DH, one commonly used convention is that the server uses the credential corresponding to this AUTH_DH principal:

$$
\text { unix.host@domain }
$$

where host and domain are variables corresponding to the name of server host and directory services domain in which it lives such as a Network Information system domain or a DNS domain. 
Because LIPKEY is layered over SPKM-3, it is permissible for the server to use SPKM-3 and not LIPKEY for the callback even if the client used LIPKEY for SETCLIENTID.

Regardless of what security mechanism under RPCSEC_GSS is being used, the NFS server, MUST identify itself in GSS-API via a GSS_C_NT_HOSTBASED_SERVICE name type. GSS_C_NT_HOSTBASED_SERVICE names are of the form:

service@hostname

For NFS, the "service" element is

$n f s$

Implementations of security mechanisms will convert nfs@hostname to various different forms. For Kerberos V5 and LIPKEY, the following form is RECOMMENDED:

\section{nfs/hostname}

For Kerberos V5, nfs/hostname would be a server principal in the Kerberos Key Distribution Center database. This is the same principal the client acquired a GSS-API context for when it issued the SETCLIENTID operation, therefore, the realm name for the server principal must be the same for the callback as it was for the SETCLIENTID.

For LIPKEY, this would be the username passed to the target (the NFS version 4 client that receives the callback).

It should be noted that LIPKEY may not work for callbacks, since the LIPKEY client uses a user id/password. If the NFS client receiving the callback can authenticate the NFS server's user name/password pair, and if the user that the NFS server is authenticating to has a public key certificate, then it works.

In situations where the NFS client uses LIPKEY and uses a per-host principal for the SETCLIENTID operation, instead of using LIPKEY for SETCLIENTID, it is RECOMMENDED that SPKM-3 with mutual authentication be used. This effectively means that the client will use a certificate to authenticate and identify the initiator to the target on the NFS server. Using SPKM-3 and not LIPKEY has the following advantages:

- When the server does a callback, it must authenticate to the principal used in the SETCLIENTID. Even if LIPKEY is used, because LIPKEY is layered over SPKM-3, the NFS client will need to 
have a certificate that corresponds to the principal used in the SETCLIENTID operation. From an administrative perspective, having a user name, password, and certificate for both the client and server is redundant.

- LIPKEY was intended to minimize additional infrastructure requirements beyond a certificate for the target, and the expectation is that existing password infrastructure can be leveraged for the initiator. In some environments, a per-host password does not exist yet. If certificates are used for any per-host principals, then additional password infrastructure is not needed.

- In cases when a host is both an NFS client and server, it can share the same per-host certificate.

4. Filehandles

The filehandle in the NFS protocol is a per server unique identifier for a filesystem object. The contents of the filehandle are opaque to the client. Therefore, the server is responsible for translating the filehandle to an internal representation of the filesystem object.

\subsection{Obtaining the First Filehandle}

The operations of the NFS protocol are defined in terms of one or more filehandles. Therefore, the client needs a filehandle to initiate communication with the server. With the NFS version 2 protocol [RFC1094] and the NFS version 3 protocol [RFC1813], there exists an ancillary protocol to obtain this first filehandle. The MOUNT protocol, RPC program number 100005, provides the mechanism of translating a string based filesystem path name to a filehandle which can then be used by the NFS protocols.

The MOUNT protocol has deficiencies in the area of security and use via firewalls. This is one reason that the use of the public filehandle was introduced in [RFC2054] and [RFC2055]. With the use of the public filehandle in combination with the LOOKUP operation in the NFS version 2 and 3 protocols, it has been demonstrated that the MOUNT protocol is unnecessary for viable interaction between NFS client and server.

Therefore, the NFS version 4 protocol will not use an ancillary protocol for translation from string based path names to a filehandle. Two special filehandles will be used as starting points for the NFS client. 


\subsubsection{Root Filehandle}

The first of the special filehandles is the ROOT filehandle. The ROOT filehandle is the "conceptual" root of the filesystem name space at the NFS server. The client uses or starts with the ROOT filehandle by employing the PUTROOTFH operation. The PUTROOTFH operation instructs the server to set the "current" filehandle to the ROOT of the server's file tree. Once this PUTROOTFH operation is used, the client can then traverse the entirety of the server's file tree with the LoOKUP operation. A complete discussion of the server name space is in the section "NFS Server Name space".

\subsubsection{Public Filehandle}

The second special filehandle is the PUBLIC filehandle. Unlike the ROOT filehandle, the PUBLIC filehandle may be bound or represent an arbitrary filesystem object at the server. The server is responsible for this binding. It may be that the PUBLIC filehandle and the ROOT filehandle refer to the same filesystem object. However, it is up to the administrative software at the server and the policies of the server administrator to define the binding of the PUBLIC filehandle and server filesystem object. The client may not make any assumptions about this binding. The client uses the PUBLIC filehandle via the PUTPUBFH operation.

\subsection{Filehandle Types}

In the NFS version 2 and 3 protocols, there was one type of filehandle with a single set of semantics. This type of filehandle is termed "persistent" in NFS Version 4. The semantics of a persistent filehandle remain the same as before. A new type of filehandle introduced in NFS Version 4 is the "volatile" filehandle, which attempts to accommodate certain server environments.

The volatile filehandle type was introduced to address server functionality or implementation issues which make correct implementation of a persistent filehandle infeasible. Some server environments do not provide a filesystem level invariant that can be used to construct a persistent filehandle. The underlying server filesystem may not provide the invariant or the server's filesystem programming interfaces may not provide access to the needed invariant. Volatile filehandles may ease the implementation of server functionality such as hierarchical storage management or filesystem reorganization or migration. However, the volatile filehandle increases the implementation burden for the client. 
Since the client will need to handle persistent and volatile filehandles differently, a file attribute is defined which may be used by the client to determine the filehandle types being returned by the server.

\subsubsection{General Properties of a Filehandle}

The filehandle contains all the information the server needs to distinguish an individual file. To the client, the filehandle is opaque. The client stores filehandles for use in a later request and can compare two filehandles from the same server for equality by doing a byte-by-byte comparison. However, the client MUST NOT otherwise interpret the contents of filehandles. If two filehandles from the same server are equal, they MUST refer to the same file. Servers SHOULD try to maintain a one-to-one correspondence between filehandles and files but this is not required. Clients MUST use filehandle comparisons only to improve performance, not for correct behavior. All clients need to be prepared for situations in which it cannot be determined whether two filehandles denote the same object and in such cases, avoid making invalid assumptions which might cause incorrect behavior. Further discussion of filehandle and attribute comparison in the context of data caching is presented in the section "Data Caching and File Identity".

As an example, in the case that two different path names when traversed at the server terminate at the same filesystem object, the server SHOULD return the same filehandle for each path. This can occur if a hard link is used to create two file names which refer to the same underlying file object and associated data. For example, if paths /a/b/c and /a/d/c refer to the same file, the server SHOULD return the same filehandle for both path names traversals.

\subsubsection{Persistent Filehandle}

A persistent filehandle is defined as having a fixed value for the lifetime of the filesystem object to which it refers. Once the server creates the filehandle for a filesystem object, the server MUST accept the same filehandle for the object for the lifetime of the object. If the server restarts or reboots the NFS server must honor the same filehandle value as it did in the server's previous instantiation. Similarly, if the filesystem is migrated, the new NFS server must honor the same filehandle as the old NFS server.

The persistent filehandle will be become stale or invalid when the filesystem object is removed. When the server is presented with a persistent filehandle that refers to a deleted object, it MUST return an error of NFS4ERR_STALE. A filehandle may become stale when the filesystem containing the object is no longer available. The file 
system may become unavailable if it exists on removable media and the media is no longer available at the server or the filesystem in whole has been destroyed or the filesystem has simply been removed from the server's name space (i.e., unmounted in a UNIX environment).

\subsubsection{Volatile Filehandle}

A volatile filehandle does not share the same longevity characteristics of a persistent filehandle. The server may determine that a volatile filehandle is no longer valid at many different points in time. If the server can definitively determine that a volatile filehandle refers to an object that has been removed, the server should return NFS4ERR_STALE to the client (as is the case for persistent filehandles). In all other cases where the server determines that a volatile filehandle can no longer be used, it should return an error of NFS4ERR_FHEXPIRED.

The mandatory attribute "fh_expire_type" is used by the client to determine what type of filehandle the server is providing for a particular filesystem. This attribute is a bitmask with the following values:

FH4_PERSISTENT

The value of FH4_PERSISTENT is used to indicate a persistent filehandle, which is valid until the object is removed from the filesystem. The server will not return NFS4ERR_FHEXPIRED for this filehandle. FH4_PERSISTENT is defined as a value in which none of the bits specified below are set.

FH4_VOLATILE_ANY

The filehandle may expire at any time, except as specifically excluded (i.e., FH4_NO_EXPIRE_WITH_OPEN) .

FH4_NOEXPIRE_WITH_OPEN

May only be set when FH4_VOLATILE_ANY is set. If this bit is set, then the meaning of FH4_VOLATILE_ANY is qualified to exclude any expiration of the filehandle when it is open.

FH4_VOL_MIGRATION

The filehandle will expire as a result of migration. If FH4_VOL_ANY is set, FH4_VOL_MIGRATION is redundant. 


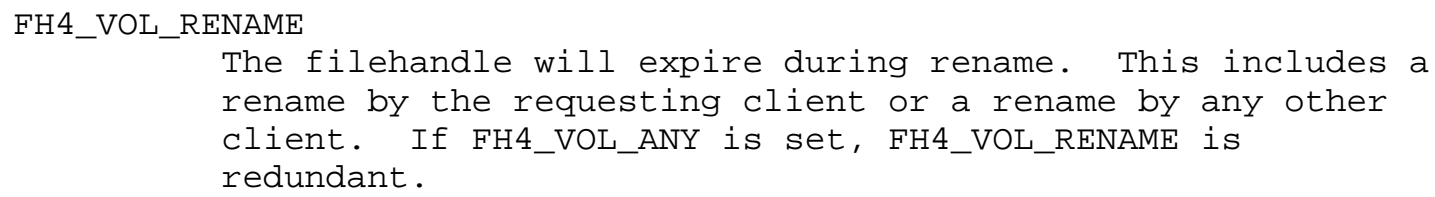

Servers which provide volatile filehandles that may expire while open (i.e., if FH4_VOL_MIGRATION or FH4_VOL_RENAME is set or if

FH4_VOLATILE_ANY is set and FH4_NOEXPIRE_WITH_OPEN not set), should deny a RENAME or REMOVE that would affect an OPEN file of any of the components leading to the OPEN file. In addition, the server should deny all RENAME or REMOVE requests during the grace period upon server restart.

Note that the bits FH4_VOL_MIGRATION and FH4_VOL_RENAME allow the client to determine that expiration has occurred whenever a specific event occurs, without an explicit filehandle expiration error from the server. FH4_VOL_ANY does not provide this form of information. In situations where the server will expire many, but not all filehandles upon migration (e.g., all but those that are open), FH4_VOLATILE_ANY (in this case with FH4_NOEXPIRE_WITH_OPEN) is a better choice since the client may not assume that all filehandles will expire when migration occurs, and it is likely that additional expirations will occur (as a result of file CLOSE) that are separated in time from the migration event itself.

4.2.4. One Method of Constructing a Volatile Filehandle

A volatile filehandle, while opaque to the client could contain:

[volatile bit $=1 \mid$ server boot time $\mid$ slot $\mid$ generation number]

o slot is an index in the server volatile filehandle table

o generation number is the generation number for the table entry/slot

When the client presents a volatile filehandle, the server makes the following checks, which assume that the check for the volatile bit has passed. If the server boot time is less than the current server boot time, return NFS4ERR_FHEXPIRED. If slot is out of range, return NFS4ERR_BADHANDLE. If the generation number does not match, return NFS 4ERR_FHEXPIRED.

When the server reboots, the table is gone (it is volatile).

If volatile bit is 0 , then it is a persistent filehandle with a different structure following it. 


\subsection{Client Recovery from Filehandle Expiration}

If possible, the client SHOULD recover from the receipt of an NFS4ERR_FHEXPIRED error. The client must take on additional responsibility so that it may prepare itself to recover from the expiration of a volatile filehandle. If the server returns persistent filehandles, the client does not need these additional steps.

For volatile filehandles, most commonly the client will need to store the component names leading up to and including the filesystem object in question. With these names, the client should be able to recover by finding a filehandle in the name space that is still available or by starting at the root of the server's filesystem name space.

If the expired filehandle refers to an object that has been removed from the filesystem, obviously the client will not be able to recover from the expired filehandle.

It is also possible that the expired filehandle refers to a file that has been renamed. If the file was renamed by another client, again it is possible that the original client will not be able to recover. However, in the case that the client itself is renaming the file and the file is open, it is possible that the client may be able to recover. The client can determine the new path name based on the processing of the rename request. The client can then regenerate the new filehandle based on the new path name. The client could also use the compound operation mechanism to construct a set of operations like:

RENAME A B

LOOKUP B

GETFH

Note that the COMPOUND procedure does not provide atomicity. This example only reduces the overhead of recovering from an expired filehandle.

\section{File Attributes}

To meet the requirements of extensibility and increased interoperability with non-UNIX platforms, attributes must be handled in a flexible manner. The NFS version 3 fattr3 structure contains a fixed list of attributes that not all clients and servers are able to support or care about. The fattr3 structure can not be extended as new needs arise and it provides no way to indicate non-support. With the NFS version 4 protocol, the client is able query what attributes the server supports and construct requests with only those supported attributes (or a subset thereof). 
To this end, attributes are divided into three groups: mandatory, recommended, and named. Both mandatory and recommended attributes are supported in the NFS version 4 protocol by a specific and welldefined encoding and are identified by number. They are requested by setting a bit in the bit vector sent in the GETATTR request; the server response includes a bit vector to list what attributes were returned in the response. New mandatory or recommended attributes may be added to the NFS protocol between major revisions by publishing a standards-track RFC which allocates a new attribute number value and defines the encoding for the attribute. See the section "Minor Versioning" for further discussion.

Named attributes are accessed by the new OPENATTR operation, which accesses a hidden directory of attributes associated with a file system object. OPENATTR takes a filehandle for the object and returns the filehandle for the attribute hierarchy. The filehandle for the named attributes is a directory object accessible by LOOKUP or READDIR and contains files whose names represent the named attributes and whose data bytes are the value of the attribute. For example:

$\begin{array}{lll}\text { LOOKUP } & \text { "foo" } & ; \text { look up file } \\ \text { GETATTR } & \text { attrbits } & \\ \text { OPENATTR } & & \text {; access foo's named attributes } \\ \text { LOOKUP } & \text { "x11icon" } & \text {; look up specific attribute } \\ \text { READ } & 0,4096 & ; \text { read stream of bytes }\end{array}$

Named attributes are intended for data needed by applications rather than by an NFS client implementation. NFS implementors are strongly encouraged to define their new attributes as recommended attributes by bringing them to the IETF standards-track process.

The set of attributes which are classified as mandatory is deliberately small since servers must do whatever it takes to support them. A server should support as many of the recommended attributes as possible but by their definition, the server is not required to support all of them. Attributes are deemed mandatory if the data is both needed by a large number of clients and is not otherwise reasonably computable by the client when support is not provided on the server.

Note that the hidden directory returned by OPENATTR is a convenience for protocol processing. The client should not make any assumptions about the server's implementation of named attributes and whether the underlying filesystem at the server has a named attribute directory or not. Therefore, operations such as SETATTR and GETATTR on the named attribute directory are undefined. 


\subsection{Mandatory Attributes}

These MUST be supported by every NFS version 4 client and server in order to ensure a minimum level of interoperability. The server must store and return these attributes and the client must be able to function with an attribute set limited to these attributes. With just the mandatory attributes some client functionality may be impaired or limited in some ways. A client may ask for any of these attributes to be returned by setting a bit in the GETATTR request and the server must return their value.

\subsection{Recommended Attributes}

These attributes are understood well enough to warrant support in the NFS version 4 protocol. However, they may not be supported on all clients and servers. A client may ask for any of these attributes to be returned by setting a bit in the GETATTR request but must handle the case where the server does not return them. A client may ask for the set of attributes the server supports and should not request attributes the server does not support. A server should be tolerant of requests for unsupported attributes and simply not return them rather than considering the request an error. It is expected that servers will support all attributes they comfortably can and only fail to support attributes which are difficult to support in their operating environments. A server should provide attributes whenever they don't have to "tell lies" to the client. For example, a file modification time should be either an accurate time or should not be supported by the server. This will not always be comfortable to clients but the client is better positioned decide whether and how to fabricate or construct an attribute or whether to do without the attribute.

\subsection{Named Attributes}

These attributes are not supported by direct encoding in the NFS Version 4 protocol but are accessed by string names rather than numbers and correspond to an uninterpreted stream of bytes which are stored with the filesystem object. The name space for these attributes may be accessed by using the OPENATTR operation. The OPENATTR operation returns a filehandle for a virtual "attribute directory" and further perusal of the name space may be done using READDIR and LOOKUP operations on this filehandle. Named attributes may then be examined or changed by normal READ and WRITE and CREATE operations on the filehandles returned from READDIR and LOOKUP. Named attributes may have attributes. 
It is recommended that servers support arbitrary named attributes. A client should not depend on the ability to store any named attributes in the server's filesystem. If a server does support named attributes, a client which is also able to handle them should be able to copy a file's data and meta-data with complete transparency from one location to another; this would imply that names allowed for regular directory entries are valid for named attribute names as well.

Names of attributes will not be controlled by this document or other IETF standards track documents. See the section "IANA Considerations" for further discussion.

\subsection{Classification of Attributes}

Each of the Mandatory and Recommended attributes can be classified in one of three categories: per server, per filesystem, or per filesystem object. Note that it is possible that some per filesystem attributes may vary within the filesystem. See the "homogeneous" attribute for its definition. Note that the attributes time_access_set and time_modify_set are not listed in this section because they are write-only attributes corresponding to time_access and time_modify, and are used in a special instance of SETATTR.

- The per server attribute is:

$$
\text { lease_time }
$$

o The per filesystem attributes are:

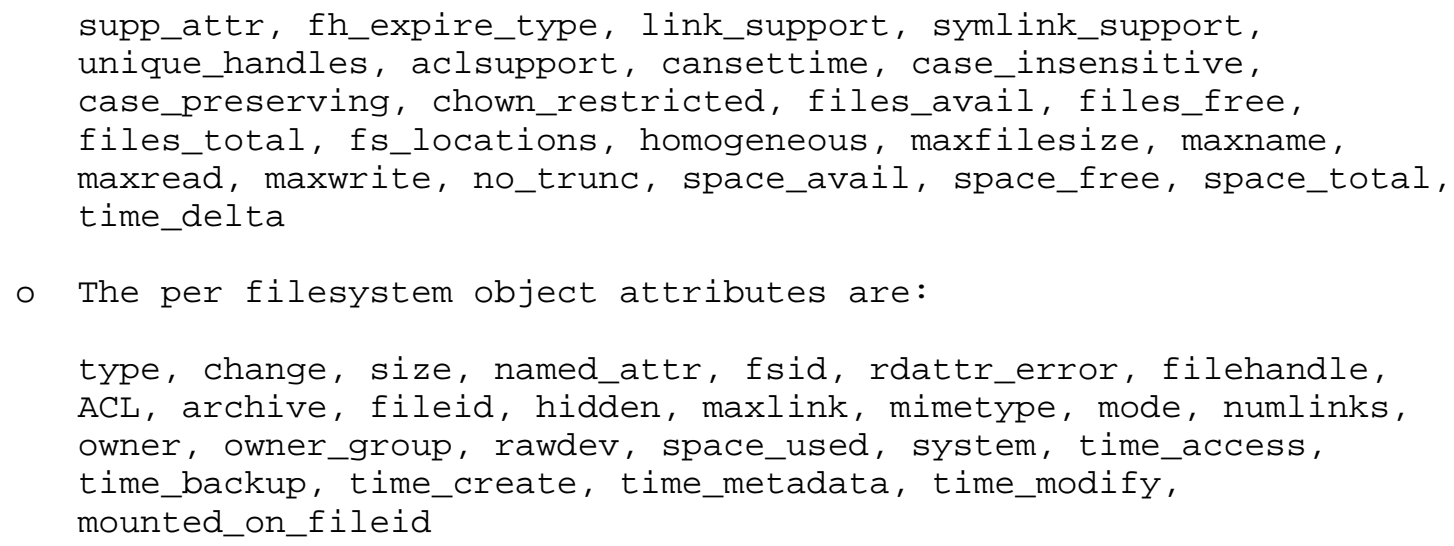

For quota_avail_hard, quota_avail_soft, and quota_used see their definitions below for the appropriate classification. 
5.5. Mandatory Attributes - Definitions

\begin{tabular}{|c|c|c|c|c|}
\hline Name & $\#$ & DataType & Access & Description \\
\hline supp_attr & 0 & bitmap & READ & $\begin{array}{l}\text { The bit vector which } \\
\text { would retrieve all } \\
\text { mandatory and } \\
\text { recommended attributes } \\
\text { that are supported for } \\
\text { this object. The } \\
\text { scope of this } \\
\text { attribute applies to } \\
\text { all objects with a } \\
\text { matching fsid. }\end{array}$ \\
\hline type & 1 & nfs4_ftype & READ & $\begin{array}{l}\text { The type of the object } \\
\text { (file, directory, } \\
\text { symlink, etc.) }\end{array}$ \\
\hline fh_expire_type & 2 & uint 32 & READ & $\begin{array}{l}\text { Server uses this to } \\
\text { specify filehandle } \\
\text { expiration behavior to } \\
\text { the client. See the } \\
\text { section "Filehandles" } \\
\text { for additional } \\
\text { description. }\end{array}$ \\
\hline change & 3 & uint 64 & READ & $\begin{array}{l}\text { A value created by the } \\
\text { server that the client } \\
\text { can use to determine } \\
\text { if file data, } \\
\text { directory contents or } \\
\text { attributes of the } \\
\text { object have been } \\
\text { modified. The server } \\
\text { may return the } \\
\text { object's time_metadata } \\
\text { attribute for this } \\
\text { attribute's value but } \\
\text { only if the filesystem } \\
\text { object can not be } \\
\text { updated more } \\
\text { frequently than the } \\
\text { resolution of } \\
\text { time_metadata. }\end{array}$ \\
\hline size & 4 & uint 64 & $\mathrm{R} / \mathrm{W}$ & $\begin{array}{l}\text { The size of the object } \\
\text { in bytes. }\end{array}$ \\
\hline
\end{tabular}




\begin{tabular}{|c|c|c|c|c|}
\hline link_support & 5 & bool & READ & $\begin{array}{l}\text { True, if the object's } \\
\text { filesystem supports } \\
\text { hard links. }\end{array}$ \\
\hline symlink_support & 6 & bool & READ & $\begin{array}{l}\text { True, if the object's } \\
\text { filesystem supports } \\
\text { symbolic links. }\end{array}$ \\
\hline named_attr & 7 & bool & READ & $\begin{array}{l}\text { True, if this object } \\
\text { has named attributes. } \\
\text { In other words, object } \\
\text { has a non-empty named } \\
\text { attribute directory. }\end{array}$ \\
\hline fsid & 8 & fsid4 & READ & $\begin{array}{l}\text { Unique filesystem } \\
\text { identifier for the } \\
\text { filesystem holding } \\
\text { this object. fsid } \\
\text { contains major and } \\
\text { minor components each } \\
\text { of which are uint } 64 .\end{array}$ \\
\hline unique_handles & 9 & bool & READ & $\begin{array}{l}\text { True, if two distinct } \\
\text { filehandles guaranteed } \\
\text { to refer to two } \\
\text { different filesystem } \\
\text { objects. }\end{array}$ \\
\hline lease_time & 10 & nfs_lease 4 & READ & $\begin{array}{l}\text { Duration of leases at } \\
\text { server in seconds. }\end{array}$ \\
\hline rdattr_error & 11 & enum & READ & $\begin{array}{l}\text { Error returned from } \\
\text { getattr during } \\
\text { readdir. }\end{array}$ \\
\hline filehandle & 19 & nfs_fh 4 & READ & $\begin{array}{l}\text { The filehandle of this } \\
\text { object (primarily for } \\
\text { readdir requests). }\end{array}$ \\
\hline
\end{tabular}


5.6. Recommended Attributes - Definitions

\begin{tabular}{|c|c|c|c|c|}
\hline Name & $\#$ & Data Type & Access & Description \\
\hline ACL & 12 & nfsace $4<>$ & $\mathrm{R} / \mathrm{W}$ & $\begin{array}{l}\text { The access control } \\
\text { list for the object. }\end{array}$ \\
\hline aclsupport & 13 & uint 32 & READ & $\begin{array}{l}\text { Indicates what types } \\
\text { of ACLs are } \\
\text { supported on the } \\
\text { current filesystem. }\end{array}$ \\
\hline archive & 14 & bool & $\mathrm{R} / \mathrm{W}$ & $\begin{array}{l}\text { True, if this file } \\
\text { has been archived } \\
\text { since the time of } \\
\text { last modification } \\
\text { (deprecated in favor } \\
\text { of time_backup). }\end{array}$ \\
\hline cansettime & 15 & $\mathrm{bool}$ & READ & $\begin{array}{l}\text { True, if the server } \\
\text { is able to change } \\
\text { the times for a } \\
\text { filesystem object as } \\
\text { specified in a } \\
\text { SETATTR operation. }\end{array}$ \\
\hline case_insensitive & 16 & bool & READ & $\begin{array}{l}\text { True, if filename } \\
\text { comparisons on this } \\
\text { filesystem are case } \\
\text { insensitive. }\end{array}$ \\
\hline case_preserving & 17 & bool & READ & $\begin{array}{l}\text { True, if filename } \\
\text { case on this } \\
\text { filesystem are } \\
\text { preserved. }\end{array}$ \\
\hline chown_restricted & 18 & bool & READ & $\begin{array}{l}\text { If TRUE, the server } \\
\text { will reject any } \\
\text { request to change } \\
\text { either the owner or } \\
\text { the group associated } \\
\text { with a file if the } \\
\text { caller is not a } \\
\text { privileged user (for } \\
\text { example, "root" in } \\
\text { UNIX operating } \\
\text { environments or in } \\
\text { Windows } 2000 \text { the }\end{array}$ \\
\hline
\end{tabular}




\begin{tabular}{|c|c|c|c|c|}
\hline & & & & $\begin{array}{l}\text { "Take Ownership" } \\
\text { privilege). }\end{array}$ \\
\hline fileid & 20 & uint 64 & $\mathrm{READ}$ & $\begin{array}{l}\text { A number uniquely } \\
\text { identifying the file } \\
\text { within the } \\
\text { filesystem. }\end{array}$ \\
\hline files_avail & 21 & uint 64 & $\mathrm{READ}$ & $\begin{array}{l}\text { File slots available } \\
\text { to this user on the } \\
\text { filesystem } \\
\text { containing this } \\
\text { object - this should } \\
\text { be the smallest } \\
\text { relevant limit. }\end{array}$ \\
\hline files_free & 22 & uint 64 & READ & $\begin{array}{l}\text { Free file slots on } \\
\text { the filesystem } \\
\text { containing this } \\
\text { object - this should } \\
\text { be the smallest } \\
\text { relevant limit. }\end{array}$ \\
\hline files_total & 23 & uint 64 & READ & $\begin{array}{l}\text { Total file slots on } \\
\text { the filesystem } \\
\text { containing this } \\
\text { object. }\end{array}$ \\
\hline fs_locations & 24 & fs_locations & READ & $\begin{array}{l}\text { Locations where this } \\
\text { filesystem may be } \\
\text { found. If the } \\
\text { server returns } \\
\text { NFS4ERR_MOVED } \\
\text { as an error, this } \\
\text { attribute MUST be } \\
\text { supported. }\end{array}$ \\
\hline hidden & 25 & bool & $\mathrm{R} / \mathrm{W}$ & $\begin{array}{l}\text { True, if the file is } \\
\text { considered hidden } \\
\text { with respect to the } \\
\text { Windows API. }\end{array}$ \\
\hline homogeneous & 26 & bool & READ & $\begin{array}{l}\text { True, if this } \\
\text { object's filesystem } \\
\text { is homogeneous, } \\
\text { i.e., are per } \\
\text { filesystem } \\
\text { attributes the same }\end{array}$ \\
\hline
\end{tabular}




\begin{tabular}{|c|c|c|c|c|}
\hline & & & & $\begin{array}{l}\text { for all filesystem's } \\
\text { objects? }\end{array}$ \\
\hline maxfilesize & 27 & uint 64 & READ & $\begin{array}{l}\text { Maximum supported } \\
\text { file size for the } \\
\text { filesystem of this } \\
\text { object. }\end{array}$ \\
\hline $\operatorname{maxlink}$ & 28 & uint 32 & READ & $\begin{array}{l}\text { Maximum number of } \\
\text { links for this } \\
\text { object. }\end{array}$ \\
\hline maxname & 29 & uint 32 & $\mathrm{READ}$ & $\begin{array}{l}\text { Maximum filename } \\
\text { size supported for } \\
\text { this object. }\end{array}$ \\
\hline maxread & 30 & uint 64 & $\mathrm{READ}$ & $\begin{array}{l}\text { Maximum read size } \\
\text { supported for this } \\
\text { object. }\end{array}$ \\
\hline maxwrite & 31 & uint 64 & READ & $\begin{array}{l}\text { Maximum write size } \\
\text { supported for this } \\
\text { object. This } \\
\text { attribute SHould be } \\
\text { supported if the } \\
\text { file is writable. } \\
\text { Lack of this } \\
\text { attribute can } \\
\text { lead to the client } \\
\text { either wasting } \\
\text { bandwidth or not } \\
\text { receiving the best } \\
\text { performance. }\end{array}$ \\
\hline mimetype & 32 & utf $8<>$ & $\mathrm{R} / \mathrm{W}$ & $\begin{array}{l}\text { MIME body } \\
\text { type/subtype of this } \\
\text { object. }\end{array}$ \\
\hline mode & 33 & mode 4 & $\mathrm{R} / \mathrm{W}$ & $\begin{array}{l}\text { UNIX-style mode and } \\
\text { permission bits for } \\
\text { this object. }\end{array}$ \\
\hline no_trunc & 34 & bool & READ & $\begin{array}{l}\text { True, if a name } \\
\text { longer than name_max } \\
\text { is used, an error be } \\
\text { returned and name is } \\
\text { not truncated. }\end{array}$ \\
\hline
\end{tabular}




\begin{tabular}{|c|c|c|c|c|}
\hline numlinks & 35 & uint 32 & READ & $\begin{array}{l}\text { Number of hard links } \\
\text { to this object. }\end{array}$ \\
\hline owner & 36 & utf $8<>$ & $\mathrm{R} / \mathrm{W}$ & $\begin{array}{l}\text { The string name of } \\
\text { the owner of this } \\
\text { object. }\end{array}$ \\
\hline owner_group & 37 & ut $f 8<>$ & $\mathrm{R} / \mathrm{W}$ & $\begin{array}{l}\text { The string name of } \\
\text { the group ownership } \\
\text { of this object. }\end{array}$ \\
\hline quota_avail_hard & 38 & uint 64 & READ & $\begin{array}{l}\text { For definition see } \\
\text { "Quota Attributes" } \\
\text { section below. }\end{array}$ \\
\hline quota_avail_soft & 39 & uint 64 & READ & $\begin{array}{l}\text { For definition see } \\
\text { "Quota Attributes" } \\
\text { section below. }\end{array}$ \\
\hline quota_used & 40 & uint 64 & READ & $\begin{array}{l}\text { For definition see } \\
\text { "Quota Attributes" } \\
\text { section below. }\end{array}$ \\
\hline rawdev & 41 & specdata 4 & $\mathrm{READ}$ & $\begin{array}{l}\text { Raw device } \\
\text { identifier. UNIX } \\
\text { device major/minor } \\
\text { node information. } \\
\text { If the value of } \\
\text { type is not } \\
\text { NF4BLK or NF4CHR, } \\
\text { the value return } \\
\text { SHOULD NOT be } \\
\text { considered useful. }\end{array}$ \\
\hline space_avail & 42 & uint 64 & READ & $\begin{array}{l}\text { Disk space in bytes } \\
\text { available to this } \\
\text { user on the } \\
\text { filesystem } \\
\text { containing this } \\
\text { object - this should } \\
\text { be the smallest } \\
\text { relevant limit. }\end{array}$ \\
\hline space_free & 43 & uint 64 & $\mathrm{READ}$ & $\begin{array}{l}\text { Free disk space in } \\
\text { bytes on the } \\
\text { filesystem } \\
\text { containing this } \\
\text { object - this should }\end{array}$ \\
\hline
\end{tabular}




\begin{tabular}{|c|c|c|c|c|}
\hline & & & & $\begin{array}{l}\text { be the smallest } \\
\text { relevant limit. }\end{array}$ \\
\hline space_total & 44 & uint 64 & READ & $\begin{array}{l}\text { Total disk space in } \\
\text { bytes on the } \\
\text { filesystem } \\
\text { containing this } \\
\text { object. }\end{array}$ \\
\hline space_used & 45 & uint 64 & READ & $\begin{array}{l}\text { Number of filesystem } \\
\text { bytes allocated to } \\
\text { this object. }\end{array}$ \\
\hline system & 46 & bool & $R / W$ & $\begin{array}{l}\text { True, if this file } \\
\text { is a "system" file } \\
\text { with respect to the } \\
\text { Windows API. }\end{array}$ \\
\hline time_access & 47 & nfstime 4 & READ & $\begin{array}{l}\text { The time of last } \\
\text { access to the object } \\
\text { by a read that was } \\
\text { satisfied by the } \\
\text { server. }\end{array}$ \\
\hline time_access_set & 48 & settime 4 & WRITE & $\begin{array}{l}\text { Set the time of last } \\
\text { access to the } \\
\text { object. SETATTR } \\
\text { use only. }\end{array}$ \\
\hline time_backup & 49 & nfstime 4 & $\mathrm{R} / \mathrm{W}$ & $\begin{array}{l}\text { The time of last } \\
\text { backup of the } \\
\text { object. }\end{array}$ \\
\hline time_create & 50 & nfstime 4 & $\mathrm{R} / \mathrm{W}$ & $\begin{array}{l}\text { The time of creation } \\
\text { of the object. This } \\
\text { attribute does not } \\
\text { have any relation to } \\
\text { the traditional UNIX } \\
\text { file attribute } \\
\text { "ctime" or "change } \\
\text { time". }\end{array}$ \\
\hline time_delta & 51 & nfstime4 & READ & $\begin{array}{l}\text { Smallest useful } \\
\text { server time } \\
\text { granularity. }\end{array}$ \\
\hline
\end{tabular}




\begin{tabular}{|c|c|c|c|c|}
\hline time_metadata & 52 & nfstime 4 & READ & $\begin{array}{l}\text { The time of last } \\
\text { meta-data } \\
\text { modification of the } \\
\text { object. }\end{array}$ \\
\hline time_modify & 53 & nfstime4 & READ & $\begin{array}{l}\text { The time of last } \\
\text { modification to the } \\
\text { object. }\end{array}$ \\
\hline time_modify_set & 54 & settime 4 & WRITE & $\begin{array}{l}\text { Set the time of last } \\
\text { modification to the } \\
\text { object. SETATTR use } \\
\text { only. }\end{array}$ \\
\hline mounted_on_fileid & 55 & uint 64 & READ & $\begin{array}{l}\text { Like fileid, but if } \\
\text { the target } \\
\text { filehandle is the } \\
\text { root of a filesystem } \\
\text { return the fileid of } \\
\text { the underlying } \\
\text { directory. }\end{array}$ \\
\hline
\end{tabular}

\subsection{Time Access}

As defined above, the time_access attribute represents the time of last access to the object by a read that was satisfied by the server. The notion of what is an "access" depends on server's operating environment and/or the server's filesystem semantics. For example, for servers obeying POSIX semantics, time_access would be updated only by the READLINK, READ, and READDIR operations and not any of the operations that modify the content of the object. Of course, setting the corresponding time_access_set attribute is another way to modify the time_access attribute.

Whenever the file object resides on a writable filesystem, the server should make best efforts to record time_access into stable storage. However, to mitigate the performance effects of doing so, and most especially whenever the server is satisfying the read of the object's content from its cache, the server MAY cache access time updates and lazily write them to stable storage. It is also acceptable to give administrators of the server the option to disable time_access updates. 


\subsection{Interpreting owner and owner_group}

The recommended attributes "owner" and "owner_group" (and also users and groups within the "acl" attribute) are represented in terms of a UTF-8 string. To avoid a representation that is tied to a particular underlying implementation at the client or server, the use of the UTF-8 string has been chosen. Note that section 6.1 of [RFC2624] provides additional rationale. It is expected that the client and server will have their own local representation of owner and owner_group that is used for local storage or presentation to the end user. Therefore, it is expected that when these attributes are transferred between the client and server that the local representation is translated to a syntax of the form "user@dns_domain". This will allow for a client and server that do not use the same local representation the ability to translate to a common syntax that can be interpreted by both.

Similarly, security principals may be represented in different ways by different security mechanisms. Servers normally translate these representations into a common format, generally that used by local storage, to serve as a means of identifying the users corresponding to these security principals. When these local identifiers are translated to the form of the owner attribute, associated with files created by such principals they identify, in a common format, the users associated with each corresponding set of security principals.

The translation used to interpret owner and group strings is not specified as part of the protocol. This allows various solutions to be employed. For example, a local translation table may be consulted that maps between a numeric id to the userddns_domain syntax. A name service may also be used to accomplish the translation. A server may provide a more general service, not limited by any particular translation (which would only translate a limited set of possible strings) by storing the owner and owner_group attributes in local storage without any translation or it may augment a translation method by storing the entire string for attributes for which no translation is available while using the local representation for those cases in which a translation is available.

Servers that do not provide support for all possible values of the owner and owner_group attributes, should return an error (NFS4ERR_BADOWNER) when a string is presented that has no translation, as the value to be set for a SETATTR of the owner, owner_group, or acl attributes. When a server does accept an owner or owner_group value as valid on a SETATTR (and similarly for the owner and group strings in an acl), it is promising to return that same string when a corresponding GETATTR is done. Configuration changes and ill-constructed name translations (those that contain 
aliasing) may make that promise impossible to honor. Servers should make appropriate efforts to avoid a situation in which these attributes have their values changed when no real change to ownership has occurred.

The "dns_domain" portion of the owner string is meant to be a DNS domain name. For example, usereietf.org. Servers should accept as valid a set of users for at least one domain. A server may treat other domains as having no valid translations. A more general service is provided when a server is capable of accepting users for multiple domains, or for all domains, subject to security constraints.

In the case where there is no translation available to the client or server, the attribute value must be constructed without the "a". Therefore, the absence of the a from the owner or owner_group attribute signifies that no translation was available at the sender and that the receiver of the attribute should not use that string as a basis for translation into its own internal format. Even though the attribute value can not be translated, it may still be useful. In the case of a client, the attribute string may be used for local display of ownership.

To provide a greater degree of compatibility with previous versions of NFS (i.e., v2 and v3), which identified users and groups by 32-bit unsigned uid's and gid's, owner and group strings that consist of decimal numeric values with no leading zeros can be given a special interpretation by clients and servers which choose to provide such support. The receiver may treat such a user or group string as representing the same user as would be represented by a v2/v3 uid or gid having the corresponding numeric value. A server is not obligated to accept such a string, but may return an NFS4ERR_BADOWNER instead. To avoid this mechanism being used to subvert user and group translation, so that a client might pass all of the owners and groups in numeric form, a server SHOULD return an NFS4ERR_BADOWNER error when there is a valid translation for the user or owner designated in this way. In that case, the client must use the appropriate namedomain string and not the special form for compatibility.

The owner string "nobody" may be used to designate an anonymous user, which will be associated with a file created by a security principal that cannot be mapped through normal means to the owner attribute. 


\subsection{Character Case Attributes}

With respect to the case_insensitive and case_preserving attributes, each UCS-4 character (which UTF-8 encodes) has a "long descriptive name" [RFC1345] which may or may not included the word "CAPITAL" or "SMALL". The presence of SMALL or CAPITAL allows an NFS server to implement unambiguous and efficient table driven mappings for case insensitive comparisons, and non-case-preserving storage. For general character handling and internationalization issues, see the section "Internationalization".

\subsection{Quota Attributes}

For the attributes related to filesystem quotas, the following definitions apply:

quota_avail_soft

The value in bytes which represents the amount of additional disk space that can be allocated to this file or directory before the user may reasonably be warned. It is understood that this space may be consumed by allocations to other files or directories though there is a rule as to which other files or directories.

quota_avail_hard

The value in bytes which represent the amount of additional disk space beyond the current allocation that can be allocated to this file or directory before further allocations will be refused. It is understood that this space may be consumed by allocations to other files or directories.

quota_used

The value in bytes which represent the amount of disc space used by this file or directory and possibly a number of other similar files or directories, where the set of "similar" meets at least the criterion that allocating space to any file or directory in the set will reduce the "quota_avail_hard" of every other file or directory in the set.

Note that there may be a number of distinct but overlapping sets of files or directories for which a quota_used value is maintained (e.g., "all files with a given owner", "all files with a given group owner", etc.) .

The server is at liberty to choose any of those sets but should do so in a repeatable way. The rule may be configured perfilesystem or may be "choose the set with the smallest quota". 


\subsection{Access Control Lists}

The NFS version 4 ACL attribute is an array of access control entries (ACE). Although, the client can read and write the ACL attribute, the NFSv4 model is the server does all access control based on the server's interpretation of the ACL. If at any point the client wants to check access without issuing an operation that modifies or reads data or metadata, the client can use the OPEN and ACCESS operations to do so. There are various access control entry types, as defined in the section "ACE type". The server is able to communicate which $\mathrm{ACE}$ types are supported by returning the appropriate value within the aclsupport attribute. Each ACE covers one or more operations on a file or directory as described in the Section "ACE Access Mask". It may also contain one or more flags that modify the semantics of the $\mathrm{ACE}$ as defined in the section "ACE flag".

The NFS ACE attribute is defined as follows:

\begin{tabular}{|c|c|c|}
\hline typedef & $=$ uint32_t & acetype 4 ; \\
\hline typedef & $=$ uint 32_t & aceflag 4 ; \\
\hline typedef & $=$ uint 32_t & acemask 4 ; \\
\hline struct & nfsace 4\{ & \\
\hline & acetype 4 & type; \\
\hline & aceflag 4 & flag; \\
\hline & $\begin{array}{l}\text { acemask } 4 \\
\text { utf8str_mixed }\end{array}$ & $\begin{array}{l}\text { access_mask; } \\
\text { who; }\end{array}$ \\
\hline
\end{tabular}

To determine if a request succeeds, each nfsace4 entry is processed in order by the server. Only ACEs which have a "who" that matches the requester are considered. Each ACE is processed until all of the bits of the requester's access have been ALLOWED. Once a bit (see below) has been ALLOWED by an ACCESS_ALLOWED_ACE, it is no longer considered in the processing of later ACEs. If an ACCESS_DENIED_ACE is encountered where the requester's access still has unALLOWED bits in common with the "access_mask" of the ACE, the request is denied. However, unlike the ALLOWED and DENIED ACE types, the ALARM and AUDIT ACE types do not affect a requester's access, and instead are for triggering events as a result of a requester's access attempt.

Therefore, all AUDIT and ALARM ACEs are processed until end of the ACL. When the ACL is fully processed, if there are bits in requester's mask that have not been considered whether the server allows or denies the access is undefined. If there is a mode attribute on the file, then this cannot happen, since the mode's MODE4_*OTH bits will map to EVERYONE@ ACEs that unambiguously specify the requester's access. 
The NFS version $4 \mathrm{ACL}$ model is quite rich. Some server platforms may provide access control functionality that goes beyond the UNIX-style mode attribute, but which is not as rich as the NFS ACL model. So that users can take advantage of this more limited functionality, the server may indicate that it supports ACLs as long as it follows the guidelines for mapping between its ACL model and the NFS version 4 ACL model.

The situation is complicated by the fact that a server may have multiple modules that enforce ACLs. For example, the enforcement for NFS version 4 access may be different from the enforcement for local access, and both may be different from the enforcement for access through other protocols such as SMB. So it may be useful for a server to accept an ACL even if not all of its modules are able to support it.

The guiding principle in all cases is that the server must not accept ACLs that appear to make the file more secure than it really is.

5.11.1. ACE type

Type Description

\begin{tabular}{ll}
\hline ALLOW & $\begin{array}{l}\text { Explicitly grants the access defined in } \\
\text { acemask4 to the file or directory. }\end{array}$ \\
DENY & $\begin{array}{l}\text { Explicitly denies the access defined in } \\
\text { acemask4 to the file or directory. }\end{array}$ \\
AUDIT & LOG (system dependent) any access \\
& attempt to a file or directory which \\
& uses any of the access methods specified \\
& in acemask 4
\end{tabular}

ALARM Generate a system ALARM (system dependent) when any access attempt is made to a file or directory for the access methods specified in acemask 4.

A server need not support all of the above ACE types. The bitmask constants used to represent the above definitions within the

aclsupport attribute are as follows:

const ACL4_SUPPORT_ALLOW_ACL = 0x00000001;

const ACL4_SUPPORT_DENY_ACL $=0 \times 00000002$;

const ACL4_SUPPORT_AUDIT_ACL $=0 \times 00000004$;

const ACL4_SUPPORT_ALARM_ACL $=0 \times 00000008$; 
The semantics of the "type" field follow the descriptions provided above.

The constants used for the type field (acetype4) are as follows:

$$
\begin{array}{lll}
\text { const ACE4_ACCESS_ALLOWED_ACE_TYPE } & =0 \times 00000000 ; \\
\text { const ACE4_ACCESS_DENIED_ACE_TYPE } & =0 \times 00000001 ; \\
\text { const ACE4_SYSTEM_AUDIT_ACE_TYPE } & =0 \times 00000002 ; \\
\text { const ACE4_SYSTEM_ALARM_ACE_TYPE } & =0 \times 00000003 ;
\end{array}
$$

Clients should not attempt to set an ACE unless the server claims support for that $A C E$ type. If the server receives a request to set an $\mathrm{ACE}$ that it cannot store, it MUST reject the request with NFS4ERR_ATTRNOTSUPP. If the server receives a request to set an ACE that it can store but cannot enforce, the server SHould reject the request with NFS4ERR_ATTRNOTSUPP.

Example: suppose a server can enforce NFS ACLs for NFS access but cannot enforce ACLs for local access. If arbitrary processes can run on the server, then the server SHOULD NOT indicate ACL support. On the other hand, if only trusted administrative programs run locally, then the server may indicate ACL support.

\begin{tabular}{|c|c|}
\hline READ_DATA & Permission to read the data of the file \\
\hline LIST_DIRECTORY & $\begin{array}{l}\text { Permission to list the contents of a } \\
\text { directory }\end{array}$ \\
\hline WRITE_DATA & Permission to modify the file's data \\
\hline ADD_FILE & $\begin{array}{l}\text { Permission to add a new file to a } \\
\text { directory }\end{array}$ \\
\hline APPEND_DATA & Permission to append data to a file \\
\hline ADD_SUBDIRECTORY & $\begin{array}{l}\text { Permission to create a subdirectory to a } \\
\text { directory }\end{array}$ \\
\hline READ_NAMED_ATTRS & $\begin{array}{l}\text { Permission to read the named attributes } \\
\text { of a file }\end{array}$ \\
\hline WRITE_NAMED_ATTRS & $\begin{array}{l}\text { Permission to write the named attributes } \\
\text { of a file }\end{array}$ \\
\hline EXECUTE & Permission to execute a file \\
\hline DELETE_CHILD & $\begin{array}{l}\text { Permission to delete a file or directory } \\
\text { within a directory }\end{array}$ \\
\hline READ_ATTRIBUTES & $\begin{array}{l}\text { The ability to read basic attributes } \\
\text { (non-acls) of a file }\end{array}$ \\
\hline WRITE_ATTRIBUTES & Permission to change basic attributes \\
\hline
\end{tabular}

5.11.2. ACE Access Mask

The access_mask field contains values based on the following:

Access Description 


\begin{tabular}{|c|c|c|c|}
\hline \\
\hline \multicolumn{4}{|c|}{ (non-acls) of a file } \\
\hline \multicolumn{4}{|c|}{ READ_ACL } \\
\hline \multicolumn{4}{|c|}{ WRITE_ACL } \\
\hline \multicolumn{4}{|c|}{ WRITE_OWNER } \\
\hline \multicolumn{4}{|c|}{ SYNCHRONIZE } \\
\hline \multicolumn{4}{|c|}{ The bitmask constants used for the access mas } \\
\hline \multicolumn{2}{|c|}{ const ACE4_READ_DATA } & $=$ & $0 \times 00000001$ \\
\hline \multicolumn{2}{|c|}{ const ACE4_LIST_DIRECTORY } & $=$ & 0 \\
\hline \multicolumn{2}{|c|}{ const ACE4_WRITE_DATA } & $=$ & $0 \times 000$ \\
\hline \multicolumn{2}{|c|}{ const ACE4_ADD_FILE } & $=$ & $0 \times 0000$ \\
\hline \multicolumn{2}{|c|}{ const ACE4_APPEND_DATA } & $=$ & $0 \times 0000$ \\
\hline \multicolumn{2}{|c|}{ const ACE4_ADD_SUBDIRECTORY } & $=$ & $0 \times 0000$ \\
\hline \multicolumn{2}{|c|}{ const ACE4_READ_NAMED_ATTRS } & $=$ & $0 \times 0000$ \\
\hline \multicolumn{2}{|c|}{ const ACE4_WRITE_NAMED_ATTRS } & $=$ & $0 \times 0000$ \\
\hline \multicolumn{2}{|c|}{ const ACE4_EXECUTE } & $=$ & $0 \times 0000$ \\
\hline \multicolumn{2}{|c|}{ const ACE4_DELETE_CHILD } & $=$ & $0 \times 0000$ \\
\hline \multicolumn{2}{|c|}{ const ACE4_READ_ATTRIBUTES } & $=$ & $0 \times 0000008$ \\
\hline \multicolumn{2}{|c|}{ const ACE4_WRITE_ATTRIBUTES } & $=$ & $0 \times 0000$ \\
\hline \multicolumn{2}{|c|}{ const ACE4_DELETE } & $=$ & $0 \times 00010000$ \\
\hline \multicolumn{2}{|c|}{ const ACE4_READ_ACL } & $=$ & $0 \times 00020000$ \\
\hline \multicolumn{2}{|c|}{ const ACE4_WRITE_ACL } & $=$ & $0 \times 00040000$ \\
\hline \multicolumn{2}{|c|}{ const ACE4_WRITE_OWNER } & $=$ & $0 \times 000$ \\
\hline \multicolumn{2}{|c|}{ const ACE4_SYNCHRONIZE } & $=$ & $0 \times 0010$ \\
\hline
\end{tabular}

Server implementations need not provide the granularity of control that is implied by this list of masks. For example, PosIX-based systems might not distinguish APPEND_DATA (the ability to append to a file) from WRITE_DATA (the ability to modify existing contents); both masks would be tied to a single "write" permission. When such a server returns attributes to the client, it would show both APPEND_DATA and WRITE_DATA if and only if the write permission is enabled.

If a server receives a SETATTR request that it cannot accurately implement, it should error in the direction of more restricted access. For example, suppose a server cannot distinguish overwriting data from appending new data, as described in the previous paragraph. If a client submits an ACE where APPEND_DATA is set but WRITE_DATA is not (or vice versa), the server should reject the request with NFS4ERR_ATTRNOTSUPP. Nonetheless, if the ACE has type DENY, the server may silently turn on the other bit, so that both APPEND_DATA and WRITE_DATA are denied. 


\subsubsection{ACE flag}

The "flag" field contains values based on the following descriptions.

ACE4_FILE_INHERIT_ACE

Can be placed on a directory and indicates that this ACE should be added to each new non-directory file created.

ACE4_DIRECTORY_INHERIT_ACE

Can be placed on a directory and indicates that this ACE should be added to each new directory created.

ACE4_INHERIT_ONLY_ACE

Can be placed on a directory but does not apply to the directory, only to newly created files/directories as specified by the above two flags.

ACE4_NO_PROPAGATE_INHERIT_ACE

Can be placed on a directory. Normally when a new directory is created and an $\mathrm{ACE}$ exists on the parent directory which is marked ACL4_DIRECTORY_INHERIT_ACE, two ACEs are placed on the new directory. One for the directory itself and one which is an inheritable ACE for newly created directories. This flag tells the server to not place an $\mathrm{ACE}$ on the newly created directory which is inheritable by subdirectories of the created directory.

ACE4_SUCCESSFUL_ACCESS_ACE_FLAG

ACL4_FAILED_ACCESS_ACE_FLAG

The ACE4_SUCCESSFUL_ACCESS_ACE_FLAG (SUCCESS) and

ACE4_FAILED_ACCESS_ACE_FLAG (FAILED) flag bits relate only to ACE4_SYSTEM_AUDIT_ACE_TYPE (AUDIT) and ACE4_SYSTEM_ALARM_ACE_TYPE

(ALARM) ACE types. If during the processing of the file's ACL, the server encounters an AUDIT or ALARM ACE that matches the principal attempting the OPEN, the server notes that fact, and the presence, if any, of the SUCCESS and FAILED flags encountered in the AUDIT or ALARM ACE. Once the server completes the ACL processing, and the share reservation processing, and the OPEN call, it then notes if the OPEN succeeded or failed. If the OPEN succeeded, and if the SUCCESS flag was set for a matching AUDIT or ALARM, then the appropriate AUDIT or ALARM event occurs. If the OPEN failed, and if the FAILED flag was set for the matching AUDIT or ALARM, then the appropriate AUDIT or ALARM event occurs. Clearly either or both of the SUCCESS or FAILED can be set, but if neither is set, the AUDIT or ALARM ACE is not useful. 
The previously described processing applies to that of the ACCESS operation as well. The difference being that "success" or "failure" does not mean whether ACCESS returns NFS4_OK or not. Success means whether ACCESS returns all requested and supported bits. Failure means whether ACCESS failed to return a bit that was requested and supported.

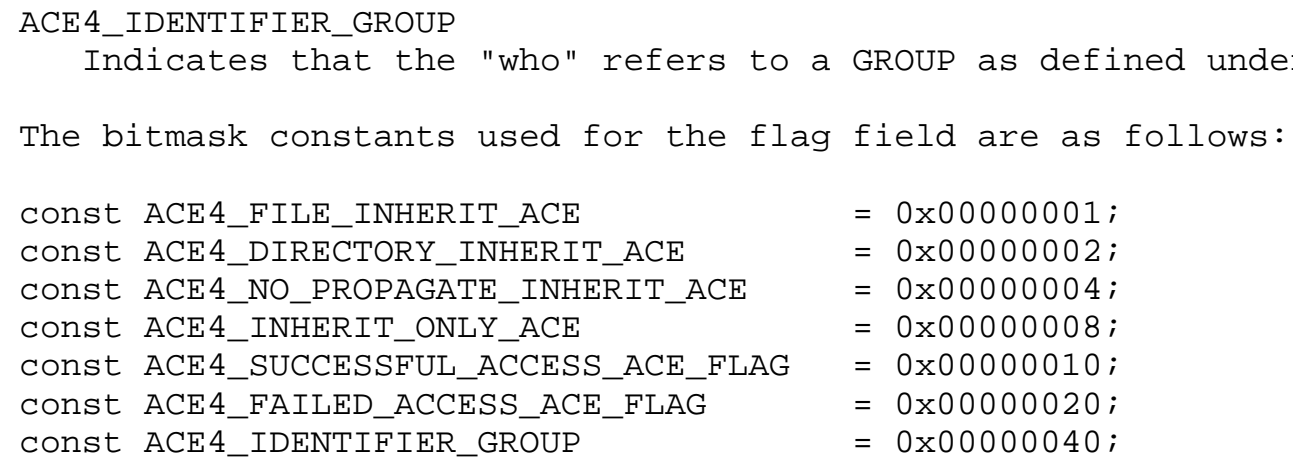

A server need not support any of these flags. If the server supports flags that are similar to, but not exactly the same as, these flags, the implementation may define a mapping between the protocol-defined flags and the implementation-defined flags. Again, the guiding principle is that the file not appear to be more secure than it really is.

For example, suppose a client tries to set an ACE with ACE4_FILE_INHERIT_ACE set but not ACE4_DIRECTORY_INHERIT_ACE. If the server does not support any form of ACL inheritance, the server should reject the request with NFS4ERR_ATTRNOTSUPP. If the server supports a single "inherit ACE" flag that applies to both files and directories, the server may reject the request (i.e., requiring the client to set both the file and directory inheritance flags). The server may also accept the request and silently turn on the ACE4_DIRECTORY_INHERIT_ACE flag.

\subsubsection{ACE who}

There are several special identifiers ("who") which need to be understood universally, rather than in the context of a particular DNS domain. Some of these identifiers cannot be understood when an NFS client accesses the server, but have meaning when a local process accesses the file. The ability to display and modify these permissions is permitted over NFS, even if none of the access methods on the server understands the identifiers. 


\begin{tabular}{ll} 
Who & Description \\
\hline "OWNER" & The owner of the file. \\
"GROUP" & The group associated with the file. \\
"EVERYONE" & The world. \\
"INTERACTIVE" & Accessed from an interactive terminal. \\
"NETWORK" & Accessed via the network. \\
"DIALUP" & Accessed as a dialup user to the server. \\
"BATCH" & Accessed from a batch job. \\
"ANONYMOUS" & Accessed without any authentication. \\
"AUTHENTICATED" & Any authenticated user (opposite of \\
"SERVICE" & ANONYMOUS) \\
\end{tabular}

To avoid conflict, these special identifiers are distinguish by an appended "Q" and should appear in the form "xxxxe" (note: no domain name after the "("). For example: ANONYMOUSe.

\subsubsection{Mode Attribute}

The NFS version 4 mode attribute is based on the UNIX mode bits. The following bits are defined:

\begin{tabular}{|c|c|c|c|}
\hline & & & /* \\
\hline st & ODE4_SGID & $=0 x$ & /* set group id on execution */ \\
\hline & MODE4_SVTX & $=0 x$ & /* save text even after use */ \\
\hline & 4_RUSR & $=$ & /* read permission: owner */ \\
\hline & MODE 4_WUSR & $=0$ & /* write permission: owner */ \\
\hline & $R$ & $=$ & xecute permission: owner */ \\
\hline & MODE 4_RGRP & $=0$ & / read permission: group */ \\
\hline & MODE 4_WGRP & $=0$ & /* write permission: group */ \\
\hline & MODE4_XGRP & $=0$ & / execute permission: group \\
\hline & MODE4_ROTH & $=0$ & /* read permission: other */ \\
\hline & MOD & $=0$ & write permission: other */ \\
\hline & $\mathrm{TH}$ & $=0$ & xecute permission: other \\
\hline
\end{tabular}

Bits MODE4_RUSR, MODE4_WUSR, and MODE4_XUSR apply to the principal identified in the owner attribute. Bits MODE4_RGRP, MODE4_WGRP, and

MODE4_XGRP apply to the principals identified in the owner_group attribute. Bits MODE4_ROTH, MODE4_WOTH, MODE4_XOTH apply to any principal that does not match that in the owner group, and does not have a group matching that of the owner_group attribute.

The remaining bits are not defined by this protocol and MUST NOT be used. The minor version mechanism must be used to define further bit usage. 
Note that in UNIX, if a file has the MODE4_SGID bit set and no MODE4_XGRP bit set, then READ and WRITE must use mandatory file locking.

\subsubsection{Mode and ACL Attribute}

The server that supports both mode and ACL must take care to synchronize the MODE4_*USR, MODE4_*GRP, and MODE4_*OTH bits with the ACEs which have respective who fields of "OWNERe", "GROUPC", and "EVERYONEQ" so that the client can see semantically equivalent access permissions exist whether the client asks for owner, owner_group and mode attributes, or for just the ACL.

Because the mode attribute includes bits (e.g., MODE4_SVTX) that have nothing to do with ACL semantics, it is permitted for clients to specify both the ACL attribute and mode in the same SETATTR operation. However, because there is no prescribed order for processing the attributes in a SETATTR, the client must ensure that ACL attribute, if specified without mode, would produce the desired mode bits, and conversely, the mode attribute if specified without ACL, would produce the desired "OWNERQ", "GROUPC", and "EVERYONEQ" ACES.

\subsection{7. mounted_on_fileid}

UNIX-based operating environments connect a filesystem into the namespace by connecting (mounting) the filesystem onto the existing file object (the mount point, usually a directory) of an existing filesystem. When the mount point's parent directory is read via an API like readdir(), the return results are directory entries, each with a component name and a fileid. The fileid of the mount point's directory entry will be different from the fileid that the stat() system call returns. The stat() system call is returning the fileid of the root of the mounted filesystem, whereas readdir() is returning the fileid stat() would have returned before any filesystems were mounted on the mount point.

Unlike NFS version 3, NFS version 4 allows a client's LOOKUP request to cross other filesystems. The client detects the filesystem crossing whenever the filehandle argument of LOOKUP has an fsid attribute different from that of the filehandle returned by LOOKUP. A UNIX-based client will consider this a "mount point crossing". UNIX has a legacy scheme for allowing a process to determine its current working directory. This relies on readdir() of a mount point's parent and stat() of the mount point returning fileids as previously described. The mounted_on_fileid attribute corresponds to the fileid that readdir() would have returned as described previously. 
While the NFS version 4 client could simply fabricate a fileid corresponding to what mounted_on_fileid provides (and if the server does not support mounted_on_fileid, the client has no choice), there is a risk that the client will generate a fileid that conflicts with one that is already assigned to another object in the filesystem. Instead, if the server can provide the mounted_on_fileid, the potential for client operational problems in this area is eliminated.

If the server detects that there is no mounted point at the target file object, then the value for mounted_on_fileid that it returns is the same as that of the fileid attribute.

The mounted_on_fileid attribute is RECOMMENDED, so the server SHOULD provide it if possible, and for a UNIX-based server, this is straightforward. Usually, mounted_on_fileid will be requested during a READDIR operation, in which case it is trivial (at least for UNIXbased servers) to return mounted_on_fileid since it is equal to the fileid of a directory entry returned by readdir(). If mounted_on_fileid is requested in a GETATTR operation, the server should obey an invariant that has it returning a value that is equal to the file object's entry in the object's parent directory, i.e., what readdir() would have returned. Some operating environments allow a series of two or more filesystems to be mounted onto a single mount point. In this case, for the server to obey the aforementioned invariant, it will need to find the base mount point, and not the intermediate mount points.

6. Filesystem Migration and Replication

With the use of the recommended attribute "fs_locations", the NFS version 4 server has a method of providing filesystem migration or replication services. For the purposes of migration and replication, a filesystem will be defined as all files that share a given fsid (both major and minor values are the same).

The fs_locations attribute provides a list of filesystem locations. These locations are specified by providing the server name (either DNS domain or IP address) and the path name representing the root of the filesystem. Depending on the type of service being provided, the list will provide a new location or a set of alternate locations for the filesystem. The client will use this information to redirect its requests to the new server.

\subsection{Replication}

It is expected that filesystem replication will be used in the case of read-only data. Typically, the filesystem will be replicated on two or more servers. The fs_locations attribute will provide the 
list of these locations to the client. On first access of the filesystem, the client should obtain the value of the fs_locations attribute. If, in the future, the client finds the server unresponsive, the client may attempt to use another server specified by fs_locations.

If applicable, the client must take the appropriate steps to recover valid filehandles from the new server. This is described in more detail in the following sections.

\subsection{Migration}

Filesystem migration is used to move a filesystem from one server to another. Migration is typically used for a filesystem that is writable and has a single copy. The expected use of migration is for load balancing or general resource reallocation. The protocol does not specify how the filesystem will be moved between servers. This server-to-server transfer mechanism is left to the server implementor. However, the method used to communicate the migration event between client and server is specified here.

Once the servers participating in the migration have completed the move of the filesystem, the error NFS4ERR_MOVED will be returned for subsequent requests received by the original server. The NFS4ERR_MOVED error is returned for all operations except PUTFH and GETATTR. Upon receiving the NFS4ERR_MOVED error, the client will obtain the value of the fs_locations attribute. The client will then use the contents of the attribute to redirect its requests to the specified server. To facilitate the use of GETATTR, operations such as PUTFH must also be accepted by the server for the migrated file system's filehandles. Note that if the server returns NFS4ERR_MOVED, the server MUST support the fs_locations attribute.

If the client requests more attributes than just fs_locations, the server may return fs_locations only. This is to be expected since the server has migrated the filesystem and may not have a method of obtaining additional attribute data.

The server implementor needs to be careful in developing a migration solution. The server must consider all of the state information clients may have outstanding at the server. This includes but is not limited to locking/share state, delegation state, and asynchronous file writes which are represented by WRITE and COMMIT verifiers. The server should strive to minimize the impact on its clients during and after the migration process. 


\subsection{Interpretation of the fs_locations Attribute}

The fs_location attribute is structured in the following way:

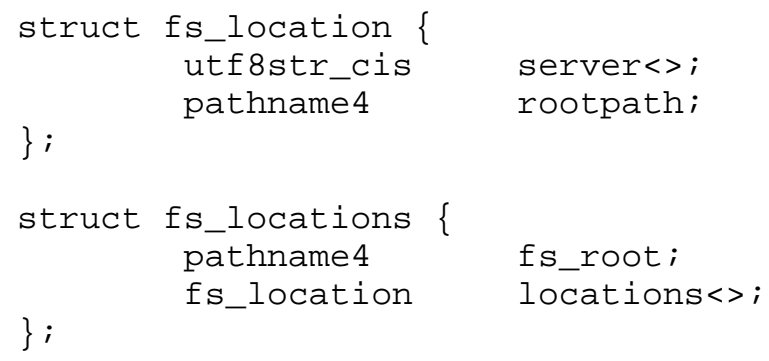

The fs_location struct is used to represent the location of a filesystem by providing a server name and the path to the root of the filesystem. For a multi-homed server or a set of servers that use the same rootpath, an array of server names may be provided. An entry in the server array is an UTF8 string and represents one of a traditional DNS host name, IPv4 address, or IPv6 address. It is not a requirement that all servers that share the same rootpath be listed in one fs_location struct. The array of server names is provided for convenience. Servers that share the same rootpath may also be listed in separate fs_location entries in the fs_locations attribute.

The fs_locations struct and attribute then contains an array of locations. Since the name space of each server may be constructed differently, the "fs_root" field is provided. The path represented by fs_root represents the location of the filesystem in the server's name space. Therefore, the fs_root path is only associated with the server from which the fs_locations attribute was obtained. The fs_root path is meant to aid the client in locating the filesystem at the various servers listed.

As an example, there is a replicated filesystem located at two servers (servA and servB). At servA the filesystem is located at path "/a/b/c". At servB the filesystem is located at path "/x/y/z". In this example the client accesses the filesystem first at servA with a multi-component lookup path of "/a/b/c/d". Since the client used a multi-component lookup to obtain the filehandle at "/a/b/c/d", it is unaware that the filesystem's root is located in servA's name space at "/a/b/c". When the client switches to servB, it will need to determine that the directory it first referenced at servA is now represented by the path "/x/y/z/d" on servB. To facilitate this, the fs_locations attribute provided by servA would have a fs_root value of $" / \mathrm{a} / \mathrm{b} / \mathrm{c} "$ and two entries in fs_location. One entry in fs_location will be for itself (servA) and the other will be for servB with a 
path of $" / x / y / z "$. With this information, the client is able to substitute "/x/y/z" for the "/a/b/c" at the beginning of its access path and construct "/x/y/z/d" to use for the new server.

See the section "Security Considerations" for a discussion on the recommendations for the security flavor to be used by any GETATTR operation that requests the "fs_locations" attribute.

\subsection{Filehandle Recovery for Migration or Replication}

Filehandles for filesystems that are replicated or migrated generally have the same semantics as for filesystems that are not replicated or migrated. For example, if a filesystem has persistent filehandles and it is migrated to another server, the filehandle values for the filesystem will be valid at the new server.

For volatile filehandles, the servers involved likely do not have a mechanism to transfer filehandle format and content between themselves. Therefore, a server may have difficulty in determining if a volatile filehandle from an old server should return an error of NFS4ERR_FHEXPIRED. Therefore, the client is informed, with the use of the fh_expire_type attribute, whether volatile filehandles will expire at the migration or replication event. If the bit FH4_VOL_MIGRATION is set in the fh_expire_type attribute, the client must treat the volatile filehandle as if the server had returned the NFS4ERR_FHEXPIRED error. At the migration or replication event in the presence of the FH4_VOL_MIGRATION bit, the client will not present the original or old volatile filehandle to the new server. The client will start its communication with the new server by recovering its filehandles using the saved file names.

7. NFS Server Name Space

\subsection{Server Exports}

On a UNIX server the name space describes all the files reachable by pathnames under the root directory or "/". On a Windows NT server the name space constitutes all the files on disks named by mapped disk letters. NFS server administrators rarely make the entire server's filesystem name space available to NFS clients. More often portions of the name space are made available via an "export" feature. In previous versions of the NFS protocol, the root filehandle for each export is obtained through the MOUNT protocol; the client sends a string that identifies the export of name space and the server returns the root filehandle for it. The MOUNT protocol supports an EXPORTS procedure that will enumerate the server's exports. 


\subsection{Browsing Exports}

The NFS version 4 protocol provides a root filehandle that clients can use to obtain filehandles for these exports via a multi-component LOOKUP. A common user experience is to use a graphical user interface (perhaps a file "Open" dialog window) to find a file via progressive browsing through a directory tree. The client must be able to move from one export to another export via single-component, progressive LOOKUP operations.

This style of browsing is not well supported by the NFS version 2 and 3 protocols. The client expects all LookUP operations to remain within a single server filesystem. For example, the device attribute will not change. This prevents a client from taking name space paths that span exports.

An automounter on the client can obtain a snapshot of the server's name space using the EXPORTS procedure of the MOUNT protocol. If it understands the server's pathname syntax, it can create an image of the server's name space on the client. The parts of the name space that are not exported by the server are filled in with a "pseudo filesystem" that allows the user to browse from one mounted filesystem to another. There is a drawback to this representation of the server's name space on the client: it is static. If the server administrator adds a new export the client will be unaware of it.

\subsection{Server Pseudo Filesystem}

NFS version 4 servers avoid this name space inconsistency by presenting all the exports within the framework of a single server name space. An NFS version 4 client uses LOOKUP and READDIR operations to browse seamlessly from one export to another. Portions of the server name space that are not exported are bridged via a "pseudo filesystem" that provides a view of exported directories only. A pseudo filesystem has a unique fsid and behaves like a normal, read only filesystem.

Based on the construction of the server's name space, it is possible that multiple pseudo filesystems may exist. For example,
/a pseudo filesystem
$/ \mathrm{a} / \mathrm{b} \quad$ real filesystem
$/ \mathrm{a} / \mathrm{b} / \mathrm{c} \quad$ pseudo filesystem
$/ \mathrm{a} / \mathrm{b} / \mathrm{c} / \mathrm{d}$ real filesystem

Each of the pseudo filesystems are considered separate entities and therefore will have a unique fsid. 


\subsection{Multiple Roots}

The DOS and Windows operating environments are sometimes described as having "multiple roots". Filesystems are commonly represented as disk letters. MacOS represents filesystems as top level names. NFS version 4 servers for these platforms can construct a pseudo file system above these root names so that disk letters or volume names are simply directory names in the pseudo root.

\subsection{Filehandle Volatility}

The nature of the server's pseudo filesystem is that it is a logical representation of filesystem(s) available from the server. Therefore, the pseudo filesystem is most likely constructed dynamically when the server is first instantiated. It is expected that the pseudo filesystem may not have an on disk counterpart from which persistent filehandles could be constructed. Even though it is preferable that the server provide persistent filehandles for the pseudo filesystem, the NFS client should expect that pseudo file system filehandles are volatile. This can be confirmed by checking the associated "fh_expire_type" attribute for those filehandles in question. If the filehandles are volatile, the NFS client must be prepared to recover a filehandle value (e.g., with a multi-component LOOKUP) when receiving an error of NFS4ERR_FHEXPIRED.

\subsection{Exported Root}

If the server's root filesystem is exported, one might conclude that a pseudo-filesystem is not needed. This would be wrong. Assume the following filesystems on a server:

$\begin{array}{lll}/ & \text { disk1 } & \text { (exported) } \\ / \mathrm{a} & \text { disk2 } & \text { (not exported) } \\ / \mathrm{a} / \mathrm{b} & \text { disk3 } & \text { (exported) }\end{array}$

Because disk2 is not exported, disk3 cannot be reached with simple LOOKUPs. The server must bridge the gap with a pseudo-filesystem.

\subsection{Mount Point Crossing}

The server filesystem environment may be constructed in such a way that one filesystem contains a directory which is 'covered' or mounted upon by a second filesystem. For example:

$$
\begin{array}{ll}
/ \mathrm{a} / \mathrm{b} & (\text { filesystem } 1) \\
/ \mathrm{a} / \mathrm{b} / \mathrm{c} / \mathrm{d} & (\text { filesystem 2) }
\end{array}
$$


The pseudo filesystem for this server may be constructed to look like:

$$
\begin{array}{ll}
/ & (\text { place holder/not exported) } \\
/ \mathrm{a} / \mathrm{b} & (\text { filesystem } 1) \\
/ \mathrm{a} / \mathrm{b} / \mathrm{c} / \mathrm{d} & (\text { filesystem } 2)
\end{array}
$$

It is the server's responsibility to present the pseudo filesystem that is complete to the client. If the client sends a lookup request for the path "/a/b/c/d", the server's response is the filehandle of the filesystem "/a/b/c/d". In previous versions of the NFS protocol, the server would respond with the filehandle of directory "/a/b/c/d" within the filesystem "/a/b".

The NFS client will be able to determine if it crosses a server mount point by a change in the value of the "fsid" attribute.

\subsection{Security Policy and Name space Presentation}

The application of the server's security policy needs to be carefully considered by the implementor. One may choose to limit the viewability of portions of the pseudo filesystem based on the server's perception of the client's ability to authenticate itself properly. However, with the support of multiple security mechanisms and the ability to negotiate the appropriate use of these mechanisms, the server is unable to properly determine if a client will be able to authenticate itself. If, based on its policies, the server chooses to limit the contents of the pseudo filesystem, the server may effectively hide filesystems from a client that may otherwise have legitimate access.

As suggested practice, the server should apply the security policy of a shared resource in the server's namespace to the components of the resource's ancestors. For example:

\section{/}

$/ \mathrm{a} / \mathrm{b}$

$/ \mathrm{a} / \mathrm{b} / \mathrm{c}$

The /a/b/c directory is a real filesystem and is the shared resource. The security policy for /a/b/c is Kerberos with integrity. The server should apply the same security policy to /, /a, and /a/b. This allows for the extension of the protection of the server's namespace to the ancestors of the real shared resource. 
For the case of the use of multiple, disjoint security mechanisms in the server's resources, the security for a particular object in the server's namespace should be the union of all security mechanisms of all direct descendants.

8. File Locking and Share Reservations

Integrating locking into the NFS protocol necessarily causes it to be stateful. With the inclusion of share reservations the protocol becomes substantially more dependent on state than the traditional combination of NFS and NLM [XNFS]. There are three components to making this state manageable:

- Clear division between client and server

- Ability to reliably detect inconsistency in state between client and server

- Simple and robust recovery mechanisms

In this model, the server owns the state information. The client communicates its view of this state to the server as needed. The client is also able to detect inconsistent state before modifying a file.

To support Win32 share reservations it is necessary to atomically OPEN or CREATE files. Having a separate share/unshare operation would not allow correct implementation of the Win32 openFile API. In order to correctly implement share semantics, the previous NFS protocol mechanisms used when a file is opened or created (LOOKUP, CREATE, ACCESS) need to be replaced. The NFS version 4 protocol has an OPEN operation that subsumes the NFS version 3 methodology of LOOKUP, CREATE, and ACCESS. However, because many operations require a filehandle, the traditional LOOKUP is preserved to map a file name to filehandle without establishing state on the server. The policy of granting access or modifying files is managed by the server based on the client's state. These mechanisms can implement policy ranging from advisory only locking to full mandatory locking.

\subsection{Locking}

It is assumed that manipulating a lock is rare when compared to $\mathrm{READ}$ and WRITE operations. It is also assumed that crashes and network partitions are relatively rare. Therefore it is important that the READ and WRITE operations have a lightweight mechanism to indicate if they possess a held lock. A lock request contains the heavyweight information required to establish a lock and uniquely define the lock owner. 
The following sections describe the transition from the heavy weight information to the eventual stateid used for most client and server locking and lease interactions.

\subsubsection{Client ID}

For each LOCK request, the client must identify itself to the server.

This is done in such a way as to allow for correct lock identification and crash recovery. A sequence of a SETCLIENTID operation followed by a SETCLIENTID_CONFIRM operation is required to establish the identification onto the server. Establishment of identification by a new incarnation of the client also has the effect of immediately breaking any leased state that a previous incarnation of the client might have had on the server, as opposed to forcing the new client incarnation to wait for the leases to expire. Breaking the lease state amounts to the server removing all lock, share reservation, and, where the server is not supporting the CLAIM_DELEGATE_PREV claim type, all delegation state associated with same client with the same identity. For discussion of delegation state recovery, see the section "Delegation Recovery".

Client identification is encapsulated in the following structure:

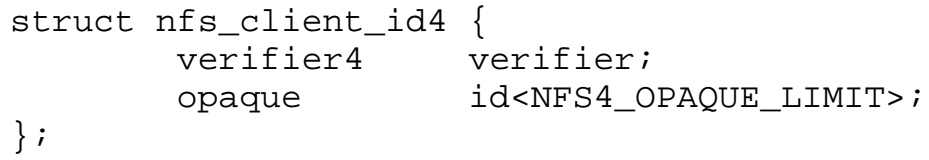

The first field, verifier is a client incarnation verifier that is used to detect client reboots. Only if the verifier is different from that which the server has previously recorded the client (as identified by the second field of the structure, id) does the server start the process of canceling the client's leased state.

The second field, id is a variable length string that uniquely defines the client.

There are several considerations for how the client generates the id string:

- The string should be unique so that multiple clients do not present the same string. The consequences of two clients presenting the same string range from one client getting an error to one client having its leased state abruptly and unexpectedly canceled. 
- The string should be selected so the subsequent incarnations (e.g., reboots) of the same client cause the client to present the same string. The implementor is cautioned against an approach that requires the string to be recorded in a local file because this precludes the use of the implementation in an environment where there is no local disk and all file access is from an NFS version 4 server.

- The string should be different for each server network address that the client accesses, rather than common to all server network addresses. The reason is that it may not be possible for the client to tell if the same server is listening on multiple network addresses. If the client issues SETCLIENTID with the same id string to each network address of such a server, the server will think it is the same client, and each successive SETCLIENTID will cause the server to begin the process of removing the client's previous leased state.

- The algorithm for generating the string should not assume that the client's network address won't change. This includes changes between client incarnations and even changes while the client is stilling running in its current incarnation. This means that if the client includes just the client's and server's network address in the id string, there is a real risk, after the client gives up the network address, that another client, using a similar algorithm for generating the id string, will generate a conflicting id string.

Given the above considerations, an example of a well generated id string is one that includes:

o The server's network address.

o The client's network address.

- For a user level NFS version 4 client, it should contain additional information to distinguish the client from other user level clients running on the same host, such as a process id or other unique sequence.

- Additional information that tends to be unique, such as one or more of:

- The client machine's serial number (for privacy reasons, it is best to perform some one way function on the serial number).

- A MAC address. 
- The timestamp of when the NFS version 4 software was first installed on the client (though this is subject to the previously mentioned caution about using information that is stored in a file, because the file might only be accessible over NFS version 4).

- A true random number. However since this number ought to be the same between client incarnations, this shares the same problem as that of the using the timestamp of the software installation.

As a security measure, the server MUST NOT cancel a client's leased state if the principal established the state for a given id string is not the same as the principal issuing the SETCLIENTID.

Note that SETCLIENTID and SETCLIENTID_CONFIRM has a secondary purpose of establishing the information the server needs to make callbacks to the client for purpose of supporting delegations. It is permitted to change this information via SETCLIENTID and SETCLIENTID_CONFIRM within the same incarnation of the client without removing the client's leased state.

Once a SETCLIENTID and SETCLIENTID_CONFIRM sequence has successfully completed, the client uses the shorthand client identifier, of type clientid4, instead of the longer and less compact nfs_client_id4 structure. This shorthand client identifier (a clientid) is assigned by the server and should be chosen so that it will not conflict with a clientid previously assigned by the server. This applies across server restarts or reboots. When a clientid is presented to a server and that clientid is not recognized, as would happen after a server reboot, the server will reject the request with the error NFS4ERR_STALE_CLIENTID. When this happens, the client must obtain a new clientid by use of the SETCLIENTID operation and then proceed to any other necessary recovery for the server reboot case (See the section "Server Failure and Recovery").

The client must also employ the SETCLIENTID operation when it receives a NFS4ERR_STALE_STATEID error using a stateid derived from its current clientid, since this also indicates a server reboot which has invalidated the existing clientid (see the next section "lock_owner and stateid Definition" for details).

See the detailed descriptions of SETCLIENTID and SETCLIENTID_CONFIRM for a complete specification of the operations. 


\subsubsection{Server Release of Clientid}

If the server determines that the client holds no associated state for its clientid, the server may choose to release the clientid. The server may make this choice for an inactive client so that resources are not consumed by those intermittently active clients. If the client contacts the server after this release, the server must ensure the client receives the appropriate error so that it will use the SETCLIENTID/SETCLIENTID_CONFIRM sequence to establish a new identity. It should be clear that the server must be very hesitant to release a clientid since the resulting work on the client to recover from such an event will be the same burden as if the server had failed and restarted. Typically a server would not release a clientid unless there had been no activity from that client for many minutes.

Note that if the id string in a SETCLIENTID request is properly constructed, and if the client takes care to use the same principal for each successive use of SETCLIENTID, then, barring an active denial of service attack, NFS4ERR_CLID_INUSE should never be returned.

However, client bugs, server bugs, or perhaps a deliberate change of the principal owner of the id string (such as the case of a client that changes security flavors, and under the new flavor, there is no mapping to the previous owner) will in rare cases result in NFS4ERR_CLID_INUSE.

In that event, when the server gets a SETCLIENTID for a client id that currently has no state, or it has state, but the lease has expired, rather than returning NFS4ERR_CLID_INUSE, the server MUST allow the SETCLIENTID, and confirm the new clientid if followed by the appropriate SETCLIENTID_CONFIRM.

\subsection{3. lock_owner and stateid Definition}

When requesting a lock, the client must present to the server the clientid and an identifier for the owner of the requested lock. These two fields are referred to as the lock_owner and the definition of those fields are:

- A clientid returned by the server as part of the client's use of the SETCLIENTID operation.

- A variable length opaque array used to uniquely define the owner of a lock managed by the client.

This may be a thread id, process id, or other unique value. 
When the server grants the lock, it responds with a unique stateid. The stateid is used as a shorthand reference to the lock_owner, since the server will be maintaining the correspondence between them.

The server is free to form the stateid in any manner that it chooses as long as it is able to recognize invalid and out-of-date stateids. This requirement includes those stateids generated by earlier instances of the server. From this, the client can be properly notified of a server restart. This notification will occur when the client presents a stateid to the server from a previous instantiation.

The server must be able to distinguish the following situations and return the error as specified:

- The stateid was generated by an earlier server instance (i.e., before a server reboot). The error NFS4ERR_STALE_STATEID should be returned.

- The stateid was generated by the current server instance but the stateid no longer designates the current locking state for the lockowner-file pair in question (i.e., one or more locking operations has occurred). The error NFS4ERR_OLD_STATEID should be returned.

This error condition will only occur when the client issues a locking request which changes a stateid while an I/O request that uses that stateid is outstanding.

- The stateid was generated by the current server instance but the stateid does not designate a locking state for any active lockowner-file pair. The error NFS4ERR_BAD_STATEID should be returned.

This error condition will occur when there has been a logic error on the part of the client or server. This should not happen.

One mechanism that may be used to satisfy these requirements is for the server to,

o divide the "other" field of each stateid into two fields:

- A server verifier which uniquely designates a particular server instantiation.

- An index into a table of locking-state structures. 
o utilize the "seqid" field of each stateid, such that seqid is monotonically incremented for each stateid that is associated with the same index into the locking-state table.

By matching the incoming stateid and its field values with the state held at the server, the server is able to easily determine if a stateid is valid for its current instantiation and state. If the stateid is not valid, the appropriate error can be supplied to the client.

8.1.4. Use of the stateid and Locking

All READ, WRITE and SETATTR operations contain a stateid. For the purposes of this section, SETATTR operations which change the size attribute of a file are treated as if they are writing the area between the old and new size (i.e., the range truncated or added to the file by means of the SETATTR), even where SETATTR is not explicitly mentioned in the text.

If the lock_owner performs a READ or WRITE in a situation in which it has established a lock or share reservation on the server (any OPEN constitutes a share reservation) the stateid (previously returned by the server) must be used to indicate what locks, including both record locks and share reservations, are held by the lockowner. If no state is established by the client, either record lock or share reservation, a stateid of all bits 0 is used. Regardless whether a stateid of all bits 0 , or a stateid returned by the server is used, if there is a conflicting share reservation or mandatory record lock held on the file, the server MUST refuse to service the READ or WRITE operation.

Share reservations are established by OPEN operations and by their nature are mandatory in that when the OPEN denies READ or WRITE operations, that denial results in such operations being rejected with error NFS4ERR_LOCKED. Record locks may be implemented by the server as either mandatory or advisory, or the choice of mandatory or advisory behavior may be determined by the server on the basis of the file being accessed (for example, some UNIX-based servers support a "mandatory lock bit" on the mode attribute such that if set, record locks are required on the file before I/O is possible). When record locks are advisory, they only prevent the granting of conflicting lock requests and have no effect on READs or WRITEs. Mandatory record locks, however, prevent conflicting I/O operations. When they are attempted, they are rejected with NFS4ERR_LOCKED. When the client gets NFS4ERR_LOCKED on a file it knows it has the proper share reservation for, it will need to issue a LOCK request on the region 
of the file that includes the region the I/O was to be performed on, with an appropriate locktype (i.e., READ*_LT for a READ operation, WRITE*_LT for a WRITE operation).

With NFS version 3, there was no notion of a stateid so there was no way to tell if the application process of the client sending the READ or WRITE operation had also acquired the appropriate record lock on the file. Thus there was no way to implement mandatory locking. With the stateid construct, this barrier has been removed.

Note that for UNIX environments that support mandatory file locking, the distinction between advisory and mandatory locking is subtle. In fact, advisory and mandatory record locks are exactly the same in so far as the APIs and requirements on implementation. If the mandatory lock attribute is set on the file, the server checks to see if the lockowner has an appropriate shared (read) or exclusive (write) record lock on the region it wishes to read or write to. If there is no appropriate lock, the server checks if there is a conflicting lock (which can be done by attempting to acquire the conflicting lock on the behalf of the lockowner, and if successful, release the lock after the READ or WRITE is done), and if there is, the server returns NFS 4ERR_LOCKED.

For Windows environments, there are no advisory record locks, so the server always checks for record locks during I/O requests.

Thus, the NFS version 4 LOCK operation does not need to distinguish between advisory and mandatory record locks. It is the NFS version 4 server's processing of the READ and WRITE operations that introduces the distinction.

Every stateid other than the special stateid values noted in this section, whether returned by an OPEN-type operation (i.e., OPEN, OPEN_DOWNGRADE), or by a LOCK-type operation (i.e., LOCK or LOCKU), defines an access mode for the file (i.e., READ, WRITE, or READWRITE) as established by the original OPEN which began the stateid sequence, and as modified by subsequent OPENs and OPEN_DOWNGRADEs within that stateid sequence. When a READ, WRITE, or SETATTR which specifies the size attribute, is done, the operation is subject to checking against the access mode to verify that the operation is appropriate given the OPEN with which the operation is associated.

In the case of WRITE-type operations (i.e., WRITEs and SETATTRs which set size), the server must verify that the access mode allows writing and return an NFS4ERR_OPENMODE error if it does not. In the case, of READ, the server may perform the corresponding check on the access mode, or it may choose to allow READ on opens for WRITE only, to accommodate clients whose write implementation may unavoidably do 
reads (e.g., due to buffer cache constraints). However, even if READs are allowed in these circumstances, the server MUST still check for locks that conflict with the READ (e.g., another open specify denial of READs). Note that a server which does enforce the access mode check on READs need not explicitly check for conflicting share reservations since the existence of OPEN for read access guarantees that no conflicting share reservation can exist.

A stateid of all bits 1 (one) MAY allow READ operations to bypass locking checks at the server. However, WRITE operations with a stateid with bits all 1 (one) MUST NOT bypass locking checks and are treated exactly the same as if a stateid of all bits 0 were used.

A lock may not be granted while a READ or WRITE operation using one of the special stateids is being performed and the range of the lock request conflicts with the range of the READ or WRITE operation. For the purposes of this paragraph, a conflict occurs when a shared lock is requested and a WRITE operation is being performed, or an exclusive lock is requested and either a READ or a WRITE operation is being performed. A SETATTR that sets size is treated similarly to a WRITE as discussed above.

\subsubsection{Sequencing of Lock Requests}

Locking is different than most NFS operations as it requires "atmost-one" semantics that are not provided by ONCRPC. ONCRPC over a reliable transport is not sufficient because a sequence of locking requests may span multiple TCP connections. In the face of retransmission or reordering, lock or unlock requests must have a well defined and consistent behavior. To accomplish this, each lock request contains a sequence number that is a consecutively increasing integer. Different lock_owners have different sequences. The server maintains the last sequence number (L) received and the response that was returned. The first request issued for any given lock_owner is issued with a sequence number of zero.

Note that for requests that contain a sequence number, for each lock_owner, there should be no more than one outstanding request.

If a request ( $r$ ) with a previous sequence number $(r<L)$ is received, it is rejected with the return of error NFS4ERR_BAD_SEQID. Given a properly-functioning client, the response to (r) must have been received before the last request (L) was sent. If a duplicate of last request $(r==L)$ is received, the stored response is returned. If a request beyond the next sequence $(r==L+2)$ is received, it is rejected with the return of error NFS4ERR_BAD_SEQID. Sequence history is reinitialized whenever the SETCLIENTID/SETCLIENTID_CONFIRM sequence changes the client verifier. 
Since the sequence number is represented with an unsigned 32-bit integer, the arithmetic involved with the sequence number is mod 2^32. For an example of modulo arithmetic involving sequence numbers see $[R F C 793]$.

It is critical the server maintain the last response sent to the client to provide a more reliable cache of duplicate non-idempotent requests than that of the traditional cache described in [Juszczak]. The traditional duplicate request cache uses a least recently used algorithm for removing unneeded requests. However, the last lock request and response on a given lock_owner must be cached as long as the lock state exists on the server.

The client MUST monotonically increment the sequence number for the CLOSE, LOCK, LOCKU, OPEN, OPEN_CONFIRM, and OPEN_DOWNGRADE operations. This is true even in the event that the previous operation that used the sequence number received an error. The only exception to this rule is if the previous operation received one of the following errors: NFS4ERR_STALE_CLIENTID, NFS4ERR_STALE_STATEID, NFS4ERR_BAD_STATEID, NFS4ERR_BAD_SEQID， NFS4ERR_BADXDR, NFS 4ERR_RESOURCE, NFS4ERR_NOFILEHANDLE.

8.1.6. Recovery from Replayed Requests

As described above, the sequence number is per lock_owner. As long as the server maintains the last sequence number received and follows the methods described above, there are no risks of a Byzantine router re-sending old requests. The server need only maintain the (lock_owner, sequence number) state as long as there are open files or closed files with locks outstanding.

LOCK, LOCKU, OPEN, OPEN_DOWNGRADE, and CLOSE each contain a sequence number and therefore the risk of the replay of these operations resulting in undesired effects is non-existent while the server maintains the lock_owner state.

\subsubsection{Releasing lock_owner State}

When a particular lock_owner no longer holds open or file locking state at the server, the server may choose to release the sequence number state associated with the lock_owner. The server may make this choice based on lease expiration, for the reclamation of server memory, or other implementation specific details. In any event, the server is able to do this safely only when the lock_owner no longer is being utilized by the client. The server may choose to hold the lock_owner state in the event that retransmitted requests are received. However, the period to hold this state is implementation specific. 
In the case that a LOCK, LOCKU, OPEN_DOWNGRADE, or CLOSE is retransmitted after the server has previously released the lock_owner state, the server will find that the lock_owner has no files open and an error will be returned to the client. If the lock_owner does have a file open, the stateid will not match and again an error is returned to the client.

8.1.8. Use of Open Confirmation

In the case that an OPEN is retransmitted and the lock_owner is being used for the first time or the lock_owner state has been previously released by the server, the use of the OPEN_CONFIRM operation will prevent incorrect behavior. When the server observes the use of the lock_owner for the first time, it will direct the client to perform the OPEN_CONFIRM for the corresponding OPEN. This sequence establishes the use of an lock_owner and associated sequence number. Since the OPEN_CONFIRM sequence connects a new open_owner on the server with an existing open_owner on a client, the sequence number may have any value. The OPEN_CONFIRM step assures the server that the value received is the correct one. See the section "OPEN_CONFIRM - Confirm Open" for further details.

There are a number of situations in which the requirement to confirm an OPEN would pose difficulties for the client and server, in that they would be prevented from acting in a timely fashion on information received, because that information would be provisional, subject to deletion upon non-confirmation. Fortunately, these are situations in which the server can avoid the need for confirmation when responding to open requests. The two constraints are:

o The server must not bestow a delegation for any open which would require confirmation.

- The server MUST NOT require confirmation on a reclaim-type open (i.e., one specifying claim type CLAIM_PREVIOUS or CLAIM_DELEGATE_PREV).

These constraints are related in that reclaim-type opens are the only ones in which the server may be required to send a delegation. For CLAIM_NULL, sending the delegation is optional while for CLAIM_DELEGATE_CUR, no delegation is sent.

Delegations being sent with an open requiring confirmation are troublesome because recovering from non-confirmation adds undue complexity to the protocol while requiring confirmation on reclaimtype opens poses difficulties in that the inability to resolve 
the status of the reclaim until lease expiration may make it difficult to have timely determination of the set of locks being reclaimed (since the grace period may expire).

Requiring open confirmation on reclaim-type opens is avoidable because of the nature of the environments in which such opens are done. For CLAIM_PREVIOUS opens, this is immediately after server reboot, so there should be no time for lockowners to be created, found to be unused, and recycled. For CLAIM_DELEGATE_PREV opens, we are dealing with a client reboot situation. A server which supports delegation can be sure that no lockowners for that client have been recycled since client initialization and thus can ensure that confirmation will not be required.

\subsection{Lock Ranges}

The protocol allows a lock owner to request a lock with a byte range and then either upgrade or unlock a sub-range of the initial lock. It is expected that this will be an uncommon type of request. In any case, servers or server filesystems may not be able to support subrange lock semantics. In the event that a server receives a locking request that represents a sub-range of current locking state for the lock owner, the server is allowed to return the error NFS4ERR_LOCK_RANGE to signify that it does not support sub-range lock operations. Therefore, the client should be prepared to receive this error and, if appropriate, report the error to the requesting application.

The client is discouraged from combining multiple independent locking ranges that happen to be adjacent into a single request since the server may not support sub-range requests and for reasons related to the recovery of file locking state in the event of server failure. As discussed in the section "Server Failure and Recovery" below, the server may employ certain optimizations during recovery that work effectively only when the client's behavior during lock recovery is similar to the client's locking behavior prior to server failure.

\subsection{Upgrading and Downgrading Locks}

If a client has a write lock on a record, it can request an atomic downgrade of the lock to a read lock via the Lock request, by setting the type to READ_LT. If the server supports atomic downgrade, the request will succeed. If not, it will return NFS4ERR_LOCK_NOTSUPP . The client should be prepared to receive this error, and if appropriate, report the error to the requesting application. 
If a client has a read lock on a record, it can request an atomic upgrade of the lock to a write lock via the LoCk request by setting the type to WRITE_LT or WRITEW_LT. If the server does not support atomic upgrade, it will return NFS4ERR_LOCK_NOTSUPP. If the upgrade can be achieved without an existing conflict, the request will succeed. Otherwise, the server will return either NFS4ERR_DENIED or NFS4ERR_DEADLOCK. The error NFS4ERR_DEADLOCK is returned if the client issued the LOCK request with the type set to WRITEW_LT and the server has detected a deadlock. The client should be prepared to receive such errors and if appropriate, report the error to the requesting application.

\subsection{Blocking Locks}

Some clients require the support of blocking locks. The NFS version 4 protocol must not rely on a callback mechanism and therefore is unable to notify a client when a previously denied lock has been granted. Clients have no choice but to continually poll for the lock. This presents a fairness problem. Two new lock types are added, READW and WRITEW, and are used to indicate to the server that the client is requesting a blocking lock. The server should maintain an ordered list of pending blocking locks. When the conflicting lock is released, the server may wait the lease period for the first waiting client to re-request the lock. After the lease period expires the next waiting client request is allowed the lock. Clients are required to poll at an interval sufficiently small that it is likely to acquire the lock in a timely manner. The server is not required to maintain a list of pending blocked locks as it is used to increase fairness and not correct operation. Because of the unordered nature of crash recovery, storing of lock state to stable storage would be required to guarantee ordered granting of blocking locks.

Servers may also note the lock types and delay returning denial of the request to allow extra time for a conflicting lock to be released, allowing a successful return. In this way, clients can avoid the burden of needlessly frequent polling for blocking locks. The server should take care in the length of delay in the event the client retransmits the request.

\subsection{Lease Renewal}

The purpose of a lease is to allow a server to remove stale locks that are held by a client that has crashed or is otherwise unreachable. It is not a mechanism for cache consistency and lease renewals may not be denied if the lease interval has not expired. 
The following events cause implicit renewal of all of the leases for a given client (i.e., all those sharing a given clientid). Each of these is a positive indication that the client is still active and that the associated state held at the server, for the client, is still valid.

- An OPEN with a valid clientid.

- Any operation made with a valid stateid (CLOSE, DELEGPURGE, DELEGRETURN, LOCK, LOCKU, OPEN, OPEN_CONFIRM, OPEN_DOWNGRADE, READ, RENEW, SETATTR, WRITE). This does not include the special stateids of all bits 0 or all bits 1 .

Note that if the client had restarted or rebooted, the client would not be making these requests without issuing the SETCLIENTID/SETCLIENTID_CONFIRM sequence. The use of the SETCLIENTID/SETCLIENTID_CONFIRM sequence (one that changes the client verifier) notifies the server to drop the locking state associated with the client. SETCLIENTID/SETCLIENTID_CONFIRM never renews a lease.

If the server has rebooted, the stateids (NFS4ERR_STALE_STATEID error) or the clientid (NFS4ERR_STALE_CLIENTID error) will not be valid hence preventing spurious renewals.

This approach allows for low overhead lease renewal which scales well. In the typical case no extra RPC calls are required for lease renewal and in the worst case one RPC is required every lease period (i.e., a RENEW operation). The number of locks held by the client is not a factor since all state for the client is involved with the lease renewal action.

Since all operations that create a new lease also renew existing leases, the server must maintain a common lease expiration time for all valid leases for a given client. This lease time can then be easily updated upon implicit lease renewal actions.

\subsection{Crash Recovery}

The important requirement in crash recovery is that both the client and the server know when the other has failed. Additionally, it is required that a client sees a consistent view of data across server restarts or reboots. All READ and WRITE operations that may have been queued within the client or network buffers must wait until the client has successfully recovered the locks protecting the READ and WRITE operations. 


\subsubsection{Client Failure and Recovery}

In the event that a client fails, the server may recover the client's locks when the associated leases have expired. Conflicting locks from another client may only be granted after this lease expiration. If the client is able to restart or reinitialize within the lease period the client may be forced to wait the remainder of the lease period before obtaining new locks.

To minimize client delay upon restart, lock requests are associated with an instance of the client by a client supplied verifier. This verifier is part of the initial SETCLIENTID call made by the client. The server returns a clientid as a result of the SETCLIENTID

operation. The client then confirms the use of the clientid with SETCLIENTID_CONFIRM. The clientid in combination with an opaque owner field is then used by the client to identify the lock owner for OPEN. This chain of associations is then used to identify all locks for a particular client.

Since the verifier will be changed by the client upon each initialization, the server can compare a new verifier to the verifier associated with currently held locks and determine that they do not match. This signifies the client's new instantiation and subsequent loss of locking state. As a result, the server is free to release all locks held which are associated with the old clientid which was derived from the old verifier.

Note that the verifier must have the same uniqueness properties of the verifier for the COMMIT operation.

\subsubsection{Server Failure and Recovery}

If the server loses locking state (usually as a result of a restart or reboot), it must allow clients time to discover this fact and reestablish the lost locking state. The client must be able to reestablish the locking state without having the server deny valid requests because the server has granted conflicting access to another client. Likewise, if there is the possibility that clients have not yet re-established their locking state for a file, the server must disallow READ and WRITE operations for that file. The duration of this recovery period is equal to the duration of the lease period.

A client can determine that server failure (and thus loss of locking state) has occurred, when it receives one of two errors. The NFS4ERR_STALE_STATEID error indicates a stateid invalidated by a reboot or restart. The NFS4ERR_STALE_CLIENTID error indicates a 
clientid invalidated by reboot or restart. When either of these are received, the client must establish a new clientid (See the section "Client ID") and re-establish the locking state as discussed below.

The period of special handling of locking and READs and WRITEs, equal in duration to the lease period, is referred to as the "grace period". During the grace period, clients recover locks and the associated state by reclaim-type locking requests (i.e., LOCK requests with reclaim set to true and OPEN operations with a claim type of CLAIM_PREVIOUS). During the grace period, the server must reject READ and WRITE operations and non-reclaim locking requests (i.e., other LOCK and OPEN operations) with an error of NFS 4ERR_GRACE.

If the server can reliably determine that granting a non-reclaim request will not conflict with reclamation of locks by other clients, the NFS4ERR_GRACE error does not have to be returned and the nonreclaim client request can be serviced. For the server to be able to service READ and WRITE operations during the grace period, it must again be able to guarantee that no possible conflict could arise between an impending reclaim locking request and the READ or WRITE operation. If the server is unable to offer that guarantee, the NFS4ERR_GRACE error must be returned to the client.

For a server to provide simple, valid handling during the grace period, the easiest method is to simply reject all non-reclaim locking requests and READ and WRITE operations by returning the NFS4ERR_GRACE error. However, a server may keep information about granted locks in stable storage. With this information, the server could determine if a regular lock or READ or WRITE operation can be safely processed.

For example, if a count of locks on a given file is available in stable storage, the server can track reclaimed locks for the file and when all reclaims have been processed, non-reclaim locking requests may be processed. This way the server can ensure that non-reclaim locking requests will not conflict with potential reclaim requests. With respect to I/O requests, if the server is able to determine that there are no outstanding reclaim requests for a file by information from stable storage or another similar mechanism, the processing of I/O requests could proceed normally for the file.

To reiterate, for a server that allows non-reclaim lock and I/O requests to be processed during the grace period, it MUST determine that no lock subsequently reclaimed will be rejected and that no lock subsequently reclaimed would have prevented any I/O operation processed during the grace period. 
Clients should be prepared for the return of NFS4ERR_GRACE errors for non-reclaim lock and I/O requests. In this case the client should employ a retry mechanism for the request. A delay (on the order of several seconds) between retries should be used to avoid overwhelming the server. Further discussion of the general issue is included in [Floyd]. The client must account for the server that is able to perform I/O and non-reclaim locking requests within the grace period as well as those that can not do so.

A reclaim-type locking request outside the server's grace period can only succeed if the server can guarantee that no conflicting lock or I/O request has been granted since reboot or restart.

A server may, upon restart, establish a new value for the lease period. Therefore, clients should, once a new clientid is established, refetch the lease_time attribute and use it as the basis for lease renewal for the lease associated with that server. However, the server must establish, for this restart event, a grace period at least as long as the lease period for the previous server instantiation. This allows the client state obtained during the previous server instance to be reliably re-established.

\subsubsection{Network Partitions and Recovery}

If the duration of a network partition is greater than the lease period provided by the server, the server will have not received a lease renewal from the client. If this occurs, the server may free all locks held for the client. As a result, all stateids held by the client will become invalid or stale. Once the client is able to reach the server after such a network partition, all I/O submitted by the client with the now invalid stateids will fail with the server returning the error NFS4ERR_EXPIRED. Once this error is received, the client will suitably notify the application that held the lock.

As a courtesy to the client or as an optimization, the server may continue to hold locks on behalf of a client for which recent communication has extended beyond the lease period. If the server receives a lock or I/O request that conflicts with one of these courtesy locks, the server must free the courtesy lock and grant the new request.

When a network partition is combined with a server reboot, there are edge conditions that place requirements on the server in order to avoid silent data corruption following the server reboot. Two of these edge conditions are known, and are discussed below. 
The first edge condition has the following scenario:

1. Client $A$ acquires a lock.

2. Client $A$ and server experience mutual network partition, such that client $A$ is unable to renew its lease.

3. Client $A^{\prime}$ s lease expires, so server releases lock.

4. Client B acquires a lock that would have conflicted with that of Client $\mathrm{A}$.

5. Client $B$ releases the lock

6. Server reboots

7. Network partition between client $A$ and server heals.

8. Client A issues a RENEW operation, and gets back a NFS4ERR_STALE_CLIENTID.

9. Client A reclaims its lock within the server's grace period. Thus, at the final step, the server has erroneously granted client $A^{\prime}$ s lock reclaim. If client $B$ modified the object the lock was protecting, client A will experience object corruption.

The second known edge condition follows:

1. Client $A$ acquires a lock.

2. Server reboots.

3. Client $A$ and server experience mutual network partition, such that client $A$ is unable to reclaim its lock within the grace period.

4. Server's reclaim grace period ends. Client A has no locks recorded on server.

5. Client B acquires a lock that would have conflicted with that of Client A.

6. Client $B$ releases the lock.

7. Server reboots a second time.

8. Network partition between client $A$ and server heals. 
9. Client A issues a RENEW operation, and gets back a NFS4ERR_STALE_CLIENTID.

10. Client A reclaims its lock within the server's grace period.

As with the first edge condition, the final step of the scenario of the second edge condition has the server erroneously granting client $A^{\prime}$ s lock reclaim.

Solving the first and second edge conditions requires that the server either assume after it reboots that edge condition occurs, and thus return NFS4ERR_NO_GRACE for all reclaim attempts, or that the server record some information stable storage. The amount of information the server records in stable storage is in inverse proportion to how harsh the server wants to be whenever the edge conditions occur. The server that is completely tolerant of all edge conditions will record in stable storage every lock that is acquired, removing the lock record from stable storage only when the lock is unlocked by the client and the lock's lockowner advances the sequence number such that the lock release is not the last stateful event for the lockowner's sequence. For the two aforementioned edge conditions, the harshest a server can be, and still support a grace period for reclaims, requires that the server record in stable storage information some minimal information. For example, a server implementation could, for each client, save in stable storage a record containing:

o the client's id string

- a boolean that indicates if the client's lease expired or if there was administrative intervention (see the section, Server Revocation of Locks) to revoke a record lock, share reservation, or delegation

- a timestamp that is updated the first time after a server boot or reboot the client acquires record locking, share reservation, or delegation state on the server. The timestamp need not be updated on subsequent lock requests until the server reboots.

The server implementation would also record in the stable storage the timestamps from the two most recent server reboots.

Assuming the above record keeping, for the first edge condition, after the server reboots, the record that client $A^{\prime}$ s lease expired means that another client could have acquired a conflicting record lock, share reservation, or delegation. Hence the server must reject a reclaim from client $A$ with the error NFS4ERR_NO_GRACE. 
For the second edge condition, after the server reboots for a second time, the record that the client had an unexpired record lock, share reservation, or delegation established before the server's previous incarnation means that the server must reject a reclaim from client $A$ with the error NFS4ERR_NO_GRACE.

Regardless of the level and approach to record keeping, the server MUST implement one of the following strategies (which apply to reclaims of share reservations, record locks, and delegations):

1. Reject all reclaims with NFS4ERR_NO_GRACE. This is superharsh, but necessary if the server does not want to record lock state in stable storage.

2. Record sufficient state in stable storage such that all known edge conditions involving server reboot, including the two noted in this section, are detected. False positives are acceptable. Note that at this time, it is not known if there are other edge conditions.

In the event, after a server reboot, the server determines that there is unrecoverable damage or corruption to the the stable storage, then for all clients and/or locks affected, the server MUST return NFS4ERR_NO_GRACE.

A mandate for the client's handling of the NFS4ERR_NO_GRACE error is outside the scope of this specification, since the strategies for such handling are very dependent on the client's operating environment. However, one potential approach is described below.

When the client receives NFS4ERR_NO_GRACE, it could examine the change attribute of the objects the client is trying to reclaim state for, and use that to determine whether to re-establish the state via normal OPEN or LOCK requests. This is acceptable provided the client's operating environment allows it. In otherwords, the client implementor is advised to document for his users the behavior. The client could also inform the application that its record lock or share reservations (whether they were delegated or not) have been lost, such as via a UNIX signal, a GUI pop-up window, etc. See the section, "Data Caching and Revocation" for a discussion of what the client should do for dealing with unreclaimed delegations on client state.

For further discussion of revocation of locks see the section "Server Revocation of Locks". 
8.7. Recovery from a Lock Request Timeout or Abort

In the event a lock request times out, a client may decide to not retry the request. The client may also abort the request when the process for which it was issued is terminated (e.g., in UNIX due to a signal). It is possible though that the server received the request and acted upon it. This would change the state on the server without the client being aware of the change. It is paramount that the client re-synchronize state with server before it attempts any other operation that takes a seqid and/or a stateid with the same lock_owner. This is straightforward to do without a special resynchronize operation.

Since the server maintains the last lock request and response received on the lock_owner, for each lock_owner, the client should cache the last lock request it sent such that the lock request did not receive a response. From this, the next time the client does a lock operation for the lock_owner, it can send the cached request, if there is one, and if the request was one that established state (e.g., a LOCK or OPEN operation), the server will return the cached result or if never saw the request, perform it. The client can follow up with a request to remove the state (e.g., a LOCKU or CLOSE operation). With this approach, the sequencing and stateid information on the client and server for the given lock_owner will re-synchronize and in turn the lock state will re-synchronize.

\subsection{Server Revocation of Locks}

At any point, the server can revoke locks held by a client and the client must be prepared for this event. When the client detects that its locks have been or may have been revoked, the client is responsible for validating the state information between itself and the server. Validating locking state for the client means that it must verify or reclaim state for each lock currently held.

The first instance of lock revocation is upon server reboot or reinitialization. In this instance the client will receive an error (NFS4ERR_STALE_STATEID or NFS4ERR_STALE_CLIENTID) and the client will proceed with normal crash recovery as described in the previous section.

The second lock revocation event is the inability to renew the lease before expiration. While this is considered a rare or unusual event, the client must be prepared to recover. Both the server and client will be able to detect the failure to renew the lease and are capable of recovering without data corruption. For the server, it tracks the last renewal event serviced for the client and knows when the lease will expire. Similarly, the client must track operations which will 
renew the lease period. Using the time that each such request was sent and the time that the corresponding reply was received, the client should bound the time that the corresponding renewal could have occurred on the server and thus determine if it is possible that a lease period expiration could have occurred.

The third lock revocation event can occur as a result of administrative intervention within the lease period. While this is considered a rare event, it is possible that the server's administrator has decided to release or revoke a particular lock held by the client. As a result of revocation, the client will receive an error of NFS4ERR_ADMIN_REVOKED. In this instance the client may assume that only the lock_owner's locks have been lost. The client notifies the lock holder appropriately. The client may not assume the lease period has been renewed as a result of failed operation.

When the client determines the lease period may have expired, the client must mark all locks held for the associated lease as "unvalidated". This means the client has been unable to re-establish or confirm the appropriate lock state with the server. As described in the previous section on crash recovery, there are scenarios in which the server may grant conflicting locks after the lease period has expired for a client. When it is possible that the lease period has expired, the client must validate each lock currently held to ensure that a conflicting lock has not been granted. The client may accomplish this task by issuing an I/O request, either a pending I/O or a zero-length read, specifying the stateid associated with the lock in question. If the response to the request is success, the client has validated all of the locks governed by that stateid and re-established the appropriate state between itself and the server.

If the I/O request is not successful, then one or more of the locks associated with the stateid was revoked by the server and the client must notify the owner.

\subsection{Share Reservations}

A share reservation is a mechanism to control access to a file. It is a separate and independent mechanism from record locking. When a client opens a file, it issues an OPEN operation to the server specifying the type of access required (READ, WRITE, or BOTH) and the type of access to deny others (deny NONE, READ, WRITE, or BOTH). If the OPEN fails the client will fail the application's open request.

Pseudo-code definition of the semantics:

if (request. access $==0$ ) return (NFS4ERR_INVAL) 


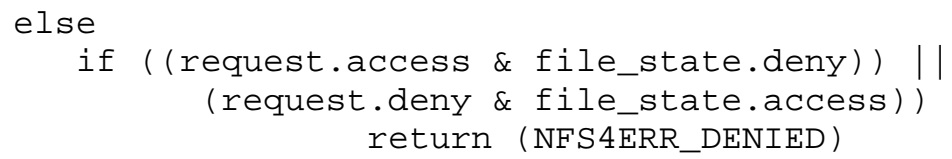

This checking of share reservations on OPEN is done with no exception for an existing OPEN for the same open_owner.

The constants used for the OPEN and OPEN_DOWNGRADE operations for the access and deny fields are as follows:

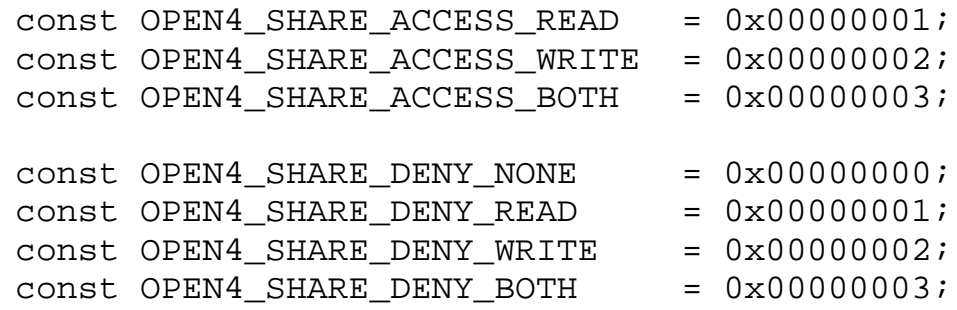

\subsection{OPEN/CLOSE Operations}

To provide correct share semantics, a client MUST use the OPEN operation to obtain the initial filehandle and indicate the desired access and what if any access to deny. Even if the client intends to use a stateid of all $0^{\prime} \mathrm{s}$ or all 1's, it must still obtain the filehandle for the regular file with the OPEN operation so the appropriate share semantics can be applied. For clients that do not have a deny mode built into their open programming interfaces, deny equal to NONE should be used.

The OPEN operation with the CREATE flag, also subsumes the CREATE operation for regular files as used in previous versions of the NFS protocol. This allows a create with a share to be done atomically.

The CLOSE operation removes all share reservations held by the lock_owner on that file. If record locks are held, the client SHOULD release all locks before issuing a CLOSE. The server MAY free all outstanding locks on CLOSE but some servers may not support the CLOSE of a file that still has record locks held. The server MUST return failure, NFS4ERR_LOCKS_HELD, if any locks would exist after the CLOSE.

The LoOKUP operation will return a filehandle without establishing any lock state on the server. Without a valid stateid, the server will assume the client has the least access. For example, a file 
opened with deny READ/WRITE cannot be accessed using a filehandle obtained through LOOKUP because it would not have a valid stateid (i.e., using a stateid of all bits 0 or all bits 1).

8.10.1. Close and Retention of State Information

Since a CLOSE operation requests deallocation of a stateid, dealing with retransmission of the CLOSE, may pose special difficulties, since the state information, which normally would be used to determine the state of the open file being designated, might be deallocated, resulting in an NFS4ERR_BAD_STATEID error.

Servers may deal with this problem in a number of ways. To provide the greatest degree assurance that the protocol is being used properly, a server should, rather than deallocate the stateid, mark it as close-pending, and retain the stateid with this status, until later deallocation. In this way, a retransmitted CLOSE can be recognized since the stateid points to state information with this distinctive status, so that it can be handled without error.

When adopting this strategy, a server should retain the state information until the earliest of:

- Another validly sequenced request for the same lockowner, that is not a retransmission.

- The time that a lockowner is freed by the server due to period with no activity.

- All locks for the client are freed as a result of a SETCLIENTID.

Servers may avoid this complexity, at the cost of less complete protocol error checking, by simply responding NFS4_OK in the event of a CLOSE for a deallocated stateid, on the assumption that this case must be caused by a retransmitted close. When adopting this approach, it is desirable to at least log an error when returning a no-error indication in this situation. If the server maintains a reply-cache mechanism, it can verify the CLOSE is indeed a retransmission and avoid error logging in most cases.

8.11. Open Upgrade and Downgrade

When an OPEN is done for a file and the lockowner for which the open is being done already has the file open, the result is to upgrade the open file status maintained on the server to include the access and deny bits specified by the new OPEN as well as those for the existing OPEN. The result is that there is one open file, as far as the protocol is concerned, and it includes the union of the access and 
deny bits for all of the OPEN requests completed. Only a single CLOSE will be done to reset the effects of both OPENs. Note that the client, when issuing the OPEN, may not know that the same file is in fact being opened. The above only applies if both opeNs result in the OPENed object being designated by the same filehandle.

When the server chooses to export multiple filehandles corresponding to the same file object and returns different filehandles on two different OPENs of the same file object, the server MUST NOT "OR" together the access and deny bits and coalesce the two open files. Instead the server must maintain separate OPENs with separate stateids and will require separate CLOSEs to free them.

When multiple open files on the client are merged into a single open file object on the server, the close of one of the open files (on the client) may necessitate change of the access and deny status of the open file on the server. This is because the union of the access and deny bits for the remaining opens may be smaller (i.e., a proper subset) than previously. The OPEN_DOWNGRADE operation is used to make the necessary change and the client should use it to update the server so that share reservation requests by other clients are handled properly.

\subsection{Short and Long Leases}

When determining the time period for the server lease, the usual lease tradeoffs apply. Short leases are good for fast server recovery at a cost of increased RENEW or READ (with zero length) requests. Longer leases are certainly kinder and gentler to servers trying to handle very large numbers of clients. The number of RENEW requests drop in proportion to the lease time. The disadvantages of long leases are slower recovery after server failure (the server must wait for the leases to expire and the grace period to elapse before granting new lock requests) and increased file contention (if client fails to transmit an unlock request then server must wait for lease expiration before granting new locks).

Long leases are usable if the server is able to store lease state in non-volatile memory. Upon recovery, the server can reconstruct the lease state from its non-volatile memory and continue operation with its clients and therefore long leases would not be an issue.

\subsection{Clocks, Propagation Delay, and Calculating Lease Expiration}

To avoid the need for synchronized clocks, lease times are granted by the server as a time delta. However, there is a requirement that the client and server clocks do not drift excessively over the duration of the lock. There is also the issue of propagation delay across the 
network which could easily be several hundred milliseconds as well as the possibility that requests will be lost and need to be retransmitted.

To take propagation delay into account, the client should subtract it from lease times (e.g., if the client estimates the one-way propagation delay as $200 \mathrm{msec}$, then it can assume that the lease is already $200 \mathrm{msec}$ old when it gets it). In addition, it will take another $200 \mathrm{msec}$ to get a response back to the server. So the client must send a lock renewal or write data back to the server $400 \mathrm{msec}$ before the lease would expire.

The server's lease period configuration should take into account the network distance of the clients that will be accessing the server's resources. It is expected that the lease period will take into account the network propagation delays and other network delay factors for the client population. Since the protocol does not allow for an automatic method to determine an appropriate lease period, the server's administrator may have to tune the lease period.

\subsection{Migration, Replication and state}

When responsibility for handling a given file system is transferred to a new server (migration) or the client chooses to use an alternate server (e.g., in response to server unresponsiveness) in the context of file system replication, the appropriate handling of state shared between the client and server (i.e., locks, leases, stateids, and clientids) is as described below. The handling differs between migration and replication. For related discussion of file server state and recover of such see the sections under "File Locking and Share Reservations".

If server replica or a server immigrating a filesystem agrees to, or is expected to, accept opaque values from the client that originated from another server, then it is a wise implementation practice for the servers to encode the "opaque" values in network byte order. This way, servers acting as replicas or immigrating filesystems will be able to parse values like stateids, directory cookies, filehandles, etc. even if their native byte order is different from other servers cooperating in the replication and migration of the filesystem.

\subsubsection{Migration and State}

In the case of migration, the servers involved in the migration of a filesystem SHOULD transfer all server state from the original to the new server. This must be done in a way that is transparent to the client. This state transfer will ease the client's transition when a 
filesystem migration occurs. If the servers are successful in transferring all state, the client will continue to use stateids assigned by the original server. Therefore the new server must recognize these stateids as valid. This holds true for the clientid as well. Since responsibility for an entire filesystem is transferred with a migration event, there is no possibility that conflicts will arise on the new server as a result of the transfer of locks.

As part of the transfer of information between servers, leases would be transferred as well. The leases being transferred to the new server will typically have a different expiration time from those for the same client, previously on the old server. To maintain the property that all leases on a given server for a given client expire at the same time, the server should advance the expiration time to the later of the leases being transferred or the leases already present. This allows the client to maintain lease renewal of both classes without special effort.

The servers may choose not to transfer the state information upon migration. However, this choice is discouraged. In this case, when the client presents state information from the original server, the client must be prepared to receive either NFS4ERR_STALE_CLIENTID or NFS4ERR_STALE_STATEID from the new server. The client should then recover its state information as it normally would in response to a server failure. The new server must take care to allow for the recovery of state information as it would in the event of server restart.

\subsubsection{Replication and state}

Since client switch-over in the case of replication is not under server control, the handling of state is different. In this case, leases, stateids and clientids do not have validity across a transition from one server to another. The client must re-establish its locks on the new server. This can be compared to the reestablishment of locks by means of reclaim-type requests after a server reboot. The difference is that the server has no provision to distinguish requests reclaiming locks from those obtaining new locks or to defer the latter. Thus, a client re-establishing a lock on the new server (by means of a LOCK or OPEN request), may have the requests denied due to a conflicting lock. Since replication is intended for read-only use of filesystems, such denial of locks should not pose large difficulties in practice. When an attempt to re-establish a lock on a new server is denied, the client should treat the situation as if his original lock had been revoked. 


\subsubsection{Notification of Migrated Lease}

In the case of lease renewal, the client may not be submitting requests for a filesystem that has been migrated to another server. This can occur because of the implicit lease renewal mechanism. The client renews leases for all filesystems when submitting a request to any one filesystem at the server.

In order for the client to schedule renewal of leases that may have been relocated to the new server, the client must find out about lease relocation before those leases expire. To accomplish this, all operations which implicitly renew leases for a client (i.e., OPEN, CLOSE, READ, WRITE, RENEW, LOCK, LOCKT, LOCKU), will return the error NFS4ERR_LEASE_MOVED if responsibility for any of the leases to be renewed has been transferred to a new server. This condition will continue until the client receives an NFS4ERR_MOVED error and the server receives the subsequent GETATTR(fs_locations) for an access to each filesystem for which a lease has been moved to a new server.

When a client receives an NFS4ERR_LEASE_MOVED error, it should perform an operation on each filesystem associated with the server in question. When the client receives an NFS4ERR_MOVED error, the client can follow the normal process to obtain the new server information (through the fs_locations attribute) and perform renewal of those leases on the new server. If the server has not had state transferred to it transparently, the client will receive either NFS4ERR_STALE_CLIENTID or NFS4ERR_STALE_STATEID from the new server, as described above, and the client can then recover state information as it does in the event of server failure.

\subsubsection{Migration and the Lease_time Attribute}

In order that the client may appropriately manage its leases in the case of migration, the destination server must establish proper values for the lease_time attribute.

When state is transferred transparently, that state should include the correct value of the lease_time attribute. The lease_time attribute on the destination server must never be less than that on the source since this would result in premature expiration of leases granted by the source server. Upon migration in which state is transferred transparently, the client is under no obligation to refetch the lease_time attribute and may continue to use the value previously fetched (on the source server).

If state has not been transferred transparently (i.e., the client sees a real or simulated server reboot), the client should fetch the value of lease_time on the new (i.e., destination) server, and use it 
for subsequent locking requests. However the server must respect a grace period at least as long as the lease_time on the source server, in order to ensure that clients have ample time to reclaim their locks before potentially conflicting non-reclaimed locks are granted. The means by which the new server obtains the value of lease_time on the old server is left to the server implementations. It is not specified by the NFS version 4 protocol.

9. Client-Side Caching

Client-side caching of data, of file attributes, and of file names is essential to providing good performance with the NFS protocol. Providing distributed cache coherence is a difficult problem and previous versions of the NFS protocol have not attempted it. Instead, several NFS client implementation techniques have been used to reduce the problems that a lack of coherence poses for users. These techniques have not been clearly defined by earlier protocol specifications and it is often unclear what is valid or invalid client behavior.

The NFS version 4 protocol uses many techniques similar to those that have been used in previous protocol versions. The NFS version 4 protocol does not provide distributed cache coherence. However, it defines a more limited set of caching guarantees to allow locks and share reservations to be used without destructive interference from client side caching.

In addition, the NFS version 4 protocol introduces a delegation mechanism which allows many decisions normally made by the server to be made locally by clients. This mechanism provides efficient support of the common cases where sharing is infrequent or where sharing is read-only.

\subsection{Performance Challenges for Client-Side Caching}

Caching techniques used in previous versions of the NFS protocol have been successful in providing good performance. However, several scalability challenges can arise when those techniques are used with very large numbers of clients. This is particularly true when clients are geographically distributed which classically increases the latency for cache revalidation requests.

The previous versions of the NFS protocol repeat their file data cache validation requests at the time the file is opened. This behavior can have serious performance drawbacks. A common case is one in which a file is only accessed by a single client. Therefore, sharing is infrequent. 
In this case, repeated reference to the server to find that no conflicts exist is expensive. A better option with regards to performance is to allow a client that repeatedly opens a file to do so without reference to the server. This is done until potentially conflicting operations from another client actually occur.

A similar situation arises in connection with file locking. Sending file lock and unlock requests to the server as well as the read and write requests necessary to make data caching consistent with the locking semantics (see the section "Data Caching and File Locking") can severely limit performance. When locking is used to provide protection against infrequent conflicts, a large penalty is incurred. This penalty may discourage the use of file locking by applications.

The NFS version 4 protocol provides more aggressive caching strategies with the following design goals:

o Compatibility with a large range of server semantics.

- Provide the same caching benefits as previous versions of the NFS protocol when unable to provide the more aggressive model.

- Requirements for aggressive caching are organized so that a large portion of the benefit can be obtained even when not all of the requirements can be met.

The appropriate requirements for the server are discussed in later sections in which specific forms of caching are covered. (see the section "Open Delegation").

\subsection{Delegation and Callbacks}

Recallable delegation of server responsibilities for a file to a client improves performance by avoiding repeated requests to the server in the absence of inter-client conflict. With the use of a "callback" RPC from server to client, a server recalls delegated responsibilities when another client engages in sharing of a delegated file.

A delegation is passed from the server to the client, specifying the object of the delegation and the type of delegation. There are different types of delegations but each type contains a stateid to be used to represent the delegation when performing operations that depend on the delegation. This stateid is similar to those associated with locks and share reservations but differs in that the stateid for a delegation is associated with a clientid and may be 
used on behalf of all the open_owners for the given client. A delegation is made to the client as a whole and not to any specific process or thread of control within it.

Because callback RPCs may not work in all environments (due to firewalls, for example), correct protocol operation does not depend on them. Preliminary testing of callback functionality by means of a CB_NULL procedure determines whether callbacks can be supported. The CB_NULL procedure checks the continuity of the callback path. A server makes a preliminary assessment of callback availability to a given client and avoids delegating responsibilities until it has determined that callbacks are supported. Because the granting of a delegation is always conditional upon the absence of conflicting access, clients must not assume that a delegation will be granted and they must always be prepared for OPENs to be processed without any delegations being granted.

Once granted, a delegation behaves in most ways like a lock. There is an associated lease that is subject to renewal together with all of the other leases held by that client.

Unlike locks, an operation by a second client to a delegated file will cause the server to recall a delegation through a callback.

On recall, the client holding the delegation must flush modified state (such as modified data) to the server and return the delegation. The conflicting request will not receive a response until the recall is complete. The recall is considered complete when the client returns the delegation or the server times out on the recall and revokes the delegation as a result of the timeout. Following the resolution of the recall, the server has the information necessary to grant or deny the second client's request.

At the time the client receives a delegation recall, it may have substantial state that needs to be flushed to the server. Therefore, the server should allow sufficient time for the delegation to be returned since it may involve numerous RPCs to the server. If the server is able to determine that the client is diligently flushing state to the server as a result of the recall, the server may extend the usual time allowed for a recall. However, the time allowed for recall completion should not be unbounded.

An example of this is when responsibility to mediate opens on a given file is delegated to a client (see the section "Open Delegation"). The server will not know what opens are in effect on the client. Without this knowledge the server will be unable to determine if the access and deny state for the file allows any particular open until the delegation for the file has been returned. 
A client failure or a network partition can result in failure to respond to a recall callback. In this case, the server will revoke the delegation which in turn will render useless any modified state still on the client.

\subsubsection{Delegation Recovery}

There are three situations that delegation recovery must deal with:

o client reboot or restart

o Server reboot or restart

o Network partition (full or callback-only)

In the event the client reboots or restarts, the failure to renew leases will result in the revocation of record locks and share reservations. Delegations, however, may be treated a bit differently.

There will be situations in which delegations will need to be reestablished after a client reboots or restarts. The reason for this is the client may have file data stored locally and this data was associated with the previously held delegations. The client will need to reestablish the appropriate file state on the server.

To allow for this type of client recovery, the server MAY extend the period for delegation recovery beyond the typical lease expiration period. This implies that requests from other clients that conflict with these delegations will need to wait. Because the normal recall process may require significant time for the client to flush changed state to the server, other clients need be prepared for delays that occur because of a conflicting delegation. This longer interval would increase the window for clients to reboot and consult stable storage so that the delegations can be reclaimed. For open delegations, such delegations are reclaimed using OPEN with a claim type of CLAIM_DELEGATE_PREV. (See the sections on "Data Caching and Revocation" and "Operation 18: OPEN" for discussion of open delegation and the details of OPEN respectively).

A server MAY support a claim type of CLAIM_DELEGATE_PREV, but if it does, it MUST NOT remove delegations upon SETCLIENTID_CONFIRM, and instead MUST, for a period of time no less than that of the value of the lease_time attribute, maintain the client's delegations to allow time for the client to issue CLAIM_DELEGATE_PREV requests. The server that supports CLAIM_DELEGATE_PREV MUST support the DELEGPURGE operation. 
When the server reboots or restarts, delegations are reclaimed (using the OPEN operation with CLAIM_PREVIOUS) in a similar fashion to record locks and share reservations. However, there is a slight semantic difference. In the normal case if the server decides that a delegation should not be granted, it performs the requested action (e.g., OPEN) without granting any delegation. For reclaim, the server grants the delegation but a special designation is applied so that the client treats the delegation as having been granted but recalled by the server. Because of this, the client has the duty to write all modified state to the server and then return the delegation. This process of handling delegation reclaim reconciles three principles of the NFS version 4 protocol:

- Upon reclaim, a client reporting resources assigned to it by an earlier server instance must be granted those resources.

- The server has unquestionable authority to determine whether delegations are to be granted and, once granted, whether they are to be continued.

- The use of callbacks is not to be depended upon until the client has proven its ability to receive them.

When a network partition occurs, delegations are subject to freeing by the server when the lease renewal period expires. This is similar to the behavior for locks and share reservations. For delegations, however, the server may extend the period in which conflicting requests are held off. Eventually the occurrence of a conflicting request from another client will cause revocation of the delegation. A loss of the callback path (e.g., by later network configuration change) will have the same effect. A recall request will fail and revocation of the delegation will result.

A client normally finds out about revocation of a delegation when it uses a stateid associated with a delegation and receives the error NFS4ERR_EXPIRED. It also may find out about delegation revocation after a client reboot when it attempts to reclaim a delegation and receives that same error. Note that in the case of a revoked write open delegation, there are issues because data may have been modified by the client whose delegation is revoked and separately by other clients. See the section "Revocation Recovery for Write Open Delegation" for a discussion of such issues. Note also that when delegations are revoked, information about the revoked delegation will be written by the server to stable storage (as described in the section "Crash Recovery"). This is done to deal with the case in which a server reboots after revoking a delegation but before the client holding the revoked delegation is notified about the revocation. 


\subsection{Data Caching}

When applications share access to a set of files, they need to be implemented so as to take account of the possibility of conflicting access by another application. This is true whether the applications in question execute on different clients or reside on the same client.

Share reservations and record locks are the facilities the NFS version 4 protocol provides to allow applications to coordinate access by providing mutual exclusion facilities. The NFS version 4 protocol's data caching must be implemented such that it does not invalidate the assumptions that those using these facilities depend upon.

\subsubsection{Data Caching and OPENs}

In order to avoid invalidating the sharing assumptions that applications rely on, NFS version 4 clients should not provide cached data to applications or modify it on behalf of an application when it would not be valid to obtain or modify that same data via a READ or WRITE operation.

Furthermore, in the absence of open delegation (see the section "Open Delegation") two additional rules apply. Note that these rules are obeyed in practice by many NFS version 2 and version 3 clients.

- First, cached data present on a client must be revalidated after doing an OPEN. Revalidating means that the client fetches the change attribute from the server, compares it with the cached change attribute, and if different, declares the cached data (as well as the cached attributes) as invalid. This is to ensure that the data for the opENed file is still correctly reflected in the client's cache. This validation must be done at least when the client's OPEN operation includes DENY=WRITE or BOTH thus terminating a period in which other clients may have had the opportunity to open the file with WRITE access. Clients may choose to do the revalidation more often (i.e., at OPENs specifying DENY=NONE) to parallel the NFS version 3 protocol's practice for the benefit of users assuming this degree of cache revalidation.

Since the change attribute is updated for data and metadata modifications, some client implementors may be tempted to use the time_modify attribute and not change to validate cached data, so that metadata changes do not spuriously invalidate clean data. The implementor is cautioned in this approach. The change attribute is guaranteed to change for each update to the file, 
whereas time_modify is guaranteed to change only at the granularity of the time_delta attribute. Use by the client's data cache validation logic of time_modify and not change runs the risk of the client incorrectly marking stale data as valid.

- Second, modified data must be flushed to the server before closing a file opENed for write. This is complementary to the first rule. If the data is not flushed at CLOSE, the revalidation done after client OPENs as file is unable to achieve its purpose. The other aspect to flushing the data before close is that the data must be committed to stable storage, at the server, before the CLOSE operation is requested by the client. In the case of a server reboot or restart and a CLOSEd file, it may not be possible to retransmit the data to be written to the file. Hence, this requirement.

\subsubsection{Data Caching and File Locking}

For those applications that choose to use file locking instead of share reservations to exclude inconsistent file access, there is an analogous set of constraints that apply to client side data caching. These rules are effective only if the file locking is used in a way that matches in an equivalent way the actual READ and WRITE operations executed. This is as opposed to file locking that is based on pure convention. For example, it is possible to manipulate a two-megabyte file by dividing the file into two one-megabyte regions and protecting access to the two regions by file locks on bytes zero and one. A lock for write on byte zero of the file would represent the right to do READ and WRITE operations on the first region. A lock for write on byte one of the file would represent the right to do READ and WRITE operations on the second region. As long as all applications manipulating the file obey this convention, they will work on a local filesystem. However, they may not work with the NFS version 4 protocol unless clients refrain from data caching.

The rules for data caching in the file locking environment are:

- First, when a client obtains a file lock for a particular region, the data cache corresponding to that region (if any cached data exists) must be revalidated. If the change attribute indicates that the file may have been updated since the cached data was obtained, the client must flush or invalidate the cached data for the newly locked region. A client might choose to invalidate all of non-modified cached data that it has for the file but the only requirement for correct operation is to invalidate all of the data in the newly locked region. 
- Second, before releasing a write lock for a region, all modified data for that region must be flushed to the server. The modified data must also be written to stable storage.

Note that flushing data to the server and the invalidation of cached data must reflect the actual byte ranges locked or unlocked. Rounding these up or down to reflect client cache block boundaries will cause problems if not carefully done. For example, writing a modified block when only half of that block is within an area being unlocked may cause invalid modification to the region outside the unlocked area. This, in turn, may be part of a region locked by another client. Clients can avoid this situation by synchronously performing portions of write operations that overlap that portion (initial or final) that is not a full block. Similarly, invalidating a locked area which is not an integral number of full buffer blocks would require the client to read one or two partial blocks from the server if the revalidation procedure shows that the data which the client possesses may not be valid.

The data that is written to the server as a prerequisite to the unlocking of a region must be written, at the server, to stable storage. The client may accomplish this either with synchronous writes or by following asynchronous writes with a COMMIT operation. This is required because retransmission of the modified data after a server reboot might conflict with a lock held by another client.

A client implementation may choose to accommodate applications which use record locking in non-standard ways (e.g., using a record lock as a global semaphore) by flushing to the server more data upon an LOCKU than is covered by the locked range. This may include modified data within files other than the one for which the unlocks are being done. In such cases, the client must not interfere with applications whose READs and WRITEs are being done only within the bounds of record locks which the application holds. For example, an application locks a single byte of a file and proceeds to write that single byte. A client that chose to handle a LOCKU by flushing all modified data to the server could validly write that single byte in response to an unrelated unlock. However, it would not be valid to write the entire block in which that single written byte was located since it includes an area that is not locked and might be locked by another client. Client implementations can avoid this problem by dividing files with modified data into those for which all modifications are done to areas covered by an appropriate record lock and those for which there are modifications not covered by a record lock. Any writes done for the former class of files must not include areas not locked and thus not modified on the client. 


\subsubsection{Data Caching and Mandatory File Locking}

Client side data caching needs to respect mandatory file locking when it is in effect. The presence of mandatory file locking for a given file is indicated when the client gets back NFS4ERR_LOCKED from a READ or WRITE on a file it has an appropriate share reservation for. When mandatory locking is in effect for a file, the client must check for an appropriate file lock for data being read or written. If a lock exists for the range being read or written, the client may satisfy the request using the client's validated cache. If an appropriate file lock is not held for the range of the read or write, the read or write request must not be satisfied by the client's cache and the request must be sent to the server for processing. When a read or write request partially overlaps a locked region, the request should be subdivided into multiple pieces with each region (locked or not) treated appropriately.

\subsubsection{Data Caching and File Identity}

When clients cache data, the file data needs to be organized according to the filesystem object to which the data belongs. For NFS version 3 clients, the typical practice has been to assume for the purpose of caching that distinct filehandles represent distinct filesystem objects. The client then has the choice to organize and maintain the data cache on this basis.

In the NFS version 4 protocol, there is now the possibility to have significant deviations from a "one filehandle per object" model because a filehandle may be constructed on the basis of the object's pathname. Therefore, clients need a reliable method to determine if two filehandles designate the same filesystem object. If clients were simply to assume that all distinct filehandles denote distinct objects and proceed to do data caching on this basis, caching inconsistencies would arise between the distinct client side objects which mapped to the same server side object.

By providing a method to differentiate filehandles, the NFS version 4 protocol alleviates a potential functional regression in comparison with the NFS version 3 protocol. Without this method, caching inconsistencies within the same client could occur and this has not been present in previous versions of the NFS protocol. Note that it is possible to have such inconsistencies with applications executing on multiple clients but that is not the issue being addressed here.

For the purposes of data caching, the following steps allow an NFS version 4 client to determine whether two distinct filehandles denote the same server side object: 
- If GETATTR directed to two filehandles returns different values of the fsid attribute, then the filehandles represent distinct objects.

- If GETATTR for any file with an fsid that matches the fsid of the two filehandles in question returns a unique_handles attribute with a value of TRUE, then the two objects are distinct.

- If GETATTR directed to the two filehandles does not return the fileid attribute for both of the handles, then it cannot be determined whether the two objects are the same. Therefore, operations which depend on that knowledge (e.g., client side data caching) cannot be done reliably.

- If GETATTR directed to the two filehandles returns different values for the fileid attribute, then they are distinct objects.

o Otherwise they are the same object.

9.4. Open Delegation

When a file is being OPENed, the server may delegate further handling of opens and closes for that file to the opening client. Any such delegation is recallable, since the circumstances that allowed for the delegation are subject to change. In particular, the server may receive a conflicting OPEN from another client, the server must recall the delegation before deciding whether the OPEN from the other client may be granted. Making a delegation is up to the server and clients should not assume that any particular OPEN either will or will not result in an open delegation. The following is a typical set of conditions that servers might use in deciding whether OPEN should be delegated:

- The client must be able to respond to the server's callback requests. The server will use the CB_NULL procedure for a test of callback ability.

- The client must have responded properly to previous recalls.

- There must be no current open conflicting with the requested delegation.

- There should be no current delegation that conflicts with the delegation being requested.

- The probability of future conflicting open requests should be low based on the recent history of the file. 
- The existence of any server-specific semantics of OPEN/CLOSE that would make the required handling incompatible with the prescribed handling that the delegated client would apply (see below).

There are two types of open delegations, read and write. A read open delegation allows a client to handle, on its own, requests to open a file for reading that do not deny read access to others. Multiple read open delegations may be outstanding simultaneously and do not conflict. A write open delegation allows the client to handle, on its own, all opens. Only one write open delegation may exist for a given file at a given time and it is inconsistent with any read open delegations.

When a client has a read open delegation, it may not make any changes to the contents or attributes of the file but it is assured that no other client may do so. When a client has a write open delegation, it may modify the file data since no other client will be accessing the file's data. The client holding a write delegation may only affect file attributes which are intimately connected with the file data: size, time_modify, change.

When a client has an open delegation, it does not send OPENs or CLOSEs to the server but updates the appropriate status internally. For a read open delegation, opens that cannot be handled locally (opens for write or that deny read access) must be sent to the server.

When an open delegation is made, the response to the OPEN contains an open delegation structure which specifies the following:

- the type of delegation (read or write)

- space limitation information to control flushing of data on close (write open delegation only, see the section "Open Delegation and Data Caching")

o an nfsace4 specifying read and write permissions

- a stateid to represent the delegation for READ and WRITE

The delegation stateid is separate and distinct from the stateid for the OPEN proper. The standard stateid, unlike the delegation stateid, is associated with a particular lock_owner and will continue to be valid after the delegation is recalled and the file remains open. 
When a request internal to the client is made to open a file and open delegation is in effect, it will be accepted or rejected solely on the basis of the following conditions. Any requirement for other checks to be made by the delegate should result in open delegation being denied so that the checks can be made by the server itself.

- The access and deny bits for the request and the file as described in the section "Share Reservations".

- The read and write permissions as determined below.

The nfsace4 passed with delegation can be used to avoid frequent ACCESS calls. The permission check should be as follows:

- If the nfsace4 indicates that the open may be done, then it should be granted without reference to the server.

- If the nfsace4 indicates that the open may not be done, then an ACCESS request must be sent to the server to obtain the definitive answer.

The server may return an nfsace4 that is more restrictive than the actual ACL of the file. This includes an nfsace4 that specifies denial of all access. Note that some common practices such as mapping the traditional user "root" to the user "nobody" may make it incorrect to return the actual ACL of the file in the delegation response.

The use of delegation together with various other forms of caching creates the possibility that no server authentication will ever be performed for a given user since all of the user's requests might be satisfied locally. Where the client is depending on the server for authentication, the client should be sure authentication occurs for each user by use of the ACCESS operation. This should be the case even if an ACCESS operation would not be required otherwise. As mentioned before, the server may enforce frequent authentication by returning an nfsace4 denying all access with every open delegation.

\subsubsection{Open Delegation and Data Caching}

OPEN delegation allows much of the message overhead associated with the opening and closing files to be eliminated. An open when an open delegation is in effect does not require that a validation message be sent to the server. The continued endurance of the "read open delegation" provides a guarantee that no OPEN for write and thus no write has occurred. Similarly, when closing a file opened for write and if write open delegation is in effect, the data written does not have to be flushed to the server until the open delegation is 
recalled. The continued endurance of the open delegation provides a guarantee that no open and thus no read or write has been done by another client.

For the purposes of open delegation, READs and WRITEs done without an OPEN are treated as the functional equivalents of a corresponding type of OPEN. This refers to the READs and WRITEs that use the special stateids consisting of all zero bits or all one bits. Therefore, READs or WRITEs with a special stateid done by another client will force the server to recall a write open delegation. A WRITE with a special stateid done by another client will force a recall of read open delegations.

With delegations, a client is able to avoid writing data to the server when the CLOSE of a file is serviced. The file close system call is the usual point at which the client is notified of a lack of stable storage for the modified file data generated by the application. At the close, file data is written to the server and through normal accounting the server is able to determine if the available filesystem space for the data has been exceeded (i.e., server returns NFS4ERR_NOSPC or NFS4ERR_DQUOT). This accounting includes quotas. The introduction of delegations requires that a alternative method be in place for the same type of communication to occur between client and server.

In the delegation response, the server provides either the limit of the size of the file or the number of modified blocks and associated block size. The server must ensure that the client will be able to flush data to the server of a size equal to that provided in the original delegation. The server must make this assurance for all outstanding delegations. Therefore, the server must be careful in its management of available space for new or modified data taking into account available filesystem space and any applicable quotas. The server can recall delegations as a result of managing the available filesystem space. The client should abide by the server's state space limits for delegations. If the client exceeds the stated limits for the delegation, the server's behavior is undefined.

Based on server conditions, quotas or available filesystem space, the server may grant write open delegations with very restrictive space limitations. The limitations may be defined in a way that will always force modified data to be flushed to the server on close.

With respect to authentication, flushing modified data to the server after a CLOSE has occurred may be problematic. For example, the user of the application may have logged off the client and unexpired authentication credentials may not be present. In this case, the client may need to take special care to ensure that local unexpired 
credentials will in fact be available. This may be accomplished by tracking the expiration time of credentials and flushing data well in advance of their expiration or by making private copies of credentials to assure their availability when needed.

\subsubsection{Open Delegation and File Locks}

When a client holds a write open delegation, lock operations may be performed locally. This includes those required for mandatory file locking. This can be done since the delegation implies that there can be no conflicting locks. Similarly, all of the revalidations that would normally be associated with obtaining locks and the flushing of data associated with the releasing of locks need not be done.

When a client holds a read open delegation, lock operations are not performed locally. All lock operations, including those requesting non-exclusive locks, are sent to the server for resolution.

\subsubsection{Handling of CB_GETATTR}

The server needs to employ special handling for a GETATTR where the target is a file that has a write open delegation in effect. The reason for this is that the client holding the write delegation may have modified the data and the server needs to reflect this change to the second client that submitted the GETATTR. Therefore, the client holding the write delegation needs to be interrogated. The server will use the CB_GETATTR operation. The only attributes that the server can reliably query via CB_GETATTR are size and change.

Since CB_GETATTR is being used to satisfy another client's GETATTR request, the server only needs to know if the client holding the delegation has a modified version of the file. If the client's copy of the delegated file is not modified (data or size), the server can satisfy the second client's GETATTR request from the attributes stored locally at the server. If the file is modified, the server only needs to know about this modified state. If the server determines that the file is currently modified, it will respond to the second client's GETATTR as if the file had been modified locally at the server.

Since the form of the change attribute is determined by the server and is opaque to the client, the client and server need to agree on a method of communicating the modified state of the file. For the size attribute, the client will report its current view of the file size.

For the change attribute, the handling is more involved. 
For the client, the following steps will be taken when receiving a write delegation:

- The value of the change attribute will be obtained from the server and cached. Let this value be represented by $c$.

- The client will create a value greater than c that will be used for communicating modified data is held at the client. Let this value be represented by $d$.

- When the client is queried via CB_GETATTR for the change attribute, it checks to see if it holds modified data. If the file is modified, the value d is returned for the change attribute value. If this file is not currently modified, the client returns the value $\mathrm{c}$ for the change attribute.

For simplicity of implementation, the client MAY for each CB_GETATTR return the same value $d$. This is true even if, between successive CB_GETATTR operations, the client again modifies in the file's data or metadata in its cache. The client can return the same value because the only requirement is that the client be able to indicate to the server that the client holds modified data. Therefore, the value of d may always be $\mathrm{c}+1$.

While the change attribute is opaque to the client in the sense that it has no idea what units of time, if any, the server is counting change with, it is not opaque in that the client has to treat it as an unsigned integer, and the server has to be able to see the results of the client's changes to that integer. Therefore, the server MUST encode the change attribute in network order when sending it to the client. The client MUST decode it from network order to its native order when receiving it and the client MUST encode it network order when sending it to the server. For this reason, change is defined as an unsigned integer rather than an opaque array of octets.

For the server, the following steps will be taken when providing a write delegation:

- Upon providing a write delegation, the server will cache a copy of the change attribute in the data structure it uses to record the delegation. Let this value be represented by sc.

- When a second client sends a GETATTR operation on the same file to the server, the server obtains the change attribute from the first client. Let this value be cc. 
- If the value $\mathrm{cc}$ is equal to sc, the file is not modified and the server returns the current values for change, time_metadata, and time_modify (for example) to the second client.

- If the value $\mathrm{cc}$ is NOT equal to sc, the file is currently modified at the first client and most likely will be modified at the server at a future time. The server then uses its current time to construct attribute values for time_metadata and time_modify. A new value of $\mathrm{sc}$, which we will call nsc, is computed by the server, such that $\mathrm{nsc}>=\mathrm{sc}+1$. The server then returns the constructed time_metadata, time_modify, and nsc values to the requester. The server replaces $\mathrm{sc}$ in the delegation record with nsc. To prevent the possibility of time_modify, time_metadata, and change from appearing to go backward (which would happen if the client holding the delegation fails to write its modified data to the server before the delegation is revoked or returned), the server SHOULD update the file's metadata record with the constructed attribute values. For reasons of reasonable performance, committing the constructed attribute values to stable storage is OPTIONAL.

As discussed earlier in this section, the client MAY return the same CC value on subsequent CB_GETATTR calls, even if the file was modified in the client's cache yet again between successive CB_GETATTR calls. Therefore, the server must assume that the file has been modified yet again, and MUST take care to ensure that the new nsc it constructs and returns is greater than the previous nsc it returned. An example implementation's delegation record would satisfy this mandate by including a boolean field (let us call it "modified") that is set to false when the delegation is granted, and an sc value set at the time of grant to the change attribute value. The modified field would be set to true the first time cc != sc, and would stay true until the delegation is returned or revoked. The processing for constructing nsc, time_modify, and time_metadata would use this pseudo code:

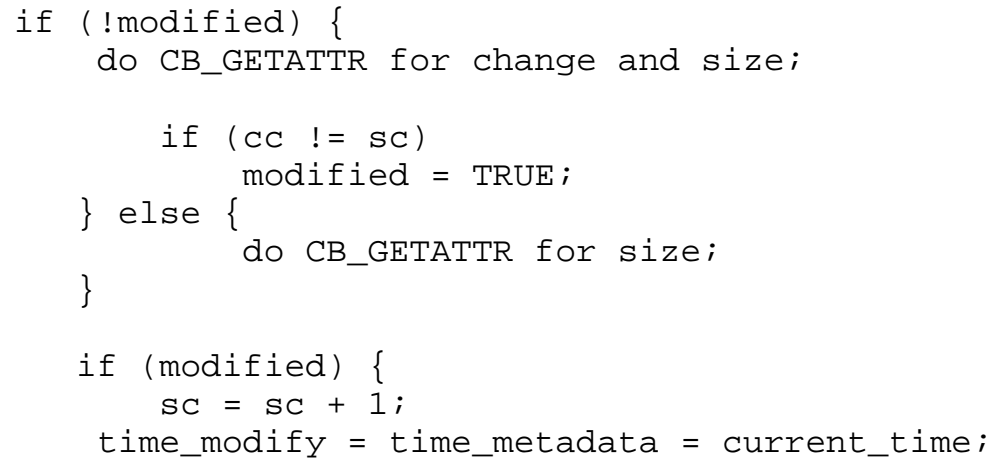


- In the case that the file attribute size is different than the server's current value, the server treats this as a modification regardless of the value of the change attribute retrieved via CB_GETATTR and responds to the second client as in the last step.

This methodology resolves issues of clock differences between client and server and other scenarios where the use of CB_GETATTR break down.

It should be noted that the server is under no obligation to use CB_GETATTR and therefore the server MAY simply recall the delegation to avoid its use.

\subsubsection{Recall of Open Delegation}

The following events necessitate recall of an open delegation:

- Potentially conflicting OPEN request (or READ/WRITE done with "special" stateid)

- SETATTR issued by another client

- REMOVE request for the file

- RENAME request for the file as either source or target of the RENAME

Whether a RENAME of a directory in the path leading to the file results in recall of an open delegation depends on the semantics of the server filesystem. If that filesystem denies such RENAMEs when a file is open, the recall must be performed to determine whether the file in question is, in fact, open.

In addition to the situations above, the server may choose to recall open delegations at any time if resource constraints make it advisable to do so. Clients should always be prepared for the possibility of recall. 
When a client receives a recall for an open delegation, it needs to update state on the server before returning the delegation. These same updates must be done whenever a client chooses to return a delegation voluntarily. The following items of state need to be dealt with:

- If the file associated with the delegation is no longer open and no previous CLOSE operation has been sent to the server, a CLOSE operation must be sent to the server.

- If a file has other open references at the client, then OPEN operations must be sent to the server. The appropriate stateids will be provided by the server for subsequent use by the client since the delegation stateid will not longer be valid. These OPEN requests are done with the claim type of CLAIM_DELEGATE_CUR. This will allow the presentation of the delegation stateid so that the client can establish the appropriate rights to perform the OPEN. (see the section "Operation 18: OPEN" for details.)

- If there are granted file locks, the corresponding Lock operations need to be performed. This applies to the write open delegation case only.

- For a write open delegation, if at the time of recall the file is not open for write, all modified data for the file must be flushed to the server. If the delegation had not existed, the client would have done this data flush before the CLOSE operation.

- For a write open delegation when a file is still open at the time of recall, any modified data for the file needs to be flushed to the server.

- With the write open delegation in place, it is possible that the file was truncated during the duration of the delegation. For example, the truncation could have occurred as a result of an OPEN UNCHECKED with a size attribute value of zero. Therefore, if a truncation of the file has occurred and this operation has not been propagated to the server, the truncation must occur before any modified data is written to the server.

In the case of write open delegation, file locking imposes some additional requirements. To precisely maintain the associated invariant, it is required to flush any modified data in any region for which a write lock was released while the write delegation was in effect. However, because the write open delegation implies no other locking by other clients, a simpler implementation is to flush all modified data for the file (as described just above) if any write lock has been released while the write open delegation was in effect. 
An implementation need not wait until delegation recall (or deciding to voluntarily return a delegation) to perform any of the above actions, if implementation considerations (e.g., resource availability constraints) make that desirable. Generally, however, the fact that the actual open state of the file may continue to change makes it not worthwhile to send information about opens and closes to the server, except as part of delegation return. Only in the case of closing the open that resulted in obtaining the delegation would clients be likely to do this early, since, in that case, the close once done will not be undone. Regardless of the client's choices on scheduling these actions, all must be performed before the delegation is returned, including (when applicable) the close that corresponds to the open that resulted in the delegation. These actions can be performed either in previous requests or in previous operations in the same COMPOUND request.

\subsubsection{Clients that Fail to Honor Delegation Recalls}

A client may fail to respond to a recall for various reasons, such as a failure of the callback path from server to the client. The client may be unaware of a failure in the callback path. This lack of awareness could result in the client finding out long after the failure that its delegation has been revoked, and another client has modified the data for which the client had a delegation. This is especially a problem for the client that held a write delegation.

The server also has a dilemma in that the client that fails to respond to the recall might also be sending other NFS requests, including those that renew the lease before the lease expires. Without returning an error for those lease renewing operations, the server leads the client to believe that the delegation it has is in force.

This difficulty is solved by the following rules:

- When the callback path is down, the server MUST NOT revoke the delegation if one of the following occurs:

- The client has issued a RENEW operation and the server has returned an NFS4ERR_CB_PATH_DOWN error. The server MUST renew the lease for any record locks and share reservations the client has that the server has known about (as opposed to those locks and share reservations the client has established but not yet sent to the server, due to the delegation). The server SHOULD give the client a reasonable time to return its delegations to the server before revoking the client's delegations. 
- The client has not issued a RENEW operation for some period of time after the server attempted to recall the delegation. This period of time MUST NOT be less than the value of the lease_time attribute.

- When the client holds a delegation, it can not rely on operations, except for RENEW, that take a stateid, to renew delegation leases across callback path failures. The client that wants to keep delegations in force across callback path failures must use RENEW to do so.

\subsubsection{Delegation Revocation}

At the point a delegation is revoked, if there are associated opens on the client, the applications holding these opens need to be notified. This notification usually occurs by returning errors for READ/WRITE operations or when a close is attempted for the open file.

If no opens exist for the file at the point the delegation is revoked, then notification of the revocation is unnecessary. However, if there is modified data present at the client for the file, the user of the application should be notified. Unfortunately, it may not be possible to notify the user since active applications may not be present at the client. See the section "Revocation Recovery for Write Open Delegation" for additional details.

\subsection{Data Caching and Revocation}

When locks and delegations are revoked, the assumptions upon which successful caching depend are no longer guaranteed. For any locks or share reservations that have been revoked, the corresponding owner needs to be notified. This notification includes applications with a file open that has a corresponding delegation which has been revoked. Cached data associated with the revocation must be removed from the client. In the case of modified data existing in the client's cache, that data must be removed from the client without it being written to the server. As mentioned, the assumptions made by the client are no longer valid at the point when a lock or delegation has been revoked. For example, another client may have been granted a conflicting lock after the revocation of the lock at the first client. Therefore, the data within the lock range may have been modified by the other client. Obviously, the first client is unable to guarantee to the application what has occurred to the file in the case of revocation.

Notification to a lock owner will in many cases consist of simply returning an error on the next and all subsequent READs/WRITEs to the open file or on the close. Where the methods available to a client make such notification impossible because errors for certain 
operations may not be returned, more drastic action such as signals or process termination may be appropriate. The justification for this is that an invariant for which an application depends on may be violated. Depending on how errors are typically treated for the client operating environment, further levels of notification including logging, console messages, and GUI pop-ups may be appropriate.

\subsubsection{Revocation Recovery for Write Open Delegation}

Revocation recovery for a write open delegation poses the special issue of modified data in the client cache while the file is not open. In this situation, any client which does not flush modified data to the server on each close must ensure that the user receives appropriate notification of the failure as a result of the revocation. Since such situations may require human action to correct problems, notification schemes in which the appropriate user or administrator is notified may be necessary. Logging and console messages are typical examples.

If there is modified data on the client, it must not be flushed normally to the server. A client may attempt to provide a copy of the file data as modified during the delegation under a different name in the filesystem name space to ease recovery. Note that when the client can determine that the file has not been modified by any other client, or when the client has a complete cached copy of file in question, such a saved copy of the client's view of the file may be of particular value for recovery. In other case, recovery using a copy of the file based partially on the client's cached data and partially on the server copy as modified by other clients, will be anything but straightforward, so clients may avoid saving file contents in these situations or mark the results specially to warn users of possible problems.

Saving of such modified data in delegation revocation situations may be limited to files of a certain size or might be used only when sufficient disk space is available within the target filesystem. Such saving may also be restricted to situations when the client has sufficient buffering resources to keep the cached copy available until it is properly stored to the target filesystem.

\subsection{Attribute Caching}

The attributes discussed in this section do not include named attributes. Individual named attributes are analogous to files and caching of the data for these needs to be handled just as data 
caching is for ordinary files. Similarly, LookUP results from an OPENATTR directory are to be cached on the same basis as any other pathnames and similarly for directory contents.

Clients may cache file attributes obtained from the server and use them to avoid subsequent GETATTR requests. Such caching is write through in that modification to file attributes is always done by means of requests to the server and should not be done locally and cached. The exception to this are modifications to attributes that are intimately connected with data caching. Therefore, extending a file by writing data to the local data cache is reflected immediately in the size as seen on the client without this change being immediately reflected on the server. Normally such changes are not propagated directly to the server but when the modified data is flushed to the server, analogous attribute changes are made on the server. When open delegation is in effect, the modified attributes may be returned to the server in the response to a CB_RECALL call.

The result of local caching of attributes is that the attribute caches maintained on individual clients will not be coherent. Changes made in one order on the server may be seen in a different order on one client and in a third order on a different client.

The typical filesystem application programming interfaces do not provide means to atomically modify or interrogate attributes for multiple files at the same time. The following rules provide an environment where the potential incoherences mentioned above can be reasonably managed. These rules are derived from the practice of previous NFS protocols.

- All attributes for a given file (per-fsid attributes excepted) are cached as a unit at the client so that no non-serializability can arise within the context of a single file.

- An upper time boundary is maintained on how long a client cache entry can be kept without being refreshed from the server.

- When operations are performed that change attributes at the server, the updated attribute set is requested as part of the containing RPC. This includes directory operations that update attributes indirectly. This is accomplished by following the modifying operation with a GETATTR operation and then using the results of the GETATTR to update the client's cached attributes.

Note that if the full set of attributes to be cached is requested by READDIR, the results can be cached by the client on the same basis as attributes obtained via GETATTR. 
A client may validate its cached version of attributes for a file by fetching just both the change and time_access attributes and assuming that if the change attribute has the same value as it did when the attributes were cached, then no attributes other than time_access have changed. The reason why time_access is also fetched is because many servers operate in environments where the operation that updates change does not update time_access. For example, Posix file semantics do not update access time when a file is modified by the write system call. Therefore, the client that wants a current time_access value should fetch it with change during the attribute cache validation processing and update its cached time_access.

The client may maintain a cache of modified attributes for those attributes intimately connected with data of modified regular files (size, time_modify, and change). Other than those three attributes, the client MUST NOT maintain a cache of modified attributes. Instead, attribute changes are immediately sent to the server.

In some operating environments, the equivalent to time_access is expected to be implicitly updated by each read of the content of the file object. If an NFS client is caching the content of a file object, whether it is a regular file, directory, or symbolic link, the client SHOULD NOT update the time_access attribute (via SETATTR or a small READ or READDIR request) on the server with each read that is satisfied from cache. The reason is that this can defeat the performance benefits of caching content, especially since an explicit SETATTR of time_access may alter the change attribute on the server. If the change attribute changes, clients that are caching the content will think the content has changed, and will re-read unmodified data from the server. Nor is the client encouraged to maintain a modified version of time_access in its cache, since this would mean that the client will either eventually have to write the access time to the server with bad performance effects, or it would never update the server's time_access, thereby resulting in a situation where an application that caches access time between a close and open of the same file observes the access time oscillating between the past and present. The time_access attribute always means the time of last access to a file by a read that was satisfied by the server. This way clients will tend to see only time_access changes that go forward in time.

\subsection{Data and Metadata Caching and Memory Mapped Files}

Some operating environments include the capability for an application to map a file's content into the application's address space. Each time the application accesses a memory location that corresponds to a block that has not been loaded into the address space, a page fault occurs and the file is read (or if the block does not exist in the 
file, the block is allocated and then instantiated in the application's address space).

As long as each memory mapped access to the file requires a page fault, the relevant attributes of the file that are used to detect access and modification (time_access, time_metadata, time_modify, and change) will be updated. However, in many operating environments, when page faults are not required these attributes will not be updated on reads or updates to the file via memory access (regardless whether the file is local file or is being access remotely). A client or server MAY fail to update attributes of a file that is being accessed via memory mapped I/O. This has several implications:

- If there is an application on the server that has memory mapped a file that a client is also accessing, the client may not be able to get a consistent value of the change attribute to determine whether its cache is stale or not. A server that knows that the file is memory mapped could always pessimistically return updated values for change so as to force the application to always get the most up to date data and metadata for the file. However, due to the negative performance implications of this, such behavior is OPTIONAL .

- If the memory mapped file is not being modified on the server, and instead is just being read by an application via the memory mapped interface, the client will not see an updated time_access attribute. However, in many operating environments, neither will any process running on the server. Thus NFS clients are at no disadvantage with respect to local processes.

- If there is another client that is memory mapping the file, and if that client is holding a write delegation, the same set of issues as discussed in the previous two bullet items apply. So, when a server does a CB_GETATTR to a file that the client has modified in its cache, the response from CB_GETATTR will not necessarily be accurate. As discussed earlier, the client's obligation is to report that the file has been modified since the delegation was granted, not whether it has been modified again between successive CB_GETATTR calls, and the server MUST assume that any file the client has modified in cache has been modified again between successive CB_GETATTR calls. Depending on the nature of the client's memory management system, this weak obligation may not be possible. A client MAY return stale information in CB_GETATTR whenever the file is memory mapped.

- The mixture of memory mapping and file locking on the same file is problematic. Consider the following scenario, where the page size on each client is 8192 bytes. 
- Client A memory maps first page (8192 bytes) of file X

- Client B memory maps first page (8192 bytes) of file X

- Client A write locks first 4096 bytes

- Client B write locks second 4096 bytes

- Client A, via a STORE instruction modifies part of its locked region.

- Simultaneous to client A, client B issues a STORE on part of its locked region.

Here the challenge is for each client to resynchronize to get a correct view of the first page. In many operating environments, the virtual memory management systems on each client only know a page is modified, not that a subset of the page corresponding to the respective lock regions has been modified. So it is not possible for each client to do the right thing, which is to only write to the server that portion of the page that is locked. For example, if client A simply writes out the page, and then client B writes out the page, client $A^{\prime}$ s data is lost.

Moreover, if mandatory locking is enabled on the file, then we have a different problem. When clients A and B issue the STORE instructions, the resulting page faults require a record lock on the entire page. Each client then tries to extend their locked range to the entire page, which results in a deadlock.

Communicating the NFS4ERR_DEADLOCK error to a STORE instruction is difficult at best.

If a client is locking the entire memory mapped file, there is no problem with advisory or mandatory record locking, at least until the client unlocks a region in the middle of the file.

Given the above issues the following are permitted:

- Clients and servers MAY deny memory mapping a file they know there are record locks for.

- Clients and servers MAY deny a record lock on a file they know is memory mapped. 
- A client MAY deny memory mapping a file that it knows requires mandatory locking for I/O. If mandatory locking is enabled after the file is opened and mapped, the client MAY deny the application further access to its mapped file.

\subsection{Name Caching}

The results of LOOKUP and READDIR operations may be cached to avoid the cost of subsequent LOOKUP operations. Just as in the case of attribute caching, inconsistencies may arise among the various client caches. To mitigate the effects of these inconsistencies and given the context of typical filesystem APIs, an upper time boundary is maintained on how long a client name cache entry can be kept without verifying that the entry has not been made invalid by a directory change operation performed by another client.

When a client is not making changes to a directory for which there exist name cache entries, the client needs to periodically fetch attributes for that directory to ensure that it is not being modified. After determining that no modification has occurred, the expiration time for the associated name cache entries may be updated to be the current time plus the name cache staleness bound.

When a client is making changes to a given directory, it needs to determine whether there have been changes made to the directory by other clients. It does this by using the change attribute as reported before and after the directory operation in the associated change_info4 value returned for the operation. The server is able to communicate to the client whether the change_info4 data is provided atomically with respect to the directory operation. If the change values are provided atomically, the client is then able to compare the pre-operation change value with the change value in the client's name cache. If the comparison indicates that the directory was updated by another client, the name cache associated with the modified directory is purged from the client. If the comparison indicates no modification, the name cache can be updated on the client to reflect the directory operation and the associated timeout extended. The post-operation change value needs to be saved as the basis for future change_info4 comparisons.

As demonstrated by the scenario above, name caching requires that the client revalidate name cache data by inspecting the change attribute of a directory at the point when the name cache item was cached. This requires that the server update the change attribute for directories when the contents of the corresponding directory is modified. For a client to use the change_info4 information appropriately and correctly, the server must report the pre and post operation change attribute values atomically. When the server is 
unable to report the before and after values atomically with respect to the directory operation, the server must indicate that fact in the change_info4 return value. When the information is not atomically reported, the client should not assume that other clients have not changed the directory.

\subsection{Directory Caching}

The results of READDIR operations may be used to avoid subsequent READDIR operations. Just as in the cases of attribute and name caching, inconsistencies may arise among the various client caches. To mitigate the effects of these inconsistencies, and given the context of typical filesystem APIs, the following rules should be followed:

- Cached READDIR information for a directory which is not obtained in a single READDIR operation must always be a consistent snapshot of directory contents. This is determined by using a GETATTR before the first READDIR and after the last of READDIR that contributes to the cache.

- An upper time boundary is maintained to indicate the length of time a directory cache entry is considered valid before the client must revalidate the cached information.

The revalidation technique parallels that discussed in the case of name caching. When the client is not changing the directory in question, checking the change attribute of the directory with GETATTR is adequate. The lifetime of the cache entry can be extended at these checkpoints. When a client is modifying the directory, the client needs to use the change_info4 data to determine whether there are other clients modifying the directory. If it is determined that no other client modifications are occurring, the client may update its directory cache to reflect its own changes.

As demonstrated previously, directory caching requires that the client revalidate directory cache data by inspecting the change attribute of a directory at the point when the directory was cached. This requires that the server update the change attribute for directories when the contents of the corresponding directory is modified. For a client to use the change_info4 information appropriately and correctly, the server must report the pre and post operation change attribute values atomically. When the server is unable to report the before and after values atomically with respect to the directory operation, the server must indicate that fact in the change_info4 return value. When the information is not atomically reported, the client should not assume that other clients have not changed the directory. 


\section{Minor Versioning}

To address the requirement of an NFS protocol that can evolve as the need arises, the NFS version 4 protocol contains the rules and framework to allow for future minor changes or versioning.

The base assumption with respect to minor versioning is that any future accepted minor version must follow the IETF process and be documented in a standards track RFC. Therefore, each minor version number will correspond to an RFC. Minor version zero of the NFS version 4 protocol is represented by this RFC. The COMPOUND procedure will support the encoding of the minor version being requested by the client.

The following items represent the basic rules for the development of minor versions. Note that a future minor version may decide to modify or add to the following rules as part of the minor version definition.

1. Procedures are not added or deleted

To maintain the general RPC model, NFS version 4 minor versions will not add to or delete procedures from the NFS program.

2. Minor versions may add operations to the COMPOUND and CB_COMPOUND procedures.

The addition of operations to the COMPOUND and CB_COMPOUND procedures does not affect the RPC model.

2.1 Minor versions may append attributes to GETATTR4args, bitmap4, and GETATTR4res.

This allows for the expansion of the attribute model to allow for future growth or adaptation.

2.2 Minor version X must append any new attributes after the last documented attribute.

Since attribute results are specified as an opaque array of per-attribute XDR encoded results, the complexity of adding new attributes in the midst of the current definitions will be too burdensome.

3. Minor versions must not modify the structure of an existing operation's arguments or results. 
Again the complexity of handling multiple structure definitions for a single operation is too burdensome. New operations should be added instead of modifying existing structures for a minor version.

This rule does not preclude the following adaptations in a minor version.

- adding bits to flag fields such as new attributes to GETATTR's bitmap4 data type

- adding bits to existing attributes like ACLs that have flag words

- extending enumerated types (including NFS4ERR_*) with new values

4. Minor versions may not modify the structure of existing attributes.

5. Minor versions may not delete operations.

This prevents the potential reuse of a particular operation "slot" in a future minor version.

6. Minor versions may not delete attributes.

7. Minor versions may not delete flag bits or enumeration values.

8. Minor versions may declare an operation as mandatory to NOT implement.

Specifying an operation as "mandatory to not implement" is equivalent to obsoleting an operation. For the client, it means that the operation should not be sent to the server. For the server, an NFS error can be returned as opposed to "dropping" the request as an XDR decode error. This approach allows for the obsolescence of an operation while maintaining its structure so that a future minor version can reintroduce the operation.

8.1 Minor versions may declare attributes mandatory to NOT implement.

8.2 Minor versions may declare flag bits or enumeration values as mandatory to NOT implement.

9. Minor versions may downgrade features from mandatory to recommended, or recommended to optional. 
10. Minor versions may upgrade features from optional to recommended or recommended to mandatory.

11. A client and server that support minor version $\mathrm{X}$ must support minor versions 0 (zero) through $\mathrm{X}-1$ as well.

12. No new features may be introduced as mandatory in a minor version.

This rule allows for the introduction of new functionality and forces the use of implementation experience before designating a feature as mandatory.

13. A client MUST NOT attempt to use a stateid, filehandle, or similar returned object from the COMPOUND procedure with minor version $\mathrm{X}$ for another COMPOUND procedure with minor version $\mathrm{Y}$, where $\mathrm{X} !=\mathrm{Y}$.

\title{
11. Internationalization
}

\begin{abstract}
The primary issue in which NFS version 4 needs to deal with internationalization, or I $18 \mathrm{~N}$, is with respect to file names and other strings as used within the protocol. The choice of string representation must allow reasonable name/string access to clients which use various languages. The UTF-8 encoding of the UCS as defined by [ISO10646] allows for this type of access and follows the policy described in "IETF Policy on Character sets and Languages", [RFC2277].
\end{abstract}

[RFC3454], otherwise know as "stringprep", documents a framework for using Unicode/UTF-8 in networking protocols, so as "to increase the likelihood that string input and string comparison work in ways that make sense for typical users throughout the world." A protocol must define a profile of stringprep "in order to fully specify the processing options." The remainder of this Internationalization section defines the NFS version 4 stringprep profiles. Much of terminology used for the remainder of this section comes from stringprep.

There are three UTF-8 string types defined for NFS version 4: utf8str_cs, utf8str_cis, and utf8str_mixed. Separate profiles are defined for each. Each profile defines the following, as required by stringprep:

- The intended applicability of the profile 
- The character repertoire that is the input and output to stringprep (which is Unicode 3.2 for referenced version of stringprep)

- The mapping tables from stringprep used (as described in section 3 of stringprep)

- Any additional mapping tables specific to the profile

- The Unicode normalization used, if any (as described in section 4 of stringprep)

- The tables from stringprep listing of characters that are prohibited as output (as described in section 5 of stringprep)

- The bidirectional string testing used, if any (as described in section 6 of stringprep)

- Any additional characters that are prohibited as output specific to the profile

Stringprep discusses Unicode characters, whereas NFS version 4 renders UTF-8 characters. Since there is a one to one mapping from UTF-8 to Unicode, where ever the remainder of this document refers to to Unicode, the reader should assume UTF-8.

Much of the text for the profiles comes from [RFC3454].

11.1. Stringprep profile for the utf8str_cs type

Every use of the utf8str_cs type definition in the NFS version 4 protocol specification follows the profile named nfs4_cs_prep.

11.1.1. Intended applicability of the nfs4_cs_prep profile

The utf8str_cs type is a case sensitive string of UTF-8 characters. Its primary use in NFS Version 4 is for naming components and pathnames. Components and pathnames are stored on the server's filesystem. Two valid distinct UTF-8 strings might be the same after processing via the utf8str_cs profile. If the strings are two names inside a directory, the NFS version 4 server will need to either:

- disallow the creation of a second name if it's post processed form collides with that of an existing name, or

- allow the creation of the second name, but arrange so that after post processing, the second name is different than the post processed form of the first name. 
11.1.2. Character repertoire of nfs4_cs_prep

The nfs4_cs_prep profile uses Unicode 3.2, as defined in stringprep's Appendix A.1

11.1.3. Mapping used by nfs4_cs_prep

The nfs4_cs_prep profile specifies mapping using the following tables from stringprep:

Table B.1

Table B.2 is normally not part of the nfs4_cs_prep profile as it is primarily for dealing with case-insensitive comparisons. However, if the NFS version 4 file server supports the case_insensitive filesystem attribute, and if case_insensitive is true, the NFS version 4 server MUST use Table B.2 (in addition to Table B1) when processing utf8str_cs strings, and the NFS version 4 client MUST assume Table B.2 (in addition to Table B.1) are being used.

If the case_preserving attribute is present and set to false, then the NFS version 4 server MUST use table B.2 to map case when processing utf8str_cs strings. Whether the server maps from lower to upper case or the upper to lower case is an implementation dependency .

11.1.4. Normalization used by nfs4_cs_prep

The nfs4_cs_prep profile does not specify a normalization form. A later revision of this specification may specify a particular normalization form. Therefore, the server and client can expect that they may receive unnormalized characters within protocol requests and responses. If the operating environment requires normalization, then the implementation must normalize utf8str_cs strings within the protocol before presenting the information to an application (at the client) or local filesystem (at the server). 
11.1.5. Prohibited output for nfs4_cs_prep

The nfs4_cs_prep profile specifies prohibiting using the following tables from stringprep:
Table C. 3
Table C. 4
Table C.5
Table C. 6
Table C.7
Table C.8
Table C.9

11.1.6. Bidirectional output for nfs4_cs_prep

The nfs4_cs_prep profile does not specify any checking of bidirectional strings.

11.2. Stringprep profile for the utf8str_cis type

Every use of the utf8str_cis type definition in the NFS version 4 protocol specification follows the profile named nfs4_cis_prep.

11.2.1. Intended applicability of the nfs4_cis_prep profile

The utf8str_cis type is a case insensitive string of UTF-8 characters. Its primary use in NFS Version 4 is for naming NFS servers.

11.2.2. Character repertoire of nfs4_cis_prep

The nfs4_cis_prep profile uses Unicode 3.2, as defined in stringprep's Appendix A.1

11.2.3. Mapping used by nfs4_cis_prep

The nfs4_cis_prep profile specifies mapping using the following tables from stringprep:

Table B.1

Table B.2

11.2.4. Normalization used by nfs4_cis_prep

The nfs4_cis_prep profile specifies using Unicode normalization form $\mathrm{KC}$, as described in stringprep. 
11.2.5. Prohibited output for nfs4_cis_prep

The nfs4_cis_prep profile specifies prohibiting using the following tables from stringprep:
Table C.1.2
Table C.2.2
Table C.3
Table C. 4
Table C.5
Table C. 6
Table C.7
Table C.8
Table C.9

11.2.6. Bidirectional output for nfs4_cis_prep

The nfs4_cis_prep profile specifies checking bidirectional strings as described in stringprep's section 6 .

11.3. Stringprep profile for the utf8str_mixed type

Every use of the utf8str_mixed type definition in the NFS version 4 protocol specification follows the profile named nfs4_mixed_prep.

11.3.1. Intended applicability of the nfs4_mixed_prep profile

The utf8str_mixed type is a string of UTF-8 characters, with a prefix that is case sensitive, a separator equal to 'd', and a suffix that is fully qualified domain name. Its primary use in NFS Version 4 is for naming principals identified in an Access Control Entry.

11.3.2. Character repertoire of nfs4_mixed_prep

The nfs4_mixed_prep profile uses Unicode 3.2, as defined in stringprep's Appendix A.1

11.3.3. Mapping used by nfs4_cis_prep

For the prefix and the separator of a utf8str_mixed string, the nfs4_mixed_prep profile specifies mapping using the following table from stringprep:

Table B.1

For the suffix of a utf8str_mixed string, the nfs4_mixed_prep profile specifies mapping using the following tables from stringprep: 
Table B.1

Table B.2

11.3.4. Normalization used by nfs4_mixed_prep

The nfs4_mixed_prep profile specifies using Unicode normalization form KC, as described in stringprep.

11.3.5. Prohibited output for nfs4_mixed_prep

The nfs4_mixed_prep profile specifies prohibiting using the following tables from stringprep:
Table C.1.2
Table C.2.2
Table C.3
Table C. 4
Table C.5
Table C. 6
Table C.7
Table C.8
Table C.9

11.3.6. Bidirectional output for nfs4_mixed_prep

The nfs4_mixed_prep profile specifies checking bidirectional strings as described in stringprep's section 6 .

11.4. UTF-8 Related Errors

Where the client sends an invalid UTF-8 string, the server should
return an NFS4ER_INVAL error. This includes cases in which
inappropriate prefixes are detected and where the count includes
trailing bytes that do not constitute a full UCS character.
Where the client supplied string is valid UTF-8 but contains
characters that are not supported by the server as a value for that
string (e.g., names containing characters that have more than two
octets on a filesystem that supports Unicode characters only), the
server should return an NFS4ERR_BADCHAR error.
Where a UTF-8 string is used as a file name, and the filesystem,
while supporting all of the characters within the name, does not
allow that particular name to be used, the server should return the
error NFS4ERRBADNAME. This includes situations in which the server
filesystem imposes a normalization constraint on name strings, but 
will also include such situations as filesystem prohibitions of "." and ".." as file names for certain operations, and other such constraints.

\section{Error Definitions}

NFS error numbers are assigned to failed operations within a compound request. A compound request contains a number of NFS operations that have their results encoded in sequence in a compound reply. The results of successful operations will consist of an NFS4_OK status followed by the encoded results of the operation. If an NFS operation fails, an error status will be entered in the reply and the compound request will be terminated.

A description of each defined error follows:

NFS4_OK Indicates the operation completed successfully.

NFS4ERR_ACCESS Permission denied. The caller does not have the correct permission to perform the requested operation. Contrast this with NFS4ERR_PERM, which restricts itself to owner or privileged user permission failures.

NFS4ERR_ATTRNOTSUPP An attribute specified is not supported by the server. Does not apply to the GETATTR operation.

NFS4ERR_ADMIN_REVOKED Due to administrator intervention, the lockowner's record locks, share reservations, and delegations have been revoked by the server.

NFS 4ERR_BADCHAR

A UTF-8 string contains a character which is not supported by the server in the context in which it being used.

NFS 4ERR_BAD_COOKIE

NFS 4ERR_BADHANDLE

NFS 4ERR_BADNAME

Shepler, et al.
READDIR cookie is stale.

Illegal NFS filehandle. The filehandle failed internal consistency checks.

A name string in a request consists of valid UTF-8 characters supported by the server but the name is not supported by the server as a valid name for current operation. 


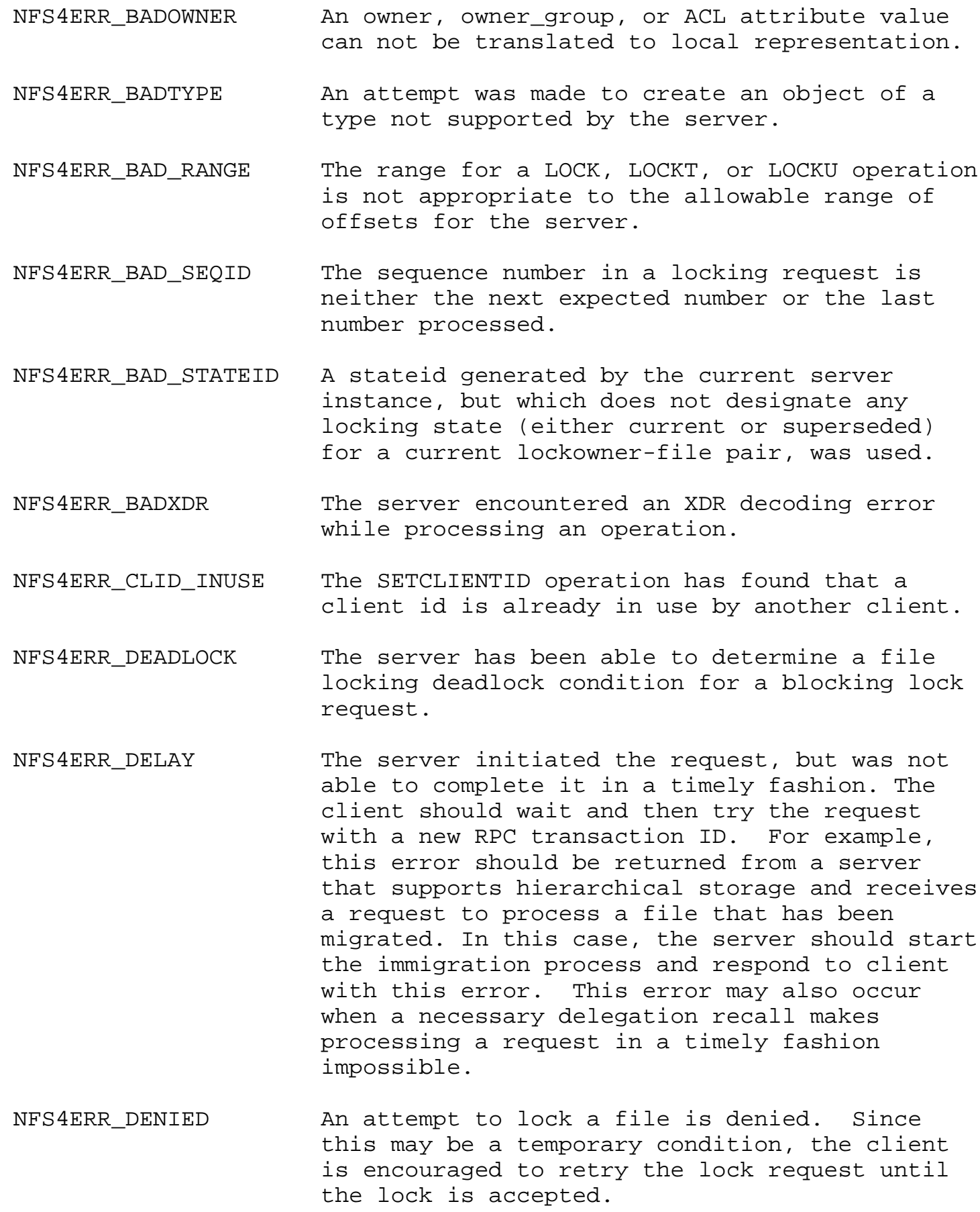
can not be translated to local representation.

An attempt was made to create an object of a type not supported by the server.

The range for a LOCK, LOCKT, or LOCKU operation is not appropriate to the allowable range of offsets for the server.

The sequence number in a locking request is neither the next expected number or the last number processed.

A stateid generated by the current server instance, but which does not designate any locking state (either current or superseded) for a current lockowner-file pair, was used.

The server encountered an XDR decoding error while processing an operation.

The SETCLIENTID operation has found that a client id is already in use by another client.

The server has been able to determine a file locking deadlock condition for a blocking lock request.

The server initiated the request, but was not able to complete it in a timely fashion. The client should wait and then try the request with a new RPC transaction ID. For example, this error should be returned from a server that supports hierarchical storage and receives a request to process a file that has been migrated. In this case, the server should start the immigration process and respond to client with this error. This error may also occur when a necessary delegation recall makes processing a request in a timely fashion impossible.

An attempt to lock a file is denied. Since this may be a temporary condition, the client is encouraged to retry the lock request until the lock is accepted. 


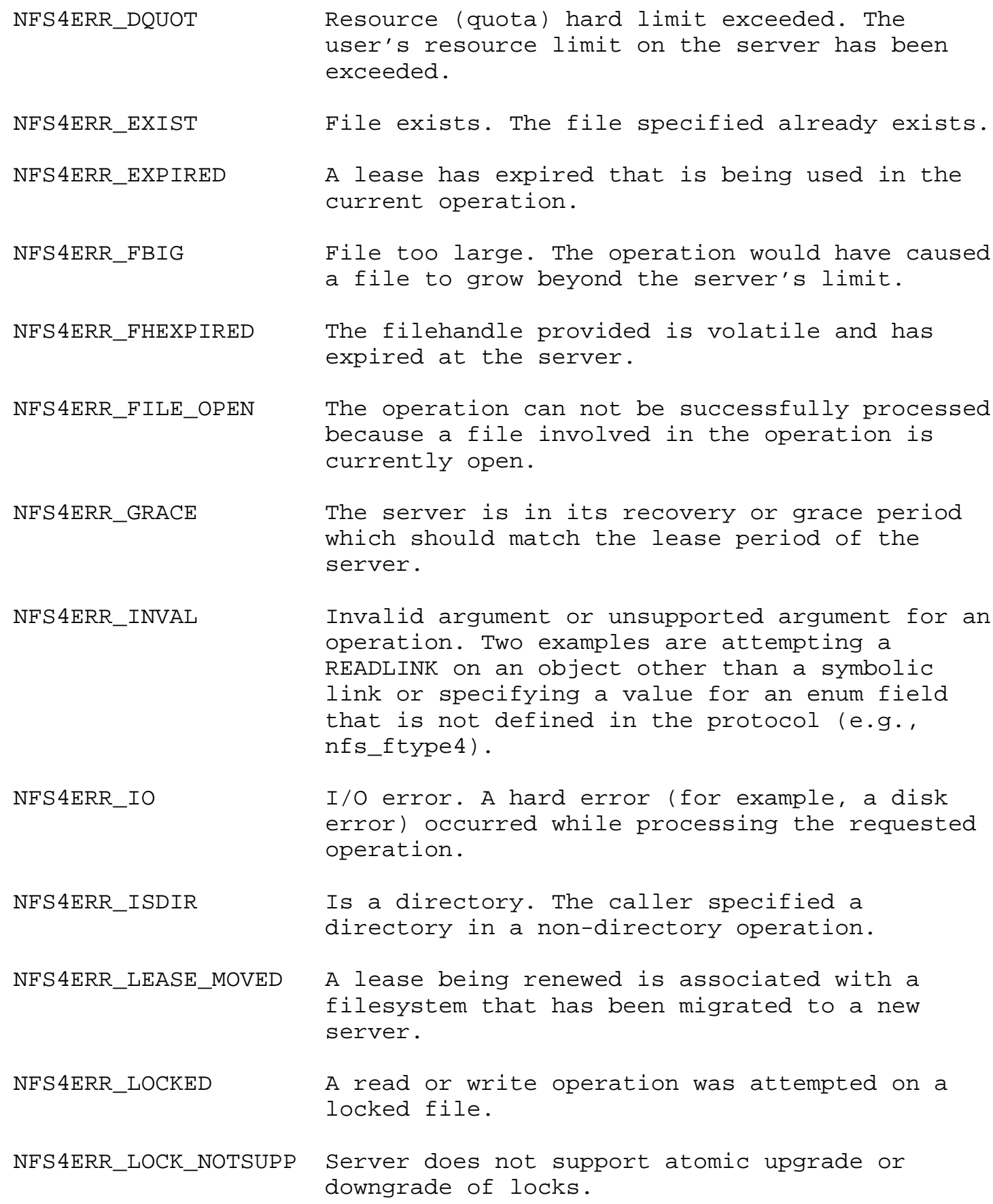
downgrade of locks.

Resource (quota) hard limit exceeded. The user's resource limit on the server has been exceeded.

File exists. The file specified already exists.

A lease has expired that is being used in the current operation.

File too large. The operation would have caused a file to grow beyond the server's limit.

The filehandle provided is volatile and has expired at the server.

The operation can not be successfully processed because a file involved in the operation is currently open.

The server is in its recovery or grace period which should match the lease period of the server.

Invalid argument or unsupported argument for an operation. Two examples are attempting a READLINK on an object other than a symbolic link or specifying a value for an enum field that is not defined in the protocol (e.g., nfs_ftype4).

I/O error. A hard error (for example, a disk error) occurred while processing the requested operation.

Is a directory. The caller specified a directory in a non-directory operation.

A lease being renewed is associated with a filesystem that has been migrated to a new server.

A read or write operation was attempted on a locked file.

Server does not support atomic upgrade or

Standards Track 


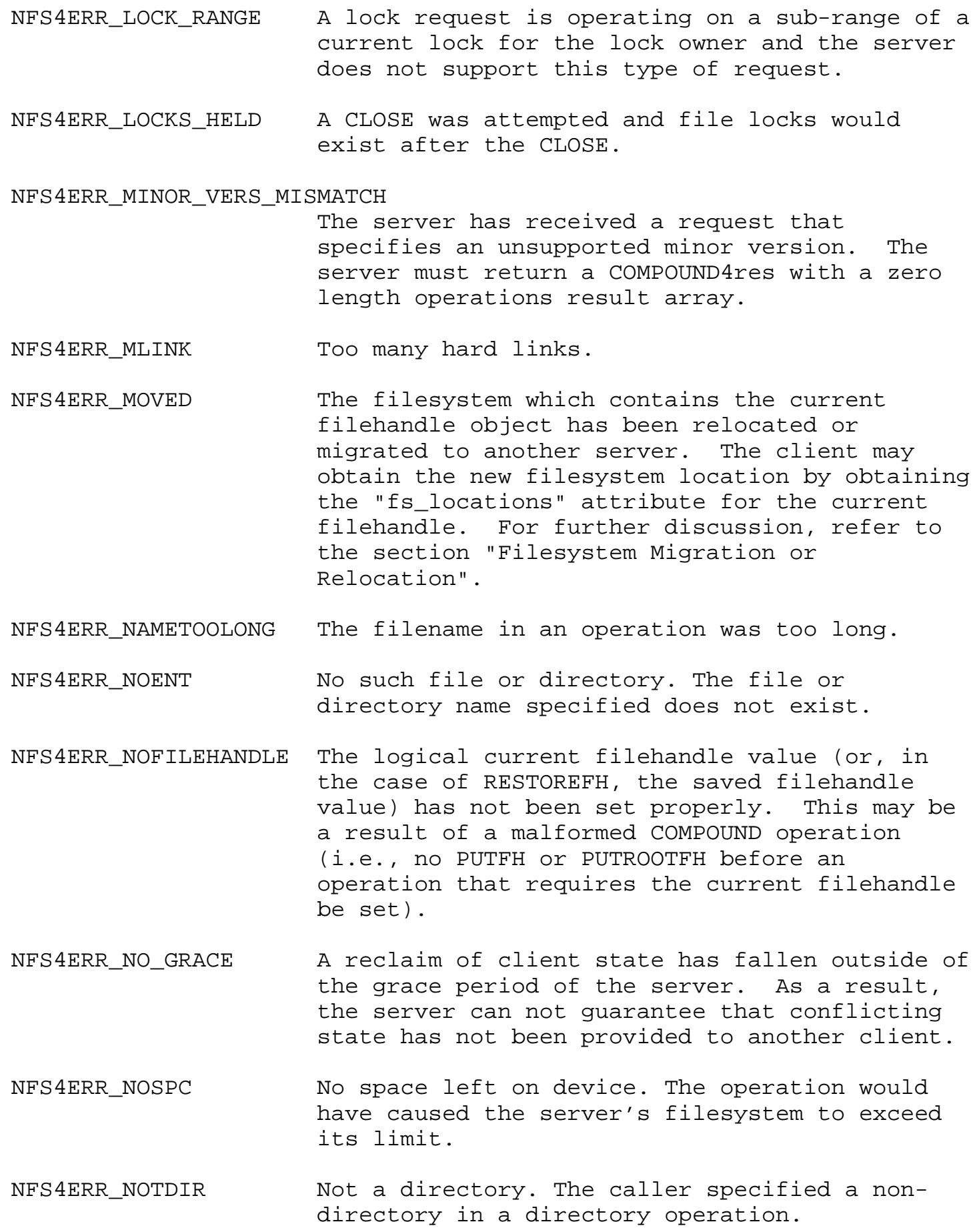

NFS 4ERR_NO_GRACE

NFS 4ERR_NOSPC

NFS 4ERR_NOTDIR

A reclaim of client state has fallen outside of the grace period of the server. As a result, the server can not guarantee that conflicting state has not been provided to another client.

No space left on device. The operation would have caused the server's filesystem to exceed its limit.

Not a directory. The caller specified a nondirectory in a directory operation. 
NFS 4ERR_NOTEMPTY

NFS 4ERR_NOTSUPP

NFS 4ERR_NOT_SAME

NFS 4ERR_NXIO

NFS4ERR_OLD_STATEID

NFS 4ERR_OPENMODE

NFS 4ERR_OP_ILLEGAL

NFS 4ERR_PERM

NF S 4ERR_RECLAIM_BAD

NF S 4ERR_RESOURCE
An attempt was made to remove a directory that was not empty.

Operation is not supported.

This error is returned by the VERIFY operation to signify that the attributes compared were not the same as provided in the client's request.

I/O error. No such device or address.

A stateid which designates the locking state for a lockowner-file at an earlier time was used.

The client attempted a READ, WRITE, LOCK or SETATTR operation not sanctioned by the stateid passed (e.g., writing to a file opened only for read).

An illegal operation value has been specified in the argop field of a COMPOUND or CB_COMPOUND procedure.

Not owner. The operation was not allowed because the caller is either not a privileged user (root) or not the owner of the target of the operation.

The reclaim provided by the client does not match any of the server's state consistency checks and is bad.

NFS 4ERR_RECLAIM_CONFLICT

The reclaim provided by the client has encountered a conflict and can not be provided. potentially indicates a misbehaving client.

For the processing of the COMPOUND procedure, the server may exhaust available resources and can not continue processing operations within the COMPOUND procedure. This error will be returned from the server in those instances of resource exhaustion related to the processing of the COMPOUND procedure. 
NFS 4ERR_RESTOREFH

NFS 4ERR_ROFS

NFS 4ERR_SAME

NFS 4ERR_SERVERFAULT

NFS 4ERR_SHARE_DENIED

NFS 4ERR_STALE

NFS 4ERR_TOOSMALI

NFS 4ERR_WRONGSEC

NF S 4ERR_SYMLINK
The RESTOREFH operation does not have a saved filehandle (identified by SAVEFH) to operate upon.

Read-only filesystem. A modifying operation was attempted on a read-only filesystem.

This error is returned by the NVERIFY operation to signify that the attributes compared were the same as provided in the client's request.

An error occurred on the server which does not map to any of the legal NFS version 4 protocol error values. The client should translate this into an appropriate error. UNIX clients may choose to translate this to EIO.

An attempt to OPEN a file with a share reservation has failed because of a share conflict.

Invalid filehandle. The filehandle given in the arguments was invalid. The file referred to by that filehandle no longer exists or access to it has been revoked.

NFS4ERR_STALE_CLIENTID A clientid not recognized by the server was used in a locking or SETCLIENTID_CONFIRM request.

NFS4ERR_STALE_STATEID A stateid generated by an earlier server instance was used.

The current filehandle provided for a LOOKUP is not a directory but a symbolic link. Also used if the final component of the OPEN path is a symbolic link.

The encoded response to a READDIR request exceeds the size limit set by the initial request.

The security mechanism being used by the client for the operation does not match the server's security policy. The client should change the security mechanism being used and retry the operation.

Shepler, et al.
Standards Track

[Page 133] 
NFS 4ERR_XDEV

Attempt to do an operation between different fsids.

13. NFS version 4 Requests

For the NFS version 4 RPC program, there are two traditional RPC procedures: NULL and COMPOUND. All other functionality is defined as a set of operations and these operations are defined in normal XDR/RPC syntax and semantics. However, these operations are encapsulated within the COMPOUND procedure. This requires that the client combine one or more of the NFS version 4 operations into a single request.

The NFS4_CALLBACK program is used to provide server to client signaling and is constructed in a similar fashion as the NFS version 4 program. The procedures CB_NULL and CB_COMPOUND are defined in the same way as NULL and COMPOUND are within the NFS program. The CB_COMPOUND request also encapsulates the remaining operations of the NFS4_CALLBACK program. There is no predefined RPC program number for the NFS4_CALLBACK program. It is up to the client to specify a program number in the "transient" program range. The program and port number of the NFS4_CALLBACK program are provided by the client as part of the SETCLIENTID/SETCLIENTID_CONFIRM sequence. The program and port can be changed by another SETCLIENTID/SETCLIENTID_CONFIRM sequence, and it is possible to use the sequence to change them within a client incarnation without removing relevant leased client state.

\subsection{Compound Procedure}

The COMPOUND procedure provides the opportunity for better performance within high latency networks. The client can avoid cumulative latency of multiple RPCs by combining multiple dependent operations into a single COMPOUND procedure. A compound operation may provide for protocol simplification by allowing the client to combine basic procedures into a single request that is customized for the client's environment.

The CB_COMPOUND procedure precisely parallels the features of COMPOUND as described above.

The basic structure of the COMPOUND procedure is:

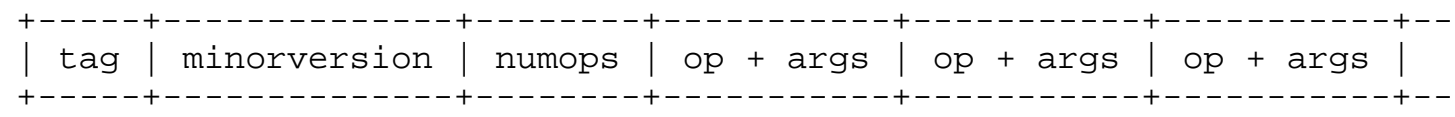


and the reply's structure is:

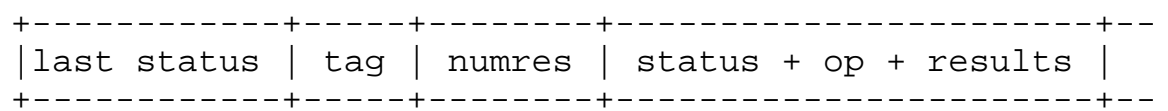

The numops and numres fields, used in the depiction above, represent the count for the counted array encoding use to signify the number of arguments or results encoded in the request and response. As per the XDR encoding, these counts must match exactly the number of operation arguments or results encoded.

\subsection{Evaluation of a Compound Request}

The server will process the COMPOUND procedure by evaluating each of the operations within the COMPOUND procedure in order. Each component operation consists of a 32 bit operation code, followed by the argument of length determined by the type of operation. The results of each operation are encoded in sequence into a reply buffer. The results of each operation are preceded by the opcode and a status code (normally zero). If an operation results in a non-zero status code, the status will be encoded and evaluation of the compound sequence will halt and the reply will be returned. Note that evaluation stops even in the event of "non error" conditions such as NFS4ERR_SAME.

There are no atomicity requirements for the operations contained within the COMPOUND procedure. The operations being evaluated as part of a COMPOUND request may be evaluated simultaneously with other COMPOUND requests that the server receives.

It is the client's responsibility for recovering from any partially completed COMPOUND procedure. Partially completed COMPOUND procedures may occur at any point due to errors such as NFS4ERR_RESOURCE and NFS4ERR_DELAY. This may occur even given an otherwise valid operation string. Further, a server reboot which occurs in the middle of processing a COMPOUND procedure may leave the client with the difficult task of determining how far COMPOUND processing has proceeded. Therefore, the client should avoid overly complex COMPOUND procedures in the event of the failure of an operation within the procedure.

Each operation assumes a "current" and "saved" filehandle that is available as part of the execution context of the compound request. Operations may set, change, or return the current filehandle. The "saved" filehandle is used for temporary storage of a filehandle value and as operands for the RENAME and LINK operations. 


\subsection{Synchronous Modifying Operations}

NFS version 4 operations that modify the filesystem are synchronous. When an operation is successfully completed at the server, the client can depend that any data associated with the request is now on stable storage (the one exception is in the case of the file data in a WRITE operation with the UNSTABLE option specified).

This implies that any previous operations within the same compound request are also reflected in stable storage. This behavior enables the client's ability to recover from a partially executed compound request which may resulted from the failure of the server. For example, if a compound request contains operations A and B and the server is unable to send a response to the client, depending on the progress the server made in servicing the request the result of both operations may be reflected in stable storage or just operation A may be reflected. The server must not have just the results of operation $B$ in stable storage.

13.4. Operation Values

The operations encoded in the COMPOUND procedure are identified by operation values. To avoid overlap with the RPC procedure numbers, operations 0 (zero) and 1 are not defined. Operation 2 is not defined but reserved for future use with minor versioning.

14. NFS version 4 Procedures

14.1. Procedure 0: NULL - No Operation

SYNOPSIS

$<$ null>

ARGUMENT

void;

RESULT

void; 


\section{DESCRIPTION}

Standard NULL procedure. Void argument, void response. This procedure has no functionality associated with it. Because of this it is sometimes used to measure the overhead of processing a service request. Therefore, the server should ensure that no unnecessary work is done in servicing this procedure.

\section{ERRORS}

None.

14.2. Procedure 1: COMPOUND - Compound Operations SYNOPSIS

compoundargs $\rightarrow$ compoundres

ARGUMENT

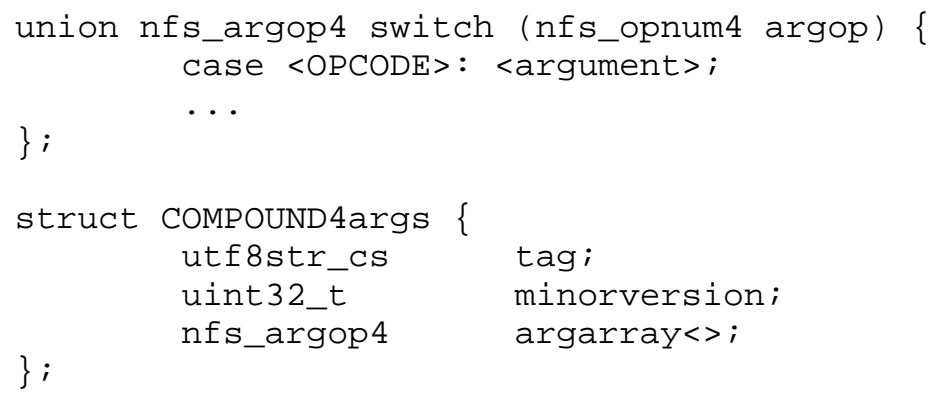




\section{DESCRIPTION}

The COMPOUND procedure is used to combine one or more of the NFS operations into a single RPC request. The main NFS RPC program has two main procedures: NULL and COMPOUND. All other operations use the COMPOUND procedure as a wrapper.

The COMPOUND procedure is used to combine individual operations into a single RPC request. The server interprets each of the operations in turn. If an operation is executed by the server and the status of that operation is NFS4_OK, then the next operation in the COMPOUND procedure is executed. The server continues this process until there are no more operations to be executed or one of the operations has a status value other than NFS4_OK.

In the processing of the COMPOUND procedure, the server may find that it does not have the available resources to execute any or all of the operations within the COMPOUND sequence. In this case, the error NFS4ERR_RESOURCE will be returned for the particular operation within the COMPOUND procedure where the resource exhaustion occurred. This assumes that all previous operations within the COMPOUND sequence have been evaluated successfully. The results for all of the evaluated operations must be returned to the client.

The server will generally choose between two methods of decoding the client's request. The first would be the traditional one-pass XDR decode, in which decoding of the entire COMPOUND precedes execution of any operation within it. If there is an XDR decoding error in this case, an RPC XDR decode error would be returned. The second method would be to make an initial pass to decode the basic COMPOUND request and then to XDR decode each of the individual operations, as the server is ready to execute it. In this case, the server may encounter an XDR decode error during such an operation decode, after previous operations within the COMPOUND have been executed. In this case, the server would return the error NFS4ERR_BADXDR to signify the decode error.

The COMPOUND arguments contain a "minorversion" field. The initial and default value for this field is 0 (zero). This field will be used by future minor versions such that the client can communicate to the server what minor version is being requested. If the server receives a COMPOUND procedure with a minorversion field value that it does not support, the server MUST return an error of NES4ERR_MINOR_VERS_MISMATCH and a zero length resultdata array.

Contained within the COMPOUND results is a "status" field. If the results array length is non-zero, this status must be equivalent to the status of the last operation that was executed within the 
COMPOUND procedure. Therefore, if an operation incurred an error then the "status" value will be the same error value as is being returned for the operation that failed.

Note that operations, 0 (zero) and 1 (one) are not defined for the COMPOUND procedure. Operation 2 is not defined but reserved for future definition and use with minor versioning. If the server receives a operation array that contains operation 2 and the minorversion field has a value of 0 (zero), an error of NFS4ERR_OP_ILLEGAL, as described in the next paragraph, is returned to the client. If an operation array contains an operation 2 and the minorversion field is non-zero and the server does not support the minor version, the server returns an error of NFS4ERR_MINOR_VERS_MISMATCH. Therefore, the NFS4ERR_MINOR_VERS_MISMATCH error takes precedence over all other errors.

It is possible that the server receives a request that contains an operation that is less than the first legal operation (OP_ACCESS) or greater than the last legal operation (OP_RELEASE_LOCKOWNER).

In this case, the server's response will encode the opcode OP_ILLEGAL rather than the illegal opcode of the request. The status field in the ILLEGAL return results will set to NFS4ERR_OP_ILLEGAL. The COMPOUND procedure's return results will also be NFS4ERR_OP_ILLEGAL.

The definition of the "tag" in the request is left to the implementor. It may be used to summarize the content of the compound request for the benefit of packet sniffers and engineers debugging implementations. However, the value of "tag" in the response SHOULD be the same value as provided in the request. This applies to the tag field of the CB_COMPOUND procedure as well.

\section{IMP LEMENTATION}

Since an error of any type may occur after only a portion of the operations have been evaluated, the client must be prepared to recover from any failure. If the source of an NFS4ERR_RESOURCE error was a complex or lengthy set of operations, it is likely that if the number of operations were reduced the server would be able to evaluate them successfully. Therefore, the client is responsible for dealing with this type of complexity in recovery.

ERRORS

All errors defined in the protocol 
14.2.1. Operation 3: ACCESS - Check Access Rights

SYNOPSIS

(cfh), accessreq $\rightarrow$ supported, accessrights

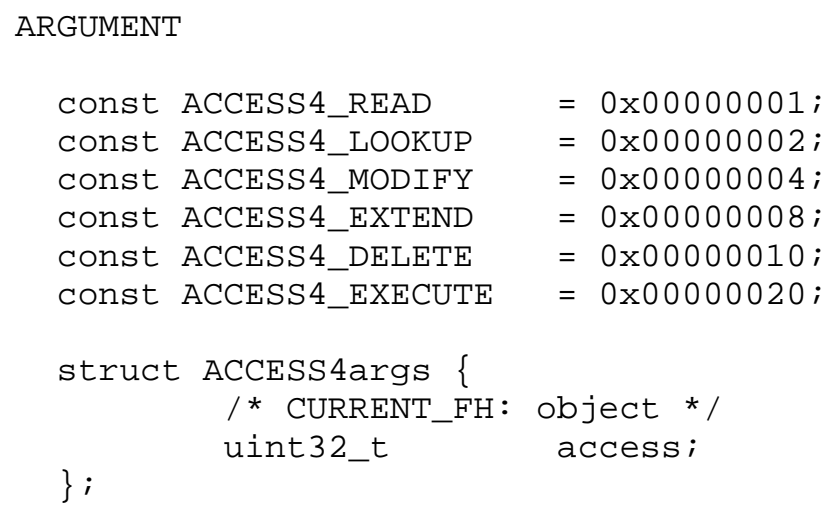


Note that the supported field will contain only as many values as were originally sent in the arguments. For example, if the client sends an ACCESS operation with only the ACCESS4_READ value set and the server supports this value, the server will return only ACCESS4_READ even if it could have reliably checked other values.

The results of this operation are necessarily advisory in nature. A return status of NFS4_OK and the appropriate bit set in the bit mask does not imply that such access will be allowed to the file system object in the future. This is because access rights can be revoked by the server at any time.

The following access permissions may be requested:

ACCESS4_READ Read data from file or read a directory.

ACCESS4_LOOKUP Look up a name in a directory (no meaning for nondirectory objects).

ACCESS4_MODIFY Rewrite existing file data or modify existing directory entries.

ACCESS4_EXTEND Write new data or add directory entries.

ACCESS4_DELETE Delete an existing directory entry.

ACCESS4_EXECUTE Execute file (no meaning for a directory).

On success, the current filehandle retains its value.

IMP LEMENTATION

In general, it is not sufficient for the client to attempt to deduce access permissions by inspecting the uid, gid, and mode fields in the file attributes or by attempting to interpret the contents of the ACL attribute. This is because the server may perform uid or gid mapping or enforce additional access control restrictions. It is also possible that the server may not be in the same ID space as the client. In these cases (and perhaps others), the client can not reliably perform an access check with only current file attributes.

In the NFS version 2 protocol, the only reliable way to determine whether an operation was allowed was to try it and see if it succeeded or failed. Using the ACCESS operation in the NFS version 4 protocol, the client can ask the server to indicate whether or not one or more classes of operations are permitted. The ACCESS operation is provided to allow clients to check before doing a series of operations which will result in an access failure. The OPEN 
operation provides a point where the server can verify access to the file object and method to return that information to the client. The ACCESS operation is still useful for directory operations or for use in the case the UNIX API "access" is used on the client.

The information returned by the server in response to an ACCESS call is not permanent. It was correct at the exact time that the server performed the checks, but not necessarily afterwards. The server can revoke access permission at any time.

The client should use the effective credentials of the user to build the authentication information in the ACCESS request used to determine access rights. It is the effective user and group credentials that are used in subsequent read and write operations.

Many implementations do not directly support the ACCESS4_DELETE permission. Operating systems like UNIX will ignore the ACCESS4_DELETE bit if set on an access request on a non-directory object. In these systems, delete permission on a file is determined by the access permissions on the directory in which the file resides, instead of being determined by the permissions of the file itself. Therefore, the mask returned enumerating which access rights can be determined will have the ACCESS4_DELETE value set to 0 . This indicates to the client that the server was unable to check that particular access right. The ACCESS4_DELETE bit in the access mask returned will then be ignored by the client.

\section{ERRORS}

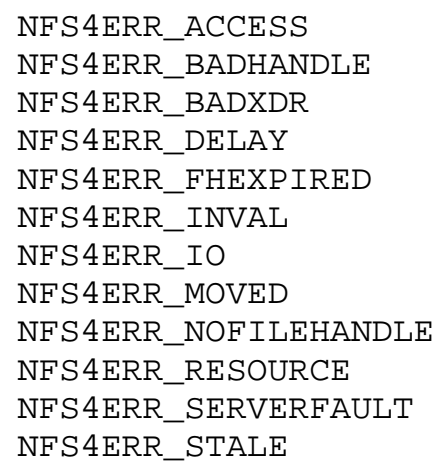




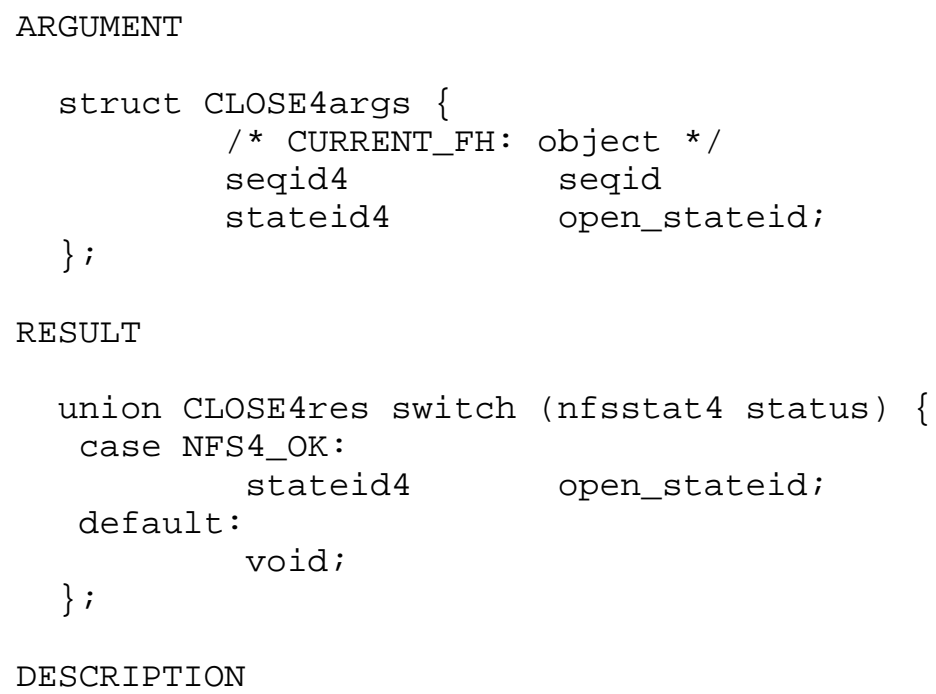




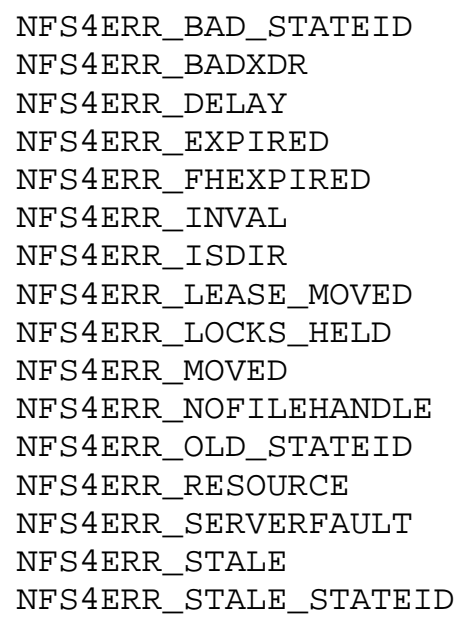

14.2.3. Operation 5: COMMIT - Commit Cached Data SYNOPSIS

(cfh), offset, count $\rightarrow$ verifier

ARGUMENT

struct COMMIT4args \{ /* CURRENT_FH: file */ offset 4 offset;

\} ; count 4 count;

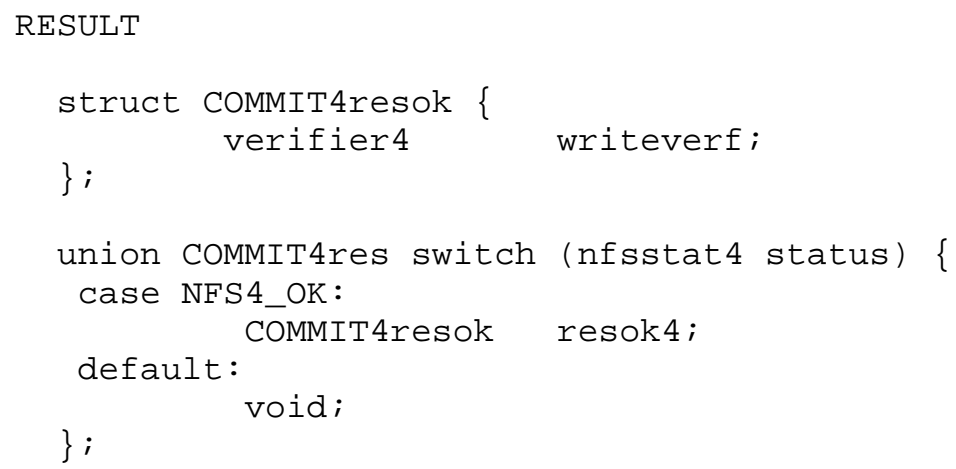




\section{DESCRIPTION}

The COMMIT operation forces or flushes data to stable storage for the file specified by the current filehandle. The flushed data is that which was previously written with a WRITE operation which had the stable field set to UNSTABLE4.

The offset specifies the position within the file where the flush is to begin. An offset value of 0 (zero) means to flush data starting at the beginning of the file. The count specifies the number of bytes of data to flush. If count is 0 (zero), a flush from offset to the end of the file is done.

The server returns a write verifier upon successful completion of the COMMIT. The write verifier is used by the client to determine if the server has restarted or rebooted between the initial WRITE(s) and the COMMIT. The client does this by comparing the write verifier returned from the initial writes and the verifier returned by the COMMIT operation. The server must vary the value of the write verifier at each server event or instantiation that may lead to a loss of uncommitted data. Most commonly this occurs when the server is rebooted; however, other events at the server may result in uncommitted data loss as well.

On success, the current filehandle retains its value.

\section{IMP LEMENTATION}

The COMMIT operation is similar in operation and semantics to the POSIX fsync(2) system call that synchronizes a file's state with the disk (file data and metadata is flushed to disk or stable storage). COMMIT performs the same operation for a client, flushing any unsynchronized data and metadata on the server to the server's disk or stable storage for the specified file. Like fsync(2), it may be that there is some modified data or no modified data to synchronize. The data may have been synchronized by the server's normal periodic buffer synchronization activity. COMMIT should return NFS4_OK, unless there has been an unexpected error.

COMMIT differs from fsync(2) in that it is possible for the client to flush a range of the file (most likely triggered by a bufferreclamation scheme on the client before file has been completely written).

The server implementation of COMMIT is reasonably simple. If the server receives a full file COMMIT request, that is starting at offset 0 and count 0 , it should do the equivalent of fsync()'ing the file. Otherwise, it should arrange to have the cached data in the 
range specified by offset and count to be flushed to stable storage. In both cases, any metadata associated with the file must be flushed to stable storage before returning. It is not an error for there to be nothing to flush on the server. This means that the data and metadata that needed to be flushed have already been flushed or lost during the last server failure.

The client implementation of COMMIT is a little more complex. There are two reasons for wanting to commit a client buffer to stable storage. The first is that the client wants to reuse a buffer. In this case, the offset and count of the buffer are sent to the server in the COMMIT request. The server then flushes any cached data based on the offset and count, and flushes any metadata associated with the file. It then returns the status of the flush and the write verifier. The other reason for the client to generate a COMMIT is for a full file flush, such as may be done at close. In this case, the client would gather all of the buffers for this file that contain uncommitted data, do the COMMIT operation with an offset of 0 and count of 0 , and then free all of those buffers. Any other dirty buffers would be sent to the server in the normal fashion.

After a buffer is written by the client with the stable parameter set to UNSTABLE4, the buffer must be considered as modified by the client until the buffer has either been flushed via a COMMIT operation or written via a WRITE operation with stable parameter set to FILE_SYNC4 or DATA_SYNC4. This is done to prevent the buffer from being freed and reused before the data can be flushed to stable storage on the server.

When a response is returned from either a WRITE or a COMMIT operation and it contains a write verifier that is different than previously returned by the server, the client will need to retransmit all of the buffers containing uncommitted cached data to the server. How this is to be done is up to the implementor. If there is only one buffer of interest, then it should probably be sent back over in a WRITE request with the appropriate stable parameter. If there is more than one buffer, it might be worthwhile retransmitting all of the buffers in WRITE requests with the stable parameter set to UNSTABLE 4 and then retransmitting the COMMIT operation to flush all of the data on the server to stable storage. The timing of these retransmissions is left to the implementor.

The above description applies to page-cache-based systems as well as buffer-cache-based systems. In those systems, the virtual memory system will need to be modified instead of the buffer cache. 


\section{ERRORS}

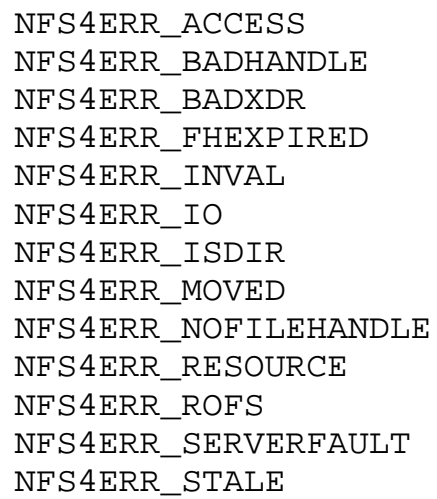

14.2.4. Operation 6: CREATE - Create a Non-Regular File Object SYNOPSIS

(cfh), name, type, attrs $->$ (cfh), change_info, attrs_set ARGUMENT

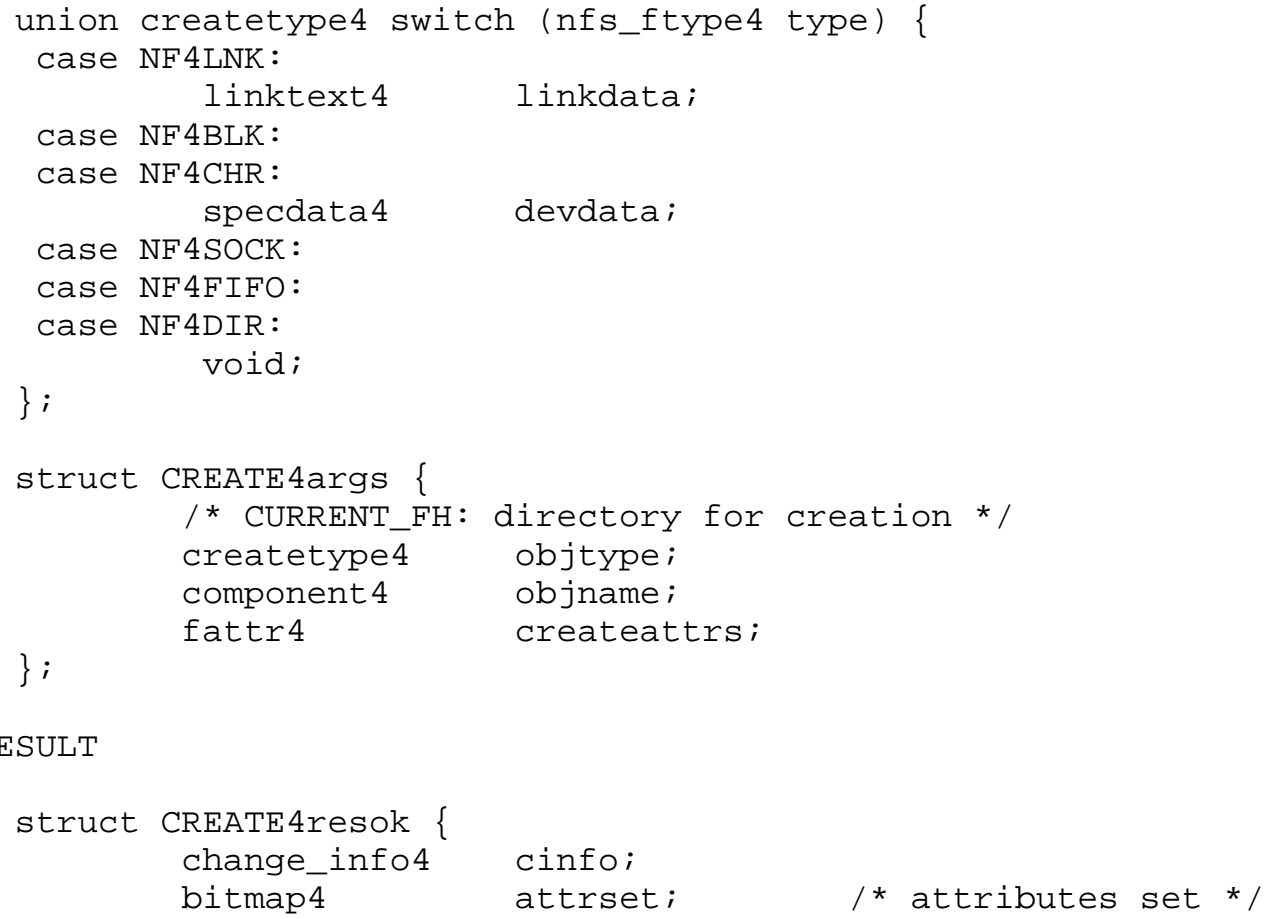




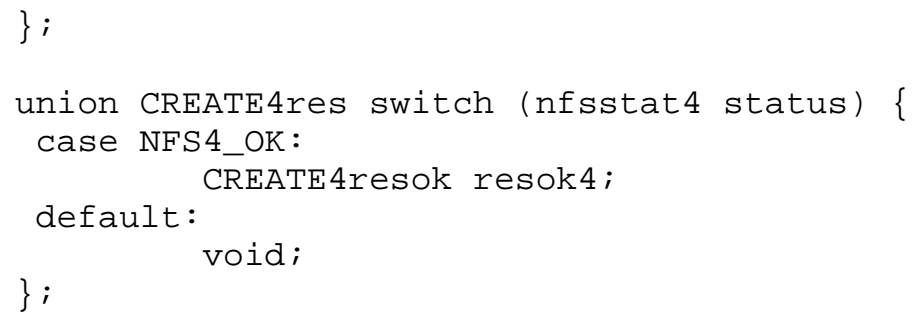




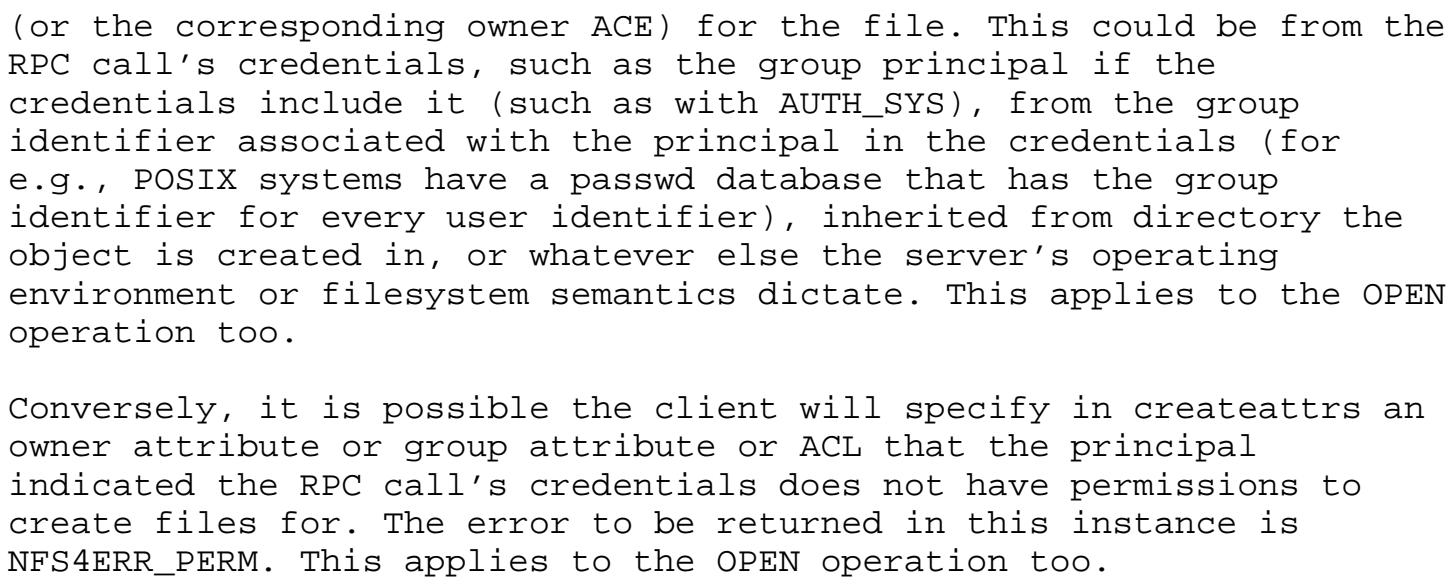

NFS 4ERR_ACCESS
NFS 4ERR_ATTRNOTSUPP
NFS 4ERR_BADCHAR
NFS 4ERR_BADHANDLE
NFS 4ERR_BADNAME
NFS 4ERR_BADOWNER
NFS 4ERR_BADTYPE
NFS 4ERR_BADXDR
NFS 4ERR_DELAY
NFS 4ERR_DQUOT
NFS 4ERR_EXIST
NFS 4ERR_FHEXP IRED
NFS 4ERR_INVAL
NFS 4ERR_IO
NFS 4ERR_MOVED
NFS 4ERR_NAMETOOLONG
NFS 4ERR_NOFILEHANDLE
NFS 4ERR_NOSPC
NFS 4ERR_NOTDIR
NFS 4ERR_PERM
NFS 4ERR_RESOURCE
NFS 4ERR_ROFS
NFS 4ERR_SERVERFAULT
NFS 4ERR_STALE


14.2.5. Operation 7: DELEGPURGE - Purge Delegations Awaiting Recovery

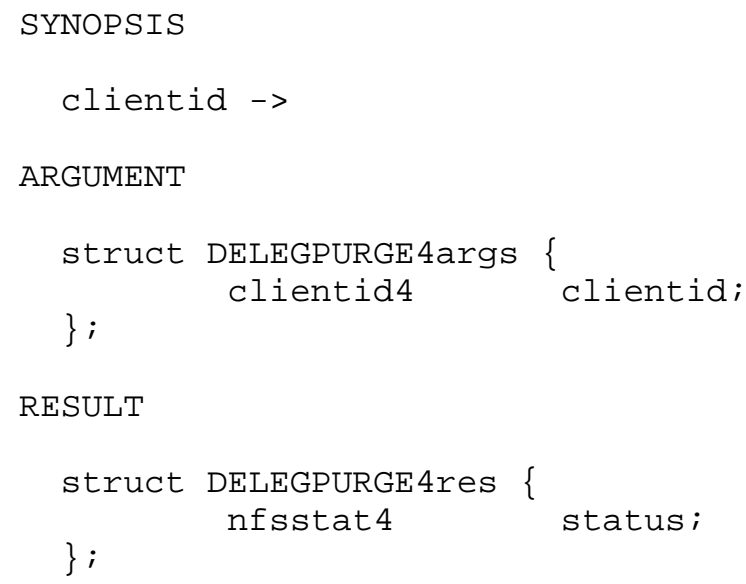


NF S 4ERR_SERVERFAULT

NFS 4ERR_STALE_CLIENTID

14.2.6. Operation 8: DELEGRETURN - Return Delegation

SYNOPSIS

$(\mathrm{cfh})$, stateid $->$

ARGUMENT

struct DELEGRETURN4args \{ / CURRENT_FH: delegated file */

\} ;

$$
\text { stateid4 stateid; }
$$

RESULT

struct DELEGRETURN4res \{

$$
\text { nfsstat } 4 \text { status; }
$$

\};

DESCRIPTION

Returns the delegation represented by the current filehandle and stateid.

Delegations may be returned when recalled or voluntarily (i.e., before the server has recalled them). In either case the client must properly propagate state changed under the context of the delegation to the server before returning the delegation.

\section{ERRORS}

NFS 4ERR_ADMIN_REVOKED
NFS 4 ERR_BAD_STATEID
NFS 4 ERR_BADXDR
NFS 4 ERR_EXPIRED
NFS 4 ERR_INVAL
NFS 4ERR_LEASE_MOVED
NFS 4ERR_MOVED
NFS 4 ERR_NOFILEHANDLE
NFS 4ERR_NOTSUPP
NFS 4ERR_OLD_STATEID
NFS 4ERR_RESOURCE
NFS 4 ERR_SERVERFAULT
NFS 4ERR_STALE
NFS 4ERR_STALE_STATEID


14.2.7. Operation 9: GETATTR - Get Attributes

SYNOPSIS

$(\mathrm{cfh})$, attrbits $\rightarrow$ attrbits, attrvals

ARGUMENT

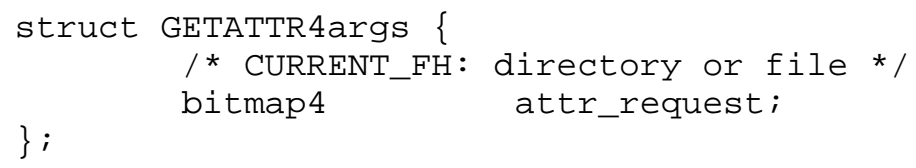




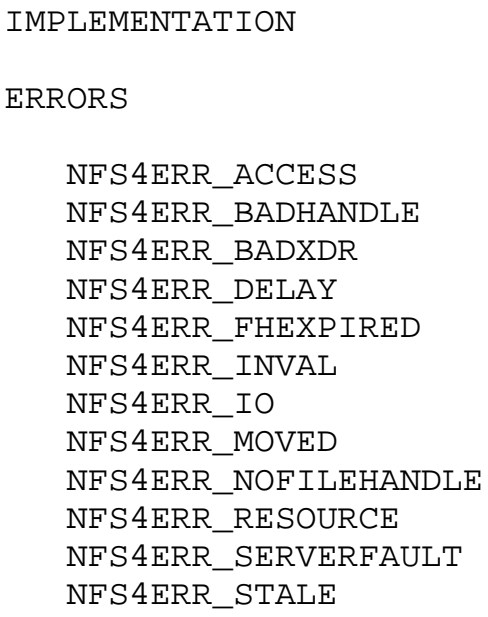




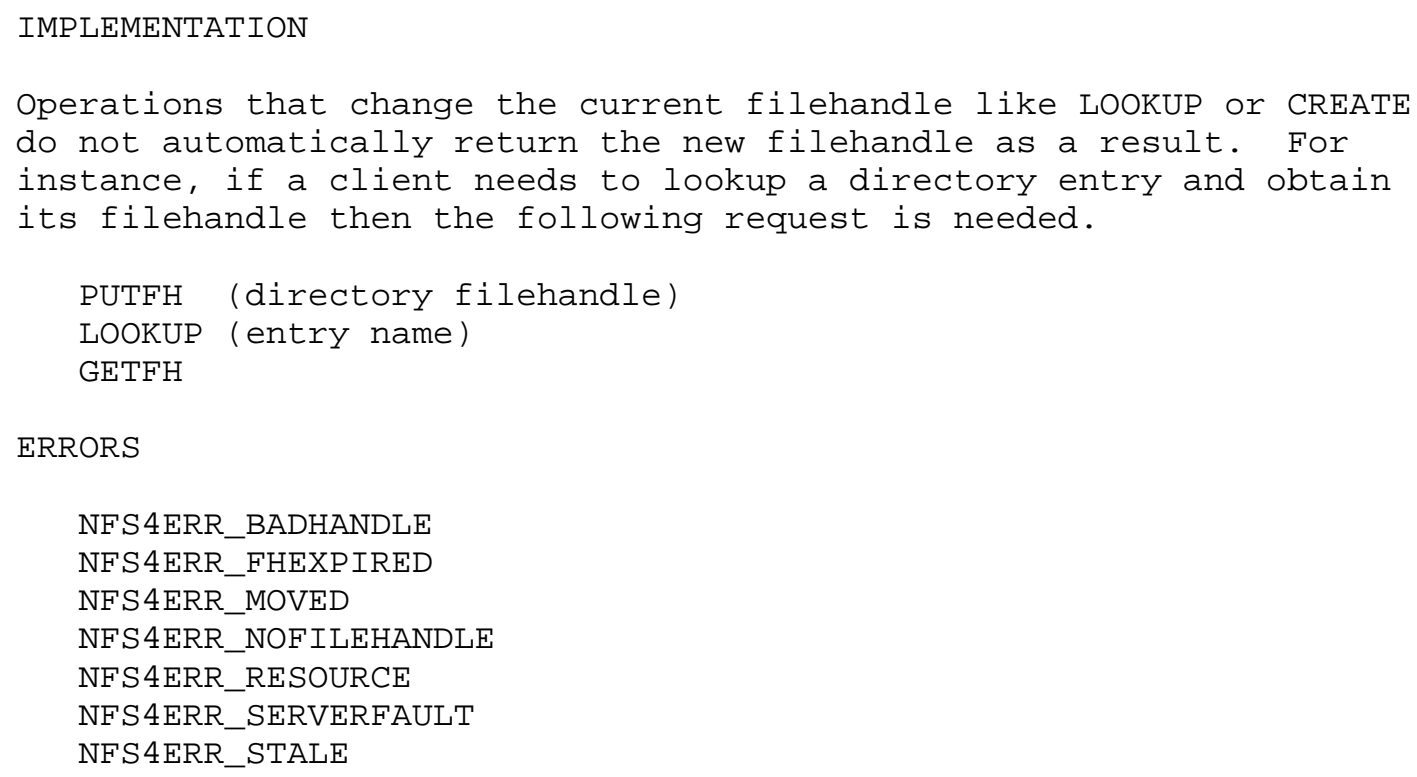




\section{DESCRIPTION}

The LINK operation creates an additional newname for the file represented by the saved filehandle, as set by the SAVEFH operation, in the directory represented by the current filehandle. The existing file and the target directory must reside within the same filesystem on the server. On success, the current filehandle will continue to be the target directory. If an object exists in the target directory with the same name as newname, the server must return NFS4ERR_EXIST.

For the target directory, the server returns change_info4 information in cinfo. With the atomic field of the change_info4 struct, the server will indicate if the before and after change attributes were obtained atomically with respect to the link creation.

If the newname has a length of 0 (zero), or if newname does not obey the UTF-8 definition, the error NFS4ERR_INVAL will be returned.

\section{IMP LEMENTATION}

Changes to any property of the "hard" linked files are reflected in all of the linked files. When a link is made to a file, the attributes for the file should have a value for numlinks that is one greater than the value before the LINK operation.

The statement "file and the target directory must reside within the same filesystem on the server" means that the fsid fields in the attributes for the objects are the same. If they reside on different filesystems, the error, NFS4ERR_XDEV, is returned. On some servers, the filenames, "." and "..", are illegal as newname.

In the case that newname is already linked to the file represented by the saved filehandle, the server will return NFS4ERR_EXIST.

Note that symbolic links are created with the CREATE operation.

\section{ERRORS}

NFS 4ERR_ACCESS
NFS 4 ERR_BADCHAR
NFS 4 ERR_BADHANDLE
NFS 4 ERR_BADNAME
NFS 4 ERR_BADXDR
NFS 4 ERR_DELAY
NFS 4 ERR_DQUOT
NFS 4 ERR_EXIST
NFS 4 ERR_FHEXP IRED
NFS 4ERR_FILE_OPEN




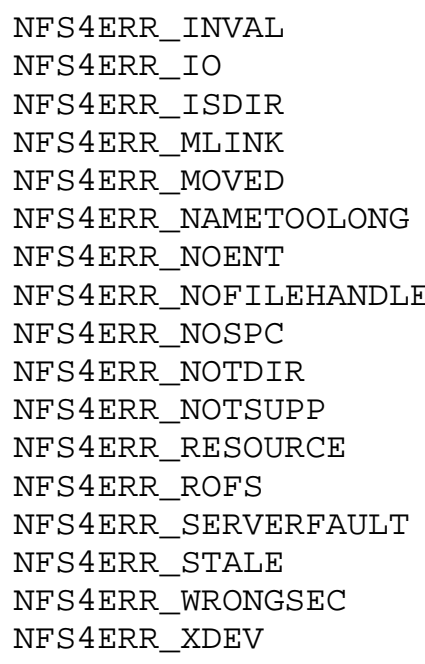

14.2.10. Operation 12: LOCK - Create Lock SYNOPSIS

(cfh) locktype, reclaim, offset, length, locker $->$ stateid ARGUMENT

struct open_to_lock_owner4 \{ seqid4 open_seqid; stateid4 open_stateid; seqid4 lock_seqid;

\} ; lock_owner4 lock_owner;

struct exist lock owner4 stateid4 lock_stateid;

\} ; seqid4 lock_seqid;

union locker4 switch (bool new_lock_owner) \{ case TRUE: open_to_lock_owner4 open_owner; case FALSE:

\} ; exist_lock_owner4 lock_owner;

enum nfs_lock_type4 \{ READ_LT $=1$, WRITE_LT $=2$, 


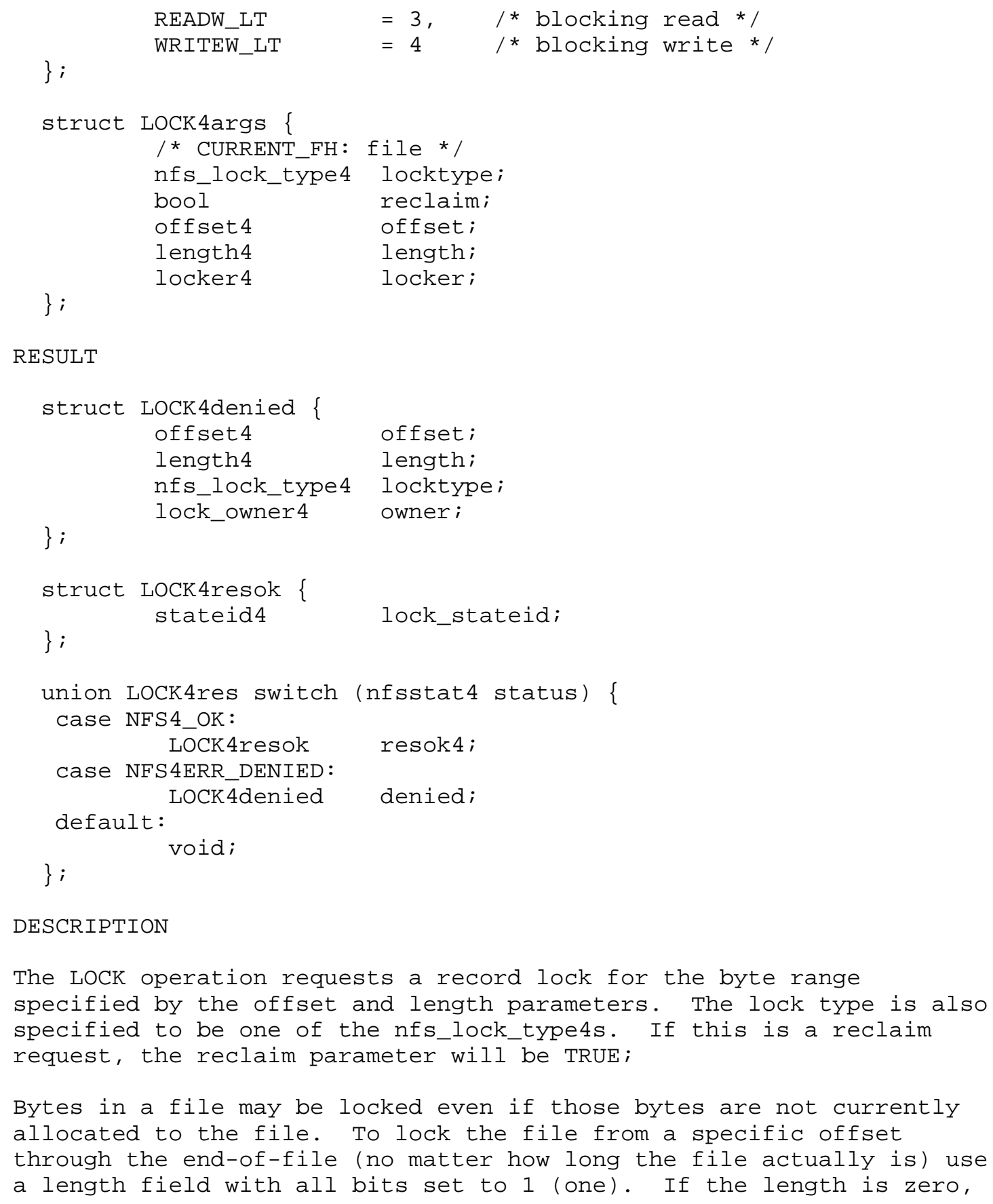


or if a length which is not all bits set to one is specified, and length when added to the offset exceeds the maximum 64-bit unsigned integer value, the error NFS4ERR_INVAL will result.

Some servers may only support locking for byte offsets that fit within 32 bits. If the client specifies a range that includes a byte beyond the last byte offset of the 32-bit range, but does not include the last byte offset of the 32-bit and all of the byte offsets beyond it, up to the end of the valid 64-bit range, such a 32-bit server MUST return the error NFS4ERR_BAD_RANGE.

In the case that the lock is denied, the owner, offset, and length of a conflicting lock are returned.

On success, the current filehandle retains its value.

\section{IMP LEMENTATION}

If the server is unable to determine the exact offset and length of the conflicting lock, the same offset and length that were provided in the arguments should be returned in the denied results. The File Locking section contains a full description of this and the other file locking operations.

LOCK operations are subject to permission checks and to checks against the access type of the associated file. However, the specific right and modes required for various type of locks, reflect the semantics of the server-exported filesystem, and are not specified by the protocol. For example, Windows 2000 allows a write lock of a file open for READ, while a POSIX-compliant system does not.

When the client makes a lock request that corresponds to a range that the lockowner has locked already (with the same or different lock type), or to a sub-region of such a range, or to a region which includes multiple locks already granted to that lockowner, in whole or in part, and the server does not support such locking operations (i.e., does not support POSIX locking semantics), the server will return the error NFS4ERR_LOCK_RANGE. In that case, the client may return an error, or it may emulate the required operations, using only Lock for ranges that do not include any bytes already locked by that lock_owner and LOCKU of locks held by that lock_owner (specifying an exactly-matching range and type). Similarly, when the client makes a lock request that amounts to upgrading (changing from a read lock to a write lock) or downgrading (changing from write lock to a read lock) an existing record lock, and the server does not 
support such a lock, the server will return NFS4ERR_LOCK_NOTSUPP. Such operations may not perfectly reflect the required semantics in the face of conflicting lock requests from other clients.

The locker argument specifies the lock_owner that is associated with the LOCK request. The locker4 structure is a switched union that indicates whether the lock_owner is known to the server or if the lock_owner is new to the server. In the case that the lock_owner is known to the server and has an established lock_seqid, the argument is just the lock_owner and lock_seqid. In the case that the lock_owner is not known to the server, the argument contains not only the lock_owner and lock_seqid but also the open_stateid and open_seqid. The new lock_owner case covers the very first lock done by the lock_owner and offers a method to use the established state of the open_stateid to transition to the use of the lock_owner.

\section{ERRORS}

NFS 4ERR_ACCESS

NFS 4ERR_ADMIN_REVOKED

NFS 4ERR_BADHANDLE

NFS 4ERR_BAD_RANGE

NF S 4ERR_BAD_SEQID

NFS 4ERR_BAD_STATEID

NFS 4ERR_BADXDR

NFS 4ERR_DEADLOCK

NFS 4ERR_DELAY

NFS 4ERR_DENIED

NFS 4ERR_EXPIRED

NFS 4ERR_FHEXP IRED

NFS 4ERR_GRACE

NFS 4ERR_INVAL

NFS4ERR_ISDIR

NFS 4ERR_LEASE_MOVED

NF S 4ERR_LOCK_NOTSUPP

NFS 4ERR_LOCK_RANGE

NFS 4ERR_MOVED

NFS 4ERR_NOF I LEHANDLE

NFS 4ERR_NO_GRACE

NFS 4ERR_OLD_STATEID

NFS 4ERR_OPENMODE

NFS 4ERR_RECLAIM_BAD

NES 4ERR_RECLAIM_CONFLICT

NFS 4ERR_RESOURCE

NFS 4ERR_SERVERFAULT

NFS 4ERR_STALE

NFS 4ERR_STALE_CLIENTID

NFS4ERR_STALE_STATEID 
14.2.11. Operation 13: LOCKT - Test For Lock

SYNOPSIS

(cfh) locktype, offset, length owner $\rightarrow$ \{void, NFS4ERR_DENIED $\rightarrow>$ owner\}

ARGUMENT

struct LOCKT4args \{

/* CURRENT_FH: file */

nfs_lock_type4 locktype;

offset 4 offset;

length4 length;

\} ;

lock_owner4 owner;

RESULT

struct LOCK4denied \{ offset 4 offset;

length4 length;

nfs_lock_type 4 locktype;

\} ;

lock_owner4 owner;

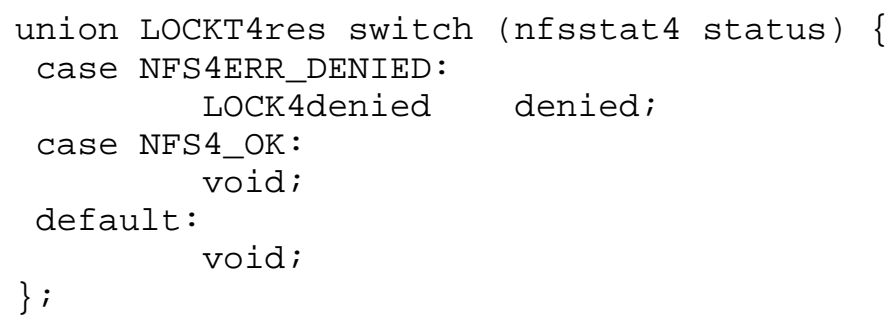


On success, the current filehandle retains its value.

IMP LEMENTATION

If the server is unable to determine the exact offset and length of the conflicting lock, the same offset and length that were provided in the arguments should be returned in the denied results. The File Locking section contains further discussion of the file locking mechanisms.

LOCKT uses a lock_owner4 rather a stateid4, as is used in LOCK to identify the owner. This is because the client does not have to open the file to test for the existence of a lock, so a stateid may not be available.

The test for conflicting locks should exclude locks for the current lockowner. Note that since such locks are not examined the possible existence of overlapping ranges may not affect the results of LOCKT. If the server does examine locks that match the lockowner for the purpose of range checking, NFS4ERR_LOCK_RANGE may be returned.. In the event that it returns NFS4_OK, clients may do a LOCK and receive NFS4ERR_LOCK_RANGE on the LOCK request because of the flexibility provided to the server.

\section{ERRORS}

NFS 4ERR_ACCESS

NF S 4ERR_BADHANDLE

NFS 4ERR_BAD_RANGE

NFS 4ERR_BADXDR

NF S 4ERR_DELAY

NFS 4ERR_DENIED

NF S 4ERR_FHEXP IRED

NFS 4ERR_GRACE

NFS 4ERR_INVAL

NFS 4ERR_ISDIR

NFS 4ERR_LEASE_MOVED

NFS 4ERR_LOCK_RANGE

NFS 4ERR_MOVED

NFS 4ERR_NOF I LEHANDLE

NFS 4ERR_RESOURCE

NFS 4ERR_SERVERFAULT

NFS 4ERR_STALE

NFS 4ERR_STALE_CLIENTID 
14.2.12. Operation 14: LOCKU - Unlock File

SYNOPSIS

(cfh) type, seqid, stateid, offset, length $\rightarrow$ stateid

ARGUMENT

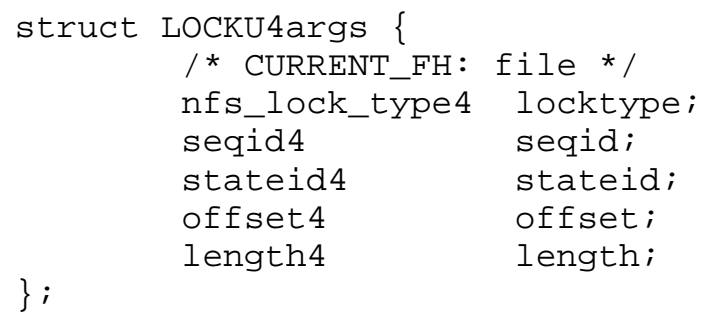




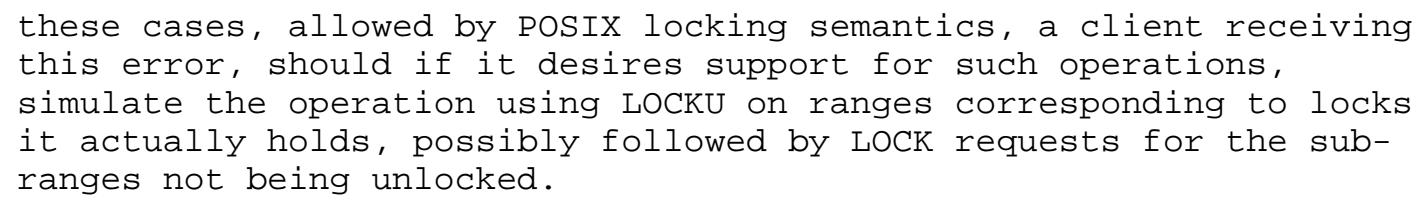

14.2.13. Operation 15: LoOKUP - Lookup Filename SYNOPSIS

$(\mathrm{cfh})$, component $->$ (cfh)

ARGUMENT

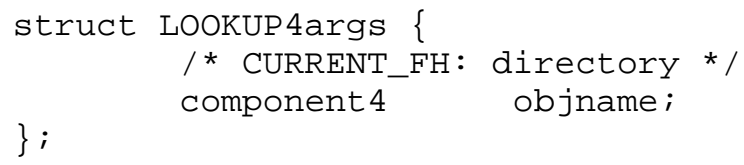


RESULT

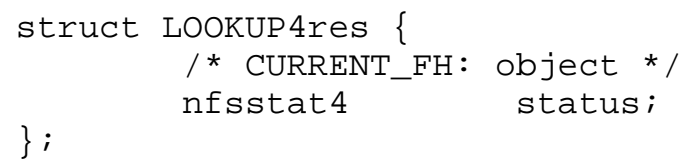




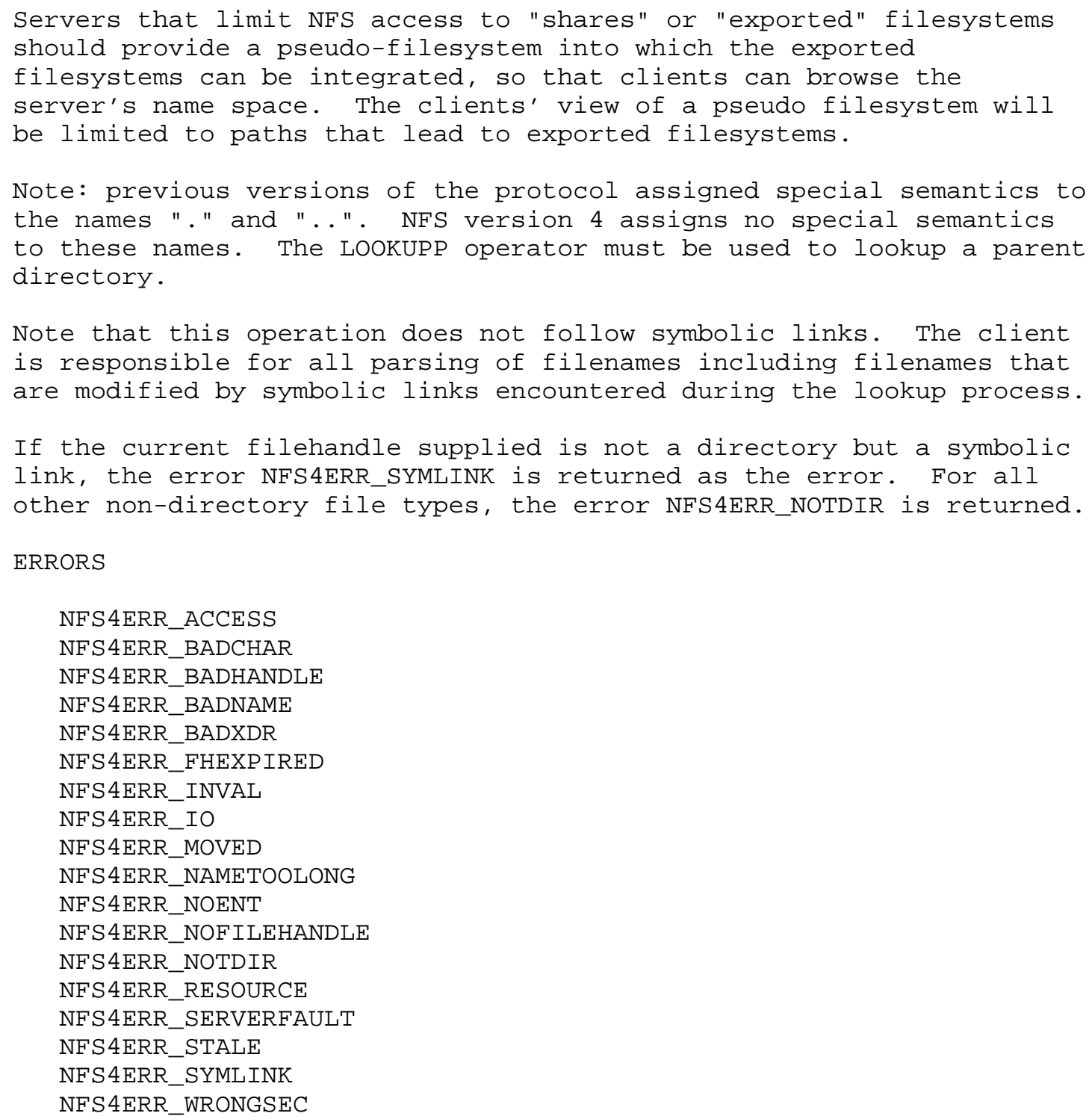




\section{ARGUMENT}

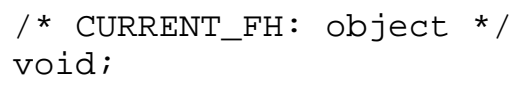

14.2.15. Operation 17: NVERIFY - Verify Difference in Attributes SYNOPSIS

(cfh), fattr $->-$ 


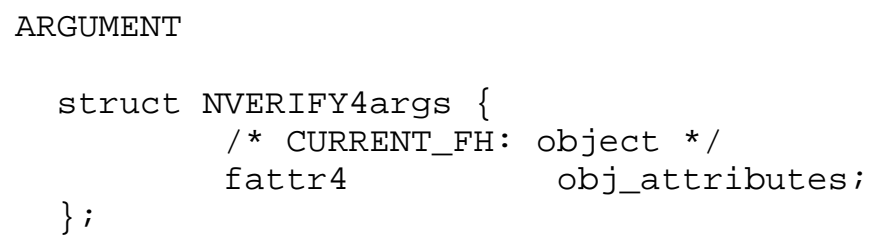

In the case that a recommended attribute is specified in the NVERIFY operation and the server does not support that attribute for the filesystem object, the error NFS4ERR_ATTRNOTSUPP is returned to the client.

When the attribute rdattr_error or any write-only attribute (e.g., time_modify_set) is specified, the error NFS4ERR_INVAL is returned to the client. 


\section{ERRORS}

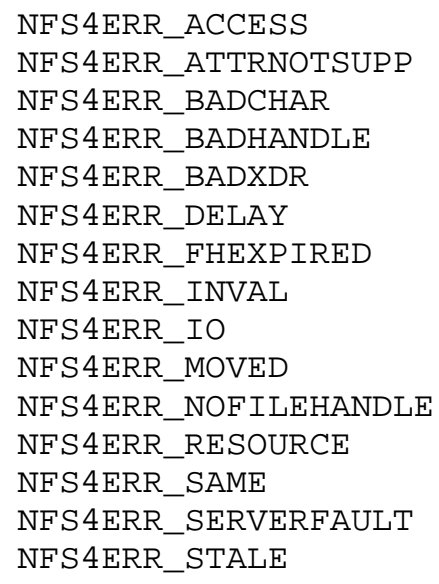

14.2.16. Operation 18: OPEN - Open a Regular File SYNOPSIS

(cfh), seqid, share_access, share_deny, owner, openhow, claim -> (cfh), stateid, cinfo, rflags, open_confirm, attrset delegation

\section{ARGUMENT}

struct OPEN4args \{ seqid4 seqid; uint32_t share_access; uint32_t share_deny; open_owner4 owner; openflag4 openhow;

\} ; open_claim4 claim;

enum createmode 4 UNCHECKED $4=0$, GUARDED $4=1$ EXCLUSIVE $4=2$

\} ;

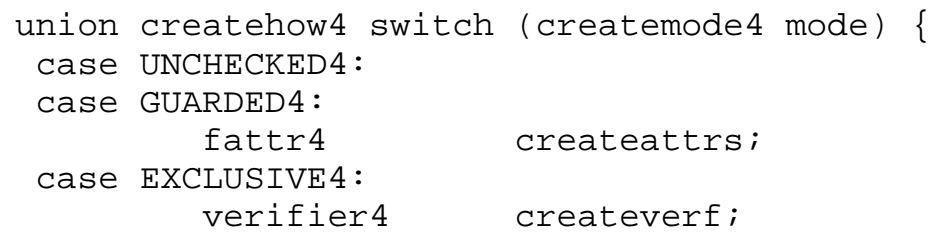


\};

enum opentype 4

$$
\text { OPEN4_NOCREATE }=0 \text {, }
$$

\};

OPEN4_CREATE $=1$

union openflag4 switch (opentype4 opentype) \{ case OPEN4_CREATE:

default: createhow 4 how;

\}; void;

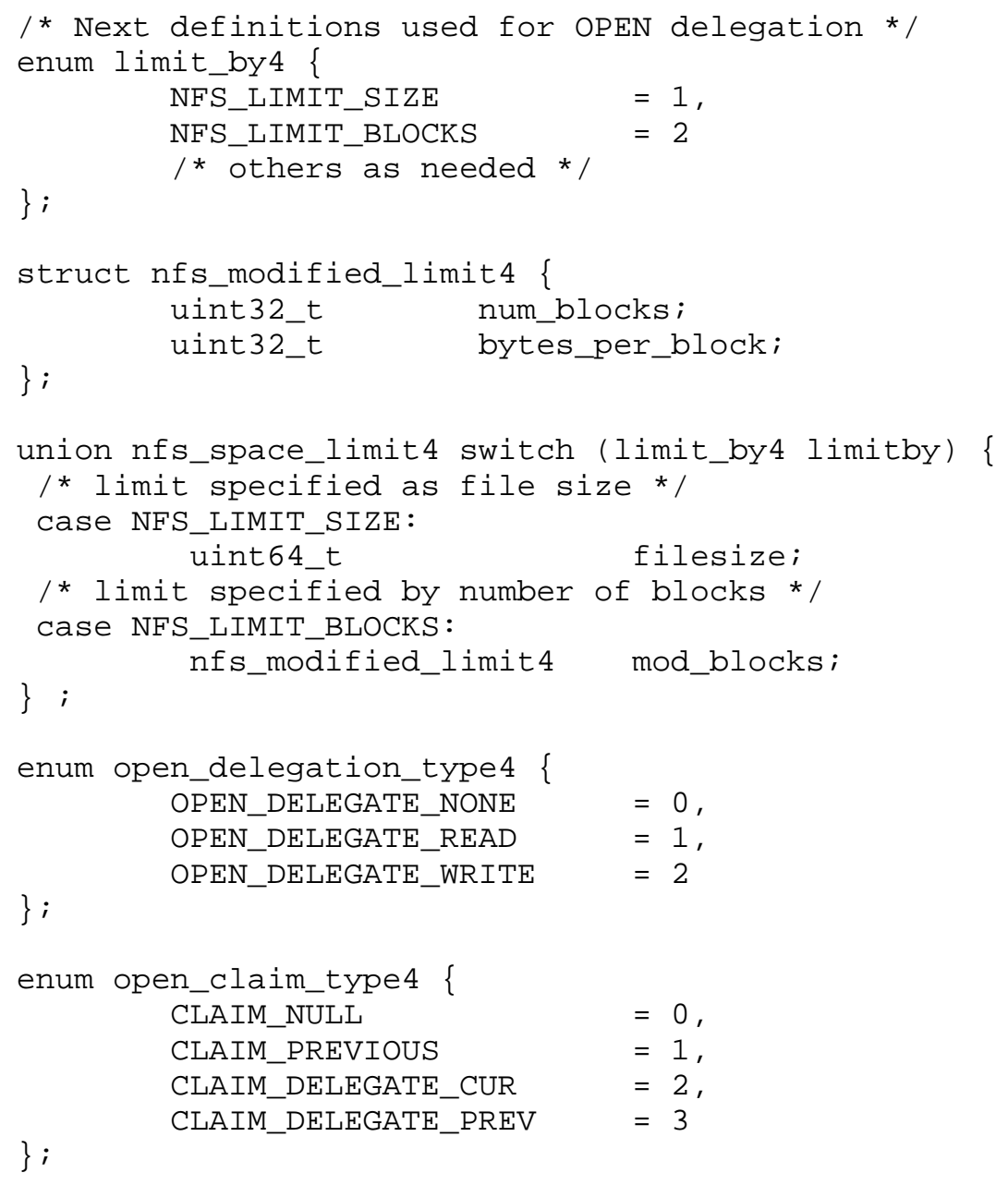




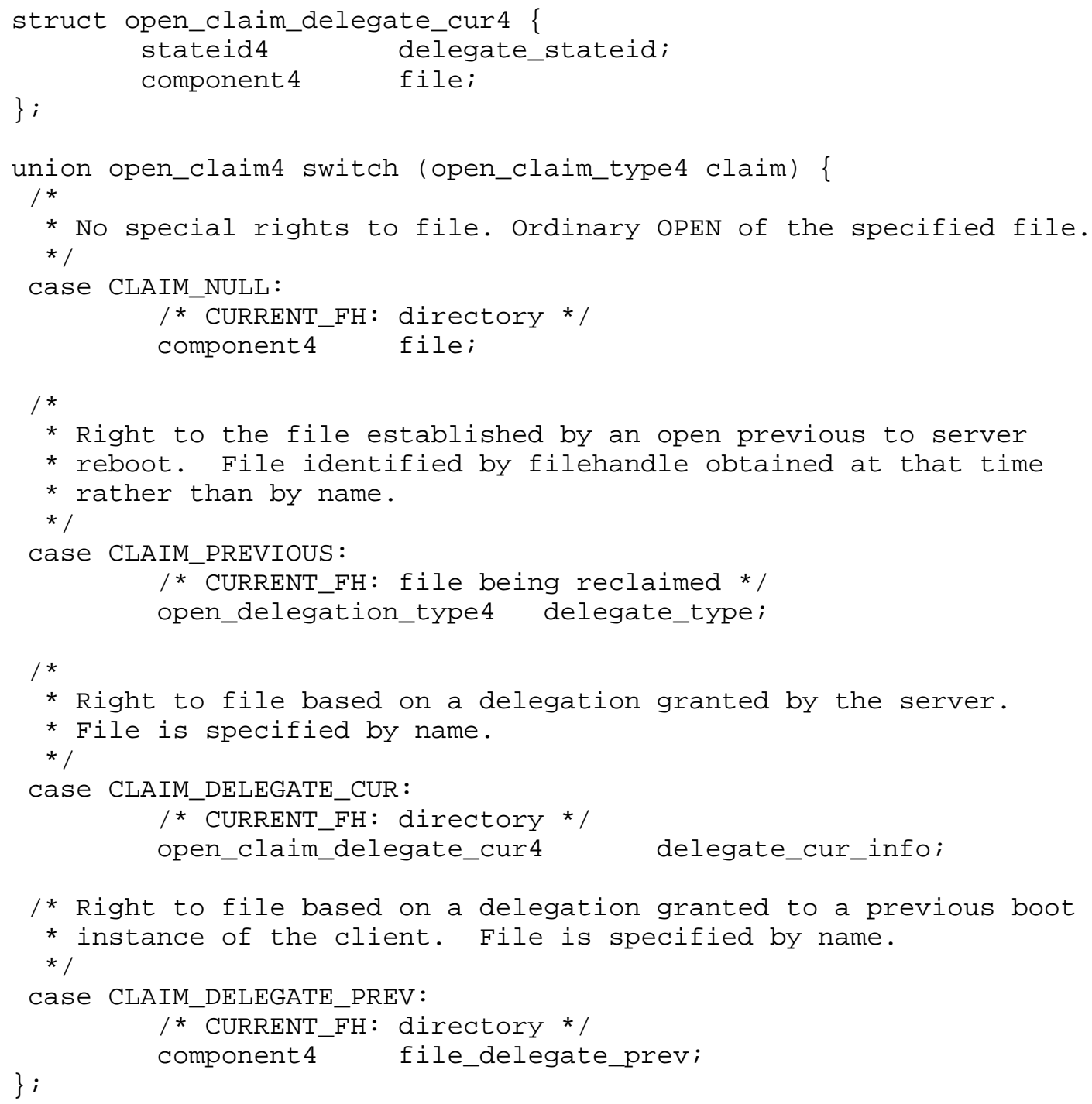


\}

open for read */

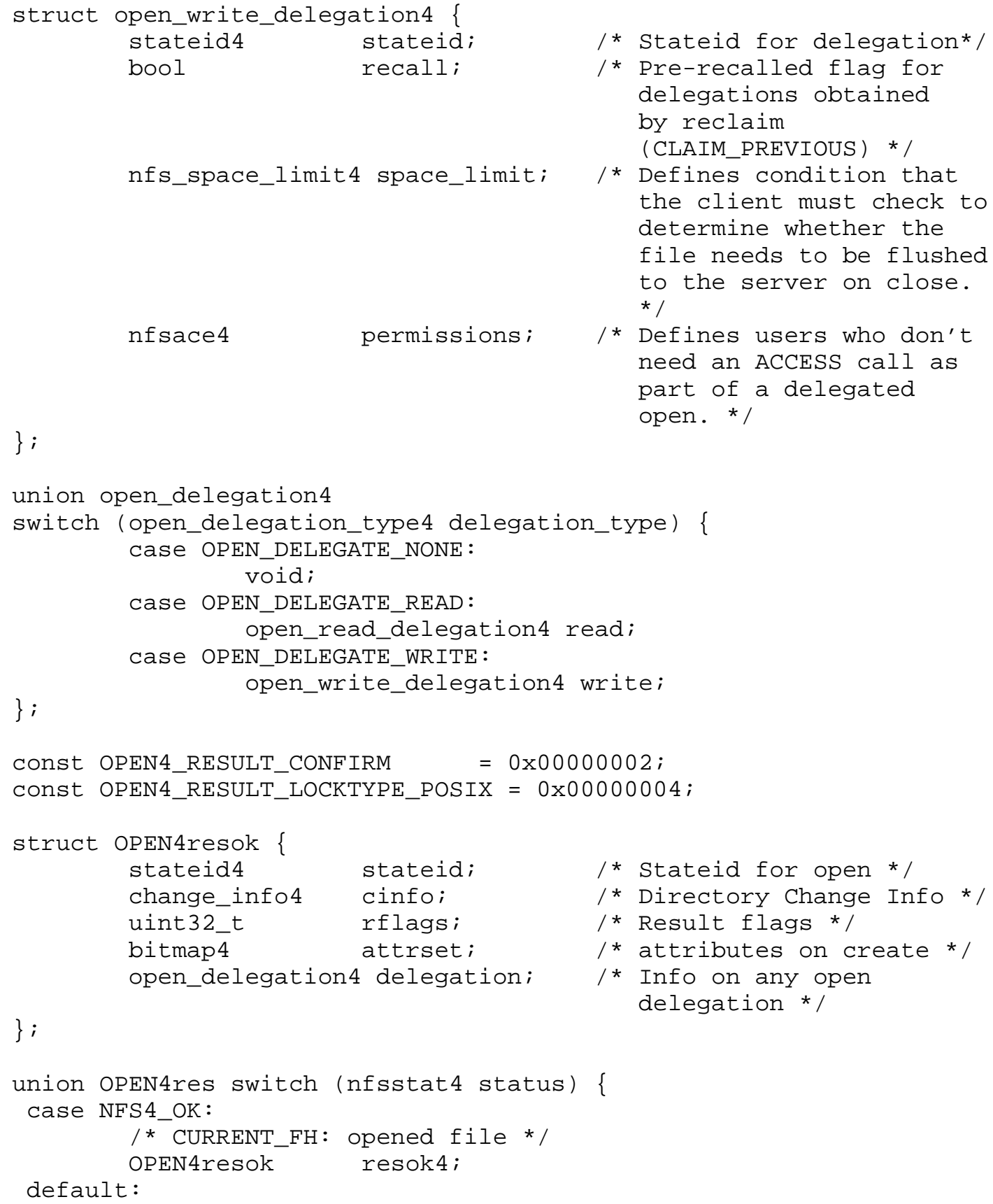




\author{
void; \\ \} ; \\ WARNING TO CLIENT IMPLEMENTORS
}

OPEN resembles LOOKUP in that it generates a filehandle for the client to use. Unlike LOOKUP though, OPEN creates server state on the filehandle. In normal circumstances, the client can only release this state with a CLOSE operation. CLOSE uses the current filehandle to determine which file to close. Therefore the client MUST follow every OPEN operation with a GETFH operation in the same COMPOUND procedure. This will supply the client with the filehandle such that CLOSE can be used appropriately.

Simply waiting for the lease on the file to expire is insufficient because the server may maintain the state indefinitely as long as another client does not attempt to make a conflicting access to the same file.

\title{
DESCRIPTION
}

The OPEN operation creates and/or opens a regular file in a directory with the provided name. If the file does not exist at the server and creation is desired, specification of the method of creation is provided by the openhow parameter. The client has the choice of three creation methods: UNCHECKED, GUARDED, or EXCLUSIVE.

If the current filehandle is a named attribute directory, OPEN will then create or open a named attribute file. Note that exclusive create of a named attribute is not supported. If the createmode is EXCLUSIVE4 and the current filehandle is a named attribute directory, the server will return EINVAL.

UNCHECKED means that the file should be created if a file of that name does not exist and encountering an existing regular file of that name is not an error. For this type of create, createattrs specifies the initial set of attributes for the file. The set of attributes may include any writable attribute valid for regular files. When an UNCHECKED create encounters an existing file, the attributes specified by createattrs are not used, except that when an size of zero is specified, the existing file is truncated. If GUARDED is specified, the server checks for the presence of a duplicate object by name before performing the create. If a duplicate exists, an error of NFS4ERR_EXIST is returned as the status. If the object does not exist, the request is performed as described for UNCHECKED. For 
each of these cases (UNCHECKED and GUARDED) where the operation is successful, the server will return to the client an attribute mask signifying which attributes were successfully set for the object.

EXCLUSIVE specifies that the server is to follow exclusive creation semantics, using the verifier to ensure exclusive creation of the target. The server should check for the presence of a duplicate object by name. If the object does not exist, the server creates the object and stores the verifier with the object. If the object does exist and the stored verifier matches the client provided verifier, the server uses the existing object as the newly created object. If the stored verifier does not match, then an error of NFS4ERR_EXIST is returned. No attributes may be provided in this case, since the server may use an attribute of the target object to store the verifier. If the server uses an attribute to store the exclusive create verifier, it will signify which attribute by setting the appropriate bit in the attribute mask that is returned in the results.

For the target directory, the server returns change_info4 information in cinfo. With the atomic field of the change_info4 struct, the server will indicate if the before and after change attributes were obtained atomically with respect to the link creation.

Upon successful creation, the current filehandle is replaced by that of the new object.

The OPEN operation provides for Windows share reservation capability with the use of the share_access and share_deny fields of the OPEN arguments. The client specifies at OPEN the required share_access and share_deny modes. For clients that do not directly support SHAREs (i.e., UNIX), the expected deny value is DENY_NONE. In the case that there is a existing SHARE reservation that conflicts with the OPEN request, the server returns the error NFS4ERR_SHARE_DENIED. For a complete SHARE request, the client must provide values for the owner and seqid fields for the OPEN argument. For additional discussion of SHARE semantics see the section on 'Share Reservations'.

In the case that the client is recovering state from a server failure, the claim field of the OPEN argument is used to signify that the request is meant to reclaim state previously held.

The "claim" field of the OPEN argument is used to specify the file to be opened and the state information which the client claims to possess. There are four basic claim types which cover the various situations for an OPEN. They are as follows: 
CLAIM_NULL

CLAIM_PREVIOUS

CLAIM_DELEGATE_CUR

CLAIM_DELEGATE_PREV
For the client, this is a new OPEN request and there is no previous state associate with the file for the client.

The client is claiming basic OPEN state for a file that was held previous to a server reboot. Generally used when a server is returning persistent filehandles; the client may not have the file name to reclaim the OPEN.

The client is claiming a delegation for OPEN as granted by the server. Generally this is done as part of recalling a delegation.

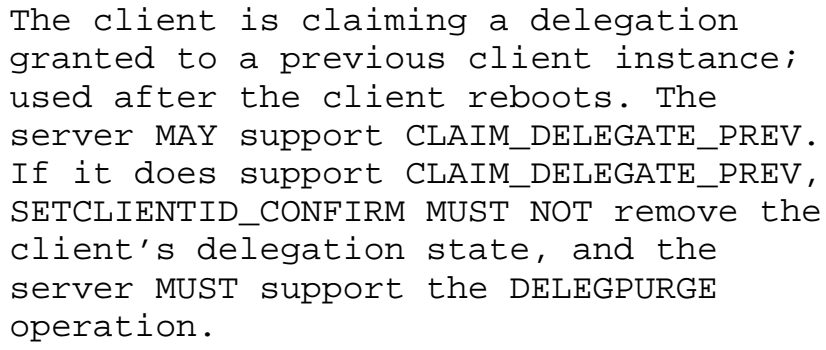

For OPEN requests whose claim type is other than CLAIM_PREVIOUS (i.e., requests other than those devoted to reclaiming opens after a server reboot) that reach the server during its grace or lease expiration period, the server returns an error of NFS4ERR_GRACE.

For any OPEN request, the server may return an open delegation, which allows further opens and closes to be handled locally on the client as described in the section Open Delegation. Note that delegation is up to the server to decide. The client should never assume that delegation will or will not be granted in a particular instance. It should always be prepared for either case. A partial exception is the reclaim (CLAIM_PREVIOUS) case, in which a delegation type is claimed. In this case, delegation will always be granted, although the server may specify an immediate recall in the delegation structure.

The rflags returned by a successful OPEN allow the server to return information governing how the open file is to be handled. 
OPEN4_RESULT_CONFIRM indicates that the client MUST execute an OPEN_CONFIRM operation before using the open file. OPEN4_RESULT_LOCKTYPE_POSIX indicates the server's file locking behavior supports the complete set of Posix locking techniques. From this the client can choose to manage file locking state in a way to handle a mis-match of file locking management.

If the component is of zero length, NFS4ERR_INVAL will be returned. The component is also subject to the normal UTF-8, character support, and name checks. See the section "UTF-8 Related Errors" for further discussion.

When an OPEN is done and the specified lockowner already has the resulting filehandle open, the result is to "OR" together the new share and deny status together with the existing status. In this case, only a single CLOSE need be done, even though multiple OPENs were completed. When such an OPEN is done, checking of share reservations for the new OPEN proceeds normally, with no exception for the existing OPEN held by the same lockowner.

If the underlying filesystem at the server is only accessible in a read-only mode and the OPEN request has specified ACCESS_WRITE or ACCESS_BOTH, the server will return NFS4ERR_ROFS to indicate a readonly filesystem.

As with the CREATE operation, the server MUST derive the owner, owner ACE, group, or group ACE if any of the four attributes are required and supported by the server's filesystem. For an OPEN with the EXCLUSIVE4 createmode, the server has no choice, since such OPEN calls do not include the createattrs field. Conversely, if createattrs is specified, and includes owner or group (or corresponding ACES) that the principal in the RPC call's credentials does not have authorization to create files for, then the server may return NFS4ERR_PERM.

In the case of a OPEN which specifies a size of zero (e.g., truncation) and the file has named attributes, the named attributes are left as is. They are not removed.

\section{IMP LEMENTATION}

The OPEN operation contains support for EXCLUSIVE create. The mechanism is similar to the support in NFS version 3 [RFC1813]. As in NFS version 3, this mechanism provides reliable exclusive creation. Exclusive create is invoked when the how parameter is EXCLUSIVE. In this case, the client provides a verifier that can reasonably be expected to be unique. A combination of a client 
identifier, perhaps the client network address, and a unique number generated by the client, perhaps the RPC transaction identifier, may be appropriate.

If the object does not exist, the server creates the object and stores the verifier in stable storage. For filesystems that do not provide a mechanism for the storage of arbitrary file attributes, the server may use one or more elements of the object meta-data to store the verifier. The verifier must be stored in stable storage to prevent erroneous failure on retransmission of the request. It is assumed that an exclusive create is being performed because exclusive semantics are critical to the application. Because of the expected usage, exclusive CREATE does not rely solely on the normally volatile duplicate request cache for storage of the verifier. The duplicate request cache in volatile storage does not survive a crash and may actually flush on a long network partition, opening failure windows. In the UNIX local filesystem environment, the expected storage location for the verifier on creation is the meta-data (time stamps) of the object. For this reason, an exclusive object create may not include initial attributes because the server would have nowhere to store the verifier.

If the server can not support these exclusive create semantics, possibly because of the requirement to commit the verifier to stable storage, it should fail the OPEN request with the error, NFS 4ERR_NOTSUPP.

During an exclusive CREATE request, if the object already exists, the server reconstructs the object's verifier and compares it with the verifier in the request. If they match, the server treats the request as a success. The request is presumed to be a duplicate of an earlier, successful request for which the reply was lost and that the server duplicate request cache mechanism did not detect. If the verifiers do not match, the request is rejected with the status, NFS 4ERR_EXIST.

Once the client has performed a successful exclusive create, it must issue a SETATTR to set the correct object attributes. Until it does so, it should not rely upon any of the object attributes, since the server implementation may need to overload object meta-data to store the verifier. The subsequent SETATTR must not occur in the same COMPOUND request as the OPEN. This separation will guarantee that the exclusive create mechanism will continue to function properly in the face of retransmission of the request.

Use of the GUARDED attribute does not provide exactly-once semantics. In particular, if a reply is lost and the server does not detect the retransmission of the request, the operation can fail with 
NFS4ERR_EXIST, even though the create was performed successfully. The client would use this behavior in the case that the application has not requested an exclusive create but has asked to have the file truncated when the file is opened. In the case of the client timing out and retransmitting the create request, the client can use GUARDED to prevent against a sequence like: create, write, create (retransmitted) from occurring.

For SHARE reservations, the client must specify a value for share_access that is one of READ, WRITE, or BOTH. For share_deny, the client must specify one of NONE, READ, WRITE, or BOTH. If the client fails to do this, the server must return NFS4ERR_INVAL.

Based on the share_access value (READ, WRITE, or BOTH) the client should check that the requester has the proper access rights to perform the specified operation. This would generally be the results of applying the ACL access rules to the file for the current requester. However, just as with the ACCESS operation, the client should not attempt to second-guess the server's decisions, as access rights may change and may be subject to server administrative controls outside the ACL framework. If the requester is not authorized to READ or WRITE (depending on the share_access value), the server must return NFS4ERR_ACCESS. Note that since the NFS version 4 protocol does not impose any requirement that READs and WRITEs issued for an open file have the same credentials as the OPEN itself, the server still must do appropriate access checking on the READs and WRITEs themselves.

If the component provided to OPEN is a symbolic link, the error NFS4ERR_SYMLINK will be returned to the client. If the current filehandle is not a directory, the error NFS4ERR_NOTDIR will be returned.

\section{ERRORS}

NFS 4ERR_ACCESS

NFS 4ERR_ADMIN_REVOKED

NFS 4ERR_ATTRNOTSUPP

NFS 4ERR_BADCHAR

NF S 4ERR_BADHANDLE

NFS 4ERR_BADNAME

NFS 4ERR_BADOWNER

NFS 4ERR_BAD_SEQID

NFS 4ERR_BADXDR

NF S 4ERR_DELAY

NFS 4ERR_DQUOT

NFS 4ERR_EXIST

NFS 4ERR_EXPIRED 


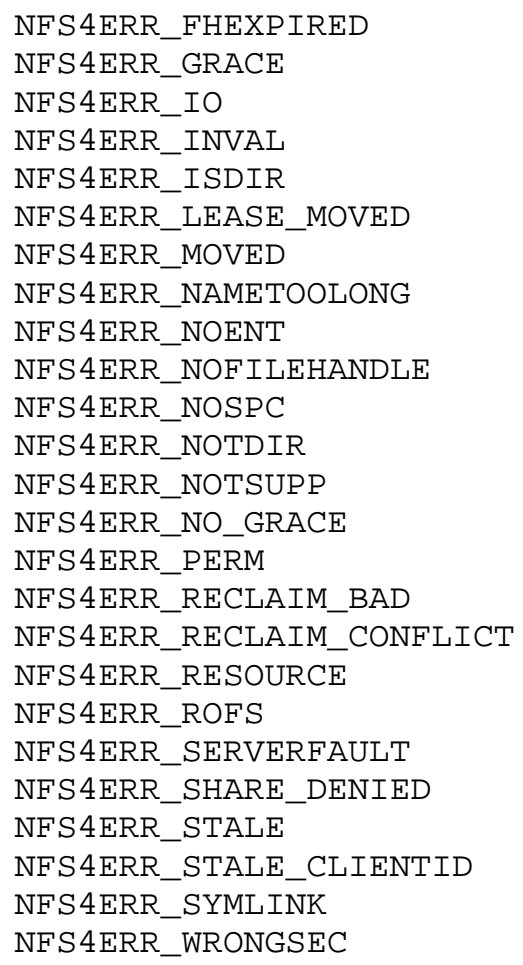

14.2.17. Operation 19: OPENATTR - Open Named Attribute Directory SYNOPSIS

(cfh) createdir $->$ (cfh)

ARGUMENT

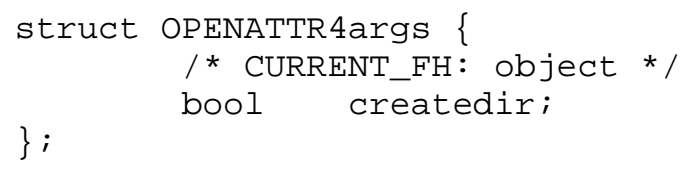




\section{DESCRIPTION}

The OPENATTR operation is used to obtain the filehandle of the named attribute directory associated with the current filehandle. The result of the OPENATTR will be a filehandle to an object of type NF4ATTRDIR. From this filehandle, READDIR and LOOKUP operations can be used to obtain filehandles for the various named attributes associated with the original filesystem object. Filehandles returned within the named attribute directory will have a type of NF 4 NAMEDATTR.

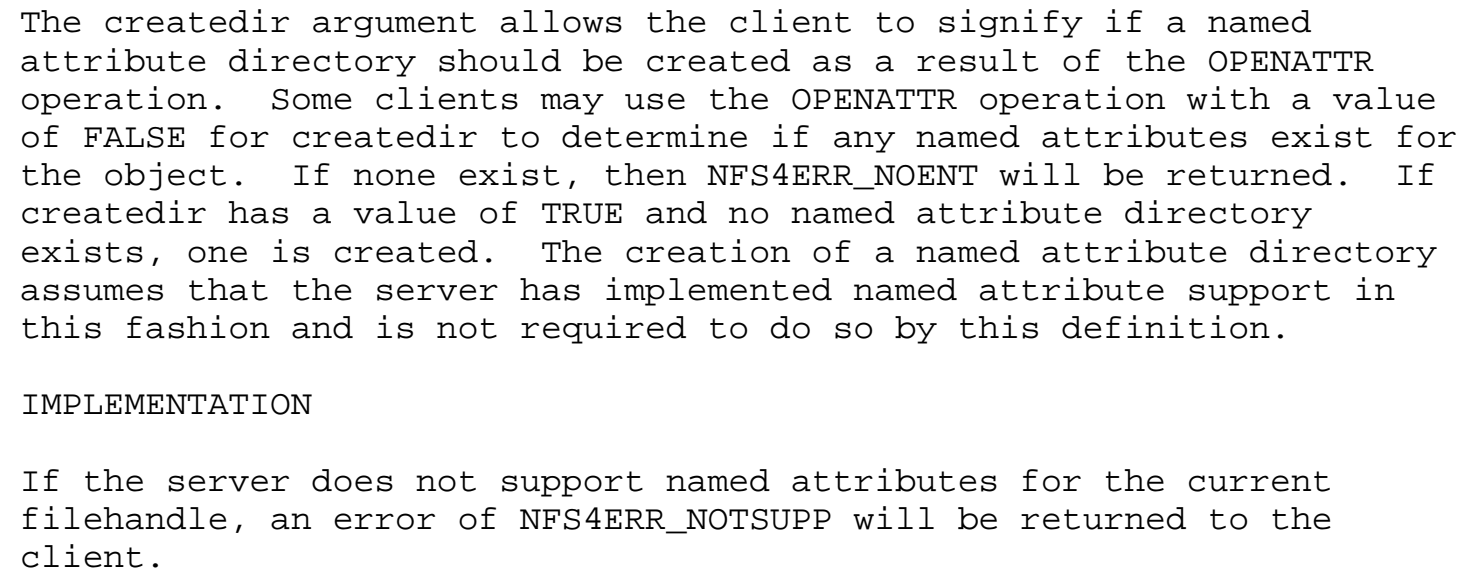

ERRORS

NFS 4ERR_ACCESS
NFS 4ERR_BADHANDLE
NFS 4ERR_BADXDR
NFS 4ERR_DELAY
NES 4ERR_DQUOT
NFS 4ERR_FHEXP IRED
NFS 4ERR_IO
NFS 4ERR_MOVED
NFS 4ERR_NOENT
NFS 4ERR_NOFILEHANDLE
NFS 4ERR_NOSPC
NFS 4ERR_NOTSUPP
NFS 4ERR_RESOURCE
NFS 4ERR_ROFS
NFS 4ERR_SERVERFAULT
NFS 4ERR_STALE


14.2.18. Operation 20: OPEN_CONFIRM - Confirm Open

SYNOPSIS

(cfh), seqid, stateid-> stateid

ARGUMENT

struct OPEN_CONFIRM4args \{

/* CURRENT_FH: opened file */

stateid4 open_stateid;

\} ;

seqid4 seqid;

\section{RESULT}

struct OPEN_CONFIRM4resok \{

\} ;

$$
\text { stateid4 open_stateid; }
$$

union OPEN_CONFIRM4res switch (nfsstat4 status) \{ case NFS4_OK:

default:

OPEN_CONFIRM4resok resok4;

\} ;

void;

\section{DESCRIPTION}

This operation is used to confirm the sequence id usage for the first time that a open_owner is used by a client. The stateid returned from the OPEN operation is used as the argument for this operation along with the next sequence id for the open_owner. The sequence id passed to the OPEN_CONFIRM must be 1 (one) greater than the seqid passed to the OPEN operation from which the open_confirm value was obtained. If the server receives an unexpected sequence id with respect to the original open, then the server assumes that the client will not confirm the original OPEN and all state associated with the original OPEN is released by the server.

On success, the current filehandle retains its value.

IMP LEMENTATION

A given client might generate many open_owner4 data structures for a given clientid. The client will periodically either dispose of its open_owner4s or stop using them for indefinite periods of time. The latter situation is why the NFS version 4 protocol does not have an 
explicit operation to exit an open_owner4: such an operation is of no use in that situation. Instead, to avoid unbounded memory use, the server needs to implement a strategy for disposing of open_owner4s that have no current lock, open, or delegation state for any files and have not been used recently. The time period used to determine when to dispose of open_owner4s is an implementation choice. The time period should certainly be no less than the lease time plus any grace period the server wishes to implement beyond a lease time. The OPEN_CONFIRM operation allows the server to safely dispose of unused open_owner4 data structures.

In the case that a client issues an OPEN operation and the server no longer has a record of the open_owner4, the server needs to ensure that this is a new OPEN and not a replay or retransmission.

Servers must not require confirmation on OPENs that grant delegations or are doing reclaim operations. See section "Use of Open Confirmation" for details. The server can easily avoid this by noting whether it has disposed of one open_owner4 for the given clientid. If the server does not support delegation, it might simply maintain a single bit that notes whether any open_owner4 (for any client) has been disposed of.

The server must hold unconfirmed OPEN state until one of three events occur. First, the client sends an OPEN_CONFIRM request with the appropriate sequence id and stateid within the lease period. In this case, the OPEN state on the server goes to confirmed, and the open_owner4 on the server is fully established.

Second, the client sends another OPEN request with a sequence id that is incorrect for the open_owner4 (out of sequence). In this case, the server assumes the second OPEN request is valid and the first one is a replay. The server cancels the OPEN state of the first OPEN request, establishes an unconfirmed OPEN state for the second OPEN request, and responds to the second OPEN request with an indication that an OPEN_CONFIRM is needed. The process then repeats itself. While there is a potential for a denial of service attack on the client, it is mitigated if the client and server require the use of a security flavor based on Kerberos V5, LIPKEY, or some other flavor that uses cryptography.

What if the server is in the unconfirmed OPEN state for a given open_owner4, and it receives an operation on the open_owner4 that has a stateid but the operation is not OPEN, or it is OPEN_CONFIRM but with the wrong stateid? Then, even if the seqid is correct, the 
server returns NFS4ERR_BAD_STATEID, because the server assumes the operation is a replay: if the server has no established OPEN state, then there is no way, for example, a LoCK operation could be valid.

Third, neither of the two aforementioned events occur for the open_owner4 within the lease period. In this case, the OPEN state is canceled and disposal of the open_owner4 can occur.

\section{ERRORS}

NFS 4ERR_ADMIN_REVOKED
NFS 4ERR_BADHANDLE
NFS 4ERR_BAD_SEQID
NFS 4ERR_BAD_STATEID
NFS 4ERR_BADXDR
NES 4ERR_EXPIRED
NFS 4 ERR_FHEXP IRED
NFS 4ERR_INVAL
NFS 4ERR_ISDIR
NFS 4ERR_MOVED
NFS 4ERR_NOFILEHANDLE
NFS 4ERR_OLD_STATEID
NFS 4ERR_RESOURCE
NFS 4ERR_SERVERFAULT
NFS 4ERR_STALE
NFS 4ERR_STALE_STATEID

14.2.19. Operation 21: OPEN_DOWNGRADE - Reduce Open File Access SYNOPSIS

(cfh), stateid, seqid, access, deny $\rightarrow$ stateid

ARGUMENT

struct OPEN_DOWNGRADE4args \{ /* CURRENT_FH: opened file */ stateid4 open_stateid; seqid4 seqid; uint32_t share_access;

\} ; uint32_t share_deny;

RESULT

struct OPEN_DOWNGRADE4resok \{

\} ;

open_stateid; 


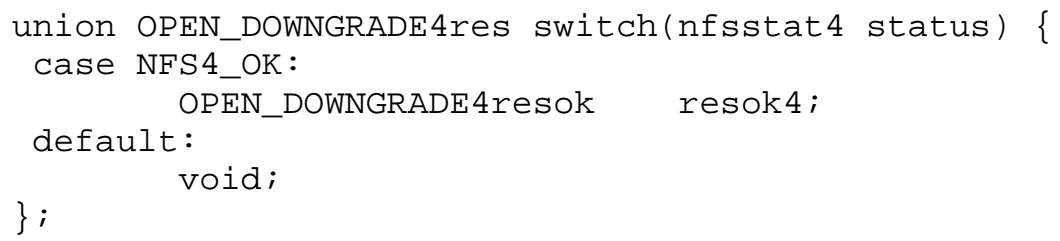
same file multiple times with different share_access and share_deny flags. In this situation, a close of one of the opens may change the appropriate share_access and share_deny flags to remove bits associated with opens no longer in effect.

The share_access and share_deny bits specified in this operation replace the current ones for the specified open file. The share_access and share_deny bits specified must be exactly equal to the union of the share_access and share_deny bits specified for some subset of the OPENs in effect for current openowner on the current file. If that constraint is not respected, the error NFS4ERR_INVAL should be returned. Since share_access and share_deny bits are subsets of those already granted, it is not possible for this request to be denied because of conflicting share reservations.

On success, the current filehandle retains its value.

\section{ERRORS}

NFS 4ERR_ADMIN_REVOKED
NFS 4ERR_BADHANDLE
NFS 4ERR_BAD_SEQID
NFS 4ERR_BAD_STATEID
NFS 4ERR_BADXDR
NFS 4ERR_EXPIRED
NFS 4ERR_EHEXP IRED
NFS 4ERR_INVAL
NFS 4ERR_MOVED
NFS 4ERR_NOFILEHANDLE
NFS 4ERR_OLD_STATEID
NFS 4ERR_RESOURCE
NFS 4ERR_SERVERFAULT
NFS 4ERR_STALE
NFS 4ERR_STALE_STATEID


14.2.20. Operation 22: PUTFH - Set Current Filehandle SYNOPSIS

filehandle $\rightarrow$ (cfh)

ARGUMENT

struct PUTFH4args \{

\} ;

$$
\text { nfs_fh4 object; }
$$

\section{RESULT}

struct PUTFH4res \{ / * CURRENT_FH: */

\} ;

\section{DESCRIPTION}

Replaces the current filehandle with the filehandle provided as an argument.

If the security mechanism used by the requester does not meet the requirements of the filehandle provided to this operation, the server MUST return NFS4ERR_WRONGSEC.

IMP LEMENTATION

Commonly used as the first operator in an NFS request to set the context for following operations.

\section{ERRORS}

NFS SERR_BADHANDLE

NFS 4ERR_BADXDR

NFS 4ERR_FHEXPIRED

NFS 4ERR_MOVED

NFS 4ERR_RESOURCE

NFS 4ERR_SERVERFAULT

NFS 4ERR_STALE

NFS 4ERR_WRONGSEC 
14.2.21. Operation 23: PUTPUBFH - Set Public Filehandle SYNOPSIS

$->(\mathrm{cfh})$

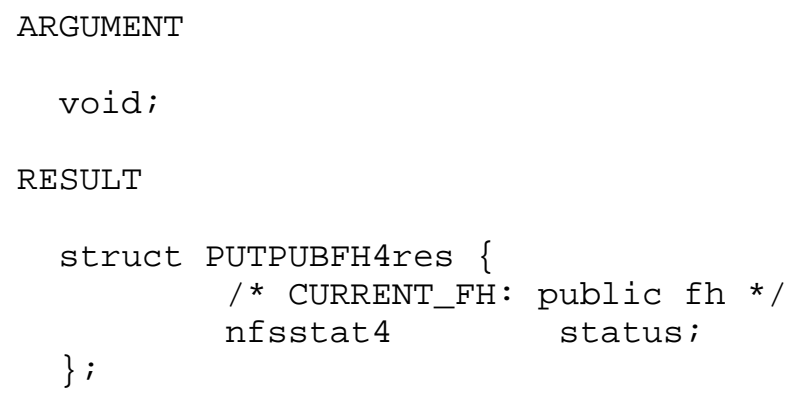




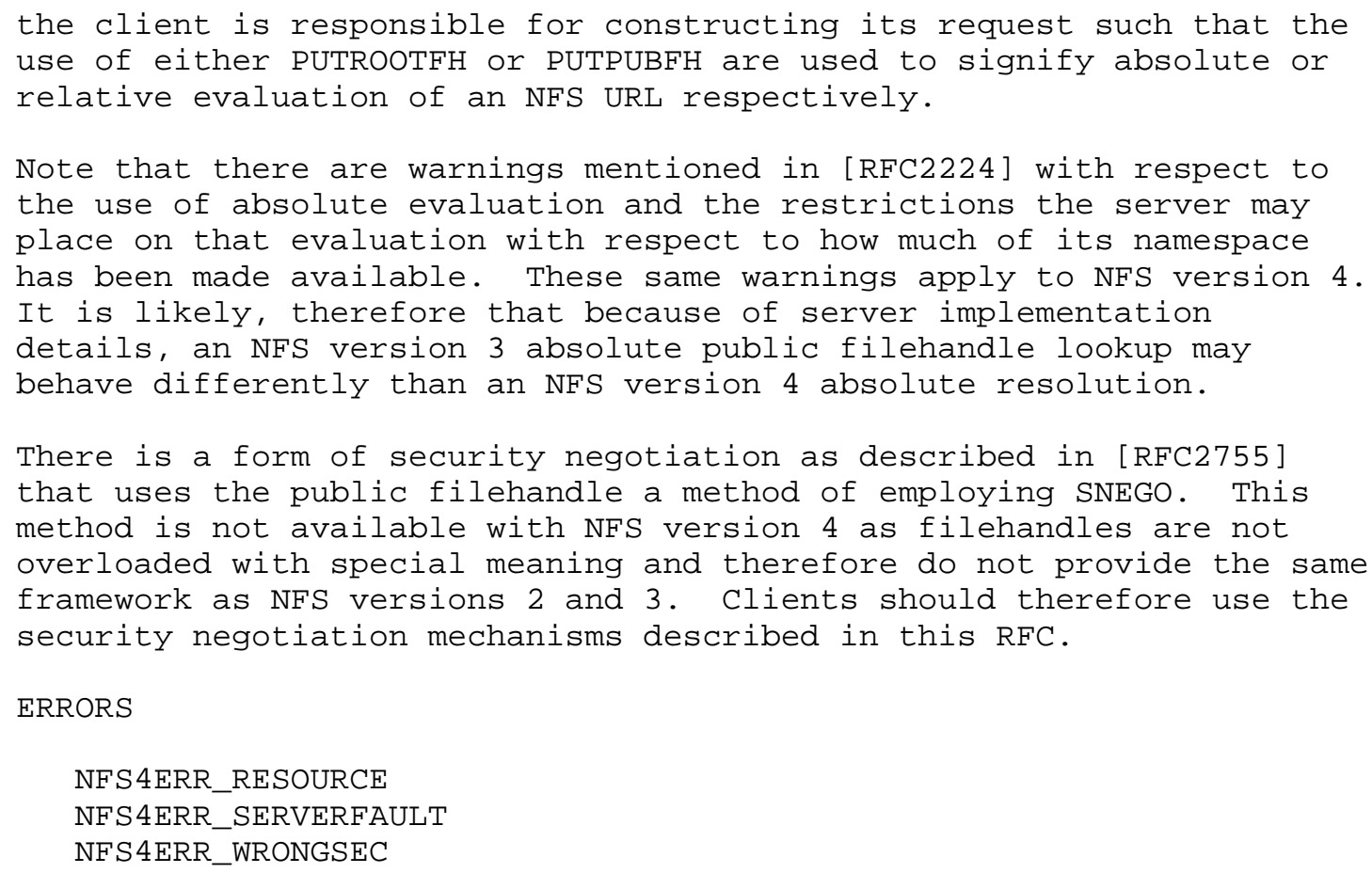




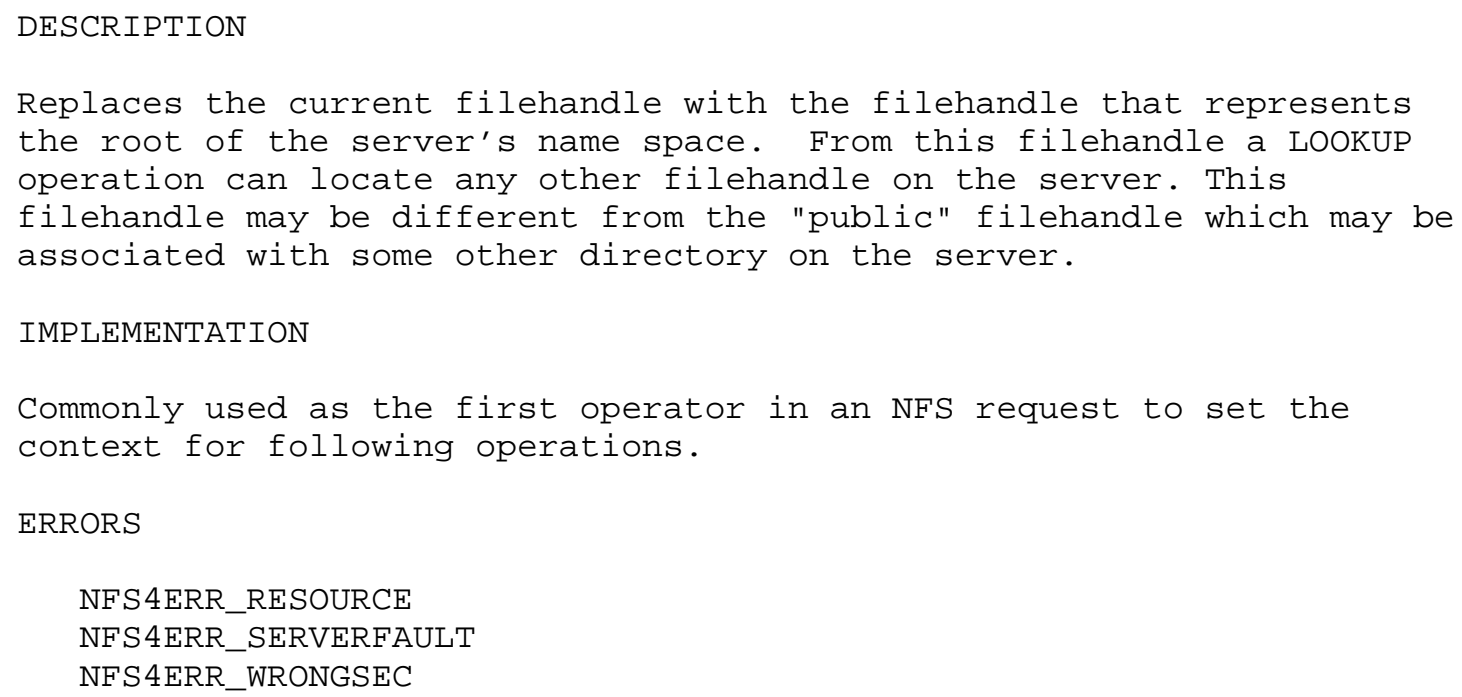




\section{DESCRIPTION}

The READ operation reads data from the regular file identified by the current filehandle.

The client provides an offset of where the READ is to start and a count of how many bytes are to be read. An offset of 0 (zero) means to read data starting at the beginning of the file. If offset is greater than or equal to the size of the file, the status, NFS4_OK, is returned with a data length set to 0 (zero) and eof is set to TRUE. The READ is subject to access permissions checking.

If the client specifies a count value of 0 (zero), the READ succeeds and returns 0 (zero) bytes of data again subject to access permissions checking. The server may choose to return fewer bytes than specified by the client. The client needs to check for this condition and handle the condition appropriately.

The stateid value for a READ request represents a value returned from a previous record lock or share reservation request. The stateid is used by the server to verify that the associated share reservation and any record locks are still valid and to update lease timeouts for the client.

If the read ended at the end-of-file (formally, in a correctly formed $\mathrm{READ}$ request, if offset + count is equal to the size of the file), or the read request extends beyond the size of the file (if offset + count is greater than the size of the file), eof is returned as TRUE; otherwise it is FALSE. A successful READ of an empty file will always return eof as TRUE.

If the current filehandle is not a regular file, an error will be returned to the client. In the case the current filehandle represents a directory, NFS4ERR_ISDIR is return; otherwise, NFS4ERR_INVAL is returned.

For a READ with a stateid value of all bits 0 , the server MAY allow the READ to be serviced subject to mandatory file locks or the current share deny modes for the file. For a READ with a stateid value of all bits 1 , the server MAY allow READ operations to bypass locking checks at the server.

On success, the current filehandle retains its value. 
IMP LEMENTATION

It is possible for the server to return fewer than count bytes of data. If the server returns less than the count requested and eof is set to FALSE, the client should issue another READ to get the remaining data. A server may return less data than requested under several circumstances. The file may have been truncated by another client or perhaps on the server itself, changing the file size from what the requesting client believes to be the case. This would reduce the actual amount of data available to the client. It is possible that the server may back off the transfer size and reduce the read request return. Server resource exhaustion may also occur necessitating a smaller read return.

If mandatory file locking is on for the file, and if the region corresponding to the data to be read from file is write locked by an owner not associated the stateid, the server will return the NFS4ERR_LOCKED error. The client should try to get the appropriate read record lock via the LOCK operation before re-attempting the READ. When the READ completes, the client should release the record lock via LOCKU.

\section{ERRORS}

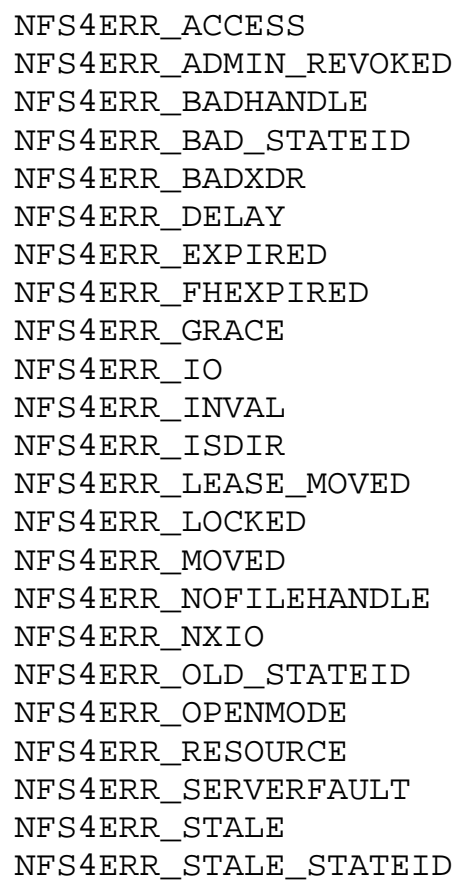




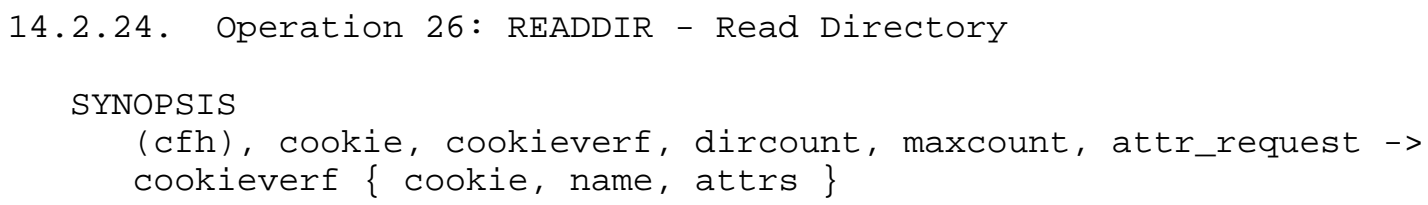




\section{DESCRIPTION}

The READDIR operation retrieves a variable number of entries from a filesystem directory and returns client requested attributes for each entry along with information to allow the client to request additional directory entries in a subsequent READDIR.

The arguments contain a cookie value that represents where the READDIR should start within the directory. A value of 0 (zero) for the cookie is used to start reading at the beginning of the directory. For subsequent READDIR requests, the client specifies a cookie value that is provided by the server on a previous READDIR request.

The cookieverf value should be set to 0 (zero) when the cookie value is 0 (zero) (first directory read). On subsequent requests, it should be a cookieverf as returned by the server. The cookieverf must match that returned by the READDIR in which the cookie was acquired. If the server determines that the cookieverf is no longer valid for the directory, the error NFS4ERR_NOT_SAME must be returned.

The dircount portion of the argument is a hint of the maximum number of bytes of directory information that should be returned. This value represents the length of the names of the directory entries and the cookie value for these entries. This length represents the XDR encoding of the data (names and cookies) and not the length in the native format of the server.

The maxcount value of the argument is the maximum number of bytes for the result. This maximum size represents all of the data being returned within the READDIR4resok structure and includes the XDR overhead. The server may return less data. If the server is unable to return a single directory entry within the maxcount limit, the error NFS4ERR_TOOSMALL will be returned to the client.

Finally, attr_request represents the list of attributes to be returned for each directory entry supplied by the server.

On successful return, the server's response will provide a list of directory entries. Each of these entries contains the name of the directory entry, a cookie value for that entry, and the associated attributes as requested. The "eof" flag has a value of TRUE if there are no more entries in the directory.

The cookie value is only meaningful to the server and is used as a "bookmark" for the directory entry. As mentioned, this cookie is used by the client for subsequent READDIR operations so that it may continue reading a directory. The cookie is similar in concept to a 
READ offset but should not be interpreted as such by the client. Ideally, the cookie value should not change if the directory is modified since the client may be caching these values.

In some cases, the server may encounter an error while obtaining the attributes for a directory entry. Instead of returning an error for the entire READDIR operation, the server can instead return the attribute 'fattr4_rdattr_error'. With this, the server is able to communicate the failure to the client and not fail the entire operation in the instance of what might be a transient failure. Obviously, the client must request the fattr4_rdattr_error attribute for this method to work properly. If the client does not request the attribute, the server has no choice but to return failure for the entire READDIR operation.

For some filesystem environments, the directory entries "." and ".." have special meaning and in other environments, they may not. If the server supports these special entries within a directory, they should not be returned to the client as part of the READDIR response. To enable some client environments, the cookie values of 0,1 , and 2 are to be considered reserved. Note that the UNIX client will use these values when combining the server's response and local representations to enable a fully formed UNIX directory presentation to the application.

For READDIR arguments, cookie values of 1 and 2 should not be used and for READDIR results cookie values of 0,1 , and 2 should not be returned.

On success, the current filehandle retains its value.

\section{IMP LEMENTATION}

The server's filesystem directory representations can differ greatly. A client's programming interfaces may also be bound to the local operating environment in a way that does not translate well into the NFS protocol. Therefore the use of the dircount and maxcount fields are provided to allow the client the ability to provide guidelines to the server. If the client is aggressive about attribute collection during a READDIR, the server has an idea of how to limit the encoded response. The dircount field provides a hint on the number of entries based solely on the names of the directory entries. Since it is a hint, it may be possible that a dircount value is zero. In this case, the server is free to ignore the dircount value and return directory information based on the specified maxcount value. 
The cookieverf may be used by the server to help manage cookie values that may become stale. It should be a rare occurrence that a server is unable to continue properly reading a directory with the provided cookie/cookieverf pair. The server should make every effort to avoid this condition since the application at the client may not be able to properly handle this type of failure.

The use of the cookieverf will also protect the client from using READDIR cookie values that may be stale. For example, if the file system has been migrated, the server may or may not be able to use the same cookie values to service READDIR as the previous server used. With the client providing the cookieverf, the server is able to provide the appropriate response to the client. This prevents the case where the server may accept a cookie value but the underlying directory has changed and the response is invalid from the client's context of its previous READDIR.

Since some servers will not be returning "." and ".." entries as has been done with previous versions of the NFS protocol, the client that requires these entries be present in READDIR responses must fabricate them.

ERRORS

NFS 4ERR_ACCESS
NFS 4ERR_BADHANDLE
NFS 4ERR_BAD_COOKIE
NFS 4ERR_BADXDR
NFS 4ERR_DELAY
NFS 4ERR_FHEXP IRED
NFS 4ERR_INVAL
NFS 4ERR_IO
NFS 4ERR_MOVED
NFS 4ERR_NOFILEHANDLE
NFS 4ERR_NOTDIR
NFS 4ERR_RESOURCE
NFS 4ERR_SERVERFAULT
NFS 4ERR_STALE
NFS 4ERR_TOOSMALL

14.2.25. Operation 27: READLINK - Read Symbolic Link SYNOPSIS $(\mathrm{cfh}) \rightarrow$ linktext 


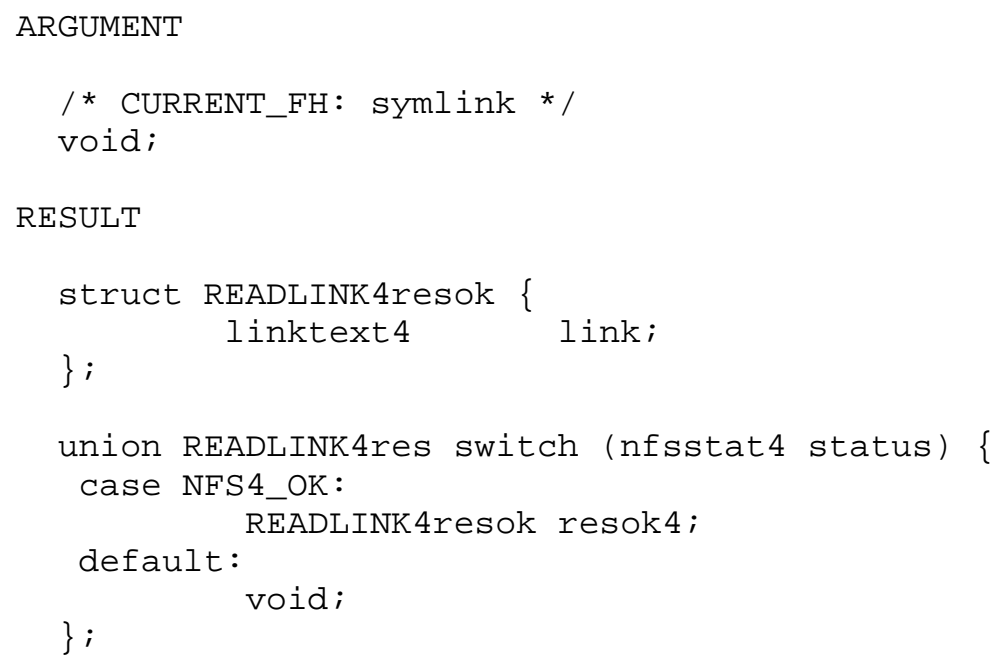




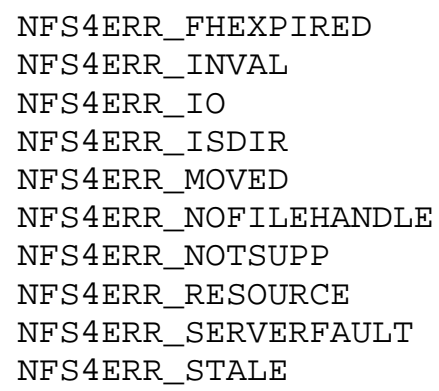

14.2.26. Operation 28: REMOVE - Remove Filesystem Object

SYNOPSIS

(cfh), filename $->$ change_info

ARGUMENT

struct REMOVE4args \{ / CURRENT_FH: directory */

\} ; component 4 target;

RESULT

struct REMOVE4resok \{ \} change_info4 cinfo;

union REMOVE4res switch (nfsstat4 status) \{ case NFS4_OK:

The REMOVE operation removes (deletes) a directory entry named by filename from the directory corresponding to the current filehandle. If the entry in the directory was the last reference to the corresponding filesystem object, the object may be destroyed. 
For the directory where the filename was removed, the server returns change_info4 information in cinfo. With the atomic field of the change_info4 struct, the server will indicate if the before and after change attributes were obtained atomically with respect to the removal.

If the target has a length of 0 (zero), or if target does not obey the UTF-8 definition, the error NFS4ERR_INVAL will be returned.

On success, the current filehandle retains its value.

\section{IMP LEMENTATION}

NFS versions 2 and 3 required a different operator RMDIR for directory removal and REMOVE for non-directory removal. This allowed clients to skip checking the file type when being passed a nondirectory delete system call (e.g., unlink() in POSIX) to remove a directory, as well as the converse (e.g., a rmdir() on a nondirectory) because they knew the server would check the file type. NFS version 4 REMOVE can be used to delete any directory entry independent of its file type. The implementor of an NFS version 4 client's entry points from the unlink() and rmdir() system calls should first check the file type against the types the system call is allowed to remove before issuing a REMOVE. Alternatively, the implementor can produce a COMPOUND call that includes a LOOKUP/VERIFY sequence to verify the file type before a REMOVE operation in the same COMPOUND call.

The concept of last reference is server specific. However, if the numlinks field in the previous attributes of the object had the value 1, the client should not rely on referring to the object via a filehandle. Likewise, the client should not rely on the resources (disk space, directory entry, and so on) formerly associated with the object becoming immediately available. Thus, if a client needs to be able to continue to access a file after using REMOVE to remove it, the client should take steps to make sure that the file will still be accessible. The usual mechanism used is to RENAME the file from its old name to a new hidden name.

If the server finds that the file is still open when the REMOVE arrives:

- The server SHOULD NOT delete the file's directory entry if the file was opened with OPEN4_SHARE_DENY_WRITE or OPEN4_SHARE_DENY_BOTH. 
- If the file was not opened with OPEN4_SHARE_DENY_WRITE or OPEN4_SHARE_DENY_BOTH, the server SHOULD delete the file's directory entry. However, until last CLOSE of the file, the server MAY continue to allow access to the file via its filehandle.

ERRORS

NFS 4ERR_ACCESS
NFS 4ERR_BADCHAR
NFS 4ERR_BADHANDLE
NFS 4ERR_BADNAME
NFS 4ERR_BADXDR
NFS 4ERR_DELAY
NFS 4ERR_FHEXP IRED
NFS 4ERR_EILE_OPEN
NFS 4ERR_INVAL
NFS 4ERR_IO
NFS 4ERR_MOVED
NFS 4ERR_NAMETOOLONG
NFS 4ERR_NOENT
NFS 4ERR_NOFILEHANDLE
NFS 4ERR_NOTDIR
NFS 4ERR_NOTEMPTY
NFS 4ERR_RESOURCE
NFS 4ERR_ROFS
NFS 4ERR_SERVERFAULT
NFS 4ERR_STALE

14.2.27. Operation 29: RENAME - Rename Directory Entry

SYNOPSIS

(sfh), oldname, (cfh), newname $->$ source_change_info, target_change_info

ARGUMENT

struct RENAME4args \{ /* SAVED_FH: source directory */ component 4 oldname; /* CURRENT_FH: target directory * /

\} ; component 4 newname; 
RESULT

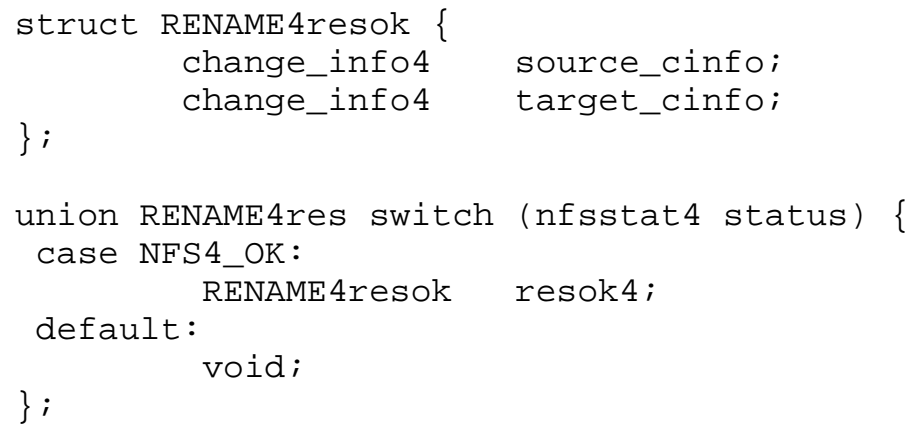

The RENAME operation renames the object identified by oldname in the source directory corresponding to the saved filehandle, as set by the SAVEFH operation, to newname in the target directory corresponding to the current filehandle. The operation is required to be atomic to the client. Source and target directories must reside on the same filesystem on the server. On success, the current filehandle will continue to be the target directory.

If the target directory already contains an entry with the name, newname, the source object must be compatible with the target: either both are non-directories or both are directories and the target must be empty. If compatible, the existing target is removed before the rename occurs (See the IMPLEMENTATION subsection of the section "Operation 28: REMOVE - Remove Filesystem Object" for client and server actions whenever a target is removed). If they are not compatible or if the target is a directory but not empty, the server will return the error, NFS4ERR_EXIST.

If oldname and newname both refer to the same file (they might be hard links of each other), then RENAME should perform no action and return success.

For both directories involved in the RENAME, the server returns change_info4 information. With the atomic field of the change_info4 struct, the server will indicate if the before and after change attributes were obtained atomically with respect to the rename.

If the oldname refers to a named attribute and the saved and current filehandles refer to different filesystem objects, the server will return NFS4ERR_XDEV just as if the saved and current filehandles represented directories on different filesystems. 
If the oldname or newname has a length of 0 (zero), or if oldname or newname does not obey the UTF-8 definition, the error NFS4ERR_INVAL will be returned.

\section{IMP LEMENTATION}

The RENAME operation must be atomic to the client. The statement "source and target directories must reside on the same filesystem on the server" means that the fsid fields in the attributes for the directories are the same. If they reside on different filesystems, the error, NFS4ERR_XDEV, is returned.

Based on the value of the fh_expire_type attribute for the object, the filehandle may or may not expire on a RENAME. However, server implementors are strongly encouraged to attempt to keep filehandles from expiring in this fashion.

On some servers, the file names "." and ".." are illegal as either oldname or newname, and will result in the error NFS4ERR_BADNAME. In addition, on many servers the case of oldname or newname being an alias for the source directory will be checked for. Such servers will return the error NFS4ERR_INVAL in these cases.

If either of the source or target filehandles are not directories, the server will return NFS4ERR_NOTDIR.

\section{ERRORS}

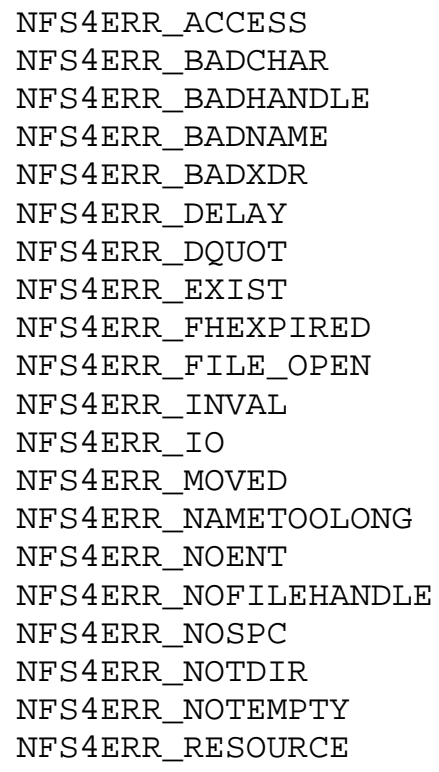




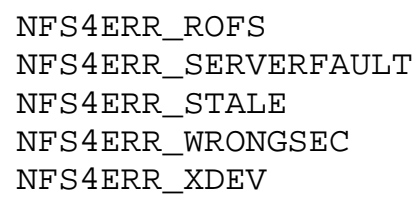

14.2.28. Operation 30: RENEW - Renew a Lease

SYNOPSIS

clientid $\rightarrow()$

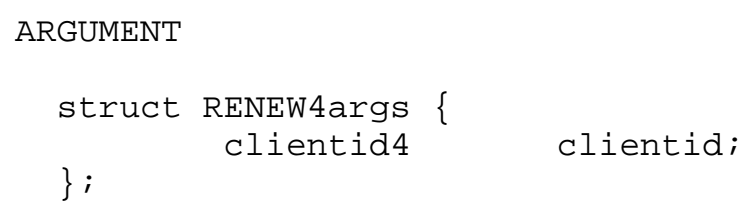


The client that issues RENEW MUST choose the principal, RPC security flavor, and if applicable, GSS-API mechanism and service via one of the following algorithms:

- The client uses the same principal, RPC security flavor -- and if the flavor was RPCSEC_GSS -- the same mechanism and service that was used when the client id was established via SETCLIENTID_CONFIRM.

- The client uses any principal, RPC security flavor mechanism and service combination that currently has an OPEN file on the server. I.e., the same principal had a successful OPEN operation, the file is still open by that principal, and the flavor, mechanism, and service of RENEW match that of the previous OPEN.

The server MUST reject a RENEW that does not use one the aforementioned algorithms, with the error NFS4ERR_ACCESS.

ERRORS

NFS 4ERR_ACCESS
NFS 4 ERR_ADMIN_REVOKED
NFS 4 ERR_BADXDR
NFS 4 ERR_CB_PATH_DOWN
NES 4ERR_EXPIRED
NFS 4ERR_LEASE_MOVED
NFS 4 ERR_RESOURCE
NFS 4 ERR_SERVERFAULT
NFS 4ERR_STALE_CLIENTID

14.2.29. Operation 31: RESTOREFH - Restore Saved Filehandle SYNOPSIS

$(\mathrm{sfh})->(\mathrm{cfh})$

ARGUMENT

/* SAVED_FH: */

void;

RESULT

struct RESTOREFH4res \{ /* CURRENT_FH: value of saved fh */

\} ; nfsstat4 status;

Shepler, et al. Standards Track

[Page 201] 
DESCRIPTION

Set the current filehandle to the value in the saved filehandle. If there is no saved filehandle then return the error NFS4ERR_RESTOREFH.

IMP LEMENTATION

Operations like OPEN and LOOKUP use the current filehandle to represent a directory and replace it with a new filehandle. Assuming the previous filehandle was saved with a SAVEFH operator, the previous filehandle can be restored as the current filehandle. This is commonly used to obtain post-operation attributes for the directory, e.g.,

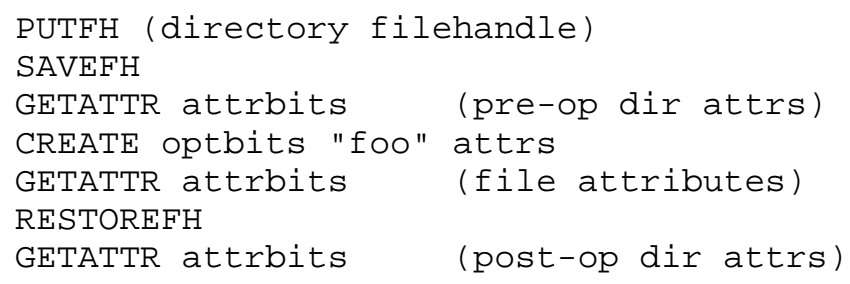

ERRORS

NFS 4ERR_BADHANDLE
NFS 4ERR_FHEXP IRED
NFS 4ERR_MOVED
NFS 4ERR_RESOURCE
NFS 4ERR_RESTOREFH
NFS 4ERR_SERVERFAULT
NFS 4ERR_STALE
NFS 4ERR_WRONGSEC

14.2.30. Operation 32: SAVEFH - Save Current Filehandle SYNOPSIS

$(\mathrm{cfh})->(\mathrm{sfh})$

ARGUMENT

$/$ *URRENT_FH: */ void; 
RESULT

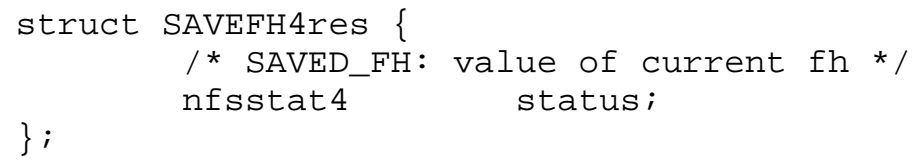

Shepler, et al. Standards Track [Page 203] 


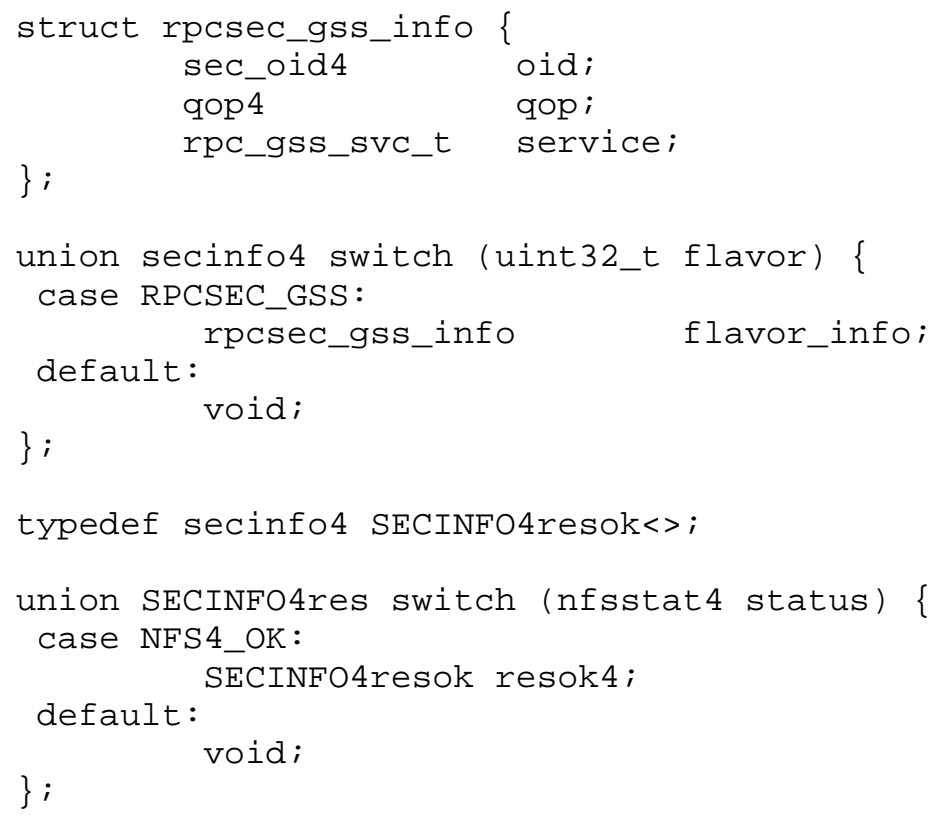


On success, the current filehandle retains its value.

If the name has a length of 0 (zero), or if name does not obey the UTF-8 definition, the error NFS4ERR_INVAL will be returned.

\section{IMP LEMENTATION}

The SECINFO operation is expected to be used by the NFS client when the error value of NFS4ERR_WRONGSEC is returned from another NFS operation. This signifies to the client that the server's security policy is different from what the client is currently using. At this point, the client is expected to obtain a list of possible security flavors and choose what best suits its policies.

As mentioned, the server's security policies will determine when a client request receives NFS4ERR_WRONGSEC. The operations which may receive this error are: LINK, LOOKUP, OPEN, PUTFH, PUTPUBFH, PUTROOTFH, RESTOREFH, RENAME, and indirectly READDIR. LINK and RENAME will only receive this error if the security used for the operation is inappropriate for saved filehandle. With the exception of READDIR, these operations represent the point at which the client can instantiate a filehandle into the "current filehandle" at the server. The filehandle is either provided by the client (PUTFH, PUTPUBFH, PUTROOTFH) or generated as a result of a name to filehandle translation (LOOKUP and OPEN). RESTOREFH is different because the filehandle is a result of a previous SAVEFH. Even though the filehandle, for RESTOREFH, might have previously passed the server's inspection for a security match, the server will check it again on RESTOREFH to ensure that the security policy has not changed.

If the client wants to resolve an error return of NFS4ERR_WRONGSEC, the following will occur:

- For LOOKUP and OPEN, the client will use SECINFO with the same current filehandle and name as provided in the original LookUP or OPEN to enumerate the available security triples.

o For LINK, PUTFH, RENAME, and RESTOREFH, the client will use SECINFO and provide the parent directory filehandle and object name which corresponds to the filehandle originally provided by the PUTFH RESTOREFH, or for LINK and RENAME, the SAVEFH.

- For PUTROOTFH and PUTPUBFH, the client will be unable to use the SECINFO operation since SECINFO requires a current filehandle and none exist for these two operations. Therefore, the client must iterate through the security triples available at the client and reattempt the PUTROOTFH or PUTPUBFH operation. In the unfortunate event none of the MANDATORY security triples are supported by the 
client and server, the client SHOULD try using others that support integrity. Failing that, the client can try using AUTH_NONE, but because such forms lack integrity checks, this puts the client at risk. Nonetheless, the server SHOULD allow the client to use whatever security form the client requests and the server supports, since the risks of doing so are on the client.

The READDIR operation will not directly return the NFS4ERR_WRONGSEC error. However, if the READDIR request included a request for attributes, it is possible that the READDIR request's security triple does not match that of a directory entry. If this is the case and the client has requested the rdattr_error attribute, the server will return the NFS4ERR_WRONGSEC error in rdattr_error for the entry.

See the section "Security Considerations" for a discussion on the recommendations for security flavor used by SECINFO.

\section{ERRORS}

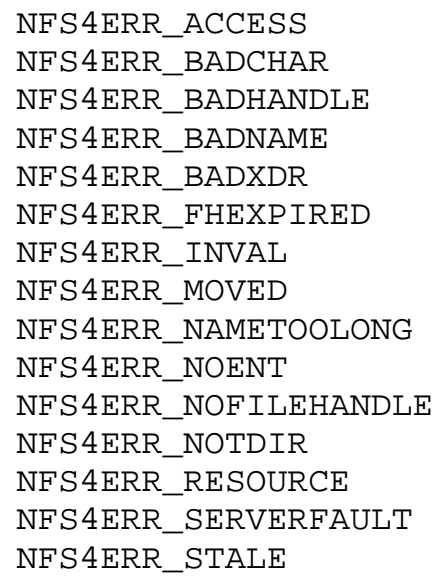

14.2.32. Operation 34: SETATTR - Set Attributes

\section{SYNOPSIS}

(cfh), stateid, attrmask, attr_vals $->$ attrsset

\section{ARGUMENT}

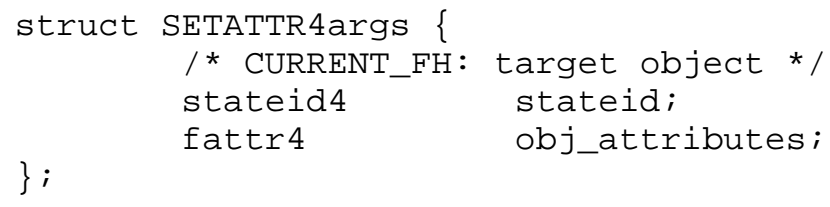


RESULT

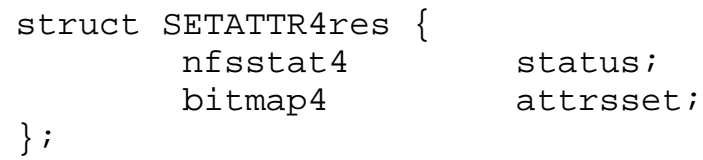


the end of the file to be discarded, and a size greater than the current size of the file causes logically zeroed data bytes to be added to the end of the file. Servers are free to implement this using holes or actual zero data bytes. Clients should not make any assumptions regarding a server's implementation of this feature, beyond that the bytes returned will be zeroed. Servers must support extending the file size via SETATTR.

SETATTR is not guaranteed atomic. A failed SETATTR may partially change a file's attributes.

Changing the size of a file with SETATTR indirectly changes the time_modify. A client must account for this as size changes can result in data deletion.

The attributes time_access_set and time_modify_set are write-only attributes constructed as a switched union so the client can direct the server in setting the time values. If the switched union specifies SET_TO_CLIENT_TIME4, the client has provided an nfstime4 to be used for the operation. If the switch union does not specify SET_TO_CLIENT_TIME4, the server is to use its current time for the SETATTR operation.

If server and client times differ, programs that compare client time to file times can break. A time maintenance protocol should be used to limit client/server time skew.

Use of a COMPOUND containing a VERIFY operation specifying only the change attribute, immediately followed by a SETATTR, provides a means whereby a client may specify a request that emulates the functionality of the SETATTR guard mechanism of NFS version 3 . Since the function of the guard mechanism is to avoid changes to the file attributes based on stale information, delays between checking of the guard condition and the setting of the attributes have the potential to compromise this function, as would the corresponding delay in the NFS version 4 emulation. Therefore, NFS version 4 servers should take care to avoid such delays, to the degree possible, when executing such a request.

If the server does not support an attribute as requested by the client, the server should return NFS4ERR_ATTRNOTSUPP.

A mask of the attributes actually set is returned by SETATTR in all cases. That mask must not include attributes bits not requested to be set by the client, and must be equal to the mask of attributes requested to be set only if the SETATTR completes without error. 


\section{ERRORS}

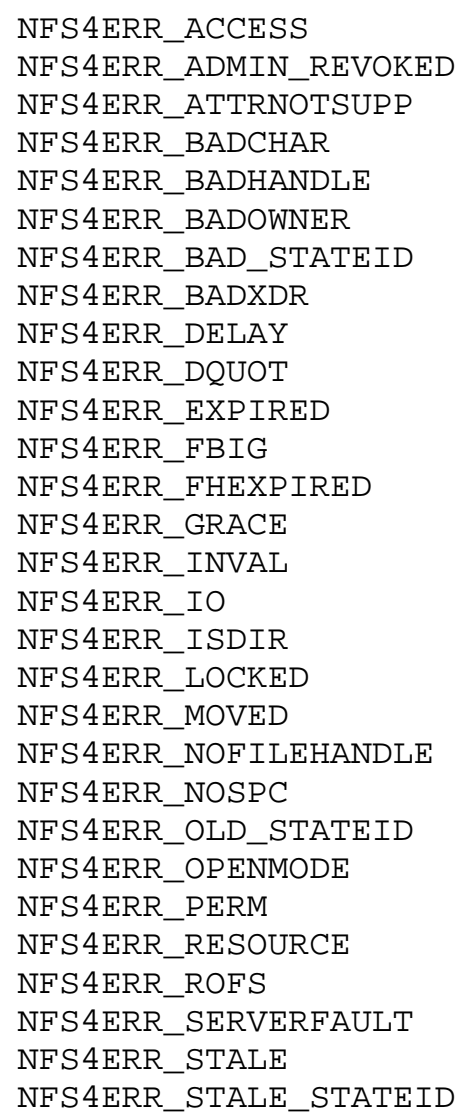

14.2.33. Operation 35: SETCLIENTID - Negotiate Clientid

SYNOPSIS

client, callback, callback_ident -> clientid, setclientid_confirm ARGUMENT

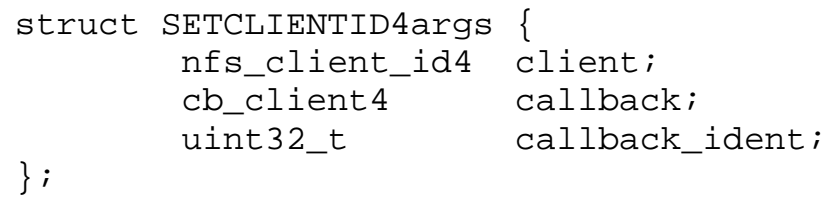




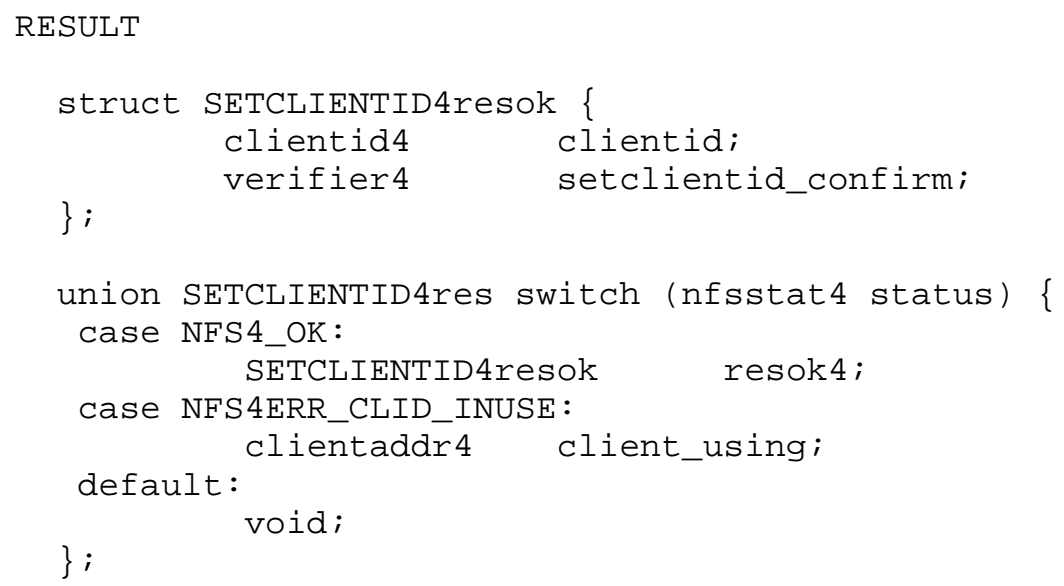




\section{IMP LEMENTATION}

To understand how to implement SETCLIENTID, make the following notations. Let:

$\mathrm{x}$ be the value of the client.id subfield of the SETCLIENTID4args structure.

$\mathrm{V}$ be the value of the client.verifier subfield of the SETCLIENTID4args structure.

$C$ be the value of the clientid field returned in the SETCLIENTID4resok structure.

$\mathrm{k}$ represent the value combination of the fields callback and callback_ident fields of the SETCLIENTID4args structure.

$\mathrm{s}$ be the setclientid_confirm value returned in the SETCLIENTID4resok structure.

$\{\mathrm{v}, \mathrm{x}, \mathrm{C}, \mathrm{k}, \mathrm{s}\}$ be a quintuple for a client record. A client record is confirmed if there has been a SETCLIENTID_CONFIRM operation to confirm it. Otherwise it is unconfirmed. An unconfirmed record is established by a SETCLIENTID call.

Since SETCLIENTID is a non-idempotent operation, let us assume that the server is implementing the duplicate request cache (DRC).

When the server gets a SETCLIENTID $\{v, x, k\}$ request, it processes it in the following manner.

- It first looks up the request in the DRC. If there is a hit, it returns the result cached in the DRC. The server does NOT remove client state (locks, shares, delegations) nor does it modify any recorded callback and callback_ident information for client $\{\mathrm{x}\}$.

For any DRC miss, the server takes the client id string $x$, and searches for client records for $x$ that the server may have recorded from previous SETCLIENTID calls. For any confirmed record with the same id string $x$, if the recorded principal does not match that of SETCLIENTID call, then the server returns a NFS4ERR_CLID_INUSE error.

For brevity of discussion, the remaining description of the processing assumes that there was a DRC miss, and that where the server has previously recorded a confirmed record for client $x$, the aforementioned principal check has successfully passed. 
- The server checks if it has recorded a confirmed record for $\{v$, $\mathrm{x}, \mathrm{c}, \mathrm{l}, \mathrm{s}\}$, where $\mathrm{l}$ may or may not equal k. If so, and since the id verifier $v$ of the request matches that which is confirmed and recorded, the server treats this as a probable callback information update and records an unconfirmed $\{v, x, c, k, t\}$ and leaves the confirmed $\{\mathrm{V}, \mathrm{x}, \mathrm{C}, \mathrm{l}, \mathrm{s}\}$ in place, such that $t$ $!=s$. It does not matter if $\mathrm{k}$ equals $l$ or not. Any pre-existing unconfirmed $\{\mathrm{V}, \mathrm{X}, \mathrm{C}, \star, \star\}$ is removed.

The server returns $\{c, t\}$. It is indeed returning the old clientid4 value $c$, because the client apparently only wants to update callback value $\mathrm{k}$ to value 1 . It's possible this request is one from the Byzantine router that has stale callback information, but this is not a problem. The callback information update is only confirmed if followed up by a SETCLIENTID_CONFIRM $\{c, t\}$.

The server awaits confirmation of $\mathrm{k}$ via SETCLIENTID_CONFIRM $\{c, t\}$.

The server does NOT remove client (lock/share/delegation) state for $x$.

- The server has previously recorded a confirmed $\{u, x, c, l, s\}$ record such that $\mathrm{v} !=\mathrm{u}, \mathrm{l}$ may or may not equal $\mathrm{k}$, and has not recorded any unconfirmed $\{\star, x, *, *, *\}$ record for $x$. The server records an unconfirmed $\{\mathrm{v}, \mathrm{x}, \mathrm{d}, \mathrm{k}, \mathrm{t}\}(\mathrm{d} !=\mathrm{c}, \mathrm{t} !=\mathrm{s})$.

The server returns $\{d, t\}$.

The server awaits confirmation of $\{d, k\}$ via SETCLIENTID_CONFIRM $\{d, t\}$.

The server does NOT remove client (lock/share/delegation) state for $x$.

- The server has previously recorded a confirmed $\{u, x, c, 1, s\}$ record such that $\mathrm{v} !=\mathrm{u}$, 1 may or may not equal $\mathrm{k}$, and recorded an unconfirmed $\{\mathrm{w}, \mathrm{x}, \mathrm{d}, \mathrm{m}, \mathrm{t}\}$ record such that $\mathrm{c} !=\mathrm{d}, \mathrm{t} !=\mathrm{s}, \mathrm{m}$ may or may not equal k, m may or may not equal 1 , and $k$ may or may not equal 1 . Whether $\mathrm{w}==\mathrm{v}$ or $\mathrm{w} !=\mathrm{v}$ makes no difference. The server simply removes the unconfirmed $\{\mathrm{w}, \mathrm{x}, \mathrm{d}, \mathrm{m}, \mathrm{t}\}$ record and replaces it with an unconfirmed $\{v, x, e, k, r\}$ record, such that $e !=d, e !=c, r !=t, r !=s$.

The server returns $\{e, r\}$.

The server awaits confirmation of $\{e, k\}$ via SETCLIENTID_CONFIRM $\{e, r\}$. 
The server does NOT remove client (lock/share/delegation) state for $x$.

o The server has no confirmed $\{*, x, *, *, *\}$ for $x$. It may or may not have recorded an unconfirmed $\{u, x, c, l, s\}$, where $l$ may or may not equal $k$, and $u$ may or may not equal $v$. Any unconfirmed record $\{\mathrm{u}, \mathrm{x}, \mathrm{c}, \mathrm{l}, \star\}$, regardless whether $\mathrm{u}==\mathrm{v}$ or $\mathrm{l}=\mathrm{k}$, is replaced with an unconfirmed record $\{v, x, d, k, t\}$ where $d !=$ $c, t !=s$.

The server returns $\{d, t\}$.

The server awaits confirmation of $\{d, k\}$ via SETCLIENTID_CONFIRM $\{d, t\}$. The server does NOT remove client (lock/share/delegation) state for $\mathrm{x}$.

The server generates the clientid and setclientid_confirm values and must take care to ensure that these values are extremely unlikely to ever be regenerated.

\section{ERRORS}

NFS 4ERR_BADXDR
NFS 4ERR_CLID_INUSE
NFS 4ERR_INVAL
NFS 4ERR_RESOURCE
NFS 4ERR_SERVERFAULT

14.2.34. Operation 36: SETCLIENTID_CONFIRM - Confirm Clientid SYNOPSIS

clientid, verifier $->-$

ARGUMENT

$\begin{array}{cc}\text { struct } & \text { SETCLIENTID_CONFIRM4args }\{ \\ \text { clientid4 } & \text { clientid; } \\ \text { verifier4 } & \text { setclientid_confirm; }\end{array}$

\} ;

RESULT

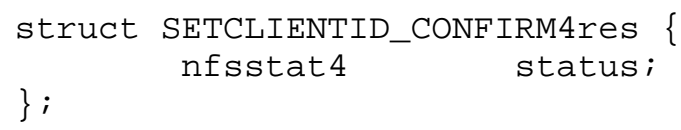




\section{DESCRIPTION}

This operation is used by the client to confirm the results from a previous call to SETCLIENTID. The client provides the server supplied (from a SETCLIENTID response) clientid. The server responds with a simple status of success or failure.

\section{IMP LEMENTATION}

The client must use the SETCLIENTID_CONFIRM operation to confirm the following two distinct cases:

- The client's use of a new shorthand client identifier (as returned from the server in the response to SETCLIENTID), a new callback value (as specified in the arguments to SETCLIENTID) and a new callback_ident (as specified in the arguments to SETCLIENTID) value. The client's use of SETCLIENTID_CONFIRM in this case also confirms the removal of any of the client's previous relevant leased state. Relevant leased client state includes record locks, share reservations, and where the server does not support the CLAIM_DELEGATE_PREV claim type, delegations. If the server supports CLAIM_DELEGATE_PREV, then SETCLIENTID_CONFIRM MUST NOT remove delegations for this client; relevant leased client state would then just include record locks and share reservations.

- The client's re-use of an old, previously confirmed, shorthand client identifier, a new callback value, and a new callback_ident value. The client's use of SETCLIENTID_CONFIRM in this case MUST NOT result in the removal of any previous leased state (locks, share reservations, and delegations)

We use the same notation and definitions for $\mathrm{v}, \mathrm{x}, \mathrm{c}, \mathrm{k}, \mathrm{s}$, and unconfirmed and confirmed client records as introduced in the description of the SETCLIENTID operation. The arguments to SETCLIENTID_CONFIRM are indicated by the notation $\{\mathrm{c}, \mathrm{s}\}$, where $\mathrm{C}$ is a value of type clientid4, and $s$ is a value of type verifier4 corresponding to the setclientid_confirm field.

As with SETCLIENTID, SETCLIENTID_CONFIRM is a non-idempotent operation, and we assume that the server is implementing the duplicate request cache (DRC).

When the server gets a SETCLIENTID_CONFIRM $\{c, s\}$ request, it processes it in the following manner. 
o It first looks up the request in the DRC. If there is a hit, it returns the result cached in the DRC. The server does not remove any relevant leased client state nor does it modify any recorded callback and callback_ident information for client $\{x\}$ as represented by the shorthand value $\mathrm{c}$.

For a DRC miss, the server checks for client records that match the shorthand value $c$. The processing cases are as follows:

- The server has recorded an unconfirmed $\{\mathrm{V}, \mathrm{x}, \mathrm{C}, \mathrm{k}, \mathrm{s}\}$ record and a confirmed $\{\mathrm{V}, \mathrm{x}, \mathrm{c}, \mathrm{l}, \mathrm{t}\}$ record, such that $s !=t$. If the principals of the records do not match that of the SETCLIENTID_CONFIRM, the server returns NFS4ERR_CLID_INUSE, and no relevant leased client state is removed and no recorded callback and callback_ident information for client $\{\mathrm{x}\}$ is changed. Otherwise, the confirmed $\{v, x, c, l, t\}$ record is removed and the unconfirmed $\{\mathrm{V}, \mathrm{x}, \mathrm{C}, \mathrm{k}, \mathrm{s}\}$ is marked as confirmed, thereby modifying recorded and confirmed callback and callback_ident information for client $\{x\}$.

The server does not remove any relevant leased client state.

The server returns NFS4_OK.

- The server has not recorded an unconfirmed $\{\mathrm{V}, \mathrm{x}, \mathrm{c}$, *, * $\}$ and has recorded a confirmed $\{\mathrm{V}, \mathrm{x}, \mathrm{C}, *, \mathrm{~s}\}$. If the principals of the record and of SETCLIENTID_CONFIRM do not match, the server returns NFS4ERR_CLID_INUSE without removing any relevant leased client state and without changing recorded callback and callback_ident values for client $\{\mathrm{x}\}$.

If the principals match, then what has likely happened is that the client never got the response from the SETCLIENTID_CONFIRM, and the DRC entry has been purged. Whatever the scenario, since the principals match, as well as $\{\mathrm{C}, \mathrm{s}\}$ matching a confirmed record, the server leaves client $x^{\prime}$ s relevant leased client state intact, leaves its callback and callback_ident values unmodified, and returns NFS4_OK.

- The server has not recorded a confirmed $\{*, *, c, *, *\}$, and has recorded an unconfirmed $\{\star, x, c, k, s\}$. Even if this is a retry from client, nonetheless the client's first

SETCLIENTID_CONFIRM attempt was not received by the server. Retry or not, the server doesn't know, but it processes it as if were a first try. If the principal of the unconfirmed $\{*, \mathrm{x}, \mathrm{c}, \mathrm{k}, \mathrm{s}\}$ record mismatches that of the SETCLIENTID_CONFIRM request the server returns NFS4ERR_CLID_INUSE without removing any relevant leased client state. 
Otherwise, the server records a confirmed $\{*, \mathrm{x}, \mathrm{c}, \mathrm{k}, \mathrm{s}\}$. If there is also a confirmed $\{*, x, d, *, t\}$, the server MUST remove the client $\mathrm{x}^{\prime} \mathrm{s}$ relevant leased client state, and overwrite the callback state with $\mathrm{k}$. The confirmed record $\{\star, x, d, *, t\}$ is removed.

Server returns NFS4_OK.

- The server has no record of a confirmed or unconfirmed $\{$ * *, C, *, s \}. The server returns NFS4ERR_STALE_CLIENTID. The server does not remove any relevant leased client state, nor does it modify any recorded callback and callback_ident information for any client.

The server needs to cache unconfirmed $\{\mathrm{V}, \mathrm{x}, \mathrm{c}, \mathrm{k}, \mathrm{s}\}$ client records and await for some time their confirmation. As should be clear from the record processing discussions for SETCLIENTID and SETCLIENTID_CONFIRM, there are cases where the server does not deterministically remove unconfirmed client records. To avoid running out of resources, the server is not required to hold unconfirmed records indefinitely. One strategy the server might use is to set a limit on how many unconfirmed client records it will maintain, and then when the limit would be exceeded, remove the oldest record. Another strategy might be to remove an unconfirmed record when some amount of time has elapsed. The choice of the amount of time is fairly arbitrary but it is surely no higher than the server's lease time period. Consider that leases need to be renewed before the lease time expires via an operation from the client. If the client cannot issue a SETCLIENTID_CONFIRM after a SETCLIENTID before a period of time equal to that of a lease expires, then the client is unlikely to be able maintain state on the server during steady state operation.

If the client does send a SETCLIENTID_CONFIRM for an unconfirmed record that the server has already deleted, the client will get NFS4ERR_STALE_CLIENTID back. If so, the client should then start over, and send SETCLIENTID to reestablish an unconfirmed client record and get back an unconfirmed clientid and setclientid_confirm verifier. The client should then send the SETCLIENTID_CONFIRM to confirm the clientid.

SETCLIENTID_CONFIRM does not establish or renew a lease. However, if SETCLIENTID_CONFIRM removes relevant leased client state, and that state does not include existing delegations, the server MUST allow the client a period of time no less than the value of lease_time attribute, to reclaim, (via the CLAIM_DELEGATE_PREV claim type of the OPEN operation) its delegations before removing unreclaimed delegations. 


\section{ERRORS}

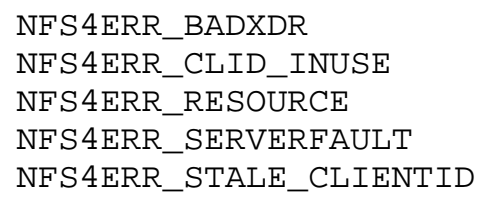

The VERIFY operation is used to verify that attributes have a value assumed by the client before proceeding with following operations in the compound request. If any of the attributes do not match then the error NFS4ERR_NOT_SAME must be returned. The current filehandle retains its value after successful completion of the operation.

IMP LEMENTATION

One possible use of the VERIFY operation is the following compound sequence. With this the client is attempting to verify that the file being removed will match what the client expects to be removed. This sequence can help prevent the unintended deletion of a file.

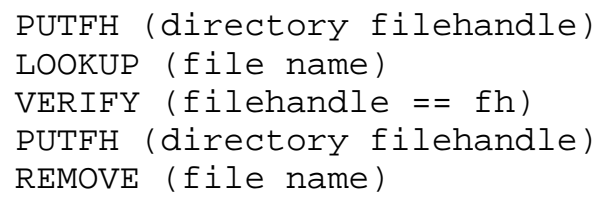


This sequence does not prevent a second client from removing and creating a new file in the middle of this sequence but it does help avoid the unintended result.

In the case that a recommended attribute is specified in the VERIFY operation and the server does not support that attribute for the filesystem object, the error NFS4ERR_ATTRNOTSUPP is returned to the client.

When the attribute rdattr_error or any write-only attribute (e.g., time_modify_set) is specified, the error NFS4ERR_INVAL is returned to the client.

\section{ERRORS}

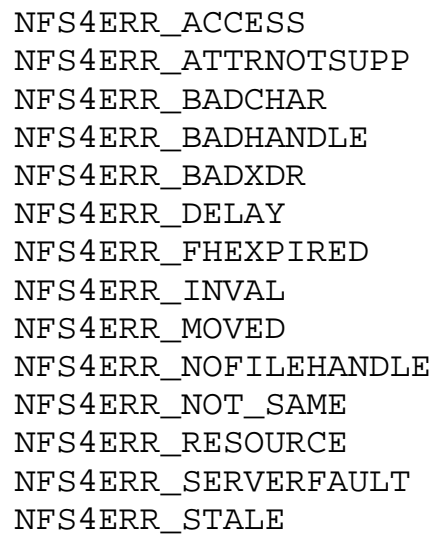




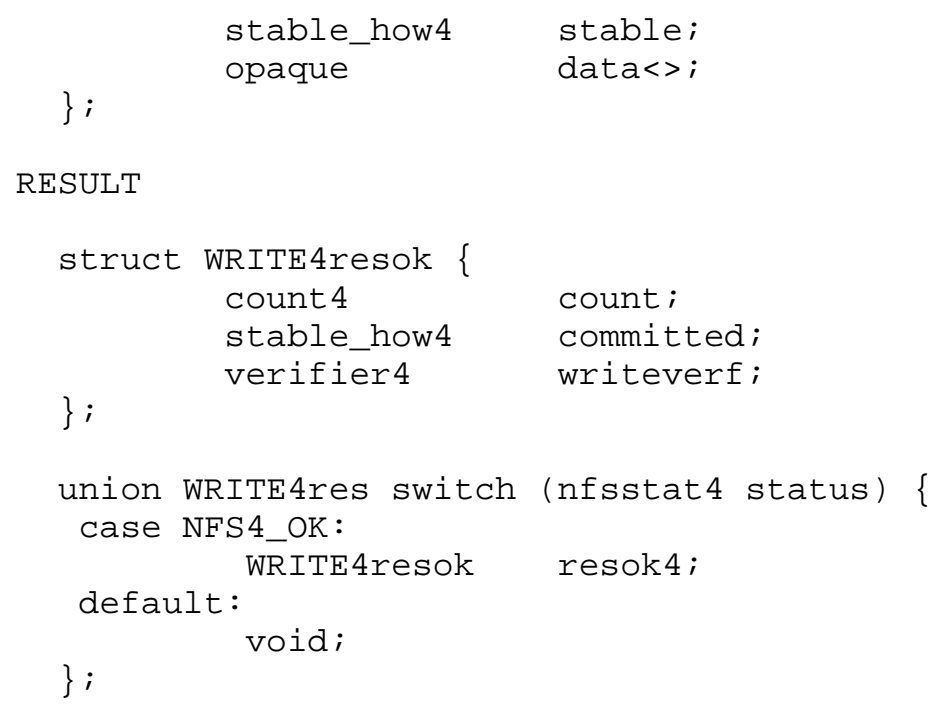

DESCRIPTION

The WRITE operation is used to write data to a regular file. The target file is specified by the current filehandle. The offset specifies the offset where the data should be written. An offset of 0 (zero) specifies that the write should start at the beginning of the file. The count, as encoded as part of the opaque data parameter, represents the number of bytes of data that are to be written. If the count is 0 (zero), the WRITE will succeed and return a count of 0 (zero) subject to permissions checking. The server may choose to write fewer bytes than requested by the client.

Part of the write request is a specification of how the write is to be performed. The client specifies with the stable parameter the method of how the data is to be processed by the server. If stable is FILE_SYNC4, the server must commit the data written plus all filesystem metadata to stable storage before returning results. This corresponds to the NFS version 2 protocol semantics. Any other behavior constitutes a protocol violation. If stable is DATA_SYNC4, then the server must commit all of the data to stable storage and enough of the metadata to retrieve the data before returning. The server implementor is free to implement DATA_SYNC4 in the same fashion as FILE_SYNC4, but with a possible performance drop. If stable is UNSTABLE4, the server is free to commit any part of the data and the metadata to stable storage, including all or none, before returning a reply to the client. There is no guarantee whether or when any uncommitted data will subsequently be committed to stable storage. The only guarantees made by the server are that it will not 
destroy any data without changing the value of verf and that it will not commit the data and metadata at a level less than that requested by the client.

The stateid value for a WRITE request represents a value returned from a previous record lock or share reservation request. The stateid is used by the server to verify that the associated share reservation and any record locks are still valid and to update lease timeouts for the client.

Upon successful completion, the following results are returned. The count result is the number of bytes of data written to the file. The server may write fewer bytes than requested. If so, the actual number of bytes written starting at location, offset, is returned.

The server also returns an indication of the level of commitment of the data and metadata via committed. If the server committed all data and metadata to stable storage, committed should be set to FILE_SYNC4. If the level of commitment was at least as strong as DATA_SYNC4, then committed should be set to DATA_SYNC4. Otherwise, committed must be returned as UNSTABLE4. If stable was FILE4_SYNC, then committed must also be FILE_SYNC4: anything else constitutes a protocol violation. If stable was DATA_SYNC4, then committed may be FILE_SYNC4 or DATA_SYNC4: anything else constitutes a protocol violation. If stable was UNSTABLE4, then committed may be either FILE_SYNC4, DATA_SYNC4, or UNSTABLE4.

The final portion of the result is the write verifier. The write verifier is a cookie that the client can use to determine whether the server has changed instance (boot) state between a call to WRITE and a subsequent call to either WRITE or COMMIT. This cookie must be consistent during a single instance of the NFS version 4 protocol service and must be unique between instances of the NFS version 4 protocol server, where uncommitted data may be lost.

If a client writes data to the server with the stable argument set to UNSTABLE4 and the reply yields a committed response of DATA_SYNC4 or UNSTABLE4, the client will follow up some time in the future with a COMMIT operation to synchronize outstanding asynchronous data and metadata with the server's stable storage, barring client error. It is possible that due to client crash or other error that a subsequent COMMIT will not be received by the server.

For a WRITE with a stateid value of all bits 0 , the server MAY allow the WRITE to be serviced subject to mandatory file locks or the current share deny modes for the file. For a WRITE with a stateid 
value of all bits 1, the server MUST NOT allow the WRITE operation to bypass locking checks at the server and are treated exactly the same as if a stateid of all bits 0 were used.

On success, the current filehandle retains its value.

\section{IMP LEMENTATION}

It is possible for the server to write fewer bytes of data than requested by the client. In this case, the server should not return an error unless no data was written at all. If the server writes less than the number of bytes specified, the client should issue another WRITE to write the remaining data.

It is assumed that the act of writing data to a file will cause the time_modified of the file to be updated. However, the time_modified of the file should not be changed unless the contents of the file are changed. Thus, a WRITE request with count set to 0 should not cause the time_modified of the file to be updated.

The definition of stable storage has been historically a point of contention. The following expected properties of stable storage may help in resolving design issues in the implementation. Stable storage is persistent storage that survives:

1. Repeated power failures.

2. Hardware failures (of any board, power supply, etc.).

3. Repeated software crashes, including reboot cycle.

This definition does not address failure of the stable storage module itself.

The verifier is defined to allow a client to detect different instances of an NFS version 4 protocol server over which cached, uncommitted data may be lost. In the most likely case, the verifier allows the client to detect server reboots. This information is required so that the client can safely determine whether the server could have lost cached data. If the server fails unexpectedly and the client has uncommitted data from previous WRITE requests (done with the stable argument set to UNSTABLE4 and in which the result committed was returned as UNSTABLE4 as well) it may not have flushed cached data to stable storage. The burden of recovery is on the client and the client will need to retransmit the data to the server.

A suggested verifier would be to use the time that the server was booted or the time the server was last started (if restarting the server without a reboot results in lost buffers). 
The committed field in the results allows the client to do more effective caching. If the server is committing all WRITE requests to stable storage, then it should return with committed set to FILE_SYNC4, regardless of the value of the stable field in the arguments. A server that uses an NVRAM accelerator may choose to implement this policy. The client can use this to increase the effectiveness of the cache by discarding cached data that has already been committed on the server.

Some implementations may return NFS4ERR_NOSPC instead of NFS4ERR_DQUOT when a user's quota is exceeded. In the case that the current filehandle is a directory, the server will return NFS4ERR_ISDIR. If the current filehandle is not a regular file or a directory, the server will return NFS4ERR_INVAL.

If mandatory file locking is on for the file, and corresponding record of the data to be written file is read or write locked by an owner that is not associated with the stateid, the server will return NFS4ERR_LOCKED. If so, the client must check if the owner corresponding to the stateid used with the WRITE operation has a conflicting read lock that overlaps with the region that was to be written. If the stateid's owner has no conflicting read lock, then the client should try to get the appropriate write record lock via the LOCK operation before re-attempting the WRITE. When the WRITE completes, the client should release the record lock via LOCKU.

If the stateid's owner had a conflicting read lock, then the client has no choice but to return an error to the application that attempted the WRITE. The reason is that since the stateid's owner had a read lock, the server either attempted to temporarily effectively upgrade this read lock to a write lock, or the server has no upgrade capability. If the server attempted to upgrade the read lock and failed, it is pointless for the client to re-attempt the upgrade via the LOCK operation, because there might be another client also trying to upgrade. If two clients are blocked trying upgrade the same lock, the clients deadlock. If the server has no upgrade capability, then it is pointless to try a LOCK operation to upgrade.

\section{ERRORS}

NFS 4ERR_ACCESS

NFS 4ERR_ADMIN_REVOKED

NF S 4ERR_BADHANDLE

NFS 4ERR_BAD_STATEID

NFS 4ERR_BADXDR

NFS 4ERR_DELAY

NFS 4ERR_DQUOT

NFS 4ERR_EXPIRED 


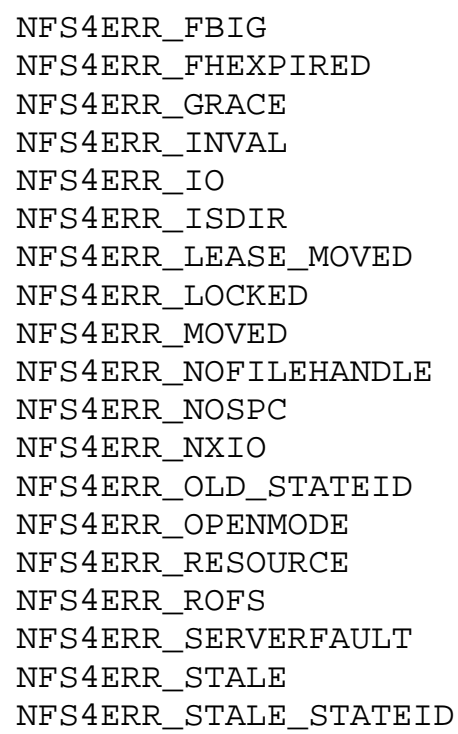

14.2.37. Operation 39: RELEASE_LOCKOWNER - Release Lockowner State SYNOPSIS

lockowner $\rightarrow()$

ARGUMENT

struct RELEASE_LOCKOWNER4args \{

\} ; lock_owner4 lock_owner;

RESULT

struct RELEASE_LOCKOWNER4res \{ nfsstat 4 status;

\} ;

DESCRIPTION

This operation is used to notify the server that the lock_owner is no longer in use by the client. This allows the server to release cached state related to the specified lock_owner. If file locks, associated with the lock_owner, are held at the server, the error NFS4ERR_LOCKS_HELD will be returned and no further action will be taken. 


\section{IMP LEMENTATION}

The client may choose to use this operation to ease the amount of server state that is held. Depending on behavior of applications at the client, it may be important for the client to use this operation since the server has certain obligations with respect to holding a reference to a lock_owner as long as the associated file is open. Therefore, if the client knows for certain that the lock_owner will no longer be used under the context of the associated open_owner4, it should use RELEASE_LOCKOWNER.

\section{ERRORS}

NFS 4ERR_ADMIN_REVOKED
NFS 4ERR_BADXDR
NFS 4ERR_EXP IRED
NFS 4ERR_LEASE_MOVED
NFS 4 ERR_LOCKS_HELD
NFS 4ERR_RESOURCE
NFS 4ERR_SERVERFAULT
NFS 4ERR_STALE_CLIENT ID

14.2.38. Operation 10044: ILLEGAL - Illegal operation SYNOPSIS $<$ null > -> () ARGUMENT void; RESULT

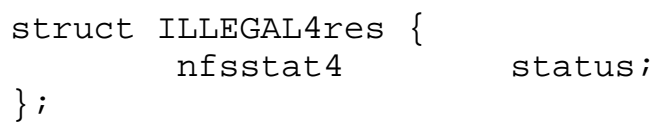


IMP LEMENTATION

A client will probably not send an operation with code OP_ILLEGAL but if it does, the response will be ILLEGAL4res just as it would be with any other invalid operation code. Note that if the server gets an illegal operation code that is not OP_ILLEGAL, and if the server checks for legal operation codes during the XDR decode phase, then the ILLEGAL4res would not be returned.

ERRORS

NFS 4ERR_OP_ILLEGAL

15. NFS version 4 Callback Procedures

The procedures used for callbacks are defined in the following sections. In the interest of clarity, the terms "client" and "server" refer to NFS clients and servers, despite the fact that for an individual callback RPC, the sense of these terms would be precisely the opposite.

15.1. Procedure 0: CB_NULL - No Operation

SYNOPSIS

$<$ null $>$

ARGUMENT

void;

RESULT

void;

DESCRIPTION

Standard NULL procedure. Void argument, void response. Even though there is no direct functionality associated with this procedure, the server will use CB_NULL to confirm the existence of a path for RPCs from server to client.

ERRORS

None. 


\subsection{Procedure 1: CB_COMPOUND - Compound Operations}

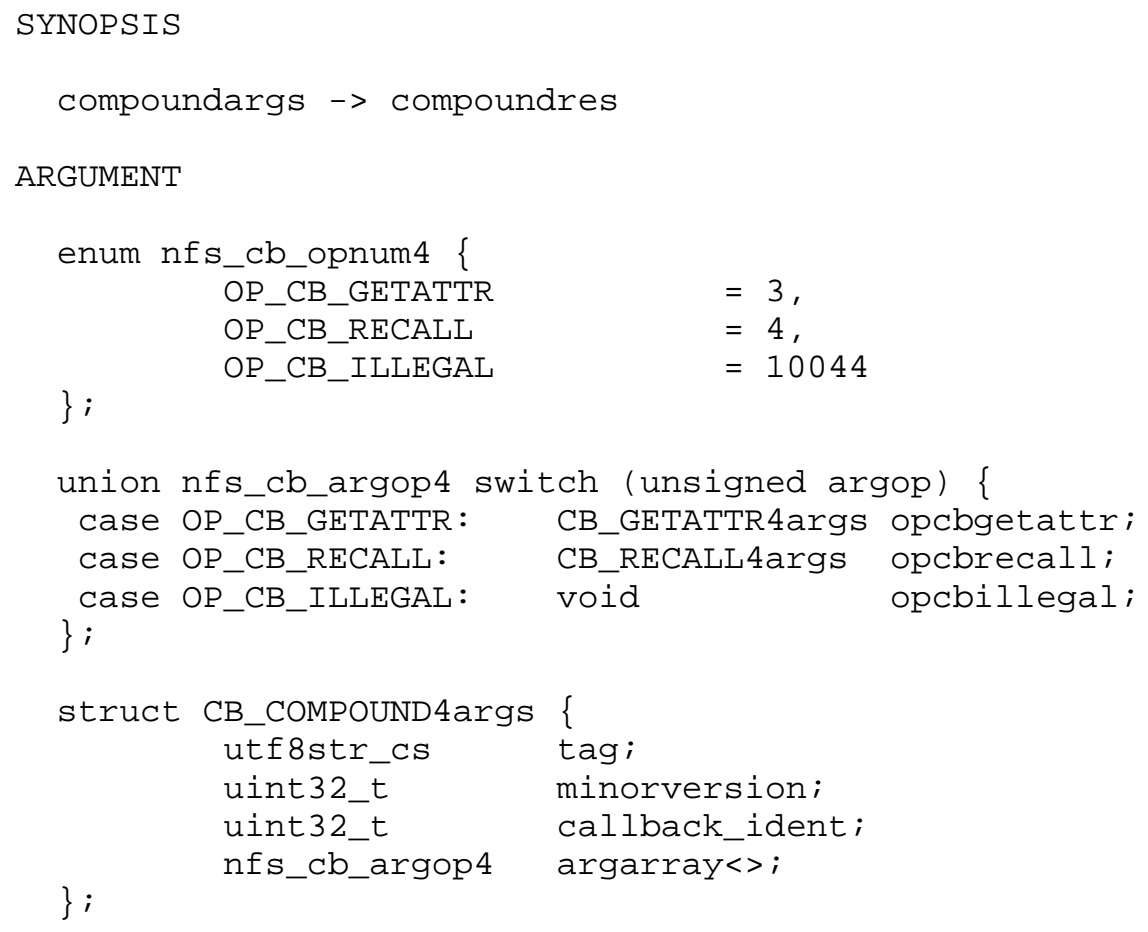


In the processing of the CB_COMPOUND procedure, the client may find that it does not have the available resources to execute any or all of the operations within the CB_COMPOUND sequence. In this case, the error NFS4ERR_RESOURCE will be returned for the particular operation within the CB_COMPOUND procedure where the resource exhaustion occurred. This assumes that all previous operations within the CB_COMPOUND sequence have been evaluated successfully.

Contained within the CB_COMPOUND results is a 'status' field. This status must be equivalent to the status of the last operation that was executed within the CB_COMPOUND procedure. Therefore, if an operation incurred an error then the 'status' value will be the same error value as is being returned for the operation that failed.

For the definition of the "tag" field, see the section "Procedure 1: COMPOUND - Compound Operations".

The value of callback_ident is supplied by the client during SETCLIENTID. The server must use the client supplied callback_ident during the CB_COMPOUND to allow the client to properly identify the server.

Illegal operation codes are handled in the same way as they are handled for the COMPOUND procedure.

\section{IMP LEMENTATION}

The CB_COMPOUND procedure is used to combine individual operations into a single RPC request. The client interprets each of the operations in turn. If an operation is executed by the client and the status of that operation is NFS4_OK, then the next operation in the CB_COMPOUND procedure is executed. The client continues this process until there are no more operations to be executed or one of the operations has a status value other than NFS4_OK.

\section{ERRORS}

NFS 4ERR_BADHANDLE
NF S 4ERR_BAD_STATEID
NF S 4ERR_BADXDR
NFS 4ERR_OP_ILLEGAL
NFS 4ERR_RESOURCE
NFS 4ERR_SERVERFAULT


15.2.1. Operation 3: CB_GETATTR - Get Attributes

SYNOPSIS

fh, attr_request $->$ attrmask, attr_vals

ARGUMENT

struct CB_GETATTR4args \{

nfs_fh4 fh;

\} ;

bitmap4 attr_request;

RESULT

struct CB_GETATTR4resok \{

\} ;

fattr4 obj_attributes;

union CB_GETATTR4res switch (nfsstat4 status) \{ case NFS4_OK:

default:

CB_GETATTR4resok resok4;

\} ;

void;

DESCRIPTION

The CB_GETATTR operation is used by the server to obtain the current modified state of a file that has been write delegated. The attributes size and change are the only ones guaranteed to be serviced by the client. See the section "Handling of CB_GETATTR" for a full description of how the client and server are to interact with the use of CB_GETATTR.

If the filehandle specified is not one for which the client holds a write open delegation, an NFS4ERR_BADHANDLE error is returned.

IMP LEMENTATION

The client returns attrmask bits and the associated attribute values only for the change attribute, and attributes that it may change (time_modify, and size). 


\section{ERRORS}

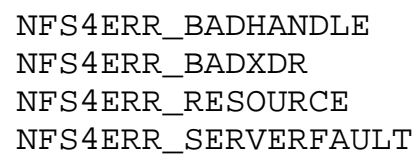


IMP LEMENTATION

The client should reply to the callback immediately. Replying does not complete the recall except when an error was returned. The recall is not complete until the delegation is returned using a DELEGRETURN .

ERRORS

NFS 4ERR_BADHANDLE
NFS 4ERR_BAD_STATEID
NFS 4ERR_BADXDR
NFS 4ERR_RESOURCE
NFS 4ERR_SERVERFAULT

15.2.3. Operation 10044: CB_ILLEGAL - Illegal Callback Operation SYNOPSIS

$<\operatorname{null}>->()$

ARGUMENT

void;

RESULT

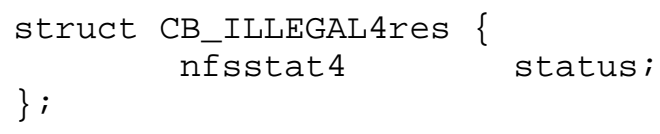

A server will probably not send an operation with code OP_CB_ILLEGAL but if it does, the response will be CB_ILLEGAL4res just as it would be with any other invalid operation code. Note that if the client 
gets an illegal operation code that is not OP_ILLEGAL, and if the client checks for legal operation codes during the XDR decode phase, then the CB_ILLEGAL4res would not be returned.

\section{ERRORS}

NFS 4ERR_OP_ILLEGAL

\section{Security Considerations}

NFS has historically used a model where, from an authentication perspective, the client was the entire machine, or at least the source IP address of the machine. The NFS server relied on the NFS client to make the proper authentication of the end-user. The NFS server in turn shared its files only to specific clients, as identified by the client's source IP address. Given this model, the AUTH_SYS RPC security flavor simply identified the end-user using the client to the NFS server. When processing NFS responses, the client ensured that the responses came from the same IP address and port number that the request was sent to. While such a model is easy to implement and simple to deploy and use, it is certainly not a safe model. Thus, NFSv4 mandates that implementations support a security model that uses end to end authentication, where an end-user on a client mutually authenticates (via cryptographic schemes that do not expose passwords or keys in the clear on the network) to a principal on an NFS server. Consideration should also be given to the integrity and privacy of NFS requests and responses. The issues of end to end mutual authentication, integrity, and privacy are discussed as part of the section on "RPC and Security Flavor".

Note that while NFSv4 mandates an end to end mutual authentication model, the "classic" model of machine authentication via IP address checking and AUTH_SYS identification can still be supported with the caveat that the AUTH_SYS flavor is neither MANDATORY nor RECOMMENDED by this specification, and so interoperability via AUTH_SYS is not assured.

For reasons of reduced administration overhead, better performance and/or reduction of CPU utilization, users of NFS version 4 implementations may choose to not use security mechanisms that enable integrity protection on each remote procedure call and response. The use of mechanisms without integrity leaves the customer vulnerable to an attacker in between the NFS client and server that modifies the $\mathrm{RPC}$ request and/or the response. While implementations are free to provide the option to use weaker security mechanisms, there are two operations in particular that warrant the implementation overriding user choices. 
The first such operation is SECINFO. It is recommended that the client issue the SECINFO call such that it is protected with a security flavor that has integrity protection, such as RPCSEC_GSS with a security triple that uses either rpc_gss_svc_integrity or rpc_gss_svc_privacy (rpc_gss_svc_privacy includes integrity protection) service. Without integrity protection encapsulating SECINFO and therefore its results, an attacker in the middle could modify results such that the client might select a weaker algorithm in the set allowed by server, making the client and/or server vulnerable to further attacks.

The second operation that should definitely use integrity protection is any GETATTR for the fs_locations attribute. The attack has two steps. First the attacker modifies the unprotected results of some operation to return NFS4ERR_MOVED. Second, when the client follows up with a GETATTR for the fs_locations attribute, the attacker modifies the results to cause the client migrate its traffic to a server controlled by the attacker.

Because the operations SETCLIENTID/SETCLIENTID_CONFIRM are responsible for the release of client state, it is imperative that the principal used for these operations is checked against and match the previous use of these operations. See the section "Client ID" for further discussion.

\section{IANA Considerations}

\subsection{Named Attribute Definition}

The NFS version 4 protocol provides for the association of named attributes to files. The name space identifiers for these attributes are defined as string names. The protocol does not define the specific assignment of the name space for these file attributes. Even though the name space is not specifically controlled to prevent collisions, an IANA registry has been created for the registration of NFS version 4 named attributes. Registration will be achieved through the publication of an Informational RFC and will require not only the name of the attribute but the syntax and semantics of the named attribute contents; the intent is to promote interoperability where common interests exist. While application developers are allowed to define and use attributes as needed, they are encouraged to register the attributes with IANA.

\subsection{ONC RPC Network Identifiers (netids)}

The section "Structured Data Types" discussed the r_netid field and the corresponding r_addr field of a clientaddr4 structure. The NFS version 4 protocol depends on the syntax and semantics of these 
fields to effectively communicate callback information between client and server. Therefore, an IANA registry has been created to include the values defined in this document and to allow for future expansion based on transport usage/availability. Additions to this ONC RPC Network Identifier registry must be done with the publication of an $\mathrm{RFC}$.

The initial values for this registry are as follows (some of this text is replicated from section 2.2 for clarity):

The Network Identifier (or r_netid for short) is used to specify a transport protocol and associated universal address (or r_addr for short). The syntax of the Network Identifier is a US-ASCII string. The initial definitions for r_netid are:

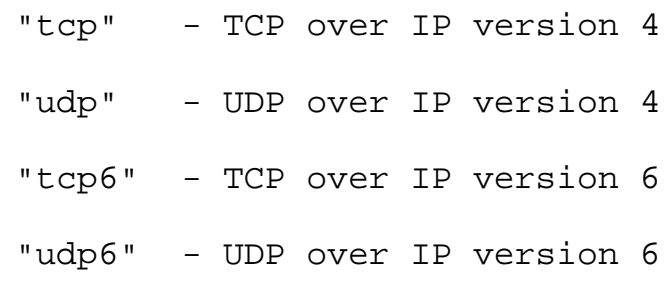


The suffix "p1.p2" is the service port, and is computed the same way as with universal addresses for "tcp" and "udp". The prefix, "x1:x2:x3:x4:x5:x6:x7:x8", is the standard textual form for representing an IPv6 address as defined in section 2.2 of [RFC2373]. Additionally, the two alternative forms specified in section 2.2 of [RFC2373] are also acceptable.

As mentioned, the registration of new Network Identifiers will require the publication of an Information RFC with similar detail as listed above for the Network Identifier itself and corresponding Universal Address.

18. RPC definition file

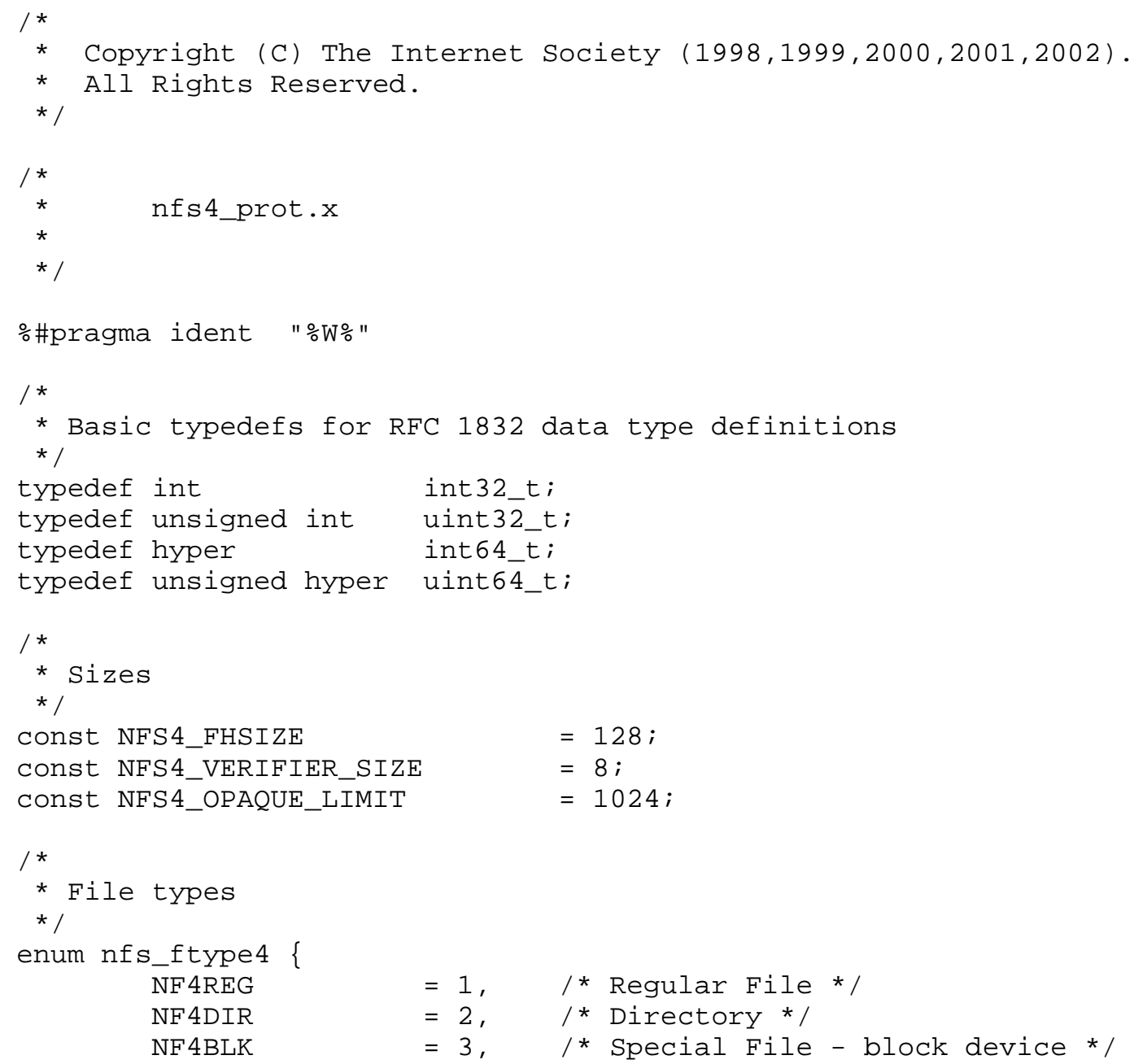




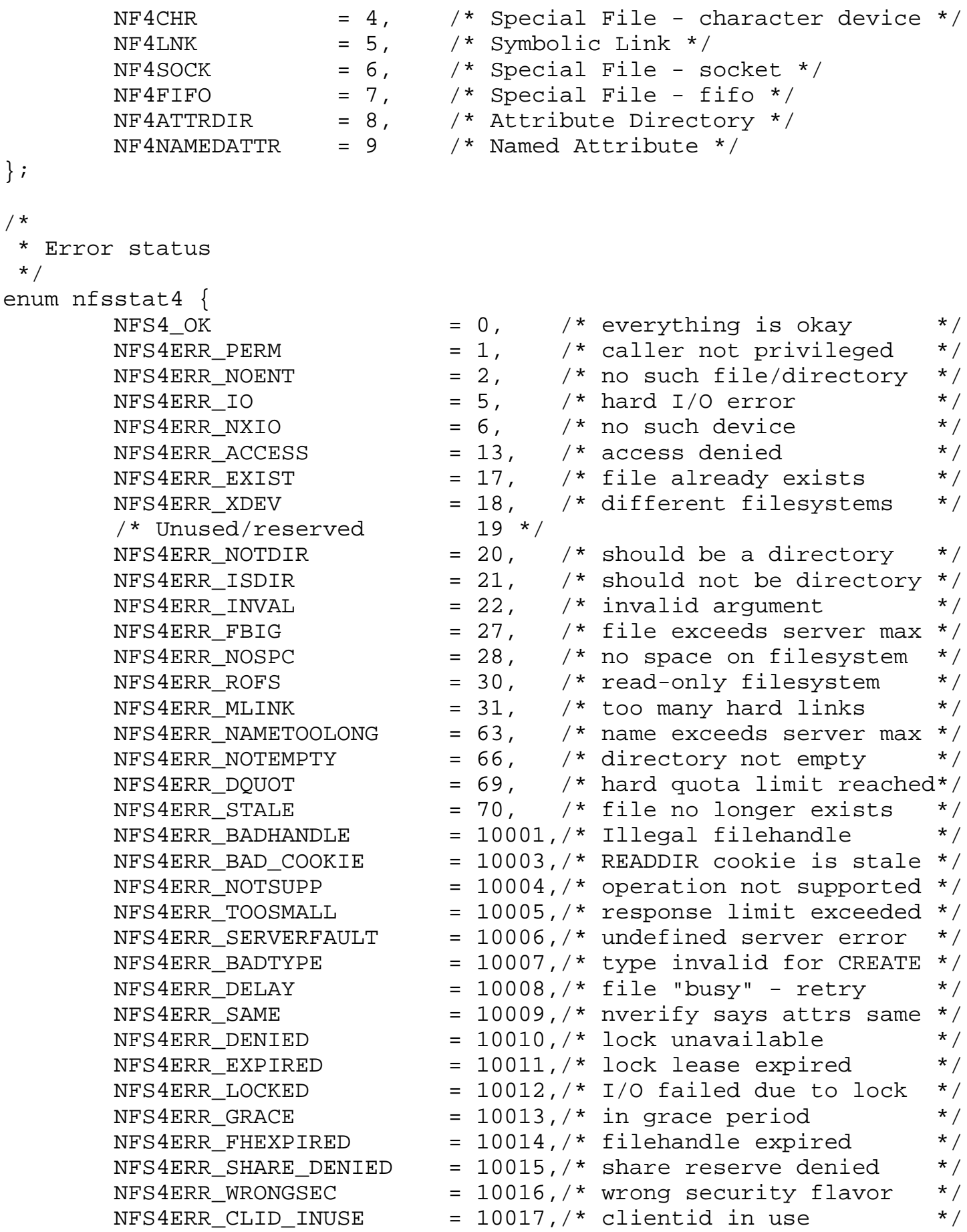




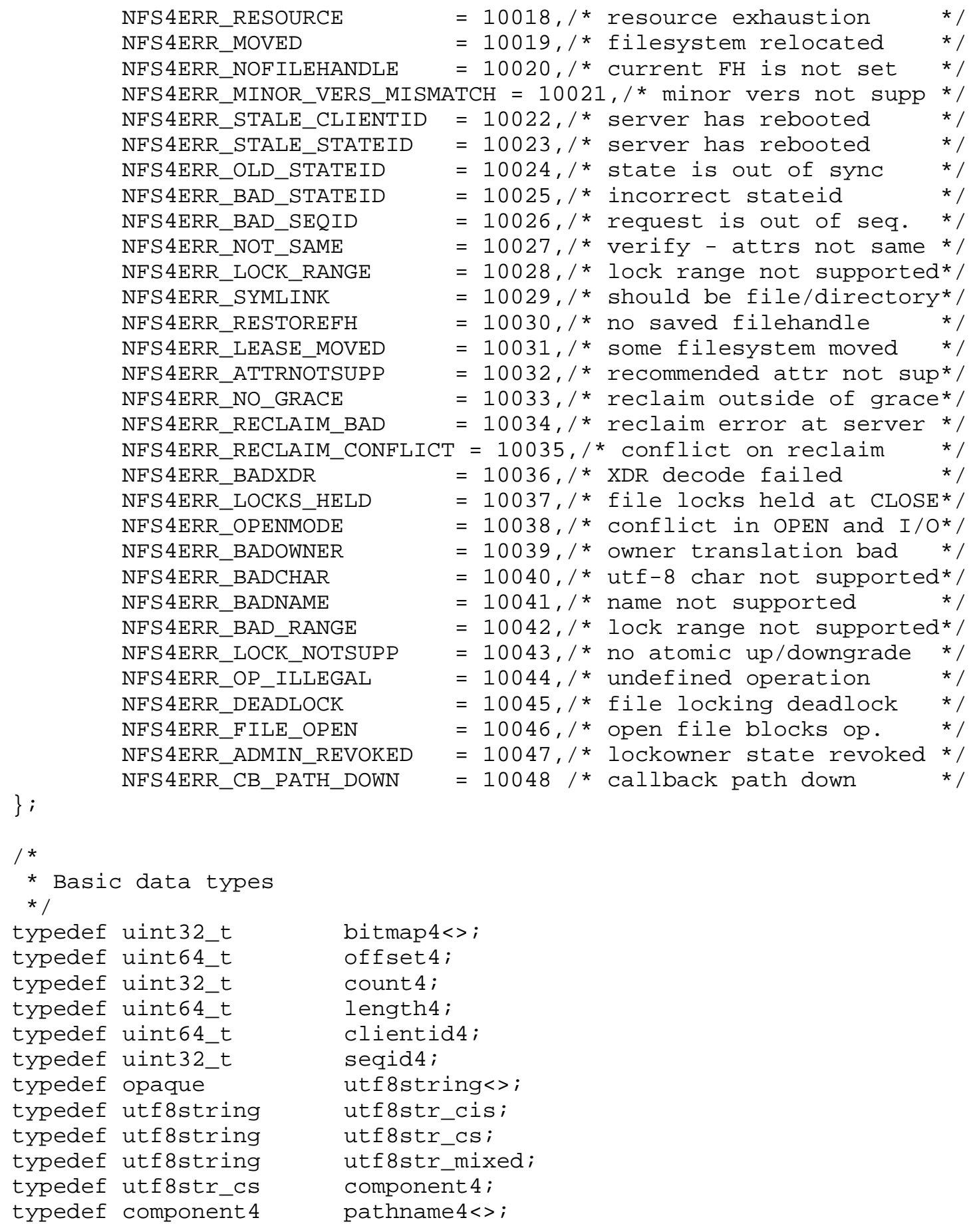




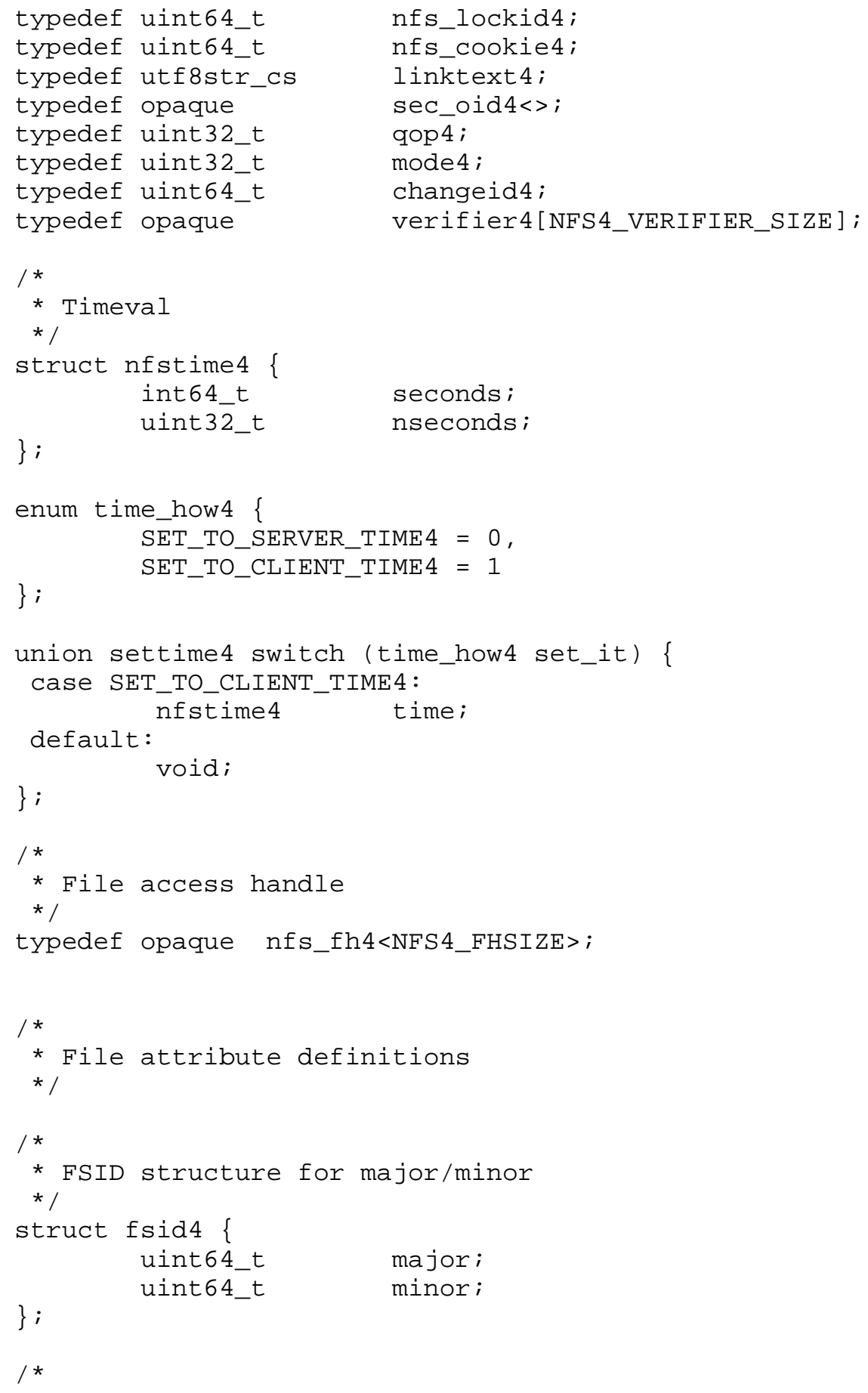




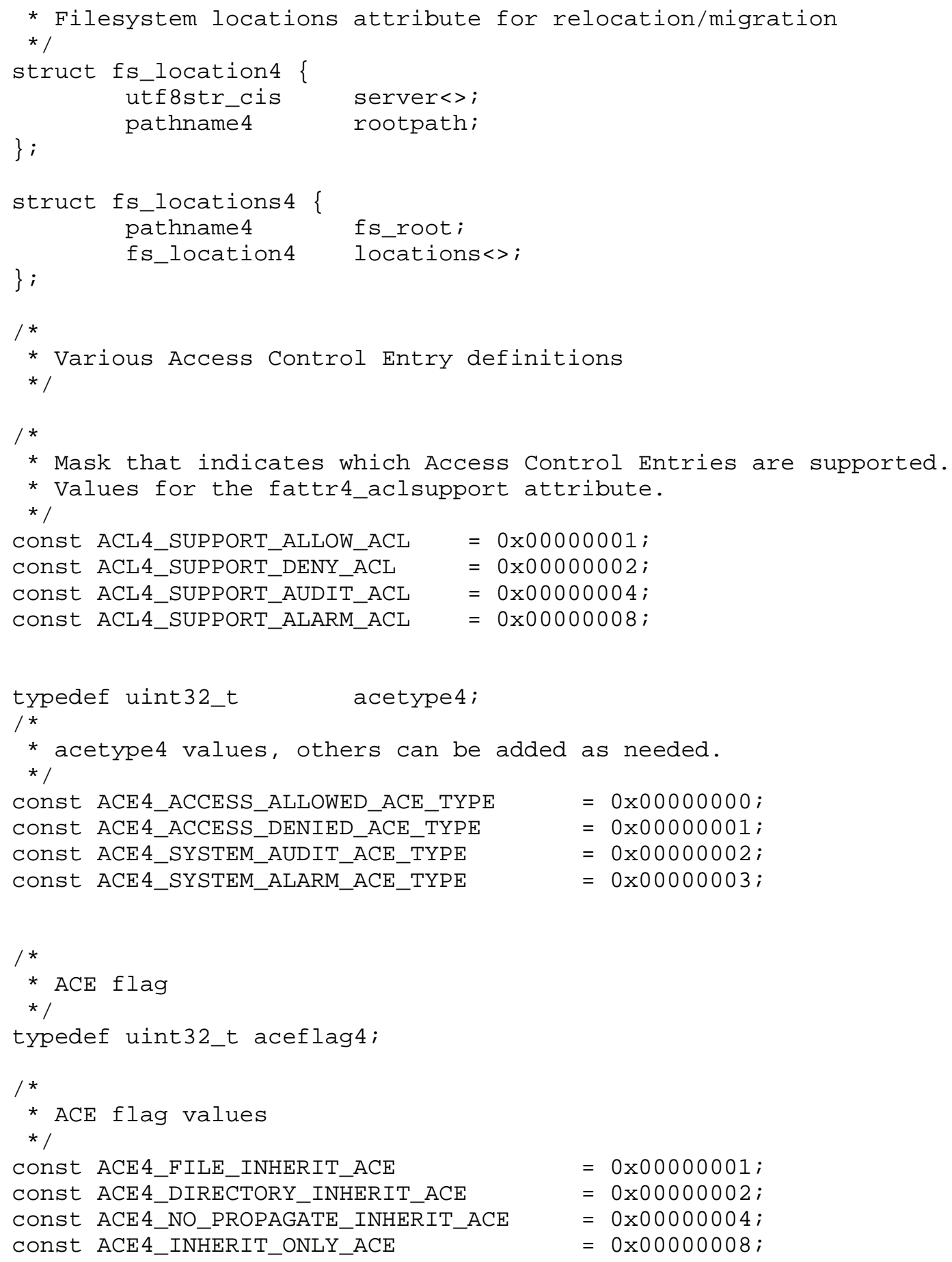




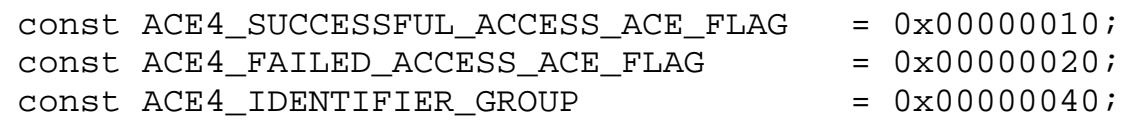




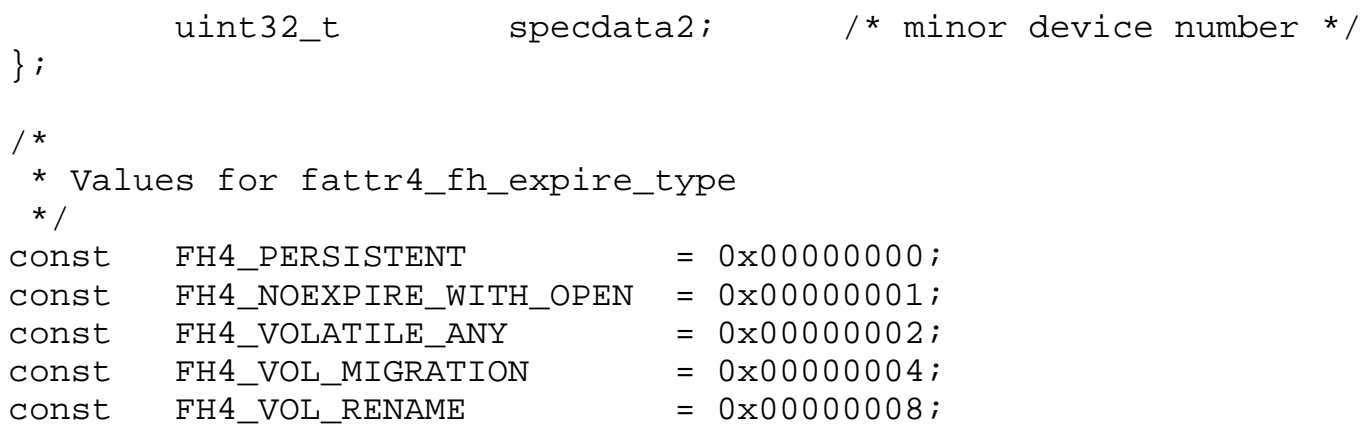

typedef bitmap 4

typedef nfs_ftype 4

typedef uint32_t

typedef changeid4

typedef uint64_t

typedef bool

typedef bool

typedef bool

typedef fsid4

typedef bool

typedef uint32_t

typedef nfsstat 4

typedef nfsace 4

typedef uint32_t

typedef bool

typedef bool

typedef bool

typedef bool

typedef bool

typedef uint 64_t

typedef uint64_t

typedef nfs_fh 4

typedef uint64_t

typedef uint 64_t

typedef fs_locations 4

typedef bool

typedef bool

typedef uint64_t

typedef uint32_t

typedef uint32_t

typedef uint64_t

typedef uint 64_t

typedef utf8str_cs

typedef mode4

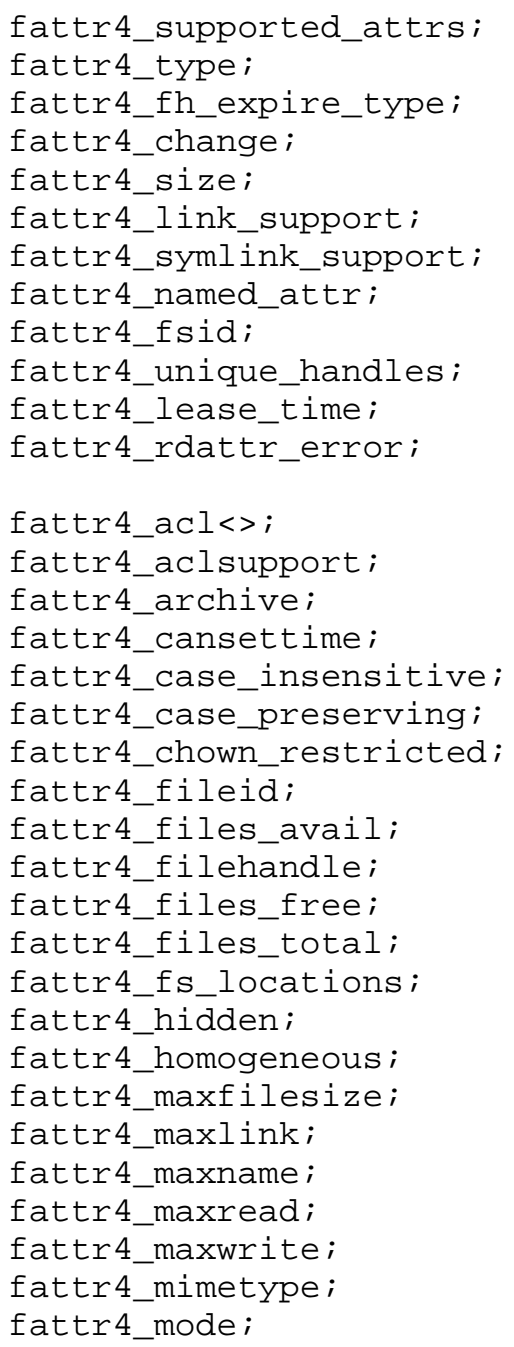

Standards Track

[Page 241] 


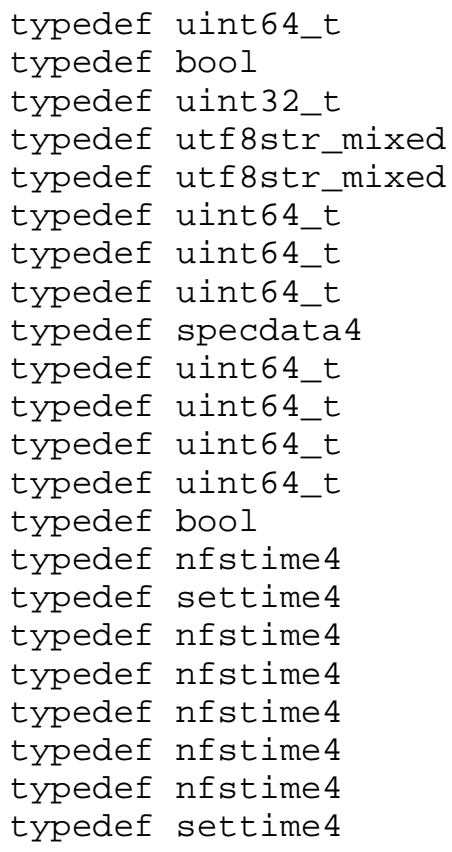

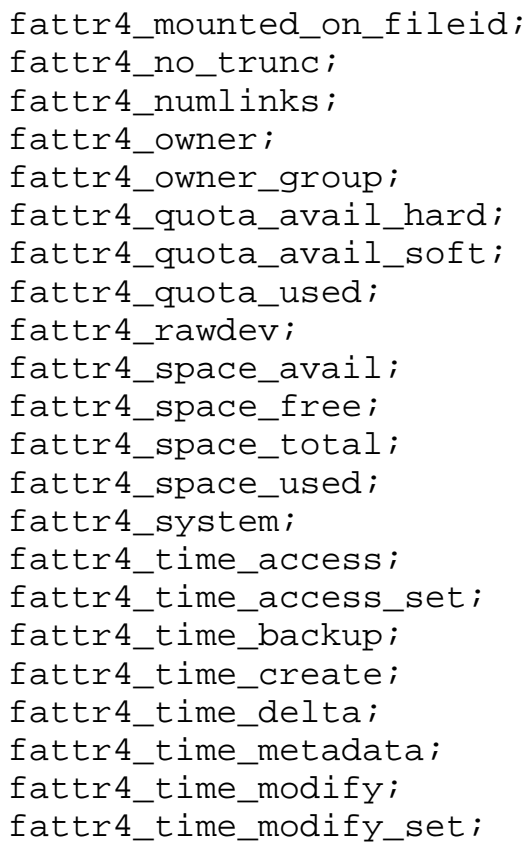

$/ \star$

* Mandatory Attributes

*

const FATTR4_SUPPORTED_ATTRS $=0$;

const FATTR4_TYPE $=1$;

const FATTR4_FH_EXPIRE_TYPE $=2$;

const FATTR4_CHANGE $=3$;

const FATTR4_SIZE $=4$;

const FATTR4_LINK_SUPPORT = 5;

const FATTR4_SYMLINK_SUPPORT $=6$;

const FATTR4_NAMED_ATTR $=7$;

const FATTR4_FSID $=8$;

const FATTR4_UNIQUE_HANDLES $=9$;

const FATTR4_LEASE_TIME $=10$;

const FATTR4_RDATTR_ERROR = 11;

const FATTR4_FILEHANDLE $=19$;

/*

* Recommended Attributes

* /

const FATTR4_ACL = 12;

const FATTR4_ACLSUPPORT = 13;

const FATTR4_ARCHIVE = 14;

const FATTR4_CANSETTIME $=15$; 


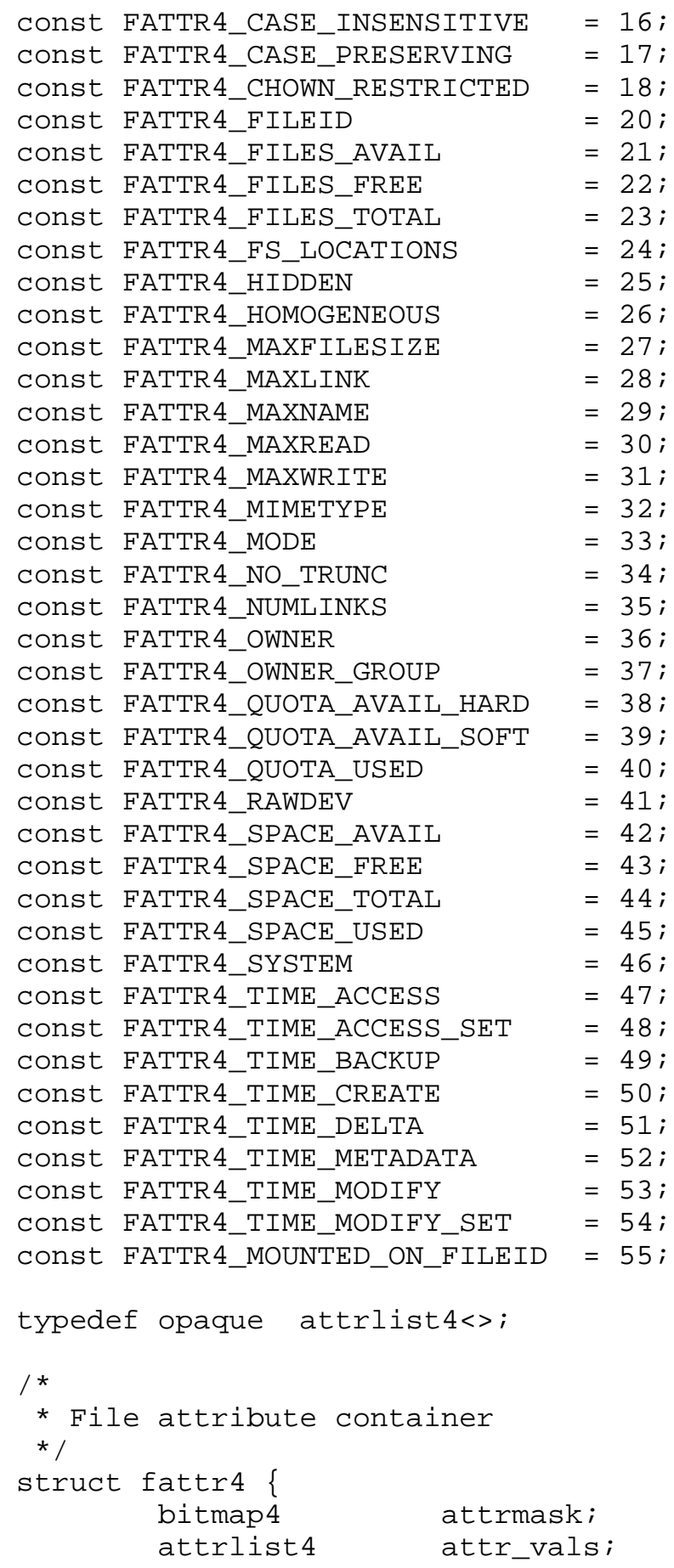


\};

/*

* Change info for the client

* $/$

struct change_info4 bool atomic; changeid4 before;

\} ; changeid4 after;

struct clientaddr4 /* see struct rpcb in RFC 1833 */ 


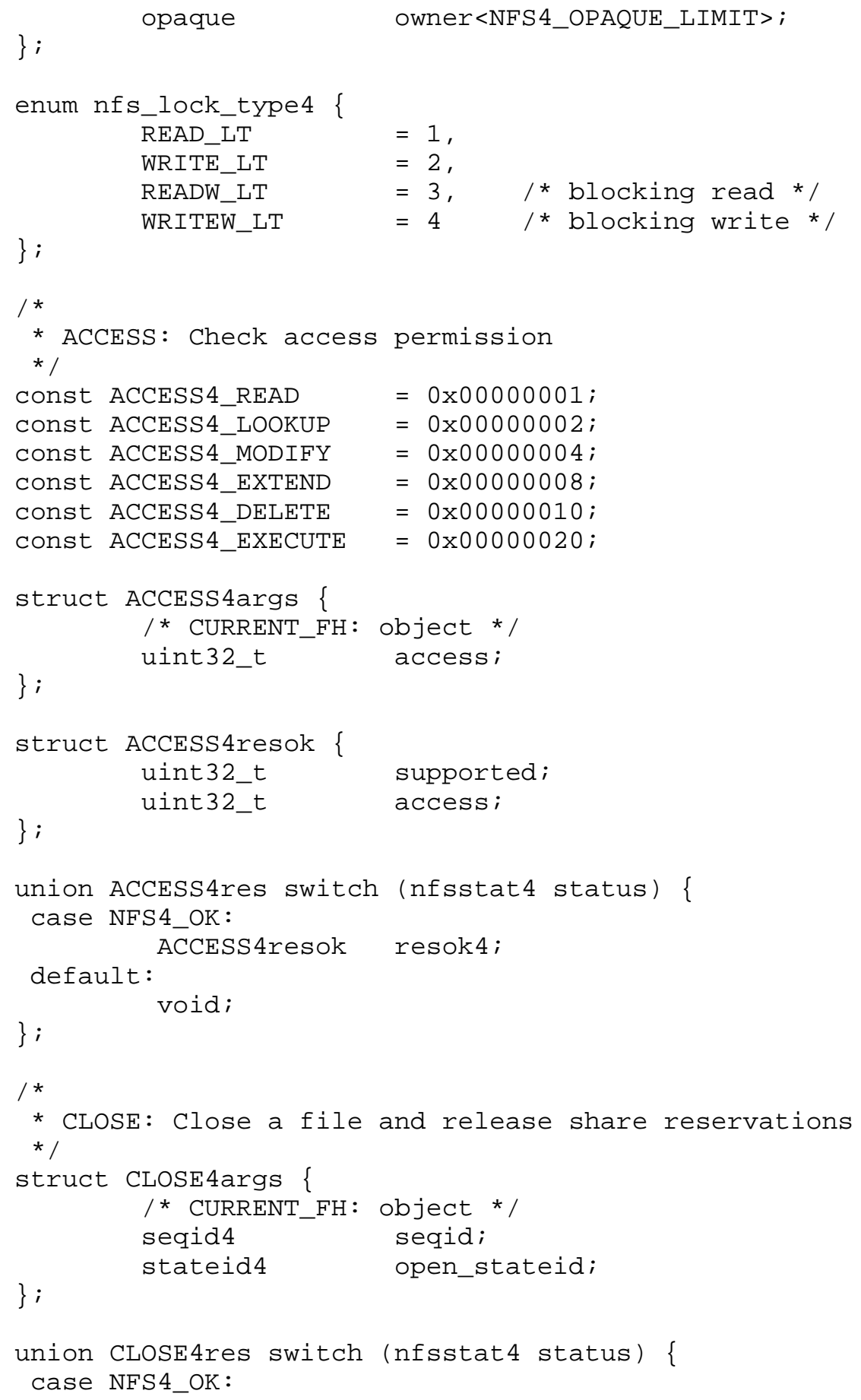




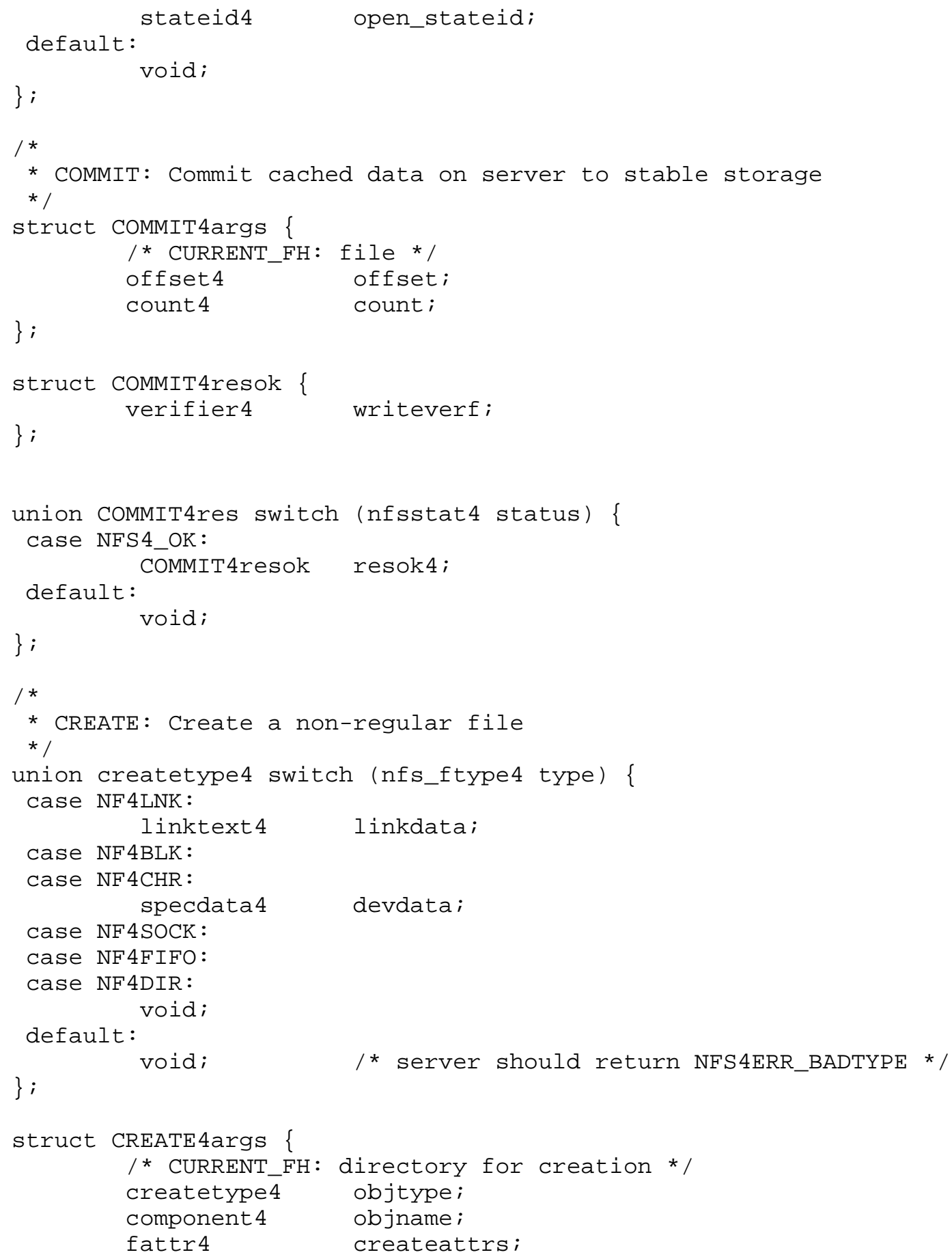




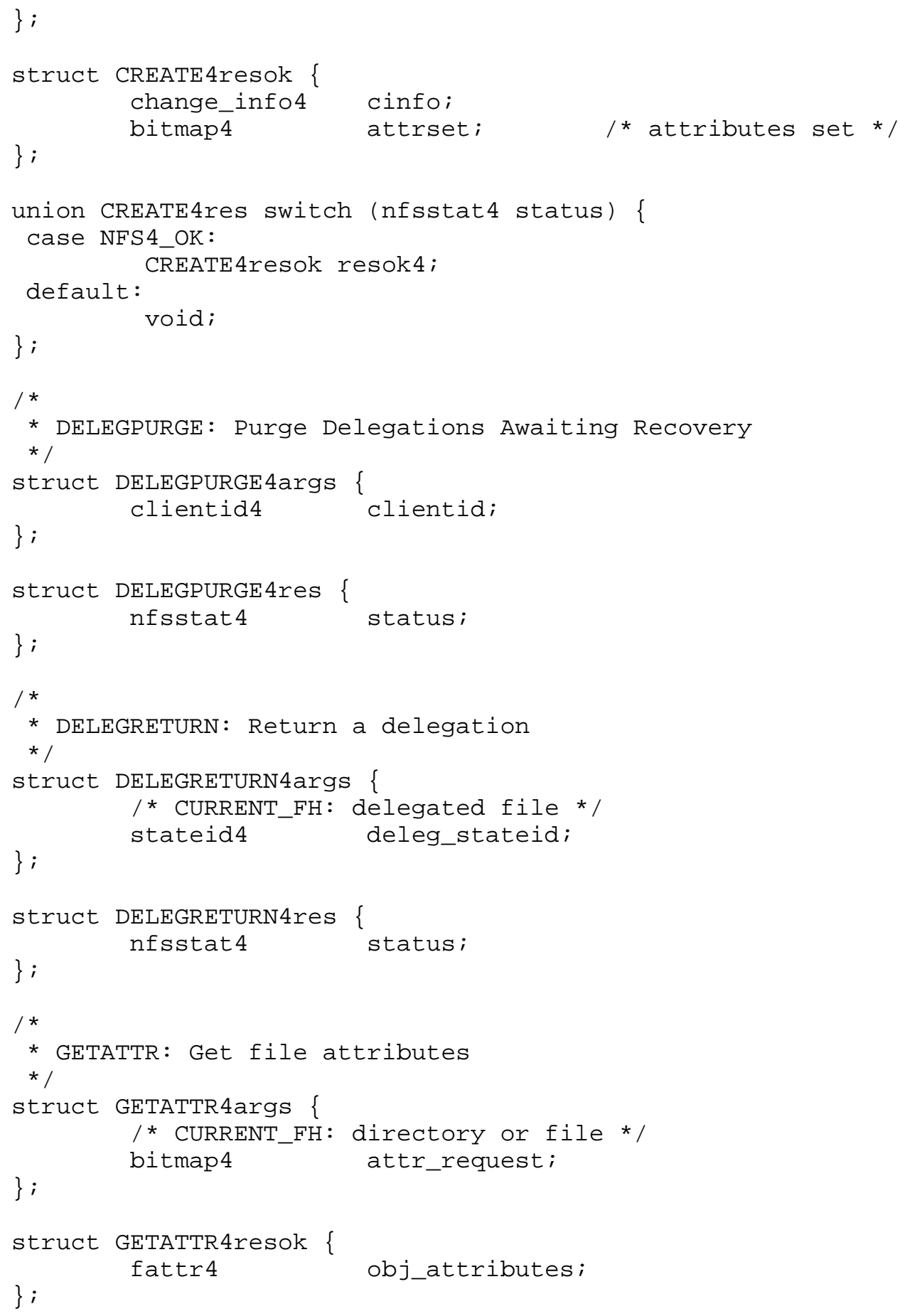




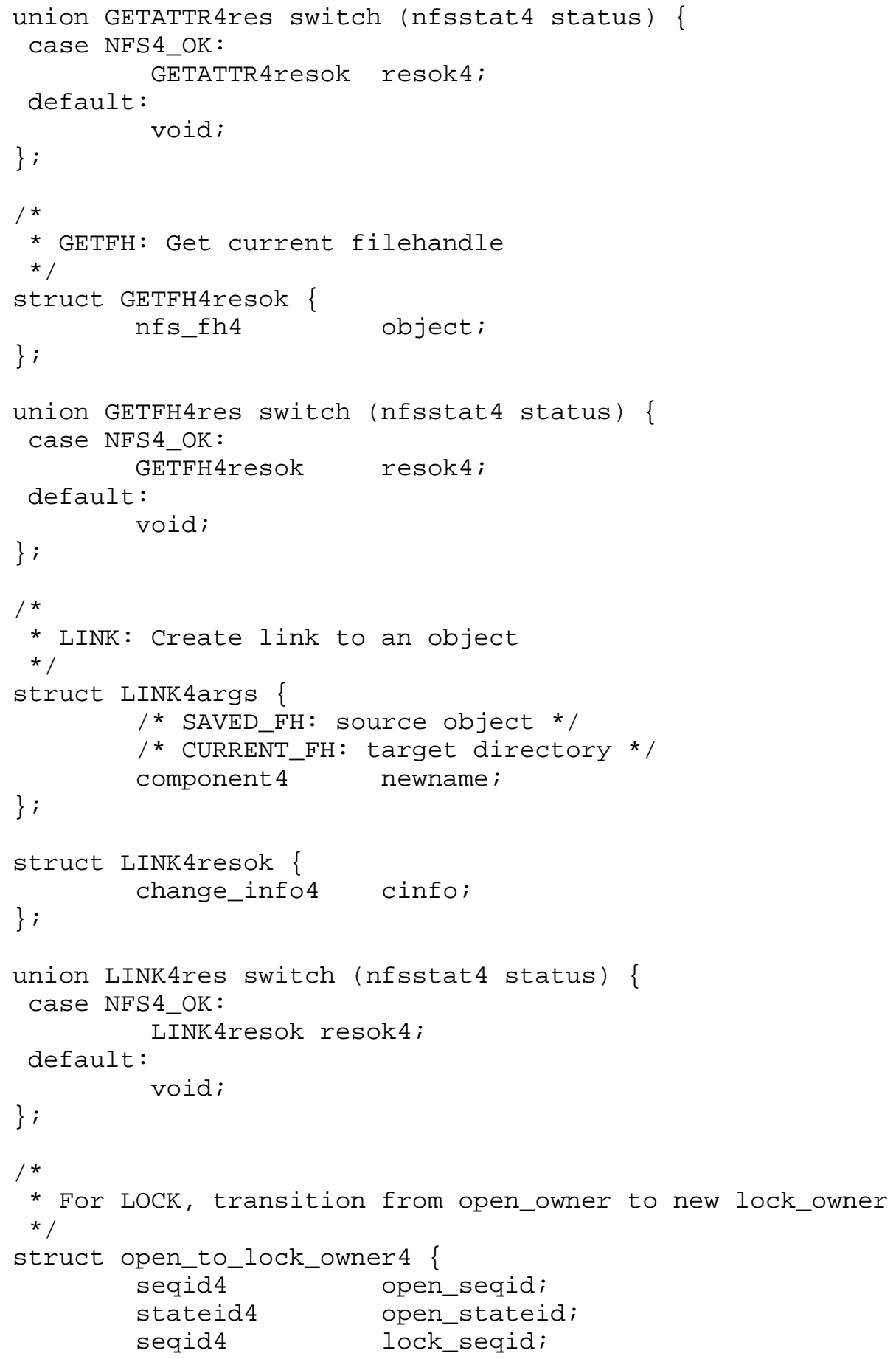




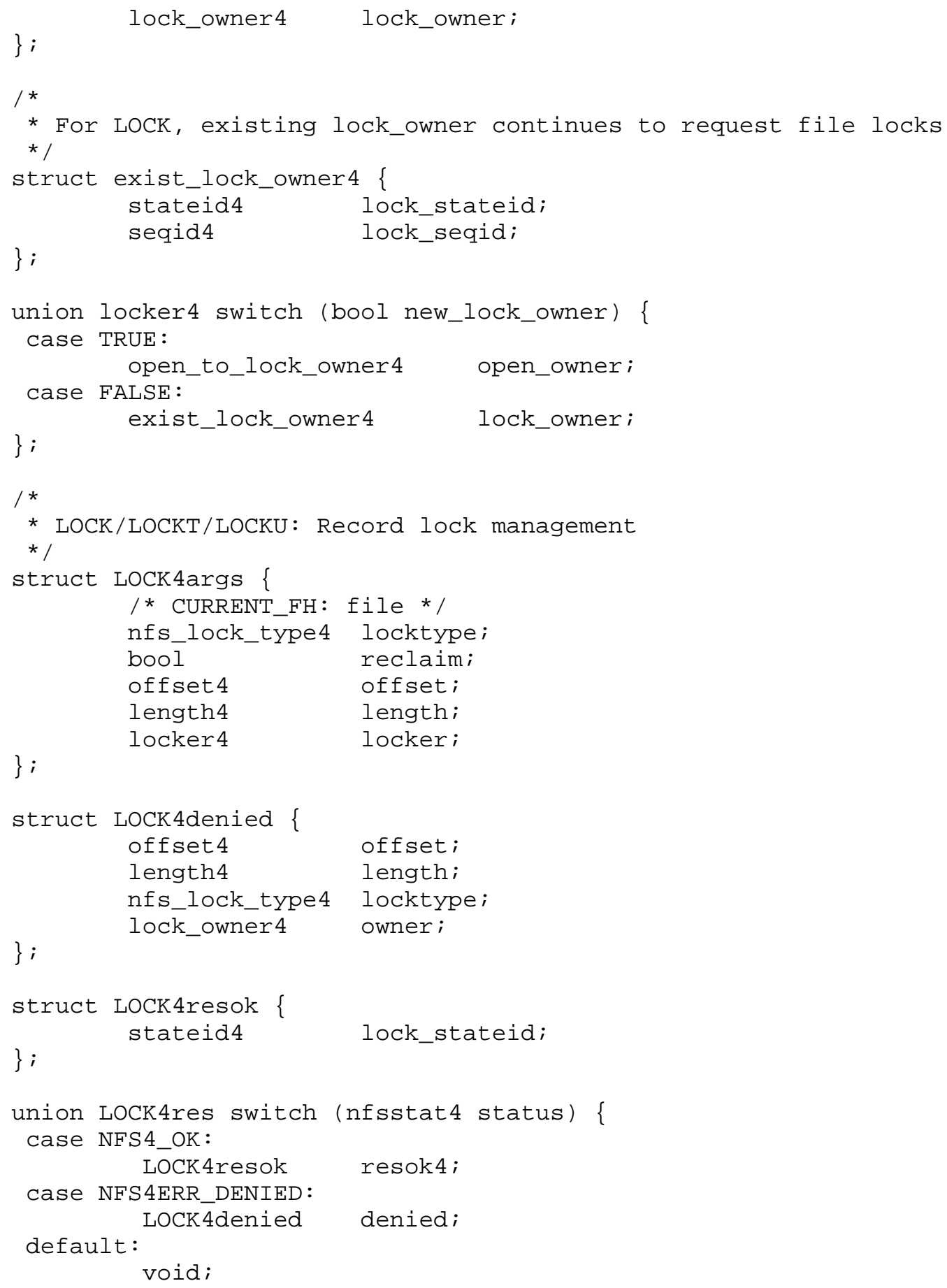


\} ;

struct LOCKT4args \{

/* CURRENT_FH: file */

nfs_lock_type 4 locktype;

offset 4 offset;

length4 length;

\} ;

lock_owner4 owner;

union LOCKT4res switch (nfsstat4 status) \{

case NFS4ERR_DENIED:

LOCK4denied denied;

case NFS4_OK:

void;

default:

void;

\} ;

struct LOCKU4args

/* CURRENT_FH: file */

nfs_lock_type 4 locktype;

seqid4 seqid;

stateid4 lock_stateid;

offset 4 offset;

\} ;

length4 length;

union LOCKU4res switch (nfsstat4 status) \{

case NFS4_OK:

default:

stateid4 lock_stateid;

\}$;$

void;

/*

* LoOKUP: Lookup filename

* 1

struct LOOKUP4args \{

/ * CURRENT_FH: directory */

\} ;

component 4 objname;

struct LOOKUP4res \{

/ * CURRENT_FH: object * /

\}

nfsstat $4 \quad$ status; 


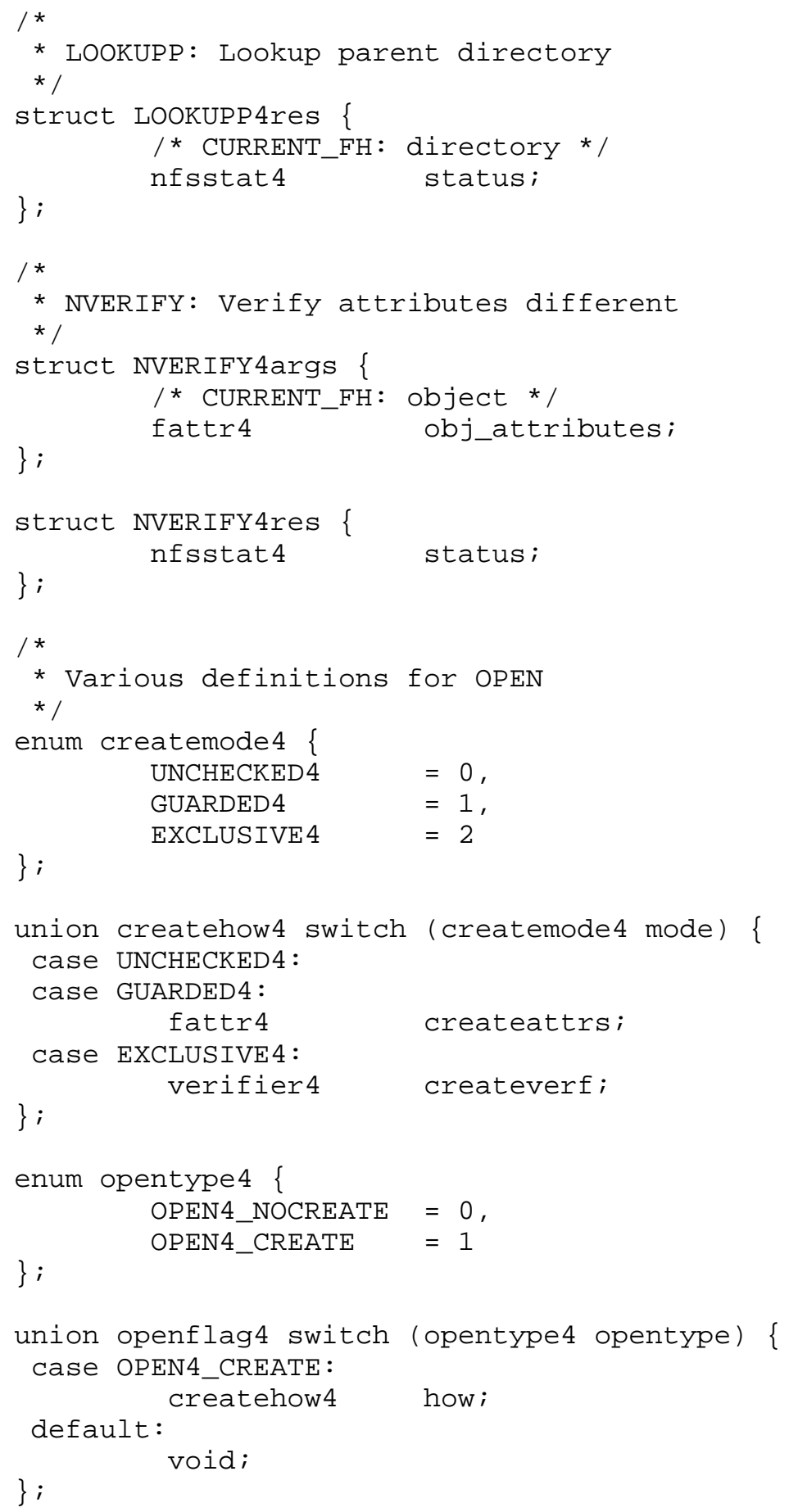




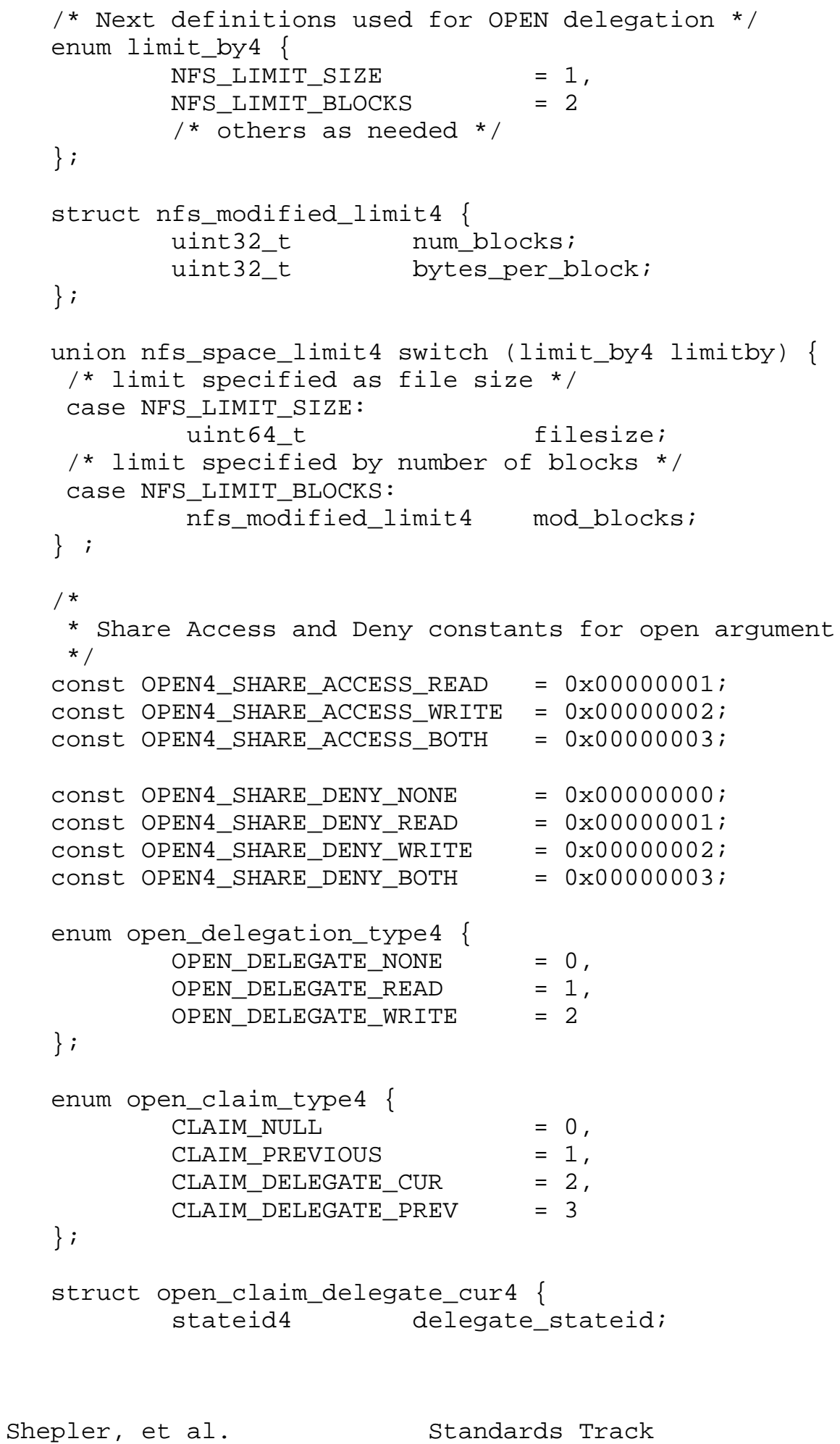




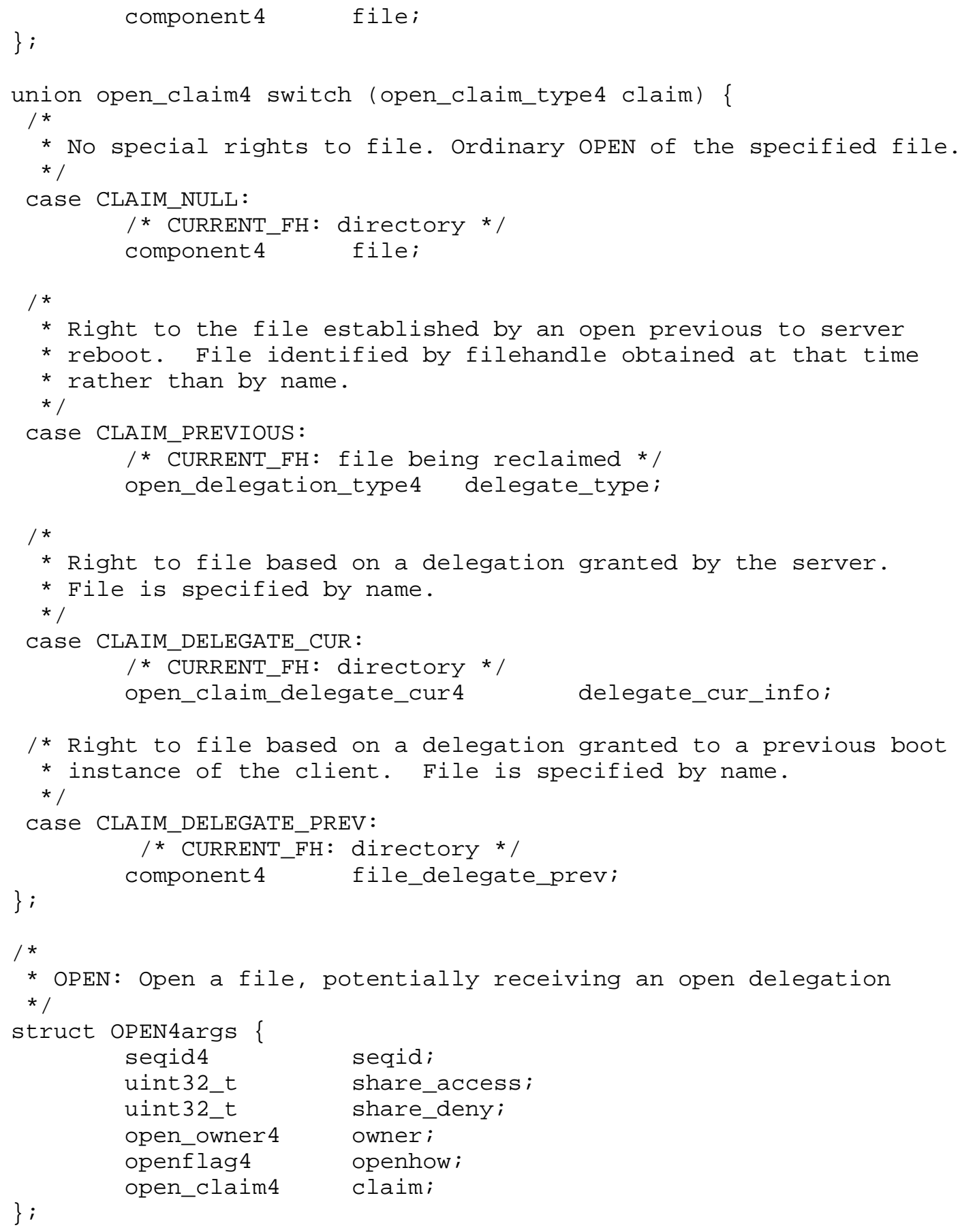




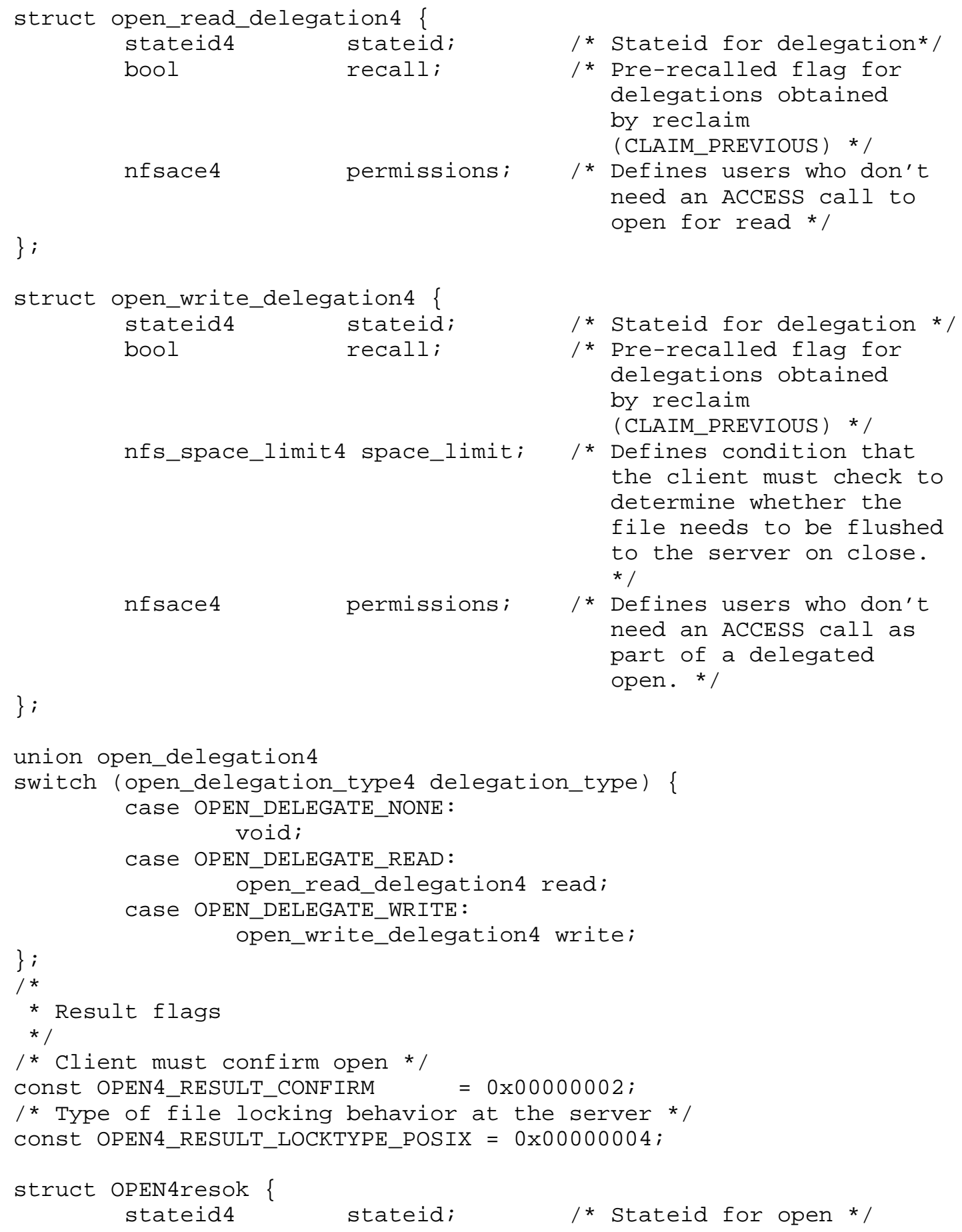




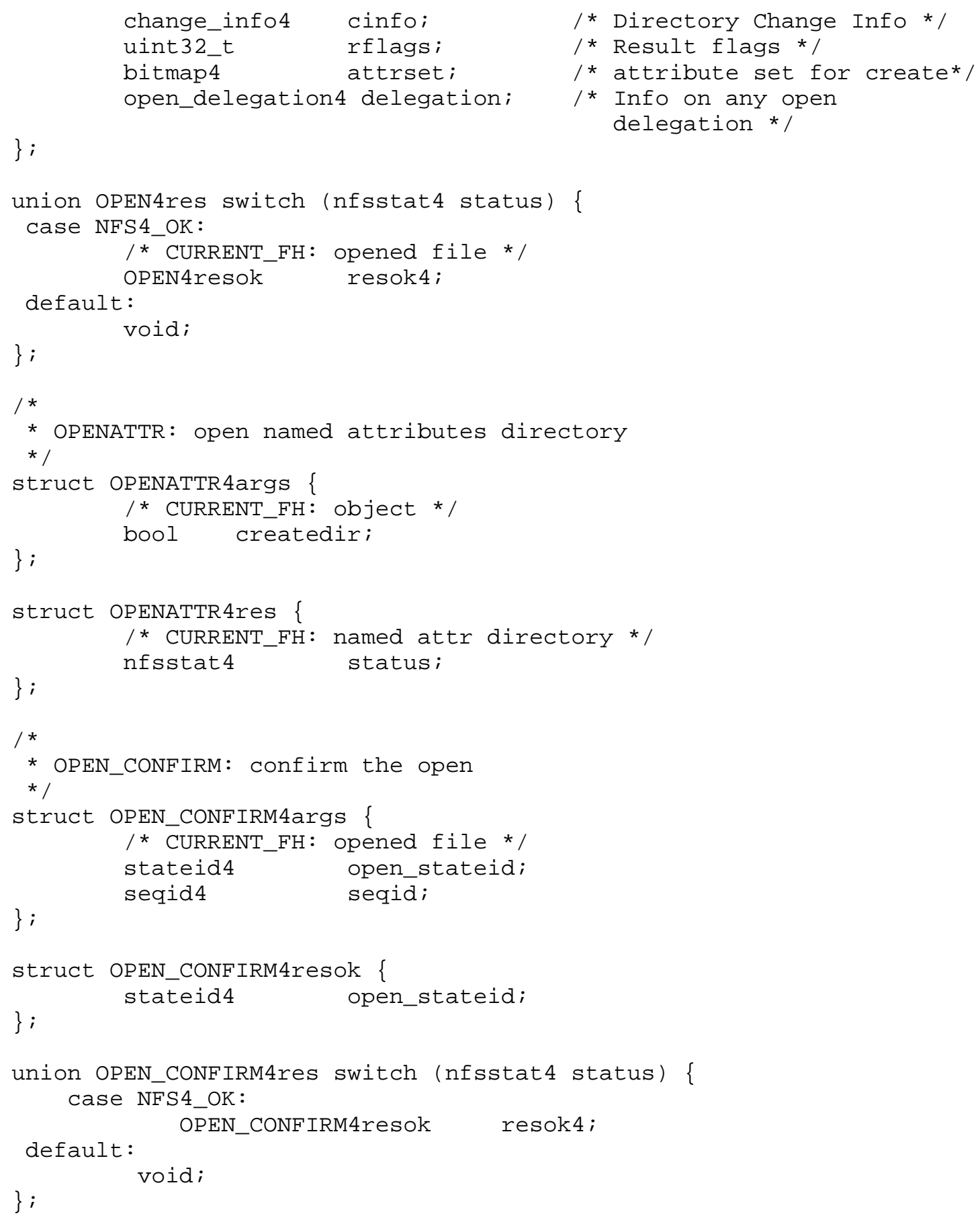




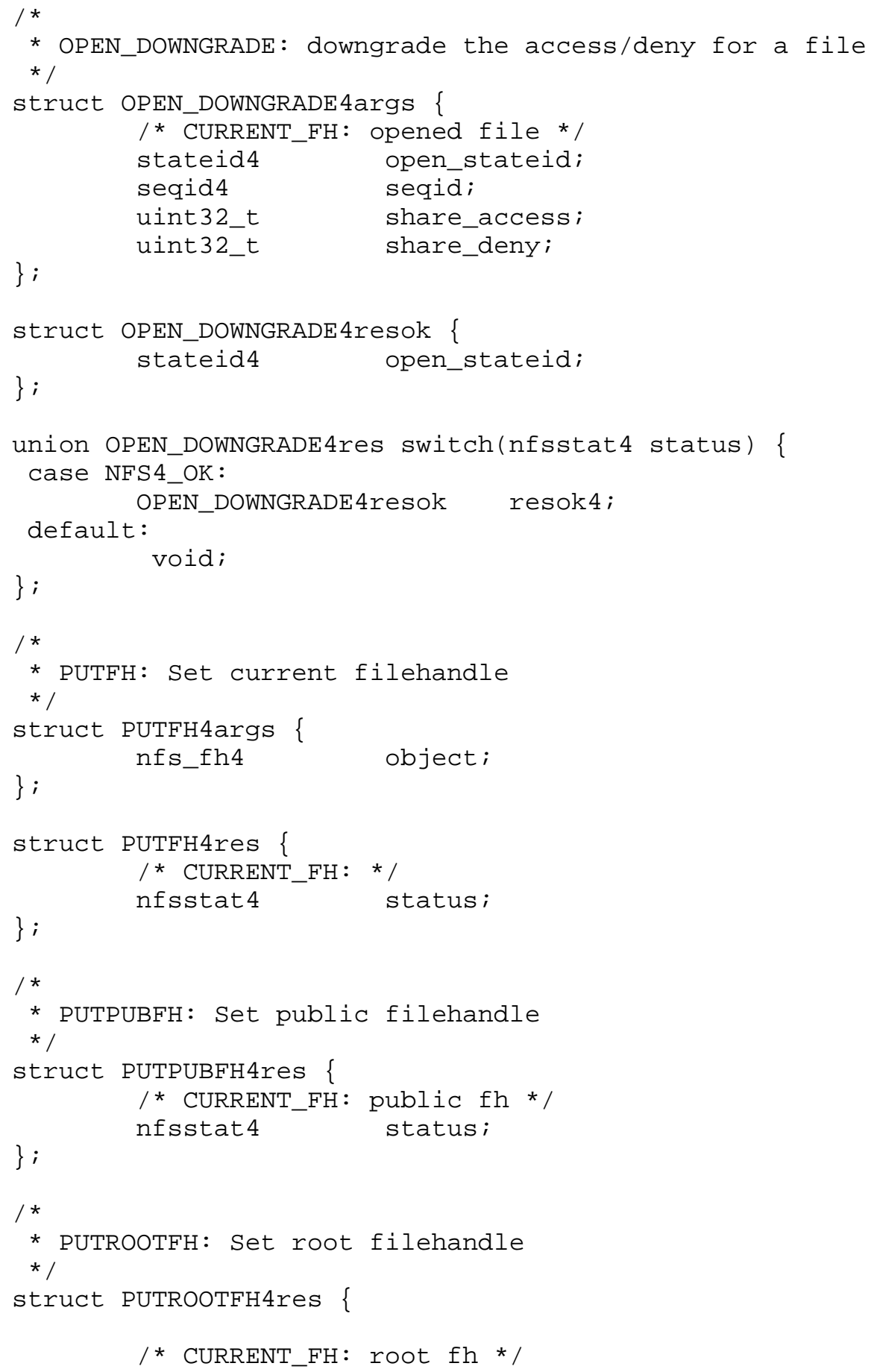




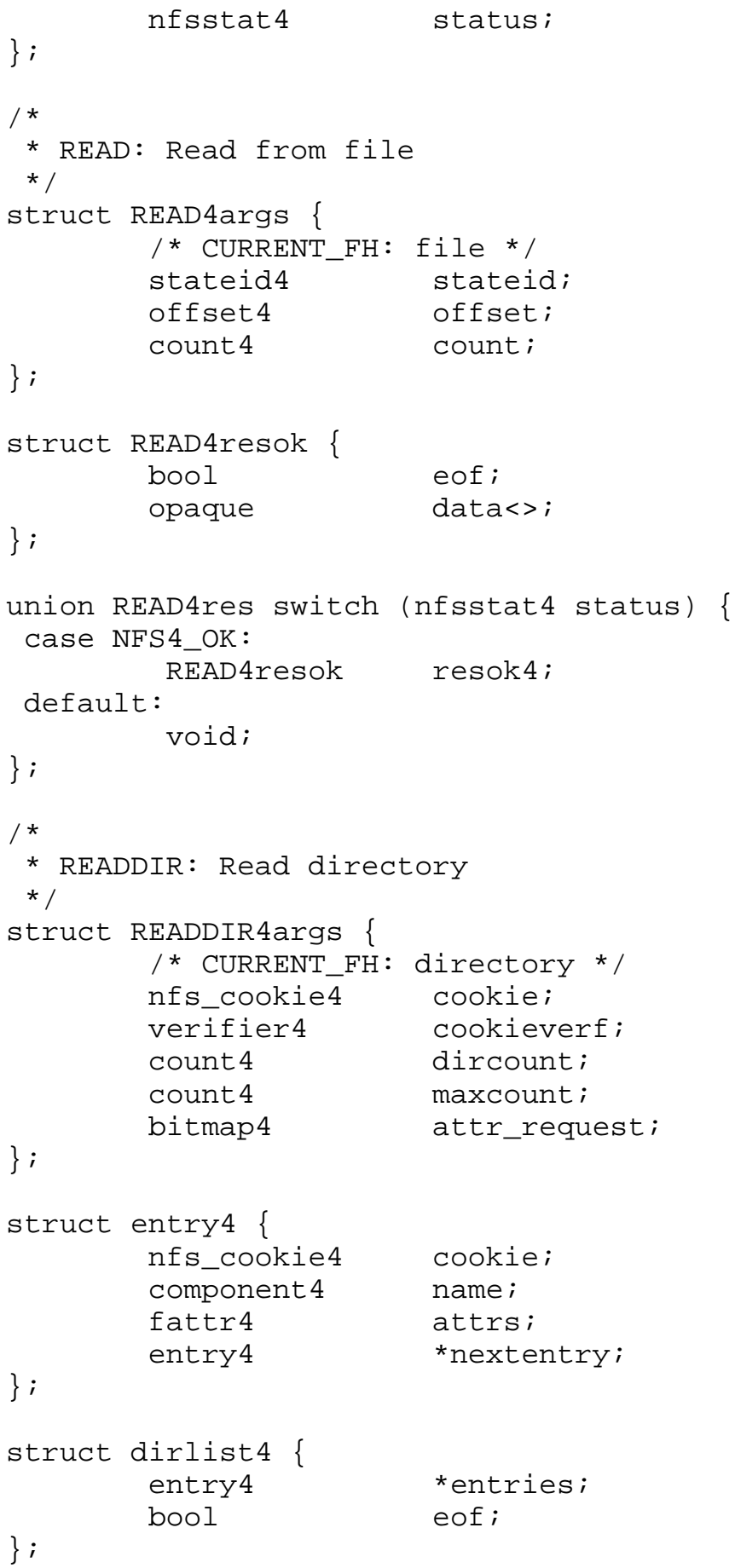




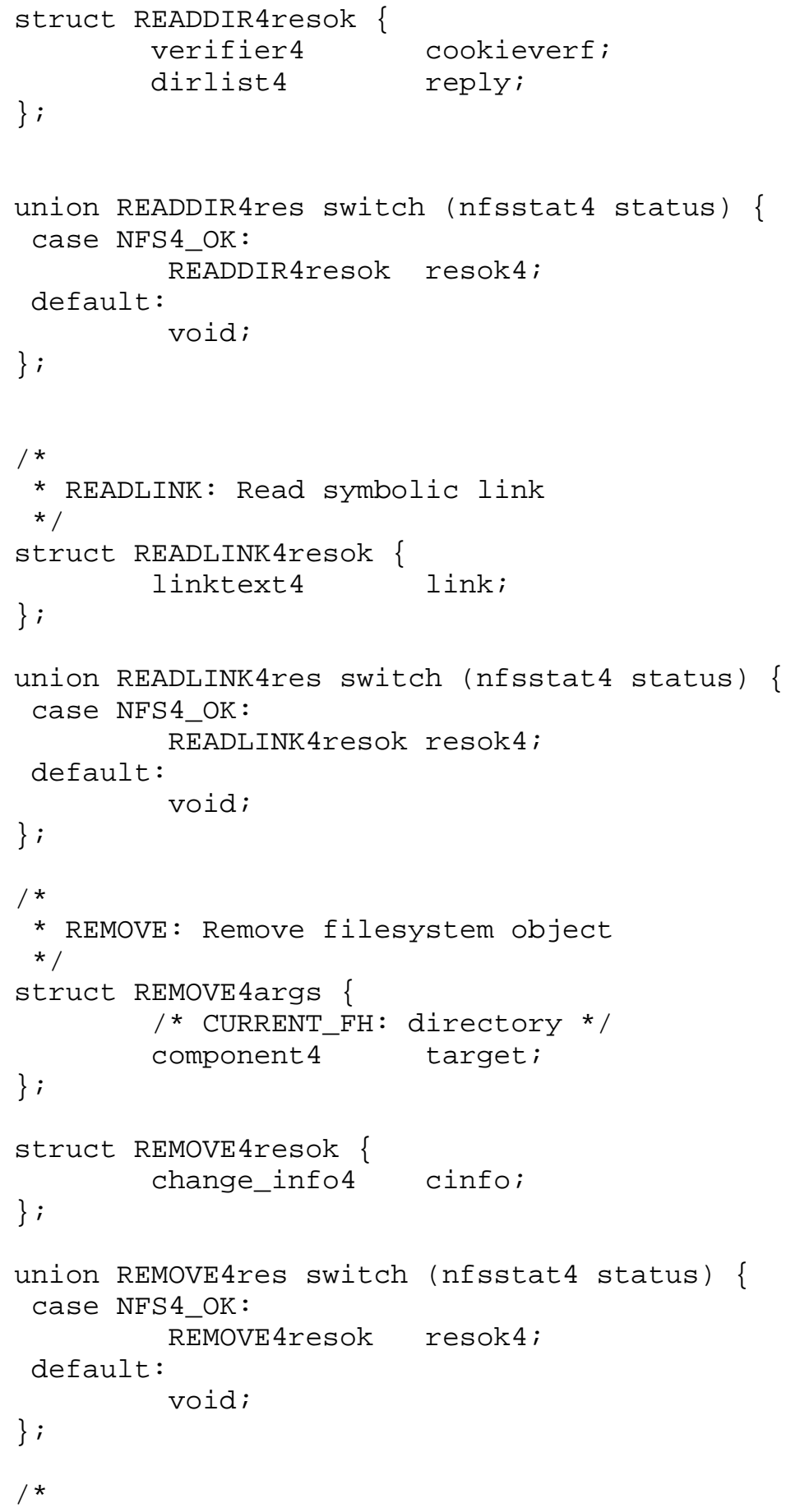


* RENAME: Rename directory entry

$\star 1$

struct RENAME4args

/* SAVED_FH: source directory */

component 4 oldname;

/ * CURRENT_FH: target directory * /

\} ;

component 4 newname;

struct RENAME4resok \{

change_info4 source_cinfo;

\} ;

change_info4 target_cinfo;

union RENAME4res switch (nfsstat4 status) \{

case NFS4_OK:

default:

RENAME 4 resok resok4;

\}$;$

void;

$/ \star$

* RENEW: Renew a Lease

* $/$

struct RENEW4args \{

clientid4 clientid;

\} ;

struct RENEW4res \{

nfsstat 4 status;

\} ;

/*

* RESTOREFH: Restore saved filehandle

*

struct RESTOREFH4res \{

/* CURRENT_FH: value of saved fh */

\} ;

nfsstat 4 status;

/*

* SAVEFH: Save current filehandle

* 1

struct SAVEFH4res \{

/* SAVED_FH: value of current fh */

nfsstat 4 status; 
\}$;$

$/ \star$

* SECINFO: Obtain Available Security Mechanisms

* $/$

struct SECINFO4args \{

/* CURRENT_FH: directory */

\}$;$

component 4 name;

$/ *$

* From RFC 2203

* /

enum rpc_gss_svc_t \{

$\begin{array}{ll}\text { RPC_GSS_SVC_NONE } & =1, \\ \text { RPC_GSS_SVC_INTEGRITY } & =2, \\ \text { RPC_GSS_SVC_PRIVACY } & =3\end{array}$

\}$;$

struct rpcsec_gss_info \{

sec_oid4 oid;

qop 4 qop;

\} ;

rpc_gss_svc_t service;

/* RPCSEC_GSS has a value of ' 6 ' - See RFC 2203 */

union secinfo4 switch (uint32_t flavor) \{

case RPCSEC_GSS:

default:

rpcsec_gss_info flavor_info;

\}

void;

typedef secinfo4 SECINFO4resok<>;

union SECINFO4res switch (nfsstat4 status) \{

case NFS4_OK:

default :

SECINFO4resok resok4;

\}$;$

void;

/*

* SETATTR: Set attributes

* /

struct SETATTR4args \{

/* CURRENT_FH: target object */ 


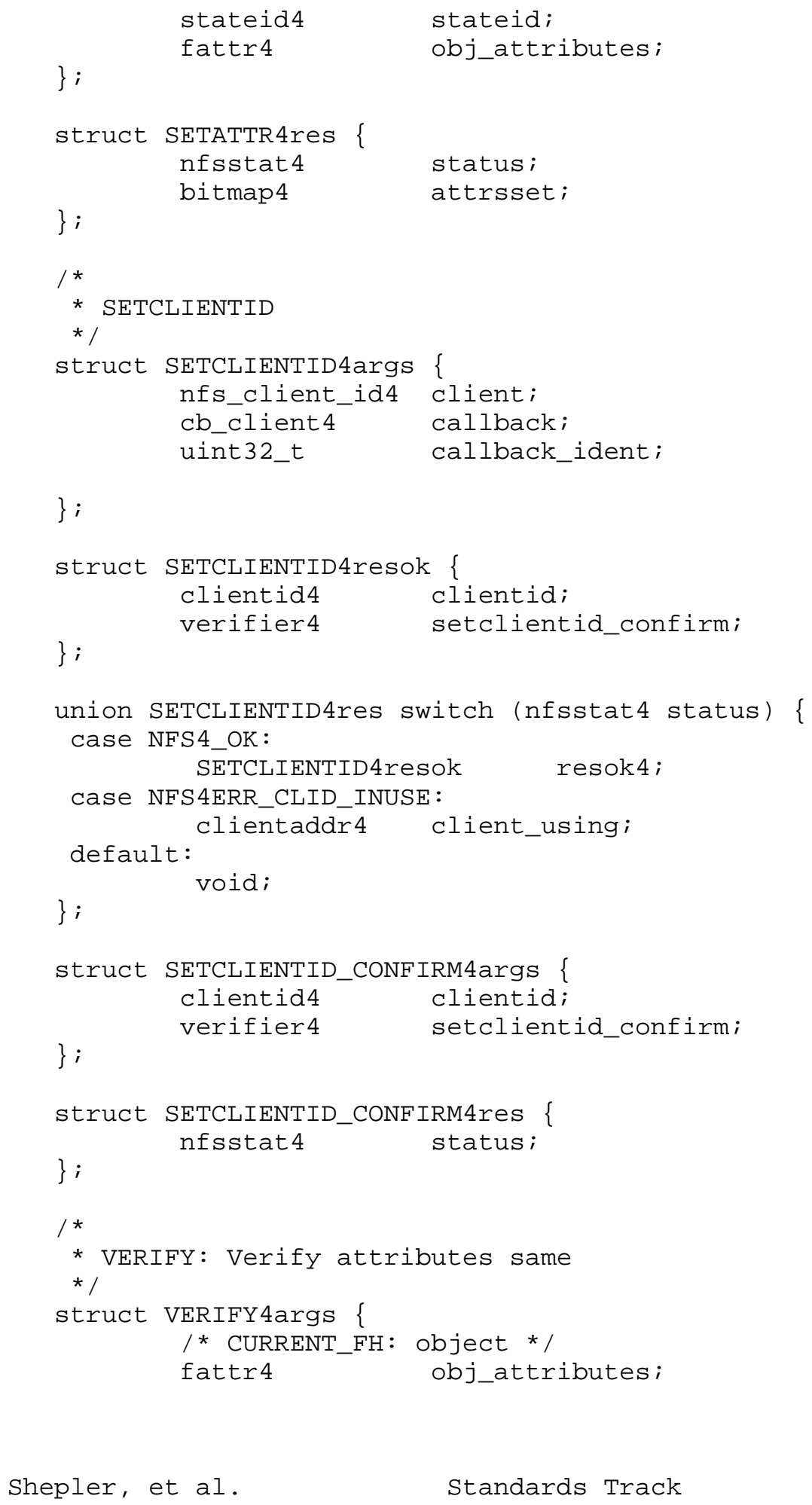


\} ;

struct VERIFY4res \{ nfsstat 4 status;

\} ;

/*

* WRITE: Write to file

$\star /$

enum stable_how 4 \{

UNSTABLE4 $=0$,

DATA_SYNC4 $=1$,

\} ;

FILE_SYNC4 $=2$

struct WRITE4args \{

/ * CURRENT_FH: file */

stateid4

offset 4

stateid;

stable_how 4

offset;

opague

\}$;$

data<>;

struct WRITE4resok \{

count 4

count;

stable_how 4

\}$;$

verifier4 writeverf.

union WRITE4res switch (nfsstat4 status) \{ case NFS4_OK:

WRITE4resok resok 4 ;

default:

void;

\}$;$

/*

* RELEASE_LOCKOWNER: Notify server to release lockowner

* 1

struct RELEASE_LOCKOWNER4args \{

lock_owner4 lock_owner;

\}$;$

struct RELEASE_LOCKOWNER4res \{

nfsstat 4 status;

\} ;

/*

Shepler, et al.

Standards Track

[Page 262] 


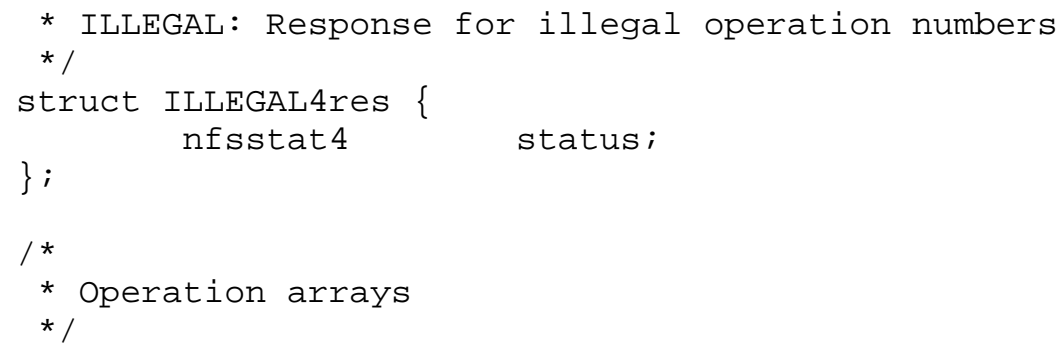




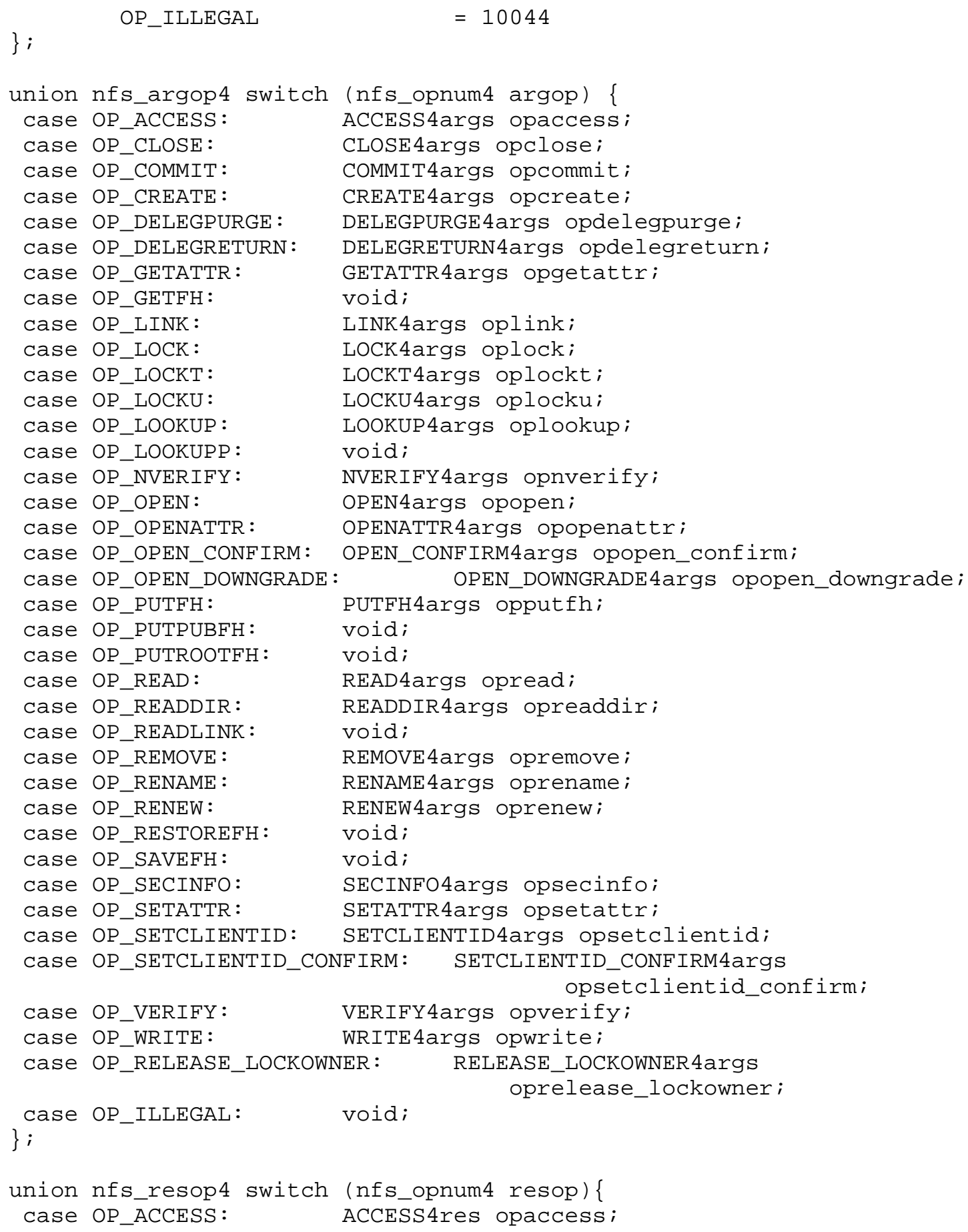




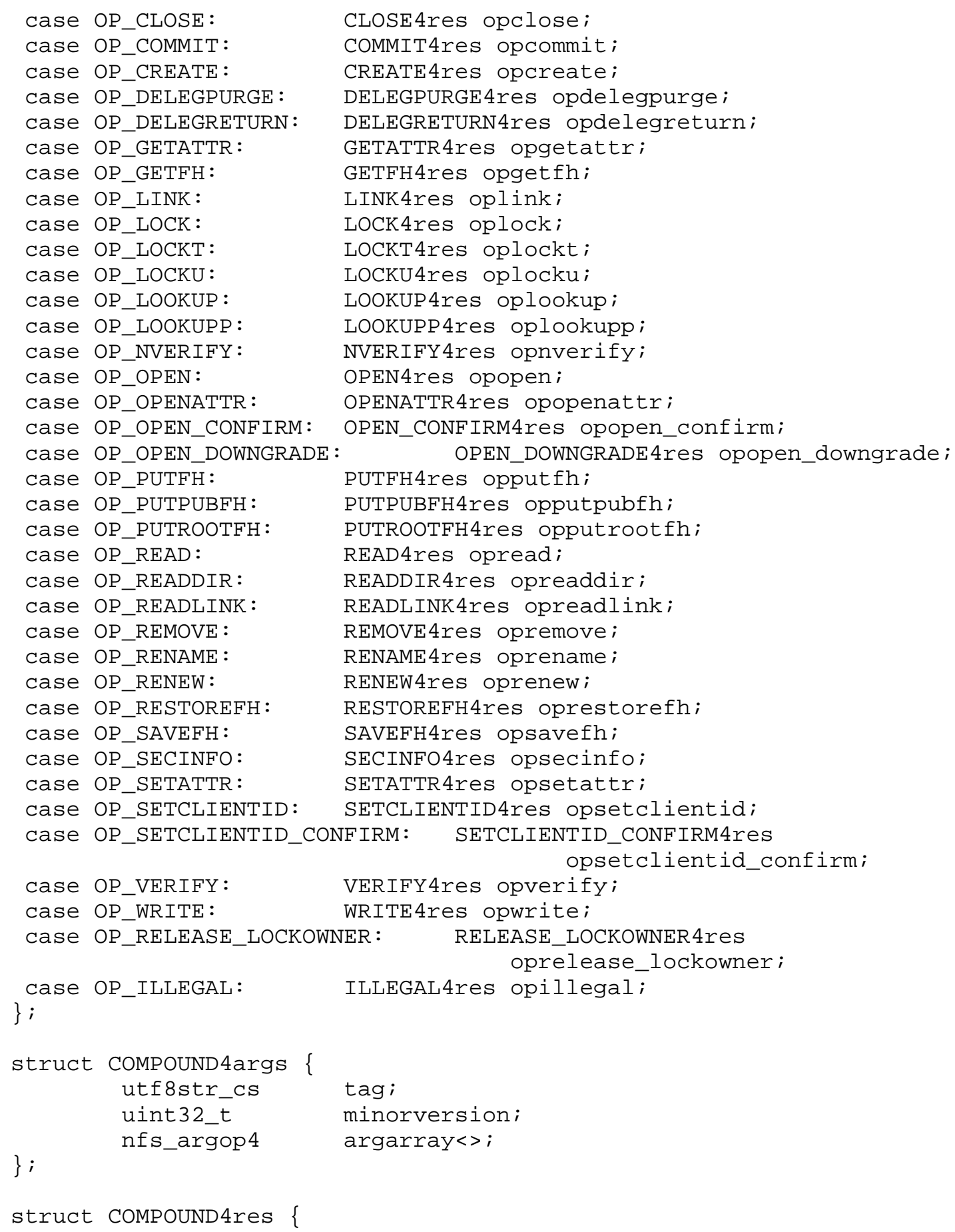




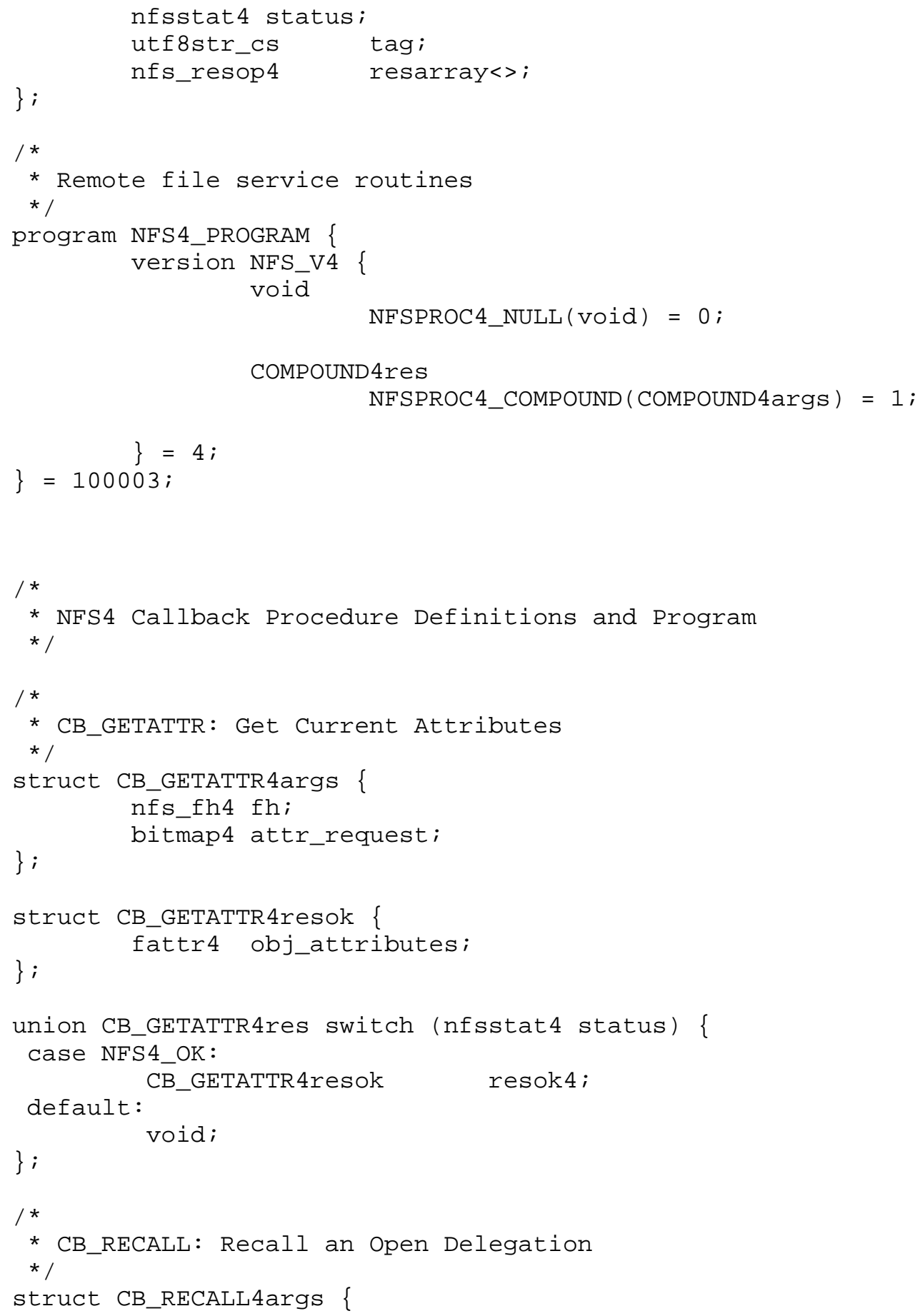




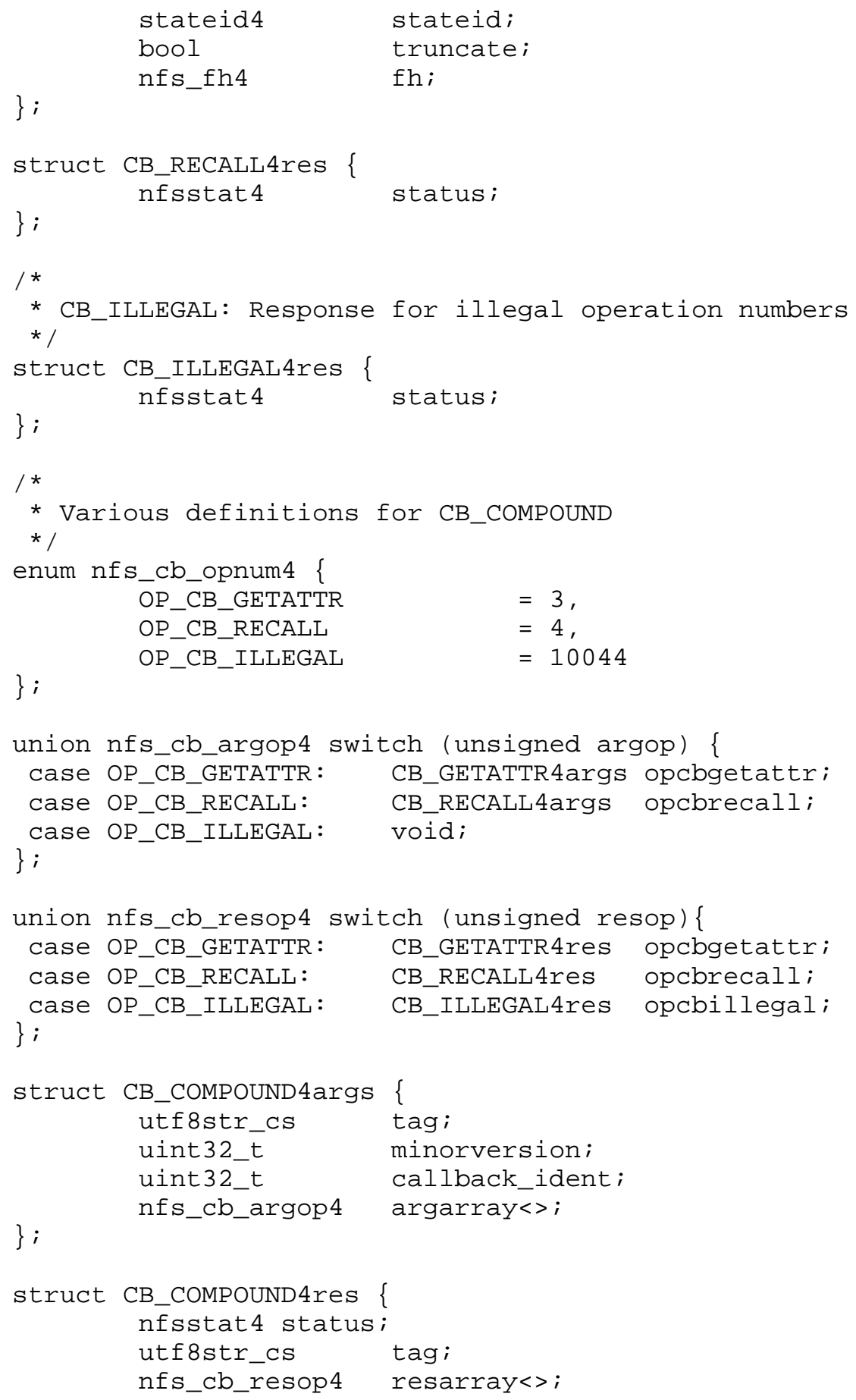


\} ;

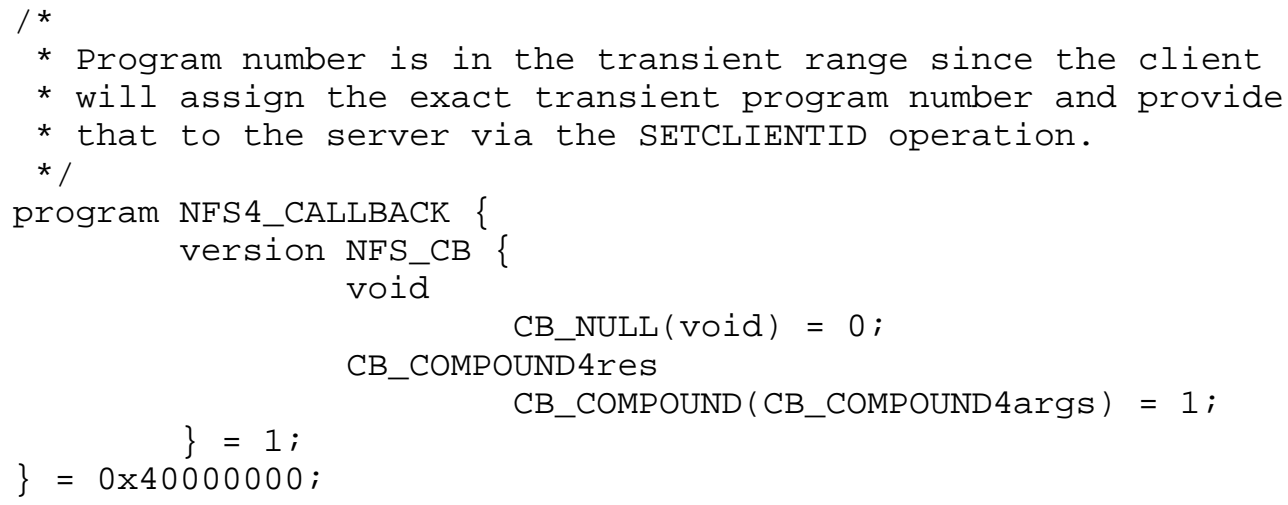

19. Acknowledgements

The authors thank and acknowledge:

Neil Brown for his extensive review and comments of various documents. Rick Macklem at the University of Guelph, Mike Frisch, Sergey Klyushin, and Dan Trufasiu of Hummingbird Ltd., and Andy Adamson, Bruce Fields, Jim Rees, and Kendrick Smith from the CITI organization at the University of Michigan, for their implementation efforts and feedback on the protocol specification. Mike Kupfer for his review of the file locking and ACL mechanisms. Alan Yoder for his input to ACL mechanisms. Peter Astrand for his close review of the protocol specification. Ran Atkinson for his constant reminder that users do matter.

20. Normative References

[ ISO10646]

[RFC793]

[RFC1831]

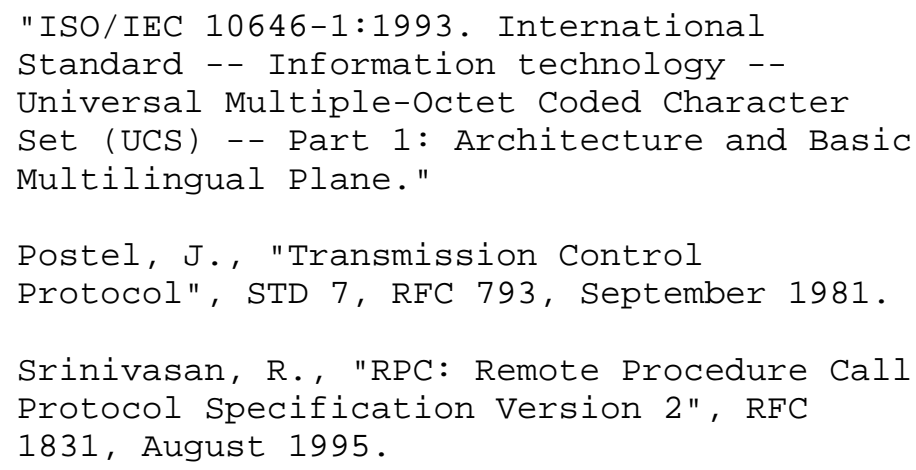

Standards Track

[Page 268] 
[RFC1832]

[RFC2373]

[RFC1964]

[RFC2025]

[RFC2119]

[RFC2203]

[RFC2277]

[RFC2279]

[RFC2623]

[RFC2743]

[RFC2847]

[RFC3010]
Srinivasan, R., "XDR: External Data Representation Standard", RFC 1832, August 1995.

Hinden, R. and S. Deering, "IP Version 6 Addressing Architecture", RFC 2373, July 1998 .

Linn, J., "The Kerberos Version 5 GSS-API Mechanism", RFC 1964, June 1996.

Adams, C., "The Simple Public-Key GSS-API Mechanism (SPKM)", RFC 2025, October 1996.

Bradner, S., "Key words for use in RFCs to Indicate Requirement Levels", BCP 14, RFC 2119, March 1997.

Eisler, M., Chiu, A. and L. Ling, "RPCSEC_GSS Protocol Specification", RFC 2203, september 1997.

Alvestrand, H., "IETF Policy on Character Sets and Languages", BCP 19, RFC 2277, January 1998.

Yergeau, F., "UTF-8, a transformation format of ISO 10646", RFC 2279, January 1998 .

Eisler, M., "NFS Version 2 and Version 3 Security Issues and the NFS Protocol's Use of RPCSEC_GSS and Kerberos V5", RFC 2623, June 1999 .

Linn, J., "Generic Security Service Application Program Interface, Version 2, Update 1", RFC 2743, January 2000.

Eisler, M., "LIPKEY - A Low Infrastructure Public Key Mechanism Using SPKM", RFC 2847, June 2000 .

Shepler, S., Callaghan, B., Robinson, D., Thurlow, R., Beame, C., Eisler, M. and D. Noveck, "NFS version 4 Protocol", RFC 3010, December 2000 . 
[RFC3454]

[Unicode1]

[Unicode2]

21. Informative References

[Floyd]

[Gray]

[Juszczak ]
Hoffman, P. and P. Blanchet, "Preparation
of Internationalized Strings
("stringprep")", RFC 3454, December 2002.

The Unicode Consortium, "The Unicode Standard, Version 3.0", Addison-Wesley Developers Press, Reading, MA, 2000. ISBN 0-201-61633-5.

More information available at: http://www.unicode.org/

"Unsupported Scripts" Unicode, Inc., The Unicode Consortium, P.O. Box 700519, San Jose, CA 95710-0519 USA, September 1999. http://www. unicode.org/unicode/standard/ unsupported.html

S. Floyd, V. Jacobson, "The Synchronization of Periodic Routing Messages," IEEE/ACM Transactions on Networking, 2(2), pp. 122136, April 1994.

C. Gray, D. Cheriton, "Leases: An Efficient Fault-Tolerant Mechanism for Distributed File Cache Consistency," Proceedings of the Twelfth Symposium on Operating Systems Principles, p. 202-210, December 1989.

Juszczak, Chet, "Improving the Performance and Correctness of an NFS Server," USENIX Conference Proceedings, USENIX Association, Berkeley, CA, June 1990, pages 53-63. Describes reply cache implementation that avoids work in the server by handling duplicate requests. More important, though listed as a side-effect, the reply cache aids in the avoidance of destructive nonidempotent operation re-application -improving correctness. 
[Kazar]

[Macklem]

[Mogul]

[Nowicki]

[Pawlowski]

[RFC1094]

[RFC1345]

Shepler, et al.
Kazar, Michael Leon, "Synchronization and Caching Issues in the Andrew File System," USENIX Conference Proceedings, USENIX Association, Berkeley, CA, Dallas Winter 1988, pages 27-36. A description of the cache consistency scheme in AFS. Contrasted with other distributed file systems .

Macklem, Rick, "Lessons Learned Tuning the 4.3BSD Reno Implementation of the NFS Protocol," Winter USENIX Conference Proceedings, USENIX Association, Berkeley, CA, January 1991. Describes performance work in tuning the 4.3BSD Reno NFS implementation. Describes performance improvement (reduced CPU loading) through elimination of data copies.

Mogul, Jeffrey C., "A Recovery Protocol for Spritely NFS," USENIX File System Workshop Proceedings, Ann Arbor, MI, USENIX Association, Berkeley, CA, May 1992. Second paper on Spritely NFS proposes a lease-based scheme for recovering state of consistency protocol.

Nowicki, Bill, "Transport Issues in the Network File system," ACM SIGCOMM newsletter Computer Communication Review, April 1989. A brief description of the basis for the dynamic retransmission work.

Pawlowski, Brian, Ron Hixon, Mark Stein, Joseph Tumminaro, "Network Computing in the UNIX and IBM Mainframe Environment," Uniforum '89 Conf. Proc., (1989) Description of an NFS server implementation for IBM's MVS operating system.

Sun Microsystems, Inc., "NFS: Network File System Protocol Specification", RFC 1094, March 1989.

Simonsen, K., "Character Mnemonics \& Character Sets", RFC 1345, June 1992.

Standards Track

[Page 271] 
[RFC1813]

[RFC3232]

[RFC1833]

[RFC2054]

[RFC2055]

[RFC2152]

[RFC2224]

[RFC2624]

[RFC2755]

[Sandberg]
Callaghan, B., Pawlowski, B. and P. Staubach, "NFS Version 3 Protocol Specification", RFC 1813, June 1995.

Reynolds, J., Editor, "Assigned Numbers: RFC 1700 is Replaced by an On-line Database", RFC 3232, January 2002.

Srinivasan, R., "Binding Protocols for ONC RPC Version 2", RFC 1833, August 1995.

Callaghan, B., "WebNFS Client Specification", RFC 2054, October 1996.

Callaghan, B., "WebNFS Server Specification", RFC 2055, October 1996.

Goldsmith, D. and M. Davis, "UTF-7 A MailSafe Transformation Format of Unicode", RFC 2152, May 1997.

Callaghan, B., "NFS URL Scheme", RFC 2224, October 1997.

Shepler, S., "NFS Version 4 Design Considerations", RFC 2624, June 1999.

Chiu, A., Eisler, M. and B. Callaghan, "Security Negotiation for WebNFS", RFC 2755, June 2000.

Sandberg, R., D. Goldberg, S. Kleiman, D. Walsh, B. Lyon, "Design and Implementation of the Sun Network Filesystem," USENIX Conference Proceedings, USENIX Association, Berkeley, CA, Summer 1985. The basic paper describing the Sunos implementation of the NFS version 2 protocol, and discusses the goals, protocol specification and tradeoffs. 
[Srinivasan]

$[\mathrm{XNFS}]$

22. Authors' Information

22.1. Editor's Address

Spencer Shepler

Sun Microsystems, Inc. 7808 Moonflower Drive

Austin, Texas 78750

Phone: +1 512-349-9376

EMail: spencer.shepler@sun.com

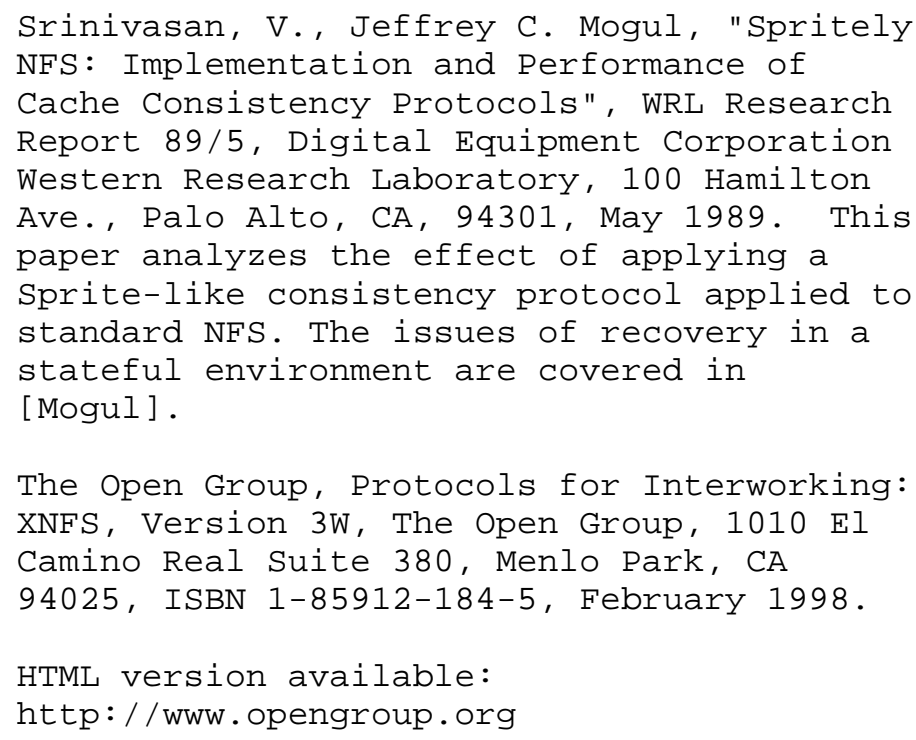




\subsection{Authors' Addresses}

Carl Beame

Hummingbird Ltd.

EMail: beame@bws.com

Brent Callaghan

Sun Microsystems, Inc.

17 Network Circle

Menlo Park, CA 94025

Phone: +1 650-786-5067

EMail: brent.callaghanesun.com

Mike Eisler

5765 Chase Point Circle

Colorado Springs, CO 80919

Phone: +1 719-599-9026

EMail: mikedeisler.com

David Noveck

Network Appliance

375 Totten Pond Road

Waltham, MA 02451

Phone: +1 781-768-5347

EMail: dnoveck@netapp.com

David Robinson

Sun Microsystems, Inc.

5300 Riata Park Court

Austin, TX 78727

Phone: +1 650-786-5088

EMail: david.robinsonesun.com

Robert Thurlow

Sun Microsystems, Inc.

500 Eldorado Blvd.

Broomfield, CO 80021

Phone: +1 650-786-5096

EMail: robert.thurlowesun.com 
23. Full Copyright statement

Copyright (C) The Internet Society (2003). All Rights Reserved.

This document and translations of it may be copied and furnished to others, and derivative works that comment on or otherwise explain it or assist in its implementation may be prepared, copied, published and distributed, in whole or in part, without restriction of any kind, provided that the above copyright notice and this paragraph are included on all such copies and derivative works. However, this document itself may not be modified in any way, such as by removing the copyright notice or references to the Internet society or other Internet organizations, except as needed for the purpose of developing Internet standards in which case the procedures for copyrights defined in the Internet Standards process must be followed, or as required to translate it into languages other than English.

The limited permissions granted above are perpetual and will not be revoked by the Internet society or its successors or assignees.

This document and the information contained herein is provided on an "AS IS" basis and THE INTERNET SOCIETY AND THE INTERNET ENGINEERING TASK FORCE DISCLAIMS ALL WARRANTIES, EXPRESS OR IMPLIED, INCLUDING BUT NOT LIMITED TO ANY WARRANTY THAT THE USE OF THE INFORMATION HEREIN WILL NOT INFRINGE ANY RIGHTS OR ANY IMPLIED WARRANTIES OF MERCHANTABILITY OR FITNESS FOR A PARTICULAR PURPOSE.

Acknowledgement

Funding for the RFC Editor function is currently provided by the Internet society. 